\title{
Sprachentwicklung vom 2. bis 8. Lebensjahr: Zur Rolle des frühen Wortschatzes
}

\author{
Dissertation \\ zur Erlangung des Doktorgrades \\ der Mathematisch-Naturwissenschaftlichen Fakultäten \\ der Georg-August-Universität zu Göttingen
}

vorgelegt von

Ann-Katrin Bockmann

aus Neumünster

Göttingen 2007 
D 7

Referent: $\quad$ Prof. Dr. Marcus Hasselhorn

Korreferent: PD Dr. Dietmar Grube

Tag der mündlichen Prüfungen: 4.7.2007 
„In uns ist der Himmel.“

Für Heidi und Muddel 


\section{Danksagung}

Mein besonderer Dank gilt meiner „kleinen“ und „großen“ Familie, die auf mich in den letzten Monaten an mancher Stelle verzichten mussten.

Stellvertretend für alle Freunde möchte ich Gregor, Jürgen und Thomas danken, die mich mit viel Humor und Unterstützung auf dem Bergpfad gehalten haben, so dass ich nicht wieder von der Talstation aufsteigen musste.

Den ASSEn - insbesondere Ariane - danke ich für die geteilte Begeisterung für das Projekt sowie die vielen Denkanstösse und Kirsten für die beständigen Erinnerungen, dass was „GESAgT“ ist, auch geschrieben werden muss.

Der „Rest“ der Abteilung Pädagogische Psychologie / Entwicklungspsychologie hat insbesondere mit dem Abo in methodischen Fragen und der guten kollegialen Stimmung sehr zum Gelingen der Arbeit beigetragen.

Professor Marcus Hasselhorn hat vielfältig mit Wissen, Erfahrung und Humor steuernd eingegriffen und Frau Prof. Kiese-Himmel danke ich für ihre kompetente Unterstützung und die ausgezeichnete „Starthilfe“.

Für die finanzielle Unterstützung sei dem Land Niedersachsen gedankt.

Und „last but not least“" möchte ich stellvertretend für alle mitwirkenden Kinder, Eltern und Einrichtungen Guschel, Katharina und Frau Dr. Waschkowski meinen herzlichen Dank für ihr großes Engagement und ihren langen Atem aussprechen (so manches Kind wird die „blauen Koffer" vermissen). 
Inhaltsverzeichnis

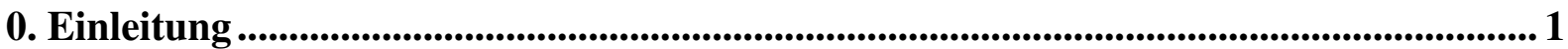



2. Methodischer Rahmen der eigenen Untersuchung ..................................................................... 9

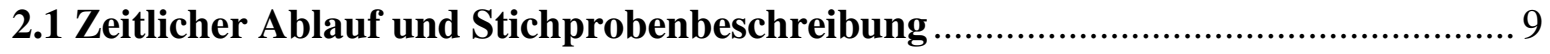

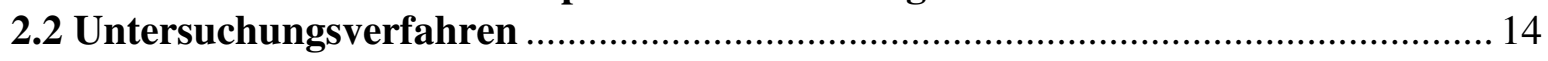

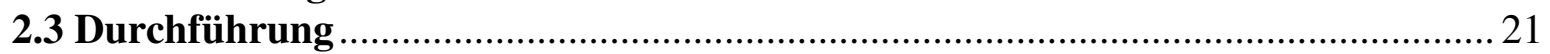

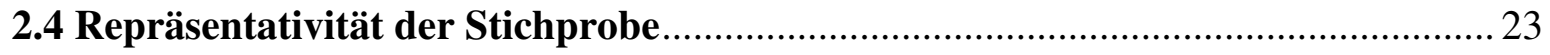

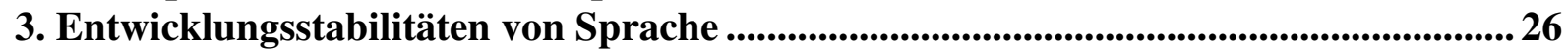

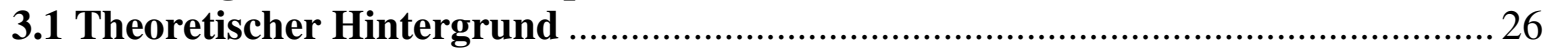

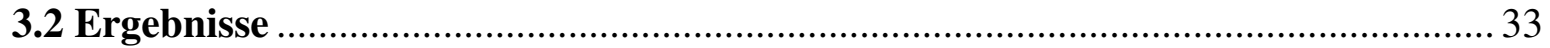

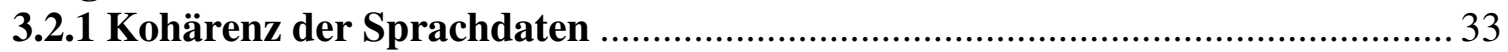

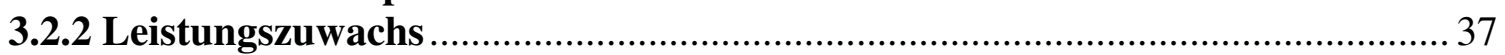

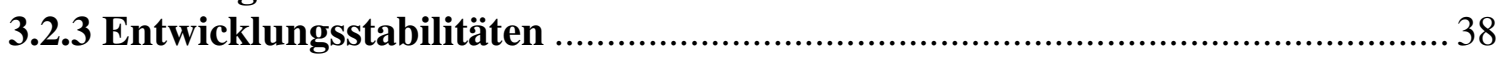

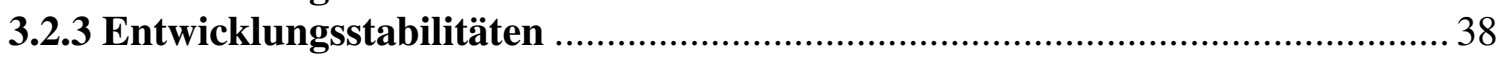



4. Der frühe Wortschatz als Prädiktor für späteres Sprachoutcome................................ 49

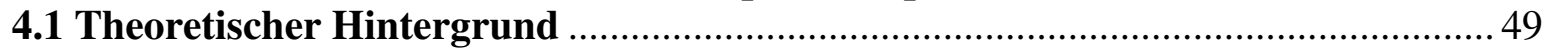





5. Frühe grammatische Fähigkeiten - Verbesserung der Vorhersage späteren

Sprachoutcomes........................................................................................................ 71

5.1 Theoretischer Hintergrund ……........................................................ 71



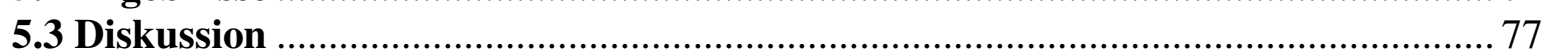

6. Sprechbeginn - Zusammenhänge zu späteren Sprachleistungen ................................... 80

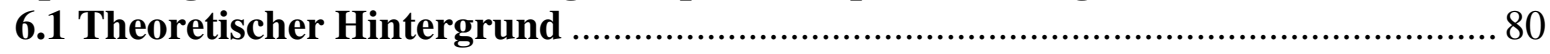

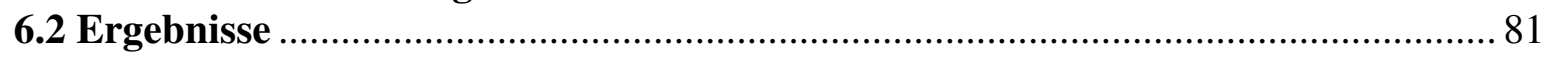

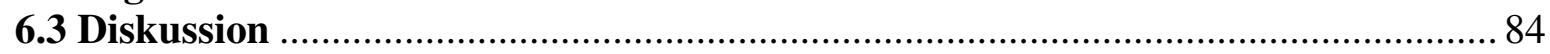

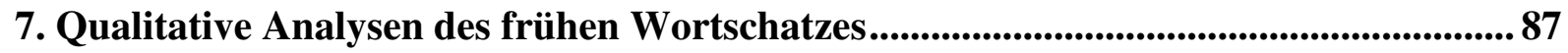

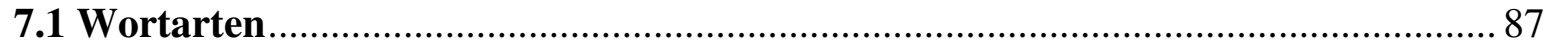





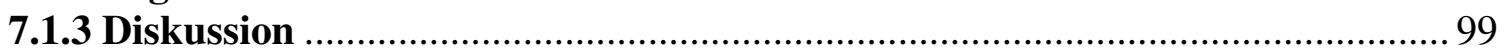

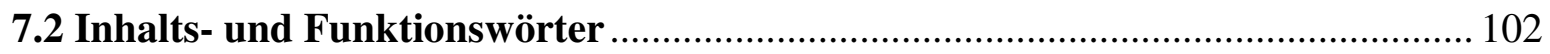

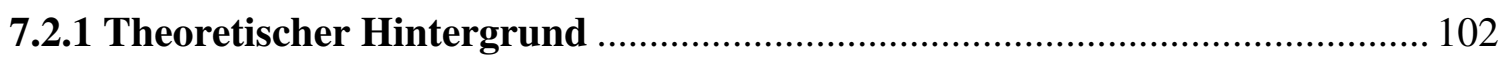

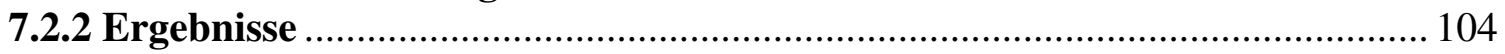

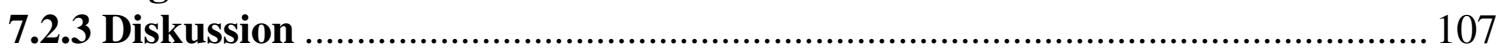

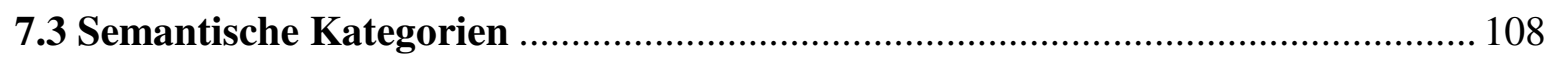



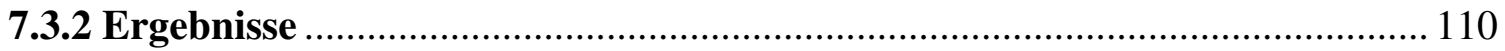

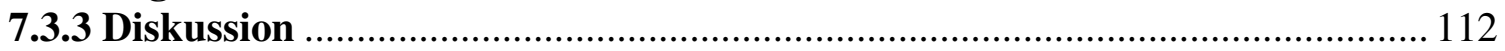

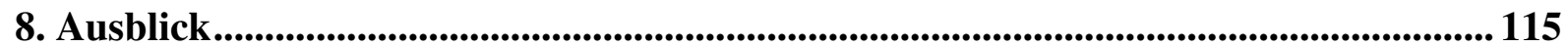

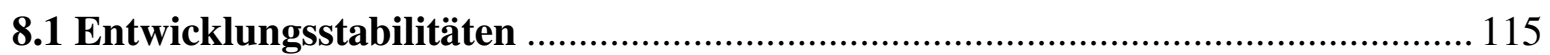

8.2 Prädiktive Validität des frühen Wortschatzes................................................. 116

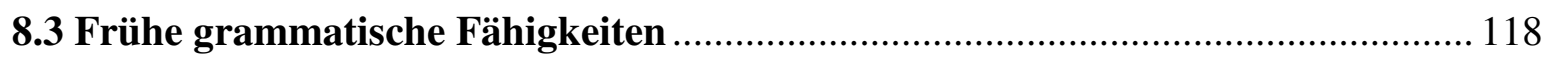



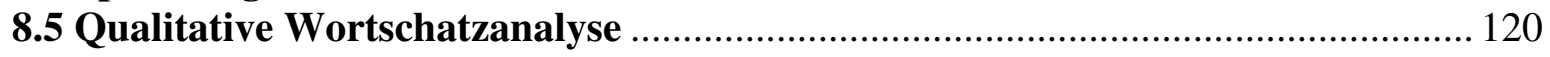

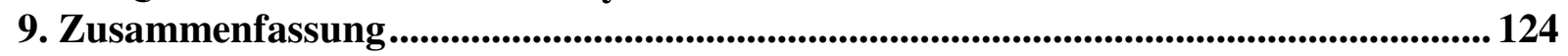






11. Tabellenverzeichnis ...................................................................................................................... 140

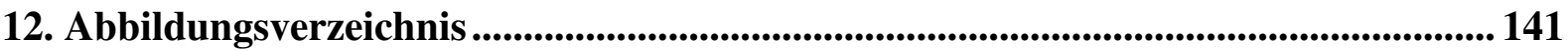

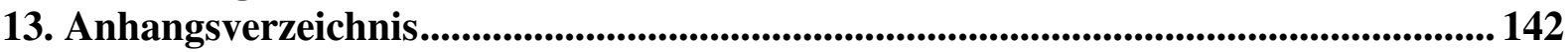

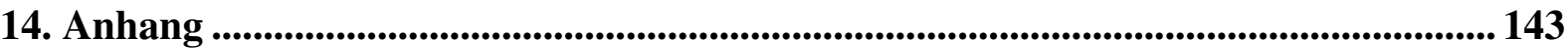




\section{Einleitung}

Um die Faszination der Sprache lassen sich viele Worte machen.

„1769 gewann Herder den Wettbewerb der Preußischen Akademie der Wissenschaften mit einem Essay, in dem er feurig die Ansicht vertrat, die Sprache sei entgegen traditioneller Annahme kein göttliches Geschenk an den Menschen, sondern dessen eigenes Werk“ (Zimmer, 1988, S. 7-8). Mit diesem Essay eröffnete Herder den Weg zu einer rationalen Erklärung der Entstehung von Sprache. Noch 200 Jahre später bezeichnete der bekannte Spracherwerbsforscher Braine (1963) den Spracherwerb als größtes und fesselndstes Mysterium der Psychologie. Grimm und Weinert (2005) stimmen der Gültigkeit dieses Satzes für die heutige Zeit zu, wenn auch die Versuche, dem Mysterium näher zu kommen, „vielfältiger und vielversprechender geworden sind“ (S. 520).

Insbesondere die frühe Sprachentwicklung stellt das Fenster für die weiteren Lern- und Entwicklungsoptionen eines Kindes dar, und der frühe Wortschatz konnte sich als Prädiktor für die spätere Sprachentwicklung etablieren (Toppelberg \& Shapiro, 2000).

Motivation für vorliegende Arbeit war, zu prüfen, wie gut sich die sprachliche Entwicklung aus den frühen Wortschatzdaten auch über eine große Zeitspanne und für eine Stichprobe normal entwickelter deutschsprachiger Kinder vorhersagen lässt, und wie stabil Sprache als Entwicklungsmerkmal über eine große Zeitspanne von bis zu 6 Jahren ist. Gilt zum Beispiel der im klinischen Alltag häufig verwendete Satz, dass aus einem „Ackergaul kein Rennpferd zu machen sei“ oder ist Sprache für eine große Zeitspanne ein Merkmal von so niedriger Stabilität, dass sehr wohl der Ackergaul von damals „wie von selbst“ zum Rennpferd von heute wird?

Einen neuen Forschungsbeitrag liefert die vorliegende Studie mit der Bearbeitung der Fragen, ob sich die Vorhersagen späteren Sprachoutcomes aus den quantitativ betrachteten Wortschatzdaten durch frühe grammatische Fähigkeiten und qualitative Wortschatzanalysen verbessern lassen und ob qualitative Wortschatzaspekte in höherem Maße mit späterem Sprachoutcome zusammenhängen als der expressive Wortschatzumfang.

Der Arbeit sind zudem Ergebnisse zur Relevanz des Alters, in dem erste Worte geäußert werden, für den weiteren Sprachentwicklungsverlauf zu entnehmen.

Alle Ergebnisse werden mit Hinblick auf ihre theoretische und praktische Relevanz diskutiert und im Ausblick wird eine Vielfalt sich ergebender weiterer potenzieller Forschungsperspektiven aufgezeigt, um dem Mysterium Sprache wieder einen Schritt näher zu kommen. 


\section{Sprachentwicklung und früher Wortschatz}

Der Erwerb von Sprache ist eine der zentralen Entwicklungsaufgaben, die ein Kind in den ersten Lebensjahren bewältigen muss und als solche ein Untersuchungsgegenstand der Entwicklungspsychologie, der seit langer Zeit entsprechend intensiv erforscht wird. Sprachliche Fähigkeiten stehen in enger Beziehung zu kognitiven und sozialen Fähigkeiten sowie zur Verhaltensregulation (Grimm, 1999). Abweichungen bzw. Störungen in der Sprachentwicklung weisen vielfach belegte Zusammenhänge mit Lern- und Schulproblemen (z.B. schlechteren Leseleistungen), Verhaltensstörungen und psychiatrischen Störungen, erhöhter Kriminalität, Intelligenzschwäche sowie Problemen im sozial-kommunikativen Bereich und der Persönlichkeitsentwicklung auf (Cheng, 2000; Rescorla, 1997; Ward, 1999).

Seine aktuelle Bedeutung erfährt der Bereich Sprache insbesondere durch Forschungen zur gestörten Sprachentwicklung, denn Verzögerungen und Störungen der Sprachentwicklung haben sich $\mathrm{zu}$ einem der Hauptgesundheitsprobleme im Kindesalter entwickelt (Paul \& Kellogg, 1997; Rescorla, 1989), was sich nicht zuletzt in der steigenden Aufmerksamkeit der Gesellschaft für diesen Problemkomplex widerspiegelt.

Prävalenzen zwischen 5 und $10 \%$ werden für Sprachentwicklungsstörungen berichtet (Law, Boyle, Harris, Harkness \& Nye, 1998), wobei unterschiedliche Angaben z. T. durch die uneinheitlichen Definitionen von Sprachentwicklungsstörung bzw. -verzögerung bedingt sind und von einer großen Anzahl unentdeckter Fälle auszugehen ist (Tomblin, Records, Buckwalter, Zhang, Smith \& O`Brien, 1997).

Darüber hinaus hat sich die Beherrschung der deutschen Sprache im Rahmen der PISA Studien als eine der wesentlichsten Schlüsselkompetenzen für Bildung herausgestellt (Deutsches Jugendinstitut [DJI], 2004) und folglich werden gerade für das Kindergartenalter Untersuchungen zur Sprachentwicklung mit Hinblick auf spätere Schulleistungen durchgeführt bzw. die Folgen früher Sprachentwicklungsabweichungen für das Schulalter untersucht.

Das Interesse an der frühen Sprachentwicklung (bis 3 Jahren) ist relativ neu (Rescorla, 1997). Gerade die frühe Sprachentwicklung ist nach Grimm, Doil, Müller und Wilde (1996) das Fenster für die allgemeinen Entwicklungsmöglichkeiten eines Kindes. Hierbei ist der Spracherwerb von Kindern, die amerikanisches oder britisches Englisch erwerben, wesentlich genauer und breiter untersucht, als der Spracherwerb deutschsprachiger Kinder (Szagun, 1996).

Betrachtet man nur Abweichungen in der frühen Sprachentwicklung (bis 3 Jahre), so lassen sich bereits in dieser frühen Phase Zusammenhänge zu späteren persistierenden 
Sprachstörungen, erhöhter Kriminalität, Lern- und Schulproblemen, späteren Sprach- und Leseleistungen sowie zu sozialen und Verhaltensproblemen feststellen (Dale, 1991; Ward, 1999).

Von besonderem Interesse ist folglich die Stabilität von Sprachentwicklung; denn sollte Sprache ein Entwicklungsmerkmal sein, welches über ausgeprägte Stabilität schon im frühen Alter und über große Zeitspannen verfügt, ließen sich aus den frühen Sprachmaßen spätere Sprachleistungen gut vorhersagen sowie die Notwendigkeit früher Interventionsmaßnahmen im Sinne der Prävention ableiten. Dieses wäre sowohl für die gestörte wie auch die normale Sprachentwicklung gültig und ein Forschungsgegenstand von hoher praktischer Bedeutung.

Zudem ist zu erwarten, dass Aussagen zur Stabilität von Sprache Rückschlüsse auf die allgemeinen Funktionsmechanismen und theoretischen Erklärungsmodelle von Sprachentwicklung ermöglichen, was Untersuchungen zur Entwicklungsstabilität von Sprache ebenfalls eine hohe grundlagentheoretische Bedeutung verleiht.

Die Stabilität von Sprache ist vielfältig untersucht für den Aspekt der Stabilität von Störungen der Sprachentwicklung (Prathanee, Thinkhamrop \& Dechongkit, 2006; Westerlund, Berglund \& Eriksson, 2006). Auch lässt sich eine Reihe von Studien zur Stabilität von Sprachentwicklung über kurze Zeitspannen finden. Bei den Studien zur Stabilität von Sprache überwiegen Untersuchungen aus dem englischen Sprachraum, die allgemein die Stabilität von Sprache nicht jedoch die Stabilität unterschiedlicher Aspekte von Sprache erfassen.

Wie sieht es jedoch mit der Stabilität von unterschiedlichen Sprachaspekten über eine große Zeitspanne (von der frühen Kindheit bis ins Grundschulalter) für eine normal entwickelte Stichprobe deutschsprachiger Kinder aus? Dieser Forschungsfrage soll in der vorliegenden Arbeit nachgegangen werden.

Sprache besteht aus unterschiedlichen Komponenten wie z.B. der Phonologie, der Morphologie, der Syntax und dem Lexikon. Diese Komponenten sind stets assoziiert und müssen parallel verarbeitet werden, können jedoch als eigenständige Wissenssysteme betrachtet werden (Grimm, 1995b). Im Laufe der Sprachentwicklung muss ein Kind die Regeln jedes einzelnen Systems sowie die Regeln ihres Zusammenspiels erlernen. Dem Lexikon bzw. Wortschatz kommt eine besondere Bedeutung innerhalb der Sprachentwicklung sowie bezogen auf die allgemeine Entwicklung des Kindes zu. Pruden, Hirsh-Pasek, Golinkoff und Hennon (2006) drücken dieses wie folgt aus: „Words are the building blocks of language. They are the social and mental currency through which we represent our world and 
communicate with others" (S. 266). Wörter sind die zentralen Bausteine der Sprache - quasi das Rohmaterial, aus denen das Kind Sätze baut und an dem es seine morphologische Entwicklung vollzieht (Grimm, 2000). Der Wortschatz als ein Teil des gesamten Lernprozesses, den Kinder in den ersten Jahren vollziehen, darf nicht unterschätzt werden. „Wortschatzentwicklung ist eine notwendige, aber nicht hinreichende Bedingung für Sprachkompetenz und -performanz sowie für die Fähigkeit, adäquat sprachlich zu kommunizieren“ (Kiese \& Kozielski, 1996, S. 6).

Die Fähigkeit, sich sprachlich auszudrücken, ist die Basis, auf dem unser Schulsystem aufbaut. Der Wortschatz ist somit ein Instrument zum Erwerb weiterer Leistungen. Die sprachliche Kompetenz, und hier vor allem der Wortschatz, gilt als Maßstab für Intelligenz, und in vielen Schulleistungstests und verbalen Intelligenztests ist ein Wortschatztest als Subtest enthalten (z.B. in der Kaufmann-Assessment Battery for Children [Melchers \& Preuß, 1991]). Dieses verdeutlichen Melchers und Preuß (1991) im Interpretationshandbuch zur KABC: „Die Benennung von Objekten in der Umwelt stellt eine der wichtigsten funktionalen Fertigkeiten bei Kindern dar..." (S. 69).

Seit Templin (1957, zitiert nach Kiese \& Kozielski, 1996, S.7) unterscheidet man zwischen aktivem bzw. expressivem Wortschatz (die Wörter, die ein Kind tatsächlich spricht) und passivem bzw. rezeptivem Wortschatz (die Wörter, die ein Kind versteht). Beide stehen in engem Zusammenhang, auch wenn es wichtige Unterschiede in den Erwerbsprozessen gibt (Benedict, 1979). Der rezeptive Wortschatz entwickelt sich vor dem expressiven und ist Voraussetzung für diesen. Bis zu einem Lebensalter von 24 Monaten ist er wesentlich umfangreicher als der expressive, was in besonderem Maß für die Wortart der Verben gilt (Conti-Ramsden \& Jones, 1997). Diese Diskrepanz verringert sich mit steigendem Wortschatzumfang. Allgemein bestehen große individuelle Unterschiede im Tempo der Entwicklung des Sprachverstehens und der Diskrepanz zwischen Sprachverstehen und Sprachproduktion (Harris, Yeeles, Chasin \& Oakley, 1995).

Der rezeptive Wortschatz verfügt über eine hohe Vorhersagekraft für den expressiven Wortschatz, die spätere allgemeine Sprachentwicklung und die kognitive Entwicklung (vgl. Bornstein \& Haynes, 1998; Grimm, 2000). Andererseits ist bekannt, dass für Eltern der Verstehensbegriff nicht eindeutig ist und diese somit das Sprachverständnis schwer beurteilen können, was die Vorhersagekraft des rezeptiven Wortschatzes einschränkt (Tomasello \& Mervis, 1994). Weiterhin weisen verschiedene Autoren darauf hin, dass der rezeptive Wortschatz ab einem Alter von 1;6 bis 1;8 Jahren mit unterschiedlichen Methoden nicht mehr reliabel erfasst werden kann (Fenson, Dale, Reznick, Thal, Bates, Hartung et al., 1993; Stern 
\& Stern, 1907, 1922). Es ist folglich nicht verwunderlich, dass sich der expressive Wortschatz, und hierbei insbesondere der expressive Wortschatzumfang, als das Maß früher kindlicher Sprachentwicklung im klinischen Alltag und in der entwicklungspsychologischen Forschung durchgesetzt hat.

Der expressive Wortschatz ist konkret und gut zu beobachten (Goorhuis-Brouwer, 1990).

Zumeist wird der expressive Wortschatzumfang als Anzahl aller unterschiedlichen Wörter, die ein Kind spricht (gemessen mit Elternfragebogen [Parent Report]), in der aktuellen Forschung verwendet.

Der expressive Wortschatz gilt derzeit als bester Prädiktor für spätere Sprachentwicklungsstörungen und andere Entwicklungsstörungen (z.B. Fischel, Whitehurst, Caulfield \& DeBaryshe, 1989). Er sagt die spätere grammatische Entwicklung, kognitive Leistungen sowie den Schulerfolg vorher (Fenson, Dale, Reznick, Bates, Thal, \& Pethick, 1994; Thal, Bates, Zappia \& Oroz, 1996; Toppelberg \& Shapiro, 2000). Hierbei verfügt der Wortschatz vor allem zum Zeitpunkt um das zweite Lebensjahr über eine hohe prädiktive Validität (Berglund \& Eriksson, 2000). Kinder, die mit 24 Monaten nicht über 50 Wörter im expressiven Lexikon verfügen und keine Wortkombinationen bilden, werden als „Late Talker" bezeichnet und tragen ein hohes Risiko für die Ausbildung von Sprachentwicklungsund anderen Entwicklungsstörungen. Die aktuelle Forschung befasst sich in besonderem Maße mit der Entwicklung von „Late Talkern“ und prüft die prädiktive Validität dieses Kriteriums. Nur wenige Studien untersuchen die prädiktive Validität des frühen expressiven Wortschatzes für gesunde Stichproben und über eine lange Zeitspanne bezogen auf die normale Sprachentwicklung. Studien für den deutschen Sprachraum sind noch rarer.

Im Rahmen dieser Dissertation soll folgender Frage nachgegangen werden: Sagt der frühe expressive Wortschatz auch für eine Stichprobe deutschsprachiger normal entwickelter Kinder über eine große Zeitspanne von früher Kindheit bis Grundschulalter spätere Sprachleistungen substanziell vorher?

Als international anerkanntes Maß für die frühe grammatische Entwicklung gilt die durchschnittliche Äußerungslänge (Mean Length of Utterance [MLU]). Diese fungiert neben dem expressiven Wortschatzumfang als bewährtes Kriterium für die Diagnose früher Sprachentwicklungsverzögerungen (Late Talker).

Die MLU ist ein globales und sehr nützliches Maß für den grammatischen Fortschritt kleiner Kinder (Szagun, 2001) und gilt als zuverlässiger Indikator verbaler Komplexität und grammatischer Entwicklung in der Kinder- und Erwachsenensprache (Bates \& Carnevale, 
1993). Die MLU im frühen Kindesalter steht in bedeutsamem Zusammenhang zu späterem Sprachoutcome (Walker, Greenwood \& Carta, 1994).

Bisher gibt es keine dokumentierten Studien, die der Forschungsfrage nachgehen, ob die MLU die Vorhersagen späterer Sprachleistungen des Vor- und Grundschulalters aus den frühen Wortschatzdaten verbessern kann. Am vorliegenden Datensatz soll diese Frage überprüft werden.

Als frühes Sprachmaß neben expressivem Wortschatzumfang und MLU wird in vielen Untersuchungen sowie insbesondere im klinischen Setting der „Sprechbeginn“ erhoben, also das Alter, in dem ein Kind erste Worte äußert. Fenson et al. (1994) betonen die große interindividuelle Variabilität im Auftreten des ersten Wortes. In der Literatur lassen sich Zeitpunkte zwischen 8 und 18 Monaten finden (Bloom, Tinker \& Margulis, 1993; Bühler, 1928; McCarthy, 1966). Für die Äußerung erster Wörter sind eine Reihe entscheidender Vorläuferfertigkeiten notwendig wie z.B. Wahrnehmungsleistungen, sprachrelevante soziale Fertigkeiten sowie Gedächtnis- und phonologische Fähigkeiten. Wenig untersucht ist der Zusammenhang des Auftretens erster Worte mit späteren Sprachleistungen bis ins Grundschulalter.

Besteht ein signifikanter Zusammenhang vom Alter, in dem ein Kind erste Worte äußert, mit unterschiedlichen Aspekten von Sprache im frühen Kindesalter bis zum Grundschulalter? Dieses ist eine weitere Forschungsfrage, die im Rahmen dieser Dissertation bearbeitet wird.

Der frühe expressive Wortschatz erfährt in der aktuellen Forschungsliteratur überwiegend eine rein quantitative Betrachtung bezogen auf Vorhersagen von bzw. Zusammenhänge mit späteren Sprachleistungen. Qualitative Analysen des frühen Wortschatzes z.B. hinsichtlich Komposition und Vielfältigkeit zur Vorhersage späterer Sprachleistungen sind rar. Wohl gibt es eine Fülle an (vor allem cross-linguistischen) Untersuchungen zur Zusammensetzung des frühen Wortschatzes insbesondere in Bezug auf die Wortarten und den Ablauf des Erwerbs unterschiedlicher Wortarten (Wortklassenhierarchie; z.B. Maital, Painter, Park, Pascual, Pêcheux, Ruel et al., 2004) für normal entwickelte Kinder, nur selten wird hingegen die prädiktive Validität einzelner Wortarten für die weitere Sprachentwicklung untersucht. In diesem Zusammenhang bildet die Wortart der Verben eine Ausnahme. Verben werden als Schnittstelle zur späteren Grammatikentwicklung betrachtet und als besonders guter Prädiktor für die morphologische Entwicklung bezeichnet (Marchman \& Bates, 1994). Kinder mit „specific language impairment“ (SLI) unterscheiden sich von normal entwickelten Kindern 
insbesondere in Bezug auf die Größe und Diversifizität ihres Verbenlexikons sowie in ihrer Fähigkeit, neue Verben zu erlernen und in ihre Spontansprache zu integrieren (ContiRamsden, 2003).

Die Eignung des Verbenlexikons als Prädiktor für späteres Sprachoutcome wurde selten und nicht über eine große Zeitspanne geprüft. Fraglich ist, ob Verben als Gesamtmenge bzw. bestimmte Arten von Verben zusätzlich zum Gesamtwortschatz weitere substanzielle Varianzaufklärung für spätere Sprachleistungen leisten können und ob für die Teilmenge der Verben ein höherer Zusammenhang zu späterem Sprachoutcome besteht als für den Gesamtwortschatz.

Zudem soll untersucht werden, ob es neben den Verben andere Wortarten (wie z.B. Pronomen und Adjektive) gibt, die als Teilmengen des frühen Gesamtwortschatzes besonders relevante Informationen des frühen Spracherwerbs repräsentieren und somit höhere Zusammenhänge zu späterem Sprachoutcome aufweisen als der gesamte Wortschatzumfang bzw. bedeutsame zusätzliche Varianzaufklärungen für späteres Sprachoutcome erbringen.

Die Komposition des frühen expressiven Wortschatzes lässt sich neben der Zusammensetzung in Bezug auf die Wortarten auch auf die Aspekte Anzahl semantischer Kategorien sowie Inhalts- und Funktionswörter betrachten. Untersuchungen zum Zusammenhang dieser Aspekte mit späterem Sprachoutcome konnten der aktuellen Forschungsliteratur nicht entnommen werden.

Forschungsinteresse dieser Arbeit ist es, zu prüfen, ob die Anzahl semantischer Kategorien sowie die Summe an Inhalts- bzw. Funktionswörtern und deren Verhältnis zueinander in höherem Zusammenhang zu späterem Sprachoutcome stehen als der expressive Wortschatzumfang und ob diese Aspekte zu einer zusätzlichen bedeutsamen Varianzaufklärung späterer Sprachleistungen in Ergänzung zum expressiven Wortschatzumfang führen.

Alle Forschungsfragen vorliegender Arbeit veranschaulicht Tabelle 1. 
Tabelle 1

Forschungsfragen der vorliegenden Arbeit

\begin{tabular}{|c|c|c|}
\hline & Forschungsfrage & Kapitel \\
\hline 1. & $\begin{array}{l}\text { Gibt es bedeutsame Stabilitäten für unterschiedliche Sprachaspekte } \\
\text { von früher Kindheit bis zum Grundschulalter bei normal } \\
\text { entwickelten deutschsprachigen Kindern? }\end{array}$ & 3 \\
\hline 2. & $\begin{array}{l}\text { Sagt der frühe expressive Wortschatz für normal entwickelte } \\
\text { deutschsprachige Kinder spätere Sprachleistungen bis ins } \\
\text { Grundschulalter substanziell vorher? }\end{array}$ & 4 \\
\hline 3. & $\begin{array}{l}\text { Kann die durchschnittliche Äußerungslänge (MLU) in der frühen } \\
\text { Kindheit die Vorhersagen späteren Sprachoutcomes aus den frühen } \\
\text { Wortschatzdaten substanziell verbessern? }\end{array}$ & 5 \\
\hline 4. & $\begin{array}{l}\text { Besteht ein bedeutsamer Zusammenhang von Sprechbeginn und } \\
\text { unterschiedlichen Sprachmaßen für die Altersspanne frühe Kindheit } \\
\text { bis Grundschulalter? }\end{array}$ & 6 \\
\hline 5. & $\begin{array}{l}\text { Können qualitative Analysen des frühen expressiven Wortschatzes } \\
\text { die Vorhersage späterer Sprachmaße aus dem frühen expressiven } \\
\text { Wortschatzumfang (quantitative Betrachtung) bedeutsam } \\
\text { verbessern? }\end{array}$ & 7 \\
\hline 6. & $\begin{array}{l}\text { Stehen die Variablen, die sich aus den qualitativen Analysen des } \\
\text { frühen expressiven Wortschatzes ergeben, in höherem } \\
\text { Zusammenhang zu späterem Sprachoutcome als der expressive } \\
\text { Wortschatzumfang (quantitative Betrachtung)? }\end{array}$ & 7 \\
\hline
\end{tabular}




\section{Methodischer Rahmen der eigenen Untersuchung}

Um die in Kapitel 1 herausgearbeiteten Forschungsfragen auf empirischer Basis beantworten zu können, wurde eine Längsschnittuntersuchung über einen Zeitraum von sechs Jahren realisiert.

\subsection{Zeitlicher Ablauf und Stichprobenbeschreibung}

Ausgangslage für die längsschnittliche Untersuchung von Sprache war die Diplomarbeit der Verfasserin (Bockmann, 2001). Im Rahmen der empirischen Erprobungsphase eines Instruments zur frühen Wortschatzerfassung wurde $2001\left(\mathrm{t}_{0}\right)$ eine Stichprobe von insgesamt 186 Kindern (96 Jungen, 90 Mädchen) im Alter von 16 bis 26 Monaten $(M=20.66$; $S D=$ 3.09) mit den Testbildern zur aktiven Sprache aus der „Münchener Funktionellen Entwicklungsdiagnostik“ (MFED; Hellbrügge, 1994) untersucht sowie deren Eltern die vorläufige Version des Elternfragebogens zur Wortschatzentwicklung (heute: ELAN - Eltern Antworten. Elternfragebogen zur Wortschatzentwicklung im frühen Kindesalter; Bockmann \& Kiese-Himmel, 2006) vorgelegt. Die Kinder kamen aus der ländlichen Umgebung von Kiel, aus Hamburg und Lübeck, aus Hannover und Umgebung sowie aus München. Hierbei lebten $56.1 \%$ der Kinder in der „Stadt“ (definiert mit mehr als 10.000 Einwohnern) und 43.9\% kamen aus ländlichem Umfeld. Sie wurden überwiegend in Kinderarztpraxen (z. T. auch in Krabbelgruppen und Krippen) rekrutiert, wobei darauf geachtet wurde, Praxen und Krippen bzw. Krabbelgruppen auszuwählen, die bzgl. des Bildungsstandes der Eltern gut durchmischt waren. So nahmen z.B. auch Krippen aus sozialen Brennpunktgebieten an der Untersuchung teil. Dennoch ergab sich mit 54.6\% bzw. 57.3\% ein erhöhter Anteil an Müttern und Vätern, die über ein Fachabitur bzw. allgemeine Hochschulreife verfügten.

Für die längsschnittliche Datenerhebung wurde eine Untersuchungsstichprobe von 60 Kindern angestrebt, um eine ausreichend große Stichprobe für die statistischen Datenanalysen zu gewährleisten und gleichzeitig dem Tatbestand Rechnung zu tragen, dass diese Kinder von nur einer Untersucherin in ganz Deutschland verteilt über mehrere Jahre begleitet werden sollten. Ziel war es, jeweils fünf Jungen und fünf Mädchen aus sechs Monatskategorien für die Untersuchung zu rekrutieren. Ausgeschlossen wurden hierbei die Monatskategorien, in denen die Stichprobengröße bzw. die ungleiche Geschlechterverteilung dieses nicht erlaubten sowie Monatskategorien, in denen Stichprobenverzerrungen (sowohl bzgl. des Abschneidens in der MFED als auch im ELAN) nachgewiesen worden waren. Zudem wurde angestrebt, Kinder einer möglichst breiten Altersspanne auszuwählen, damit sowohl Kinder, die vor bzw. am Anfang des Wortschatzspurtes (von 18 bis 21 Monaten) standen, als auch solche, die ihn 
schon deutlich abgeschlossen hatten, in die Untersuchung einbezogen werden konnten. Auf diese Weise sollte eine große Variabilität in den frühen Wortschatzdaten bezogen auf quantitative und qualitative Aspekte als Ausgangslage für alle weiteren Berechnungen garantiert werden. Die Kinder wurden zufällig ausgewählt. Ihre Eltern wurden telephonisch für das Forschungsvorhaben geworben und in einem ausführlichen Elternbrief über die inhaltlichen und zeitlichen Aspekte des Projektes informiert. Nicht erreicht wurden sechs Kinder (verteilt auf drei Monatskategorien), da diese aus einer Münchener Kinderarztpraxis stammten, deren komplettes Datensystem aufgrund eines technischen Defektes für Wochen zusammengebrochen war. Eine Mutter entschied sich gegen die Teilnahme am Längsschnittprojekt ohne Nennung eines Grundes. Hier konnte jedoch ein Kind gleichen Alters und Geschlechtes nachrekrutiert werden. Drei Kinder wurden auf die Nachfrage ihrer interessierten Eltern zusätzlich aufgenommen. So ergab sich als Untersuchungsstichprobe für den ersten Messzeitpunkt ( $\left.\mathrm{t}_{1}\right)$ des Promotionsprojektes (2003) eine Stichprobe von 57 Kindern (28 Jungen, 29 Mädchen) im Alter von 41 bis 53 Monaten, die ein mittleres Alter von 47.79 Monaten $(S D=2.82)$ aufwiesen. Die Altersverteilung dieser 57 Kinder $\mathrm{zu} \mathrm{t}_{1}$ ist Tabelle $2 \mathrm{zu}$ entnehmen.

Tabelle 2

Altersverteilung der Untersuchungsstichprobe zum Zeitpunkt $t_{1}$

\begin{tabular}{|ccc|}
\hline Monate & Häufigkeit & Prozent \\
\hline 41 & 1 & 1,8 \\
42 & 2 & 3,5 \\
43 & 2 & 3,5 \\
44 & 1 & 1,8 \\
45 & 6 & 10,5 \\
46 & 6 & 10,5 \\
47 & 7 & 12,3 \\
48 & 8 & 14,0 \\
49 & 7 & 12,3 \\
50 & 7 & 12,3 \\
51 & 4 & 7,0 \\
52 & 5 & 8,8 \\
53 & 1 & 1,8 \\
\hline Gesamt & 57 & 100,0 \\
\hline
\end{tabular}






Tabelle 3

Altersverteilung der Untersuchungsstichprobe zum Zeitpunkt $t_{0}$

\begin{tabular}{|ccc|}
\hline Monate & Häufigkeit & Prozent \\
\hline 16 & 1 & 1,8 \\
17 & 1 & 1,8 \\
18 & 8 & 14,0 \\
19 & 8 & 14,0 \\
21 & 8 & 14,0 \\
22 & 10 & 17,5 \\
24 & 11 & 19,3 \\
25 & 10 & 17,5 \\
\hline Gesamt & 57 & 100,0 \\
\hline
\end{tabular}

Betrachtet man den Bildungsabschluss der Mütter und Väter in dieser Stichprobe, ergibt sich folgendes Bild: 50.8\% der Mütter und 54.6\% der Väter besitzen einen Hauptschul- bzw. Realschulabschluss und 49.2\% der Mütter sowie 45.4\% der Väter gaben als Schulabschluss Fachabitur bzw. allgemeine Hochschulreife an. Aus städtischem Wohngebiet (mehr als 10.000 Einwohner) kamen 30.4\% der Kinder und 69.6\% der Kinder kamen vom Land. Trotz der zum Teil geringen Stichprobengröße wurden konfirmatorisch für alle Sprachdaten zu $\mathrm{t}_{1}$ und zum frühen Wortschatz $\mathrm{zu} \mathrm{t}_{0}$ (Prozentrang im ELAN) t-Tests gerechnet, die in keinem Fall einen signifikanten Einfluss des Wohnortes Stadt vs. Land (in der hier verwendeten Definition) auf die gezeigten Sprachleistungen ergaben.

Der mittlere Zeitabstand zwischen $\mathrm{t}_{0}$ und $\mathrm{t}_{1}$ betrug 26.19 Monate $(S D=1.63$, Min $=21$, Max =29). Für alle teilnehmenden Kinder gilt zu allen Messzeitpunkten, dass Berichte mit den Untersuchungsergebnissen an die Eltern und auf Wunsch an den behandelnden Kinderarzt bzw. Therapeuten oder die Erzieherin geschickt und die entsprechenden therapeutischen und beratenden Maßnahmen schriftlich und ggf. zusätzlich im Gespräch empfohlen wurden. Diese Informationen wurden dokumentiert und im weiteren Verlauf der Längsschnittuntersuchung kontinuierlich erfragt und aktualisiert. So erfuhren alle Kinder die gleiche Behandlung und diese insbesondere ethisch befriedigende Vorgehensweise gekoppelt mit der beruflichen Doppelqualifikation der Untersucherin (Logopädin und Diplom- 
Psychologin) mögen zu der hohen Mitarbeitsbereitschaft und geringen Abbruchquote von vier Kindern über 6 Jahre innerhalb der Stichprobe geführt haben. Bei nur einem dieser vier Kinder hatten die Eltern die Mitarbeit an der Studie wegen der Geburt des Geschwisterkindes und Umzuges direkt abgesagt. Die anderen drei Kinder schieden aufgrund von langfristiger Erkrankung, Umzug und ausgeprägter Verhaltensauffälligkeit (Verdacht auf elektiven Mutismus) aus der Stichprobe aus.

Der zweite Messzeitpunkt erfolgte im Jahr 2003. Die Stichprobe umfasste 54 Kinder (26 Jungen, 28 Mädchen). Die Kinder waren im Durchschnitt 60.07 Monate alt ( $S D=2,81$, Min = 53, Max $=65)$. Der mittlere Zeitabstand zwischen $\mathrm{t}_{1}$ und $\mathrm{t}_{2}$ betrug 12.26 Monate $(S D=0.48$, Min = 11, Max = 13). Die genaue Altersverteilung der Stichprobe $\mathrm{zu} \mathrm{t}_{2}$ ist in Tabelle 4 dargestellt.

Tabelle 4

Altersverteilung der Untersuchungsstichprobe zum Zeitpunkt $t_{2}$

\begin{tabular}{|ccc|}
\hline Monate & Häufigkeit & Prozent \\
\hline 53 & 1 & 1,9 \\
54 & 1 & 1,9 \\
55 & 3 & 5,6 \\
56 & 1 & 1,9 \\
57 & 3 & 5,6 \\
58 & 7 & 13,0 \\
59 & 4 & 7,4 \\
60 & 5 & 9,3 \\
61 & 13 & 24,1 \\
62 & 5 & 9,3 \\
63 & 5 & 9,3 \\
64 & 5 & 9,3 \\
65 & 1 & 1,9 \\
\hline Gesamt & 54 & 100,0 \\
\hline
\end{tabular}

Der dritte Messzeitpunkt fand im Jahr 2006 statt. Im Unterschied zu den vorherigen Untersuchungen waren die Versuchsleiterinnen zwei Mitarbeiterinnen der Abteilung Pädagogische Psychologie und Entwicklungspsychologie des psychologischen Instituts der Georg-August-Universität Göttingen. Die Verfasserin übernahm die telephonische Rekrutierung aller teilnehmenden Kinder und unterstütze die Datenerhebung mittels 
Durchführung einer Untersuchung in München, Anwesenheit bei einer weiteren Untersuchung bei einem besonders schüchternen Kind sowie telephonischer Beratung bzgl. der Rückmeldung der Ergebnisse bei ausgewählten Kindern. Ein Kind konnte wegen Umzugs nicht erreicht werden, so dass sich die Stichprobengröße auf 53 Kinder (25 Jungen und 28 Mädchen) verringerte. Die Kinder hatten $\mathrm{zu} \mathrm{t}_{3}$ ein mittleres Alter von 93.6 Monaten $(S D=$ 3.44, Min = 85, Max =99). Der mittlere Zeitabstand zwischen $t_{2}$ und $t_{3}$ betrug 33.47 Monate $(S D=2.37, \operatorname{Min}=27, \operatorname{Max}=36)$. Die genaue Altersverteilung zu t $\mathrm{t}_{3}$ zeigt Tabelle 5.

Tabelle 5

Altersverteilung der Untersuchungsstichprobe zum Zeitpunkt $t_{3}$

\begin{tabular}{|ccc|}
\hline Monate & Häufigkeit & Prozent \\
\hline 85 & 2 & 3,8 \\
86 & 1 & 1,9 \\
87 & 1 & 1,9 \\
88 & 1 & 1,9 \\
89 & 2 & 3,8 \\
90 & 3 & 5,7 \\
91 & 4 & 7,5 \\
92 & 2 & 3,8 \\
93 & 3 & 5,7 \\
94 & 8 & 15,1 \\
95 & 7 & 13,2 \\
96 & 11 & 20,8 \\
97 & 4 & 7,5 \\
98 & 2 & 3,8 \\
99 & 2 & 3,8 \\
\hline Gesamt & 53 & 100,0 \\
\hline
\end{tabular}

Tabelle 6 stellt noch einmal zusammenfassend den Untersuchungsplan im zeitlichen Ablauf über alle vier Messzeitpunkte $\left(\mathrm{t}_{0}\right.$ bis $\left._{3}\right)$ dar. 
Tabelle 6

Beschreibung des Untersuchungsplans

\begin{tabular}{lllll} 
& $\mathbf{t}_{\mathbf{0}}$ & $\mathbf{t}_{\mathbf{1}}$ & $\mathbf{t}_{\mathbf{2}}$ & $\mathbf{t}_{\mathbf{3}}$ \\
\hline $\mathbf{J a h r}$ & 2001 & 2003 & 2004 & 2006 \\
$\boldsymbol{N}$ & 57 & 57 & 54 & 53 \\
Alter & $21.6(2.6)$ & $47.8(2.8)$ & $60.1(2.8)$ & $93.6(3.4)$ \\
Geschlecht & $28 / 29$ & $28 / 29$ & $26 / 28$ & $25 / 28$
\end{tabular}

Anmerkungen $. \mathrm{t}_{0}-\mathrm{t}_{3}=$ Erhebungszeitpunkte, $N=$ Stichprobengröße, Alter (Mittelwert in Monaten, in Klammern Standardabweichungen), Geschlecht (Jungen / Mädchen)

\subsection{Untersuchungsverfahren}

Zur Untersuchung der expressiven Wortschatzleistungen, des Satzgedächtnisses sowie der expressiven und rezeptiven grammatischen Fähigkeiten kam über die vier Erhebungszeitpunkte eine Reihe von Untersuchungsverfahren zum Einsatz, welche Tabelle 7 veranschaulicht. 
Tabelle 7

Überblick über die verwendeten Untersuchungsverfahren von $t_{0}$ bis $t_{3}$

$\begin{array}{llll}\mathbf{t}_{\mathbf{0}} \mathbf{( 2 0 0 1 )} & \mathbf{t}_{\mathbf{1}} \mathbf{( 2 0 0 3 )} & \mathbf{t}_{\mathbf{2}} \mathbf{( 2 0 0 4 )} & \mathbf{t}_{\mathbf{3}}(\mathbf{2 0 0 6}) \\ N=57 & N=57 & N=54 & N=53\end{array}$

Alter:

21.6 Monate $\quad$ 47.8 Monate $\quad$ 60.1 Jahre Monate

Wortschatz:

ELAN AWST 3-6

AWST 3-6

HAWIK III (WS)

$(N=38) \quad(N=54)$

Grammatik (expressiv):

MLU

SETK 3-5 (MR)

SETK 3-5 (MR)

HSET (PS)

Grammatik (rezeptiv):

SETK 3-5 (VS) SETK 3-5 (VS) HSET (VS)

Satzgedächtnis:

SETK 3-5 (SG) SETK 3-5 (SG) HSET (SG)

Anmerkungen. $\mathrm{t}_{0}-\mathrm{t}_{3}=$ Erhebungszeitpunkte, $N$ und $n=$ Stichprobengröße, Alter: Mittelwert in Monaten, ELAN = Elternfragebogen zur Wortschatzentwicklung im frühen Kindesalter, AWST 3-6 = Aktiver Wortschatztest für 36-jährige Kinder, HAWIK III (WS) = Untertest „Wortschatz“ aus dem Hamburg-Wechsler-Intelligenztest für Kinder III, MLU = Mean Length of Utterances, SETK 3-5 = Sprachentwicklungstest für drei- bis fünfjährige Kinder, SETK 3-5 (MR) = Untertest „Morphologische Regelbildung“ aus dem SETK 3-5, SETK 3-5 (SG) = Untertest „Satzgedächtnis“ aus dem SETK 3-5, SETK 3-5 (VS) = Untertest „Verstehen von Sätzen“ aus dem SETK 3-5, HSET = Heidelberger Sprachentwicklungstest, HSET (VS) = Untertest „Verstehen grammatischer Strukturformen“ aus dem HSET, HEST (PS) = Untertest „Plural-Singular-Bildung“ ”aus dem HSET, HSET (IS) = Untertest „Imitation grammatischer Strukturformen“ aus dem HSET

\section{Wortschatz}

Die Erfassung des Wortschatzes ist eine methodisch nicht einfach zu bewältigende Aufgabe. Jede Operationalisierung ermöglicht lediglich die Betrachtung von Teilaspekten des Wortschatzes. So werden bei der Operationalisierung „Bildbenennung“ zur Erfassung des expressiven Wortschatzes nur Ausschnitte des Wortschatzes (über die Auswahl des dargebotenen Bildmaterials) untersucht. Wählt man die Zuordnung von semantisch ähnlichen Wörtern bzw. Gegensätzen oder Oberbegriffen zu vorgegebenen Wörtern und erfasst somit eher den passiven Wortschatz, ist auch hier nur ein Ausschnitt des Gesamtwortschatzes darstellbar. Der Wortschatzumfang lässt sich immer nur hochrechnen auf der Grundlage einer 
ausgewählten Anzahl an Bildern bzw. Wörtern. Entscheidend für die Güte der Messung ist die Repräsentativität des ausgewählten Bild- und Wortmaterials.

$\mathrm{Zu} \mathrm{t}_{0}$ wurde der Elternfragebogen zur Wortschatzentwicklung im frühen Kindesalter (heute: ELAN - Eltern Antworten; Bockmann \& Kiese-Himmel, 2006) als anerkanntes Parent Report Verfahren zur Erfassung des expressiven Wortschatzes in der frühen Kindheit (für den deutschen Sprachraum) eingesetzt. Der Vorteil dieses Verfahrens liegt in der Unabhängigkeit der Ergebnisse von der direkten Untersuchung des Kindes, welche in diesem frühen Alter je nach Verfassung des Kindes und Kontakt zum fremden Versuchsleiter unterschiedlich reliable und objektive Aussagen bzgl. der frühen Sprachleistungen zulassen würde. Es liegen Normen (Prozentränge und T-Werte) von 270 normal entwickelten Kindern aus 14 Bundesländern getrennt nach zwei Altergruppen vor (16 bis 20 Monate für Jungen und Mädchen sowie 21 bis 26 Monate getrennt nach Jungen und Mädchen). Der Fragebogen erfasst zum einen soziodemographische und sozialanamnestische Daten sowie Angaben zur Gesundheitsentwicklung des Kindes. Zum anderen erhebt er mit einer Vokabelcheckliste zum Ankreuzen der aktiv vom Kind gesprochenen Wörter strukturiert in 17 semantischen Kategorien den Umfang des expressiven Wortschatzes (Min = 0, Max =250). Darüber hinaus besteht auf der letzten Seite die Möglichkeit zur freien Angabe von weiteren Wörtern, welche das Kind spricht und es werden Angaben zur syntaktischen Entwicklung erfragt. Der ELAN gilt als objektives, reliables und valides Verfahren zur Erfassung des frühen Wortschatzes. So beliefen sich die Werte für die Retest-Reliabilität (Zeitspanne 10-14 Tage) auf .98 und für die innere Konsistenz konnte ein Cronbachs Alpha von .99 ermittelt werden. Die Validität des ELANs konnte u. a. über die Korrelation der Ergebnisse mit dem Abschneiden in der Münchener Funktionellen Entwicklungsdiagnostik (Hellbrügge, 1994) als bewährtem Verfahren abgesichert werden. Hier belief sich die Korrelation auf .89.

Als standardisiertes Verfahren zur Erfassung des expressiven kindlichen Wortschatzes zu $\mathrm{t}_{1}$ und $\mathrm{t}_{2}$ wurde der „Aktive Wortschatztest für 3-6-jährige Kinder“ (AWST 3-6; Kiese \& Kozielski, 1996) eingesetzt. Dieser erhebt den expressiven Wortschatz der Kinder über die Bildbenennung von 82 in Schwarz-Weiß-Zeichnungen dargestellten Wörtern (64 Substantive, 17 Verben und 1 Adjektiv), die in steigender Schwierigkeit hoch vertraute bis wenig vertraute Gegenstände aus dem Umfeld der Kinder darstellen (z.B. Kopf, Arm und Mikrophon). Für den Summenwert aller korrekt benannten Bilder $($ Max $=82)$ lassen sich getrennt nach Jungen und Mädchen sowie Altersgruppen Prozentränge ablesen, wobei ergänzt werden soll, dass diese Normen aus heutiger Sicht als veraltet zu bezeichnen sind. Der AWST 3-6 gilt als 
objektiv, zuverlässig und valide. So wird von einer Split-half-Reliabilität von .94 berichtet und bezüglich der Validität konnte das Vorliegen von diskriminanter als auch konvergenter Validität nachgewiesen werden.

Wichtig anzumerken ist, dass gerade für sehr junge Kinder das Bildmaterial des AWST 36 wenig ansprechend und z. T. nur schwer zu erkennen ist. Auch waren einige Begriffe bzw. Darstellungen nicht mehr zeitgemäß (z.B. „Telegrafenmast“ als Begriff oder „Mikrophon“ mit Schnur in der bildlichen Darstellung). Dieses hat aufgrund des Abbruchkriteriums zur Folge, dass insbesondere junge Kinder in der Bearbeitung des AWST 3-6 nicht weit kommen, also nicht entsprechend ihrer eigentlichen Leistungen bewertet werden. Leider stand auch zu $\mathrm{t}_{2}$ der überarbeitete „Aktiver Wortschatztest für 3- bis 5-jährige Kinder“ (AWST-R; KieseHimmel, 2005), in dem die farbigen Photos wesentlich ansprechender und die Begriffsauswahl dem Zeitgeist angemessener ist, noch nicht zur Verfügung.

Für die Erfassung des expressiven Wortschatzes zu t 3 wurde der Untertest „Wortschatz" aus dem „Hamburg-Wechsler-Intelligenztest für Kinder III“ (HAWIK III; Tewes, Rossmann \& Schallberger, 1999) ausgewählt (im Folgenden abgekürzt mit HAWIK WS). Das Konstrukt „Wortschatz“ wird über die mündliche Definition von vorgegebenen Begriffen operationalisiert. Im HAWIK WS müssen die Kinder Fragen beantworten wie z.B. „Was ist eine Insel?“ oder „Was bedeutet abstrakt?“. Die Antwort wird je nach Definitionsgenauigkeit mit 0 bis 2 Punkten bewertet, so dass ein Kind maximal eine Punktsumme von 60 erreichen kann. Der HAWIK WS erfasst den Umfang des Wortschatzes und gibt Auskunft über die allgemeine sprachliche Entwicklung, insbesondere im Hinblick auf den Bestand an sprachlichen Kenntnissen. Die Leistungen sind auch abhängig vom Lern- und Erinnerungsvermögen des Kindes sowie von der Entwicklung der Begriffsbildung. Kulturelle Einflüsse, Interessen, schulische Lernvorgänge sowie Lese- und Sprachgewohnheiten innerhalb der Familie beeinflussen zudem den Bestand an Wortkenntnissen. Die Objektivität des Verfahrens wird durch präzise formulierte Instruktionen, Auswertungskriterien und das Vorliegen von Normen gesichert. Für den Untertest „Wortschatz“ stehen ausführliche Auswertungskriterien und Beispiele zur Verfügung, um die 0- bis 2-Punkte-Bewertung objektiv vornehmen zu können. Die Reliabilität des Untertests „Wortschatz“ wird über die Split Half Reliabilität mit .77 bis .81 für die sechs- bis achtjährigen Kinder geschätzt. Den Angaben zur faktoriellen Validität ist zu entnehmen, dass der Untertest „Wortschatz“ über alle Altersgruppen am höchsten auf dem Verbalfaktor lädt. Die Ladungen auf dem Verbalfaktor erreichen für 6- bis 10-jährige Kinder Werte von .71 bis .79. Im Gegensatz 
hierzu errechnen sich für die Ladungen auf dem Handlungsfaktor Werte von .20 bis .26. Auf den Faktor „sprachliches Verständnis“ z.B. lädt der Untertest „Wortschatz“ für 6- bis 10jährige Kinder mit .72 bis .86. Ein altersgerechtes Verfahren, welches den Wortschatz über Bildbenennung (wie zu $\mathrm{t}_{1}$ und $\mathrm{t}_{2}$ ) erhebt, existiert für den deutschen Sprachraum nicht.

\section{Grammatik (expressiv)}

$\mathrm{Zu} \mathrm{t}_{0}$ wurde mittels Elternangaben im ELAN die durchschnittliche Äußerungslänge als anerkanntes Maß für die frühe grammatische Entwicklung bestimmt. Auf der letzten Seite des Fragebogens wurden die Eltern gebeten, Angaben zur syntaktischen Entwicklung ihres Kindes zu machen (z.B. „Bitte schreiben Sie abschließend drei typische Beispiele für Äußerungen Ihres Kindes auf"). Aus den typischen Satzbeispielen wurde die durchschnittliche Äußerungslänge in Worten errechnet. Angaben zur Reliabilität und prädiktiven Validität der MLU sind Kapitel 5.1 zu entnehmen.

Als standardisierter Test zur Erfassung der expressiven grammatischen Fähigkeiten wurde der „Sprachentwicklungstest für drei- bis fünfjährige Kinder“ (SETK 3-5; Grimm, 2001) zu t und $t_{2}$ verwendet. Für den SETK 3-5 sind im Manual Cronbachs-Alpha-Werte von .62 bis .89 für die interne Konsistenz angegeben. Das Vorliegen einer hohen Objektivität wurde unter anderem über die Interrater-Übereinstimmung von z.B. $90.1 \%$ für den besonders anspruchsvoll auszuwertenden Untertest „Enkodieren Semantischer Relationen“ nachgewiesen. Das Bestehen von Validität wurde über die hohen Interkorrelationen der Untertests, die Altersabhängigkeit derselbigen und die geringe Korrelation mit konstruktfernen Variablen abgesichert. Der Untertest „Morphologische Regelbildung“ (MR) prüft bei allen Kindern die Fähigkeit Pluralformen morphologisch zu markieren, was als stellvertretende Fähigkeit für die expressiven grammatischen Leistungen gilt. Im Unterschied zu den dreijährigen Kindern müssen die älteren Kinder zusätzlich die Pluralmarkierung von acht Kunstwörtern leisten. Bei ihnen wird also auch die Fähigkeit geprüft, eine Pluralregel korrekt anzuwenden. Dreijährige Kinder können maximal 20 Punkte, Vierjährige und ältere Kinder maximal 36 Punkte erreichen.

Als standardisiertes Verfahren zur Erfassung der expressiven grammatischen Leistungen zu t $t_{3}$ wurde der „Heidelberger Sprachentwicklungstest“ (HSET; Grimm \& Schöler, 1991) eingesetzt, der für das Alter von drei bis neun Jahren normiert ist. Der Untertest „PluralSingular-Bildung“ (PS) untersucht, ob die semantische Unterscheidung in Einzahl vs. 
Mehrzahl regelhaft morphologisch gekennzeichnet werden kann. Es werden wie beim Untertest MR aus dem SETK 3-5 hauptsächlich Kunstwörter vorgegeben. Somit wird in der längsschnittlichen Datenbetrachtung der Untertest PS ( $\left.\mathrm{zu} \mathrm{t}_{3}\right)$ als Fortführung der Messung des Konstrukts „expressive grammatische Fähigkeiten“ gewertet. Erzielt werden können maximal 36 Rohwertpunkte.

Der HSET gilt als objektives, reliables und valides Verfahren zur Erfassung sprachlicher Leistungen im Kindesalter. So konnten z.B. bezogen auf die Objektivität Interraterkorrelationen von .99 und .98 für die Untertests PS und IS ermittelt werden. Die Split Half Reliabilität der hier verwendeten Untertests VS, IS und PS beläuft sich auf Werte von .90 - .92. Zur Beschreibung der internen Validität wurden Interkorrelationen der Untertests VS, IS und PS von .51 - .68 dokumentiert und bezüglich der externen Validität konnten erwartungskonform Altersabhängigkeit für alle Untertests und Milieuabhängigkeit nachgewiesen werden. Die diskriminante Validität wurde nur ansatzweise untersucht. Auch die Normen des HSET müssen aus heutiger Sicht als veraltet bezeichnet werden. Aktuelle Untersuchungen von Rohwerteverteilungen des HSET ergaben jedoch annähernde Entsprechungen mit den Rohwerteverteilungen des HSET von 1975 (H. Schöler, persönliche Mitteilung 4.3.2007).

\section{Grammatik (rezeptiv)}

Für den Erhebungszeitpunkt $\mathrm{t}_{0}$ wurden keine rezeptiven grammatischen Fähigkeiten erhoben, weil ein entsprechendes standardisiertes Messinstrument für das junge Alter von 16 bis 26 Monaten nicht zur Verfügung stand.

$\mathrm{Zu} \mathrm{t}_{1}$ und $\mathrm{t}_{2}$ wurde der Untertest „Verstehen von Sätzen“ (VS) aus dem SETK 3-5 verwendet, um rezeptive grammatische Fähigkeiten zu erheben. Dieser prüft mittels der Methode Bildauswahl und mittels Manipulationsaufgaben die Fähigkeit, Sätze unterschiedlicher Komplexität zu verstehen. Dreijährigen Kindern werden neun Bildkarten präsentiert, die in unterschiedlicher Schwierigkeit die Identifikation von Negation, Präposition, Prädikat und Subjekt verlangen (z.B. „Die Kinder sitzen unter dem Tisch.“). Das Kind muss auf einer Bildkarte die korrekte bildliche Darstellung des vorgesprochenen Satzes aus vier Darstellungen (drei Ablenker) auswählen. Dann werden zehn Manipulationsaufgaben vorgegeben, bei denen das Kind grammatisch einfache bis komplexe Satzkonstruktionen nachspielen muss (z.B. „Bevor du mir den Sack gibst, hol alle Stifte raus.“). So können dreijährige Kinder einen Maximalwert von 19 Punkten erzielen. Vier- bis fünfjährigen 
Kindern werden ausschließlich 15 Manipulationsaufgaben (Maximalwert $=15$ ) präsentiert, die eine noch höhere Satzkomplexität aufweisen (z.B. „Zeig mir: Der gelbe Ball, den der weiße Ball anstößt, fällt vom Tisch.“)

$\mathrm{Zu} \mathrm{t}_{3}$ wurde der Untertest „Verstehen grammatischer Strukturformen“ (VS) aus dem HSET verwendet. Dieser wird, wie der Untertest „Verstehen von Sätzen“ aus dem SETK 3-5, ebenfalls mit VS abgekürzt. Deshalb wird im Folgenden HSET VS als Abkürzung für den Untertest „Verstehen grammatischer Strukturformen“ aus dem HSET verwendet. Der Untertest HSET VS prüft die Fähigkeit, Sätze unterschiedlicher Komplexität zu verstehen und gibt Auskunft über das erworbene linguistische Regelwissen. Die Ergebnisse im HSET VS werden mit den vorherigen Ergebnissen im Untertest „Verstehen von Sätzen“ aus dem SETK 3-5 in Beziehung gesetzt. Die maximale Punktsumme beträgt 17 Rohpunktwerte.

\section{Satzgedächtnis}

Im Rahmen dieser Arbeit soll Satzgedächtnis nicht dem Konstrukt „expressive grammatische Fähigkeiten“ zugeordnet werden. Vielmehr wird Satzgedächtnis als ein Maß der Sprachentwicklung „,im weiteren Sinne“ verstanden, was kein eigenständiges theoretisches Konstrukt meint, sondern ein metrisches Maß, welches besonders von kognitiven Faktoren abhängig ist. Aus diesem Grund wird Satzgedächtnis in Abgrenzung von den drei Maßen der Sprachentwicklung „im engeren Sinne“ (Wortschatz und rezeptive sowie expressive grammatische Fähigkeiten) als eigenständiger sprachlicher Aspekt betrachtet, der sowohl expressive grammatische Fähigkeiten als auch Arbeitsgedächtnisleistungen repräsentiert. Die Betrachtung von Satzgedächtnis als überwiegend sprachlichem Faktor wird fundiert durch Ergebnisse aus der aktuellen Forschungsliteratur, in der mehrfach dokumentiert ist, dass Satzgedächtnisergebnisse in Faktorenanalysen höher auf den Faktor Sprache als auf den Faktor Arbeitsgedächtnis laden (z.B. Marx, 2006). Dass sich das Nachsprechen von Sätzen als geeignet erwiesen hat, Sprach- und Schriftspracherwerbsprobleme von unauffälligen Sprachentwicklungsbefunden abzugrenzen (Knapp, Antas, Becker-Mrotzek, Deppermann,

Göpferich, Grabowski et al., 2004), betont die Relevanz dieses Faktors als Untersuchungsgegenstand für Studien zum Spracherwerb.

Das Satzgedächtnis wurde $\mathrm{zu} \mathrm{t}_{0}$ nicht erhoben, da kein geeignetes Verfahren für den Altersbereich der Untersuchungsstichprobe zu diesem Messzeitpunkt zur Verfügung stand. 
$\mathrm{Zu} \mathrm{t}_{1}$ und $\mathrm{t}_{2}$ wurde das Satzgedächtnis über den Untertest „Satzgedächtnis“ (SG) aus dem SETK 3-5 als ein Gedächtnismaß für die Nutzungsfähigkeit von grammatischen Kenntnissen zur Reproduktion von 15 Sätzen erhoben. Bei korrekter Reproduktion aller Sätze konnte ein Maximalwert von 119 erzielt werden. Die Sätze unterschieden sich bzgl. ihrer Länge (6 bis 10 Wörter) und ihrer inhaltlichen Sinnhaftigkeit (z.B. „Die Ente sitzt neben dem Auto“ vs. „Anna bellt, nachdem sie getrunken wurde.“). Es wird davon ausgegangen, dass höhere linguistische Kenntnisse auch zu besseren Reproduktionsleistungen führen. Hierbei soll die Ausschaltung des allgemeinen Weltwissens (inhaltlich sinnlose Sätze) Aussagen über die Beherrschung der formal linguistischen Regeln ermöglichen.

Der Untertest „Imitation grammatischer Strukturformen“ (IS) erfasst über das Nachsprechen von unterschiedlich komplexen Satzstrukturen das Satzgedächtnis $\mathrm{zu}_{3}$ und wird als Entsprechung des Untertests SG aus dem SETK 3-5 in der längsschnittlichen Datenbetrachtung betrachtet. In Ergänzung zum Untertest „Verstehen von Sätzen“ aus dem HSET gibt der Untertest IS Aufschluss über die Anwendung von entwicklungsspezifischen Regeln. Die zu erreichende Höchstpunktzahl im Untertest IS beträgt 24 Rohpunktwerte.

\subsection{Durchführung}

$\mathrm{Zu} \mathrm{t}_{0}$ füllten alle Eltern den „Elternfragebogen zur Wortschatzentwicklung des Kindes“ (Bockmann, 2001) aus, der seit Oktober 2006 unter dem Namen ELAN veröffentlicht ist. Die Testbilder zur aktiven Sprache aus der MFED wurden allen Kindern zur Benennung vorgelegt. Die Untersuchungen der Kinder mit der MFED sowie das Ausfüllen der Fragebögen durch die Eltern fanden in unterschiedlichen Settings (z.B. Kinderarztpraxis, zu Hause oder Krabbelgruppe) statt. $\mathrm{Zu}$ den weiteren Messzeitpunkten wurde angestrebt, die gleichen Untersuchungssettings wie $\mathrm{zu} \mathrm{t}_{0}$ beizubehalten. Die meisten Untersuchungen fanden im häuslichen Umfeld des Kindes statt; hier zeigten sich Eltern und Kinder besonders vertraut, belastbar und mitarbeitsbereit.

Zum Untersuchungszeitpunkt $t_{1}$ füllten die Eltern der 57 Kinder einen Anamnesefragebogen aus. Die Kinder wurden mit dem AWST 3-6 untersucht. Für diesen wurde nach den ersten drei Untersuchungen entschieden, ihn nur bei besonders kooperativen und aufmerksamen Kindern durchzuführen, da er in hohem Maße anspruchsvoll für junge Kinder und nur wenig motivierend war. Bei 38 Kindern wurde der AWST 3-6 zu t durchgeführt. 
Als weiterer standardisierter Test zur Erfassung der Sprachentwicklung wurde der SETK 3-5 durchgeführt. Dieser sieht unterschiedliche Untertests für die Altersstufen der noch dreijährigen und schon vierjährigen und fünfjährigen Kinder vor. Hieraus ergab sich zu $t_{1}$ eine Teilung der Stichprobe. Den 26 dreijährigen Kindern wurden die Untertests „Verstehen von Sätzen“ (VS) und „Morphologische Regelbildung“ (MR) nicht aber der Untertest „Satzgedächtnis“ (SG) vorgelegt. Im Gegensatz hierzu wurden mit den 31 vierjährigen Kindern die Untertests VS, MR und SG durchgeführt. Zudem bestehen die Untertests MR und VS für dreijährige Kinder aus anderen Items als die für vierjährige Kinder und sind somit bezogen auf ihre Rohsummen nicht vergleichbar.

$\mathrm{Zu} \mathrm{t}_{2}$ füllten alle Eltern erneut den Anamnesebogen (wie $\mathrm{t}_{1}$ ) aus, der durch 16 Fragen zum kindlichen Verhalten und acht Fragen zum Familienalltag erweitert worden war. Als standardisierte Verfahren zur Erfassung der Sprachentwicklung wurden erneut der AWST 3-6 und der SETK 3-5 durchgeführt. Zu diesem Messzeitpunkt waren alle Kinder mindestens vier Jahre alt, so dass allen 54 Kindern die gleichen Untertests in gleicher Weise präsentiert werden konnten.

$\mathrm{Zu} \mathrm{t}_{3}$ füllten die Eltern einen Anamnesebogen aus und den Kindern wurden Fragen zu ihrem unmittelbaren Alltag gestellt (z.B. zu ihrem Lieblingsfach in der Schule bzw. Lieblingsfernsehsendung). Optional konnten die Kinder eine Zeichnung von sich anfertigen und drei Wünsche nennen, die sie (wenn eine Fee sie danach fragen würde) am liebsten erfüllt bekommen hätten. Die Antworten wurden in einem „Kinderfragebogen“ dokumentiert. Da die Kinder mit einem nun durchschnittlichen Alter von 7;10 Jahren durchweg zu alt für den SETK 3-5 und den AWST 3-6 waren, wurde zur Erfassung des expressiven Wortschatzes der Untertest „Wortschatz“ aus dem HAWIK III (HAWIK WS) durchgeführt. Darüber hinaus wurden den Kindern die Untertests HSET VS, PS und IS vorgelegt.

Da zu den Erhebungszeitpunkten $t_{1}, t_{2}$ und $t_{3}$ neben den Sprachmaßen weitere Maße der kindlichen Entwicklung (z.B. Arbeitsgedächtnismaße, Intelligenzleistungen sowie Lese- und Rechtschreibfähhigkeiten) erfasst wurden, die in den hier vorgelegten Analysen nicht berücksichtigt werden, entstand für die Stichprobe der untersuchten Kinder ein großer Umfang an Belastungen in der Untersuchungssituation. Die Untersuchungen $\mathrm{zu} \mathrm{t}_{3}$ dauerten z.B. durchschnittlich 90 bis 120 Minuten. Um diesem Tatbestand Rechnung zu tragen, wurde im Vorfeld eine Reihenfolge für den Ablauf der Untersuchung festgelegt, welche das Maß der 
Belastung durch Abwechslung und Belohnungszeiten möglichst gering halten und gleichzeitig den Grad an Motivation und Kooperationsbereitschaft erhöhen bzw. stabil halten sollte. Die vorab festgelegte Reihenfolge wurde für alle Kinder über alle Messzeitpunkte beibehalten. Dennoch gelang es nicht allen Kindern alle Untersuchungsteile komplett mitzumachen. Mehrere Kinder verweigerten z.B. $\mathrm{zu} \mathrm{t}_{2}$ die komplette Durchführung des als sehr belastend empfundenen Untertests „Satzgedächtnis“ aus dem SETK 3-5. Mehrheitlich erfolgten die Untersuchungen $\mathrm{zu} \mathrm{t}_{1}, \mathrm{t}_{2}$ und $\mathrm{t}_{3}$ im vertrauten häuslichen Umfeld der Kinder und lediglich zu $\mathrm{t}_{3}$ gab es einen Versuchsleiterwechsel.

Die standardisierten Tests wurden manualgetreu durchgeführt, dokumentiert und ausgewertet.

\subsection{Repräsentativität der Stichprobe}

Um Aussagen darüber machen zu können, inwieweit die sprachlichen Leistungen der Kinder in dieser Untersuchungsstichprobe von $t_{0}$ bis $t_{3}$ mit denen der Normstichprobe übereinstimmen, wurden die Rohwerte aller Kinder in den einzelnen Subskalen zu allen Untersuchungszeitpunkten in T-Werte bzw. Prozentränge umgewandelt. Tabelle 8 stellt die entsprechenden mittleren T-Werte und Prozentränge sowie Minimum, Maximum und Standardabweichung für alle Sprachoutcomevariablen von $\mathrm{t}_{0}$ bis $\mathrm{t}_{3}$ dar. 
Tabelle 8

Deskriptive Statistiken für die erhobenen Sprachmaße von $t_{0}$ bis $t_{3}$

\begin{tabular}{|cccccc|}
\hline & $\boldsymbol{N}$ & Minimum & Maximum & Mittelwert & $\begin{array}{c}\text { Standard- } \\
\text { abweichung }\end{array}$ \\
\hline $\mathbf{t}_{\mathbf{0}}$ & & & & & \\
ELAN PR & 57 & 0 & 100 & 37.53 & 28.67 \\
\hline $\mathbf{t}_{1}$ & & & & & \\
WS PR & 38 & 0 & 97 & 49.18 & 27.54 \\
3 VS_T & 26 & 40 & 64 & 52.73 & 8.14 \\
3 MR_T & 26 & 0 & 72 & 59.73 & 15.24 \\
4 VS_T & 31 & 32 & 74 & 52.09 & 10.51 \\
4 MR_T & 31 & 33 & 66 & 52.19 & 7.44 \\
4 SG_T & 31 & 0 & 67 & 43.00 & 18.36 \\
\hline $\mathbf{t}_{\mathbf{2}}$ & & & & & \\
WS PR & 54 & 2 & 97 & 52.38 & 27.66 \\
VS_T & 54 & 39 & 72 & 55.88 & 7.40 \\
MR_T & 54 & 37 & 72 & 50.75 & 8.04 \\
SG_T & 45 & 31 & 67 & 49.93 & 9.35 \\
\hline $\mathbf{t}_{3}$ & & & & & \\
HSET_VS_T & 53 & 31 & 64 & 48.32 & 6.61 \\
IS_T & 53 & 17 & 59 & 41.53 & 10.4 \\
PS_T & 53 & 35 & 71 & 48.45 & 8.37 \\
HAWIK WS_T & 52 & 38 & 80 & 57.65 & 8.50 \\
\hline
\end{tabular}

Anmerkungen. $N=$ Stichprobengröße, $\mathrm{t}_{0}=$ Messzeitpunkt 2001, $\mathrm{t}_{1,2,3}=1$., 2. und 3. Messzeitpunkt, ELAN PR = Prozentrang im Elternfragebogen zur Wortschatzentwicklung im frühen Kindesalter (ELAN), WS PR = Prozentrang im Aktiven Wortschatztest für 3-6-jährige Kinder, 3 VS_T = T-Wert im Untertest „Verstehen von Sätzen“ aus dem Sprachentwicklungstest für drei- bis fünfjährige Kinder (SETK 3-5) für die dreijährigen Kinder, 3 MR_T = T-Wert im Untertest „Morphologische Regelbildung“ (SETK 3-5) für die dreijährigen Kinder, 4 VS_T = T-Wert im Untertest „Verstehen von Sätzen“ (SETK 3-5) für die vierjährigen Kinder, 4 MR_T = TWert im Untertest „Morphologische Regelbildung“ (SETK 3-5) für die vierjährigen Kinder, 4 SG_T = T-Wert im Untertest „Satzgedächtnis“ (SETK 3-5) für die vierjährigen Kinder, VS_T = T-Wert im Untertest „Verstehen von Sätzen“ (SETK 3-5), MR_T = T-Wert im Untertest „Morphologische Regelbildung“ (SETK 3-5), SG_T = T-Wert im Untertest „Satzgedächtnis“ (SETK 3-5), HSET_VS_T = T-Wert im Untertest „Verstehen grammatischer Strukturformen“ aus dem Heidelberger Sprachentwicklungstest (HSET), IS_T = T-Wert im Untertest „Imitation grammatischer Strukturformen“ (HSET), PS_T = T-Wert im Untertest „Plural-SingularBildung“(HSET), HAWIK WS_T = T-Wert im Untertest „Wortschatz“ aus dem Hamburg Wechsler Intelligenztest für Kinder

Tabelle 8 ist zu entnehmen, dass sich alle mittleren T-Werte im Normbereich von 40 bis 60 bewegen (auch unter Berücksichtigung der in T-Werte umgerechneten mittleren




mittlere T-Wert in der Untersuchungsstichprobe wurde mit 59.7 in der Gruppe der dreijährigen Kinder zu t $t_{1}$ im Untertest MR erreicht. Der niedrigste mittlere T-Wert wurde mit $41.53 \mathrm{zu} \mathrm{t}_{3}$ beim Untertest IS erzielt. Da sich zu diesen Messzeitpunkten die anderen mittleren T-Werte bzw. Prozentränge nicht im unteren bzw. oberen Bereich der Norm befanden und der mittlere $\mathrm{T}$-Wert pro Messzeitpunkt $\left(\mathrm{t}_{1}: \mathrm{T}=51.62, \mathrm{t}_{2}: \mathrm{T}=51.89\right.$ und $\left.\mathrm{t}_{3}=48.99\right)$ eine minimale Höhe von 48.99 bzw. maximal Höhe von 51.89 aufwies, ist eher von einer Über- bzw. Unterschätzung der Leistung durch einzelne Untertests zu bestimmten Messzeitpunkten auszugehen. Die Standardabweichungen variierten für alle Subtests des SETK 3-5, des HSET sowie des HAWIK III von 6.61 bis 18.36. Bei optimaler Vergleichbarkeit mit den Normierungsstichproben der Testverfahren sind Standardabweichungen der T-Werte von 10 zu erwarten.

Insgesamt sprechen die Ergebnisse für eine recht gute Repräsentativität der Stichprobe bzgl. der gemessenen Sprachleistungen. 


\section{Entwicklungsstabilitäten von Sprache}

\subsection{Theoretischer Hintergrund}

In der entwicklungspsychologischen Forschung geht es um die Beobachtung, Beschreibung und Erklärung von Veränderung menschlichen Erlebens und Verhaltens. Konstanz versus Veränderung lässt sich auf zwei Arten definieren: mit Bezug auf die Zeit und mit Bezug auf andere Individuen und deren Veränderung. Beim zeitlichen Bezug geht es um den längsschnittlichen Entwicklungsverlauf von Eigenschaften und Verhaltensweisen. Besteht keine Veränderung über die Zeit, wird von Kontinuität in der Entwicklung gesprochen. Bei bestehenden Veränderungen über die Zeit spricht man von Diskontinuität.

Untersucht man die Entwicklung im Vergleich zu anderen Individuen, spricht man von interindividueller bzw. differentieller Stabilität vs. Instabilität.

Stabilität in diesem Sinne bedeutet, dass keine interindividuellen Unterschiede in den intraindividuellen Veränderungen vorliegen. Stabilität bedeutet nicht, dass keine Veränderungen für das Leistungsmerkmal über die Zeit vorliegen. In Bezug auf Stabilität lassen sich absolute und normative Stabilität unterscheiden. Absolute Stabilität liegt vor, wenn sich weder die Gruppe insgesamt noch die individuellen Positionen innerhalb der Gruppe verändern. Am häufigsten empirisch untersucht wird die normative Stabilität (auch Rangordnungs-Stabilität oder Positionsstabilität genannt). Diese bezeichnet die Konstanz der Position eines Individuums innerhalb der Gruppe und wird mittels Korrelation zwischen zwei Messzeitpunkten quantifiziert. Bei einer Korrelation von 1 behielten alle Personen ihre Position in der Gruppe bei. Beträgt die Korrelation 0, so wäre von völlig unterschiedlichen Veränderungen der Individuen über die Zeit auszugehen. Die Werte der normativen Stabilität machen jedoch keine Aussage darüber, ob und in welchem Ausmaß sich die Gruppe im Mittel verändert.

Fenson et al. (1994) betonen die Bedeutung der normativen Stabilität für das grundlegende Verständnis von Kindersprache: „Understanding individual differences factors is central to elucidating the nature and development of child language.“ (zitiert nach Bornstein, Haynes, Painter \& Genevro, 2000, S. 407). Aussagen zur Stabilität von Sprache ermöglichen somit rekursiv Aussagen zu bestehenden Spracherwerbstheorien.

Von praktischer Relevanz sind die Erkenntnisse zur Stabilität von Sprache mit Hinblick auf Vorhersagen späterer Sprachleistungen aus frühen Sprachmaßen. Ist Sprache ein Merkmal von hoher Stabilität, so sind bedeutsame Vorhersagen hoher Effektstärke für späteres Sprachoutcome möglich und aus dem Bestehen von schlechten Sprachleistungen mit 2 Jahren kann z.B. auf das Bestehen schlechter Sprachleistungen im Alter von 4 oder 5 Jahren 
geschlossen werden. In diesem Fall müssten schon im frühen Alter von 2 Jahren bei schlechten Sprachleistungen dringend präventive Interventionsmaßnahmen eingeleitet werden.

In dieser Arbeit ist die normative Stabilität Untersuchungsgegenstand und soll über die Pearson Produkt-Moment-Korrelationen bezüglich der erhobenen Sprachvariablen zwischen den unterschiedlichen Messzeitpunkten geschätzt werden.

Bei allen Ergebnissen von Studien, die sich mit der Vorhersage und Untersuchung von Entwicklung befassen, ist zu berücksichtigen, dass Wechselwirkungen zwischen Personenmerkmalen (wie Disposition und Kompetenzen) und wirksamen Umweltfaktoren (z.B. familiäre Situation und Schulunterricht) bestehen. Somit bleibt unklar in welchem Ausmaß individuelle Variationen ein Attribut des Kindes repräsentieren oder auf stabile (bzw. instabile) Umweltbedingungen zurückzuführen sind. Dieses gilt auch für die Sprachentwicklung. Bei Feststellung einer stabilen sprachlichen Entwicklung kann also konstatiert werden, dass die Kinder den Rang innerhalb der Bezugsgruppe beibehalten. Solch ein Ergebnis ist einerseits beschreibbar als Ausdruck stabiler endogener Charakteristika der Kinder. Zum anderen ist aber gerade die Sprachentwicklung ein erfahrungsabhängiges Phänomen. Stabilität der sprachlichen Entwicklung ließe sich somit auch beschreiben als Ausdruck einer stabilen Sprachumgebung.

Des Weiteren wird in der aktuellen Forschungsliteratur zwischen heterotyper und homotyper Stabilität (im angloamerikanischen Sprachraum „heterotypic“ versus „complete stability“) unterschieden. Homotype Stabilität meint die Stabilität in den Leistungen bezogen auf gleiche Konstrukte bzw. Untersuchungsaspekte über die Zeit (z.B. die Korrelationen von Wortschatzdaten $\mathrm{zu} \mathrm{t}_{0}$ mit den Wortschatzdaten $\mathrm{zu}_{1}$ ). Mit heterotyper Stabilität bezeichnet man die Stabilität der erhobenen Leistungen in unterschiedlichen Konstrukten bzw. Untersuchungsaspekten (z.B. die Korrelationen von Wortschatzdaten $\mathrm{zu} \mathrm{t}_{0}$ mit den Satzgedächtnisleistungen $\mathrm{zu} \mathrm{t}_{1}$ ). Übereinstimmend sind die dokumentierten Werte für homotype Stabilität höher als die für heterotype Stabilität (z.B. Bornstein, Hahn \& Haynes, 2004). In dieser Arbeit soll es um die Ermittlung und Darstellung der homotypen Stabilität von Sprachentwicklung gehen.

In einer Vielzahl von Untersuchungen werden die Ergebnisse zu den Stabilitäten von Sprachentwicklung nach der Höhe des Zeitintervalls betrachtet, welches zwischen den verglichenen Messzeitpunkten liegt. Uneinigkeit herrscht hierbei in der Verwendung der Begriffe „long-term-stability“ und „short-term-stability“. So bezeichnen Bornstein et al., (2000) das Intervall von einer Woche ebenso als „,short-term-stability“ wie die Zeitspanne von 
sieben Monaten (Bornstein et al., 2004). In dieser Arbeit wird auf die Verwendung dieser Begriffe verzichtet, zum einen aufgrund der fehlenden Übereinstimmung bzw. Konvention in der Definition der Begriffe und zum anderen aufgrund des damit verbundenen Informationsverlustes. Alternativ wird das jeweilige Bezugs-Zeitintervall (z.B. von $\mathrm{t}_{0} \mathrm{zu} \mathrm{t}_{1}=$ 26 Monate oder von $\mathrm{t}_{1} \mathrm{zu} \mathrm{t}_{2}=12$ Monate) explizit benannt.

Uneinigkeit besteht weiterhin in der Beurteilung der Höhe der Stabilitäten. So erweckt es den Anschein, dass Begrifflichkeiten wie „schwach“, „moderat“, ,ausgezeichnet“ und „stark“ (bzw. in der angloamerikanischen Literatur „small“, „moderate“, „large“, „substantial“, „excellent“ und „strong“) eher nach persönlichem Maßstab der einzelnen Autoren vergeben werden denn nach bestimmten allgemein akzeptierten Konventionen bzw. diese Konventionen nicht expliziert werden. So werden innerhalb einer Veröffentlichung Korrelationen von .23 - .34 als ,niedrige bis moderate“ Stabilitäten bezeichnet, gleichzeitig Korrelationen von .33-.67 mit dem Begriff „mittlere Stabilitäten“ belegt und eine Korrelation von .63 als ,relativ hoch“ bewertet (Krajewski, Kron \& Schneider, 2004). Eine Ausnahme stellen Bornstein et al. (2004) dar, die sich in ihrem Vorgehen an die Konventionen von Cohen (1988) anlehnen und Güte von Stabilitäten in Bezug auf die entsprechenden Effektgrößen bewerten. Demnach verfügt eine Korrelation von .10 über eine kleine Effektgröße (,small effect“"), eine Korrelation von .30 über eine mittlere Effektgröße (,,medium effect“") und eine Korrelation von .50 über eine große Effektgröße (,large effect“"). Für das weitere Vorgehen in dieser Arbeit wird auch in diesem Punkt von der Explizierung der Höhe der Korrelationen in Form von nicht allgemeingültigen Begriffen zu Gunsten des Informationsgehaltes der Ergebnisse abgesehen und es kommen die Konventionen von Cohen (1988) zur Anwendung.

Ein weiterer Aspekt in der Betrachtung von Entwicklungsstabilitäten ist die phylogenetische (stammesgeschichtliche) versus ontogenetische (die Einzelentwicklung betreffende) Perspektive. Untersuchungsgegenstand dieser, wie der meisten Arbeiten zum Thema Entwicklungsstabilitäten, ist die ontogenetische Betrachtungsweise. Erwähnt sei aber an dieser Stelle, dass sich z.B. im Gegensatz zur Intelligenzentwicklung Hinweise für die Stabilität der phylogenetischen Entwicklung von Sprache finden lassen. So ergaben die aktuellen Untersuchungen von Rohwerteverteilungen des Heidelberger Sprachentwicklungstest (HSET) annähernde Entsprechungen mit den Rohwerteverteilungen des HSET von 1975. Dieses deutet darauf hin, dass kein Zuwachs z.B. bezüglich der grammatischen Leistungsentwicklung in den letzten 30 Jahren erfolgt ist (H. Schöler, persönliche Mitteilung 4.3.2007). 
Die Mehrheit der Studien zur Entwicklungsstabilität befasst sich mit der kognitiven Entwicklung (Spiel, Schabmann \& Glück, in Druck). Für kognitive Kompetenzen gilt eine relativ frühe Stabilisierung individueller Unterschiede (Roberts \& DelVecchio, 2000). Studien mit standardisierten Testverfahren demonstrieren eine geringe Stabilität für die ersten beiden Lebensjahre, so dass frühestens ab einem Alter von 18 bis 24 Monaten Vorhersagen späterer Intelligenztestwerte mit entsprechenden Verfahren möglich sind. Die Stabilität steigt dann bis zum Alter von etwa 50 Jahren an. Die Stabilität affektiver Variablen und sozialer Verhaltensweisen ist niedriger als jene von Leistungsmerkmalen (Spiel et al., in Druck). Eine Ausnahme stellt hier die relativ frühe Stabilität von Aufmerksamkeitsleistungen insbesondere in Bezug auf spätere Schulleistungen dar (Ruff, Lawson, Parrinello \& Weissberg, 1990). Mehrheitlich wird die Stabilität von Schulleistungen (in Abhängigkeit von der Form der Messung) als relativ hoch eingeschätzt (z.B. Weinert \& Helmke, 1993).

Allgemein gilt, dass die Höhe der Stabilität (gemessen mittels Korrelation) abhängt von dem Zeitintervall zwischen den Messzeitpunkten (je kürzer der Zeitabstand, desto höher die Stabilität) sowie der Reliabilität der Messung. Hierbei kann z.B. die Verwendung unterschiedlicher Erhebungsinstrumente für eine Variable über die Zeit zur Verringerung der Korrelationen und somit der ermittelten Stabilitäten führen. Die Stichprobengröße entscheidet über die Güte der Schätzung der Stabilität. Je größer die Stichprobe, desto besser ist die Schätzung der Stabilität.

Es gibt eine Vielzahl an Veröffentlichungen zur Stabilität von Sprachentwicklungsstörungen, die je nach Stichprobe, verwendeten Untersuchungsverfahren und insbesondere der verwendeten Störungsdefinition $\mathrm{zu}$ unterschiedlichen Ergebnissen kommen. So berichtet Silva (1980) über geringe Stabilität von „developmental language delay“ in einer Stichprobe von 937 neuseeländischen Kindern im Alter von 3 Jahren (Nachuntersuchung mit 5 Jahren). Tomblin, Zhang, Buckwalter und O`Brien (2003) stellen fest, dass in einer Stichprobe von 196 Kindergartenkindern mit „primary language delay“ die Schwächen bezüglich der Sprache bis in die vierte Grundschulklasse erhalten blieben, wenn die Bedingungen für Regression zur Mitte kontrolliert wurden.

Je nach Untersuchungsalter und Zeitintervallen zwischen den Messzeitpunkten lassen sich dem aktuellen Forschungsstand unterschiedliche Angaben zur Stabilität von Sprache bezogen auf den regelrechten Spracherwerb entnehmen. Allgemein deutet die Auswertung der Forschungsliteratur zur Stabilität von Sprache (homotyper und heterotyper) auf signifikante Stabilitäten (relativ hoher Effektstärken) über die Zeitspanne von wenigen Wochen bis zu 2 Jahren hin (z.B. Pine, Lieven \& Rowland, 1996 und Sparrow, Balla \& Cicchetti, 1984). 
In einer aktuellen Untersuchung zur Entwicklung drei- bis vierjähriger Kinder konnte sich der Bereich Sprache im Vergleich zu den Bereichen Theory of Mind und Arbeitsgedächtnis als stabilstes Merkmal bzgl. der interindividuellen Differenzen beweisen (Lock1, Schwarz \& Schneider, 2004).

Bornstein, Putnick und De Houwer (2006) widmen sich der Untersuchung des frühen Wortschatzes im Zeitfenster von 13 bis 20 Monaten. Da sich das Alter von 1 bis 2 Jahren durch eine hohe Variabilität des Wortschatzes auszeichnet (Fenson et al., 1994), ist es von besonderem Interesse bezogen auf Stabilitätsuntersuchungen. In einer Untersuchung mit einer niederländischen Adaptation der Mac Arthur Communicative Development Inventories (CDI) (Fenson et al., 1993) an 29 Kindern konnten übereinstimmend für alle Informanden signifikante moderate bis hohe Stabilitäten für Sprachverstehen $(r=.56-.81)$ und -produktion ( $r=.31-.48)$ bei signifikanten Leistungszuwächsen in den durchschnittlichen Verstehens- und Produktionsleistungen gefunden werden. Die Stabilitäten des rezeptiven Wortschatzes waren hierbei tendenziell höher als die des aktiven Wortschatzes (keine signifikanten Korrelationsdifferenzen).

Eine Studie mit 33 zweijährigen Kindern unter Verwendung von Spontansprachanalysen in familiärem Setting versus Laborbedingung (Bornstein et al., 2000) erbrachte durchschnittliche Stabilitätswerte für die Zeitspanne von einer Woche bezogen auf die frühen Sprachmaße „MLU“, „total utterances“ und ,word roots“ von .67 (range: $r=.39$ - .84). Hierbei meint „total utterances“ die Anzahl aller Äußerungen in einem bestimmten Zeitintervall und mit „word roots“ ist die Anzahl aller Worte unterschiedlichen Wortstamms beschrieben. Die Autoren schlussfolgern das Bestehen einer moderaten bis hohen „shortterm-stability“ unabhängig von der Untersuchungssituation.

Abschließend soll auf zwei Studien näher eingegangen werden, die zur Einordnung der in dieser Arbeit berichteten Ergebnisse zu Entwicklungsstabilitäten von Sprache gut geeignet sind. Denn zum einen werden ähnliche Zeitfenster untersucht und zum anderen bieten die (zum Teil) deutlich größeren Untersuchungsstichproben die Möglichkeit der Beurteilung zur Repräsentativität der Untersuchungsstichprobe dieses Dissertationsprojektes mit Hinblick auf den Untersuchungsaspekt Entwicklungsstabilitäten.

Bornstein et al. (2004) berichten über vier Längsschnittstudien zur Untersuchung der Stabilität von Sprache mit besonderem Hinblick auf die Variable Geschlecht. Die untersuchte Altersspanne belief sich auf 1;1 bis 6;10 Jahre. Die Stichprobengrößen variierten von 26 bis 185 und als Erhebungsverfahren kamen Parent Report, standardisiertes Testen sowie Spontansprachanalysen zum Einsatz. Als Schätzungen der Stabilitäten wurden Korrelationen 
verwendet. Es wurden sowohl Werte für die homotypen als auch heterotypen Stabilitäten dokumentiert, wobei letztere aufgrund der Verwendung unterschiedlicher Erhebungsverfahren als konservative Schätzungen der „wahren“ Stabilitäten zu werten sind. Die Untersuchungen ergaben, dass die Stabilitäten (homogene und heterotype) für Jungen und Mädchen im Alter von 1;1 bis 6;10 Jahren sich nicht signifikant unterscheiden. Darüber hinaus waren die ermittelten Stabilitäten unabhängig von dem Faktor „Bildung der Mutter“. Schlussfolgernd wurde nicht nur aufgrund der kleinen Stichprobe in dieser Arbeit auf eine differenzierte Betrachtung der Stabilitäten bezogen auf den Faktor Geschlecht und Bildung der Eltern verzichtet. Studie $1(n=26)$ berichtet über mittlere Stabilitäten von 2;0 zu 3;1 Jahren von .63 und von 2;0 zu 4;0 Jahren von .29. In Studie $2(n=184)$ wurden für das Alter von 1;8 bis 4;0 Stabilitäten von .05 bis .47 errechnet (mittlere Stabilität: $M_{\mathrm{r}}=.29$ ). Studie 3 untersuchte an 34 Kindern $\mathrm{zu}$ den Messzeitpunkten 1;1 Jahre, 1;8 Jahre und 5;10 Jahre die Entwicklungsstabilitäten von Sprache. Für die Altersspanne von 7 Monaten (1;1 bis 1;8 Jahre) ergab sich eine mittlere Stabilität von .40. Für die Spanne von 1;8 bis 5;10 Jahren ließ sich lediglich für das standardisierte Testen (nicht für Parent Report) eine signifikante mittlere Korrelation von .40 finden. Keine signifikanten Korrelationen für die frühen Parent-ReportMaße ließen sich für die Altersspanne von 1;1 bis 5;10 Jahren dokumentieren. In Studie 4 ( $n$ = 85) wurden die Ergebnisse zu den Messzeitpunkten 4;6 und 6;10 Jahren in Beziehung gesetzt. Es ergaben sich Stabilitäten von .25 bis .55. Zusammenfassend berichten die Autoren von moderaten bis hohen normativen Stabilitäten der spezifischen und allgemeinen Sprachentwicklung für die Altersspanne von 1 bis 7 Jahren und betonen, dass neben homotyper Stabilität auch heterotype Stabilität in altersangemessenen unterschiedlichen Spracherhebungsverfahren zu zugrunde liegenden Sprachprozessen nachgewiesen werden konnte.

Weber, Marx und Schneider (2005) untersuchten an einer Stichprobe von 111 Kindern über die Zeitspanne von 4 bis $7_{3 / 4}$ Jahren u. a. die Stabilität von Wortschatz und Grammatik. Hierbei kamen als Testverfahren ebenfalls der SETK 3-5 sowie der HSET zum Einsatz. Zu einem Grammatikwert wurden die Untertests VS, MR und SG aus dem SETK 3-5 sowie die Untertests VS, PS und IS aus dem HSET zusammengefasst. Der Wortschatz wurde mit dem Hannover-Wechsler-Intelligenztest für das Vorschulalter (HAWIVA; Eggert \& Schuck, 1975) erfasst. Insgesamt beurteilen die Autoren die Stabilitäten der einzelnen Sprachkomponenten als „recht stabil“. Für den Grammatikwert beliefen sich die Korrelationen von 4 bis $73 / 4$ Jahren auf Werte von .56 bis .73. Für den Wortschatz ließen sich Korrelationen von .33 bis .51 errechnen. 
Zwei Forschungsfragen ergeben sich aus der Kenntnis des aktuellen Forschungsstandes in Bezug auf das vorliegende Untersuchungsdesign:

1. Sind alle erhobenen Sprachmaße über die komplette Zeitspanne (von bis zu 6 Jahren) und in den unterschiedlichen Altersabschnitten (z.B. von 22 Monaten zu 4;0 Jahren bzw. von 5;0 Jahren zu 7;10 Jahren) stabil oder gibt es Ausnahmen? Das heißt, gibt es Phasen im Entwicklungsverlauf, in denen Sprache kein stabiles Merkmal ist?

2. Wie ausgeprägt ist die Stabilität der Sprachmaße über die Zeit und in den unterschiedlichen Altersabschnitten?

Die vielfältigen Aussagen zum Vorliegen von bedeutsamer Stabilität von Sprache in der Kindheit sowie der Tatbestand, dass es in der vorliegenden Untersuchung um die Ermittlung homotyper Stabilitäten geht, führen zu folgenden psychologischen Hypothesen:

PH 1: Für ein Kind mit relativ guten Wortschatzleistungen (Rangposition innerhalb einer Bezugsgruppe) in der frühen Kindheit werden auch relativ gute Wortschatzleistungen im Alter von 4;0, 5;0 und 7;10 Jahren erwartet. Die gleiche Erwartung gilt für die Zeitspanne von $4 ; 0$ zu 5;0 Jahren und von 4;0 zu 7;10 Jahren sowie von 5;0 zu 7;10 Jahren.

PH 2: Für ein Kind mit relativ guten rezeptiven grammatischen Leistungen (Rangposition innerhalb einer Bezugsgruppe) im Alter von 4;0 Jahren werden ebenfalls relativ gute rezeptive grammatische Leistungen im Alter von 5;0 und 7;10 Jahren erwartet. Die gleiche Erwartung gilt für die Zeitspanne von 5;0 Jahren zu 7;10Jahren.

PH 3: Für ein Kind mit relativ guten expressiven grammatischen Fähigkeiten (Rangposition innerhalb einer Bezugsgruppe) in der frühen Kindheit werden auch relativ gute expressive grammatische Fähigkeiten im Alter von 4;0, 5;0 und 7;10 Jahren erwartet. Die gleiche Erwartung gilt für die Zeitspanne von 4;0 zu 5;0 Jahren und von 4;0 zu 7;10 Jahren sowie von 5;0 zu 7;10 Jahren.

PH 4: Für ein Kind mit relativ guten Satzgedächtnisleistungen (Rangposition innerhalb einer Bezugsgruppe) im Alter von 4;0 Jahren werden auch relativ gute Satzgedächtnisfähigkeiten im Alter von 5;0 und 7;10 Jahren erwartet. Die gleiche Erwartung gilt für die Zeitspanne von 5;0 Jahren zu 7;10 Jahren. 


\subsection{Ergebnisse}

\subsubsection{Kohärenz der Sprachdaten}

Zunächst einmal soll der Frage nachgegangen werden, ob die in dieser Studie mit den ausgewählten Untersuchungsverfahren erhobenen unterschiedlichen Aspekte von Sprache zu allen Messzeitpunkten in einem signifikanten Zusammenhang miteinander stehen. Tun sie dieses, dann kann von einem kohärenten Untersuchungsgegenstand „Sprache“ ausgegangen werden und die Ergebnisse im weiteren Verlauf entsprechend interpretiert werden (z.B. als Entwicklungsstabilitäten von „Sprache“). $\mathrm{Zu}$ erwarten ist die Abnahme der Zusammenhangsstärken über die Zeit. In anderen Untersuchungen (z.B. Lockl \& Schneider, 2007) werden die Ergebnisse in den verschiedenen sprachlichen Untertests über die Zeit auf Kohärenz untersucht, um dann neben den Ergebnissen der Faktorenanalyse über ein Argument für die Zusammenfassung der Daten zu einem gemeinsamen Faktor „Sprache“ zu verfügen. Ziel dieser Arbeit ist es jedoch den Informationsgehalt der einzelnen Untertests zu erhalten, um z.B. Aussagen zur Stabilität des Wortschatzes und zur Stabilität des Satzgedächtnisses machen zu können.

Für alle Messzeitpunkte wurden die Pearson-Produkt-Moment-Korrelationen der erhobenen Sprachmaße berechnet (einseitige Testung, $p<0.05$ ).

$\mathrm{Zu} \mathrm{t}_{0}$ gingen der Prozentrang im ELAN als Wortschatzmaß und die MLU als Maß der frühen expressiven und rezeptiven grammatischen Fähigkeiten in die Berechnung ein. Die Summe an Pluralwörtern im ELAN wurde als zusätzliches Maß für die expressiven grammatischen Fähigkeiten festgelegt, da sie mit der Operationalisierung dieses Sprachaspektes zu späteren Messzeitpunkten (Morphologische Regelbildung) gut vergleichbar ist.

$\mathrm{Zu} \mathrm{t}_{1}$ wurden für die dreijährigen Kinder die Rohwerte im AWST 3-6 (Wortschatz), die Rohwerte im Untertest „Verstehen von Sätzen“ (SETK 3-5; rezeptive grammatische Fähigkeiten) sowie die Rohwerte im Untertest „Morphologische Regelbildung“ (SETK 3-5; expressive grammatische Fähigkeiten) korreliert. Für die vierjährigen Kinder wurden zusätzlich die Rohwerte im Untertest „Satzgedächtnis“ (SETK 3-5; Satzgedächtnisleistungen) in die Korrelationsanalysen einbezogen.

$\mathrm{Zu} \mathrm{t}_{2}$ wurden die Rohwerte im AWST 3-6 (Wortschatz), die Rohwerte im Untertest „Verstehen von Sätzen“ (SETK 3-5; rezeptive grammatische Fähigkeiten) sowie die Rohwerte im Untertest „Morphologische Regelbildung“ (SETK 3-5; expressive grammatische Fähigkeiten) und die Rohwerte im Untertest „Satzgedächtnis“ (SETK 3-5; Satzgedächtnisfertigkeiten) korreliert. 
$\mathrm{Zu} \mathrm{t}_{3}$ gingen in die Korrelationsanalysen jeweils die Rohwerte der im Folgenden genannten Subtests ein: für den Wortschatz der Untertest „Wortschatz“ (HAWIK III), für die rezeptiven grammatischen Fähigkeiten der Untertest „Verstehen grammatischer Strukturformen“ (HSET), für die expressiven grammatischen Fähigkeiten der Untertest „Plural-Singular-Bildung“ (HSET) und für das Satzgedächtnis der Untertest „Imitation grammatischer Strukturformen“ (HSET).

Die Abbildungen 1 bis 5 veranschaulichen die Ergebnisse der Korrelationsanalysen für die Untersuchungszeitpunkte $\mathrm{t}_{0}$ bis $\mathrm{t}_{3}{ }^{1}$

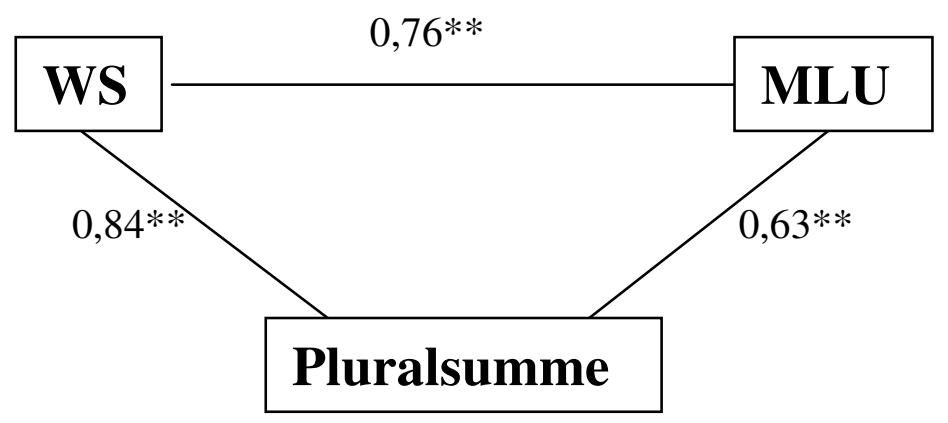

Anmerkungen. $*=$ Irrtumswahrscheinlichkeit $p<0.05$, ** $=$ Irrtumswahrscheinlichkeit $p<0.01$, WS $=$ Prozentrang im ELAN, MLU = Mean Length of Utterances, Pluralsumme = Summe aller Pluralwörter im ELAN

Abbildung 1

Zusammenhänge von Wortschatzleistungen (WS) und expressiven grammatischen Fähigkeiten (MLU und Pluralsumme) zu to

\footnotetext{
${ }^{1}$ Bezogen auf die Mehrheit der Variablen konnte die Normalverteilungsannahme der Rohdaten nicht bestätigt werden. Die Verwendung der entsprechenden z-standardisierten Werte in den Korrelationsanalysen führte jedoch zu keinen bedeutsamen Unterschieden in den Ergebnissen. Auch für die weiteren Variablen und Berechnungen in dieser Arbeit ergaben sich bei Verwendung der z-transformierten Werte keine substanziellen Veränderungen in den Ergebnissen. Folglich werden mit Ausnahme des frühen Wortschatzes die Rohwerte der jeweiligen Untertests bei den statistischen Analysen Anwendung finden.
} 


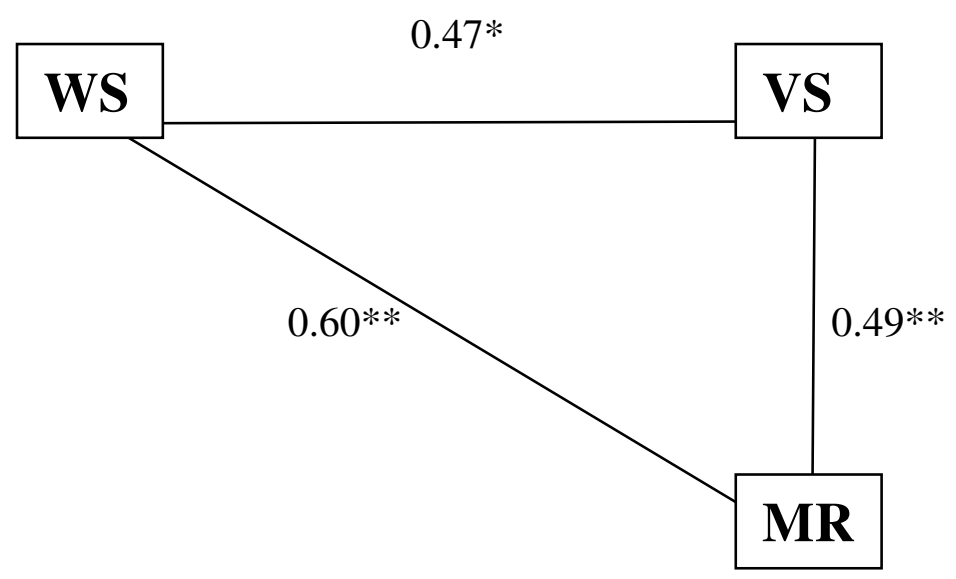

Anmerkungen. $*$ Irrtumswahrscheinlichkeit $p<.05, * *=$ Irrtumswahrscheinlichkeit $p<.01, \mathrm{WS}=$ Aktiver Wortschatztest für drei- bis fünfjährige Kinder, VS = Untertest „Verstehen von Sätzen“ aus dem Sprachentwicklungstest für drei- bis fünfjährige Kinder, MR = Untertest „Morphologische Regelbildung“ aus dem Sprachentwicklungstest für drei- bis fünfjährige Kinder

Abbildung 2

Zusammenhänge von Wortschatzleistungen, expressiven grammatischen Fähigkeiten (MR) und rezeptiven grammatischen Fähigkeiten (VS) für die dreijährigen Kinder zu $t_{1}$

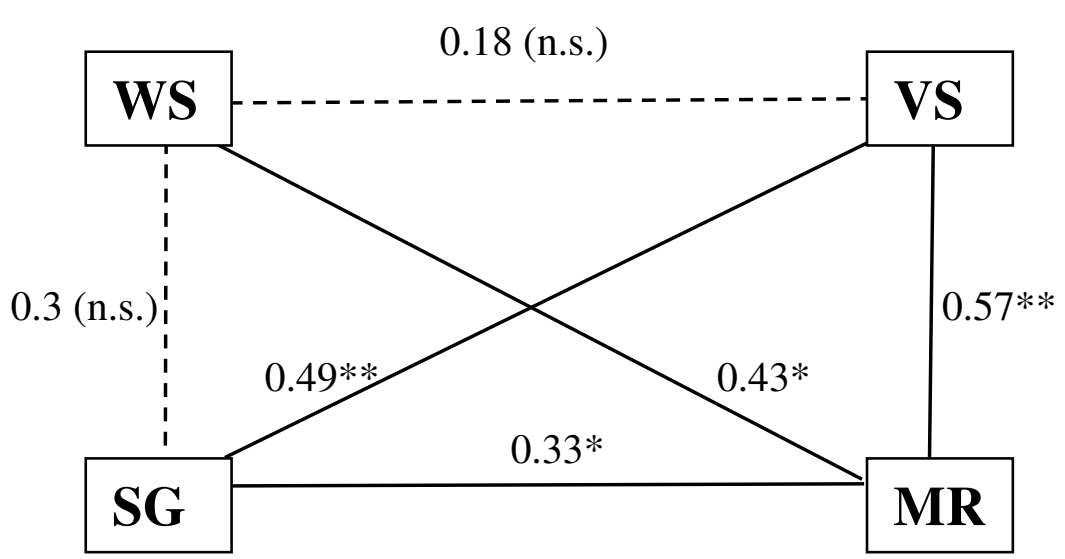

Anmerkungen. ${ }^{*}=$ Irrtumswahrscheinlichkeit $p<0.05, * *=$ Irrtumswahrscheinlichkeit $p<0.01$, n.s. $=$ nicht signifikant, WS = Aktiver Wortschatztest für drei- bis fünfjährige Kinder, VS = Untertest „Verstehen von Sätzen“ aus dem Sprachentwicklungstest für drei- bis fünfjährige Kinder, SG = Untertest „Satzgedächtnis“ aus dem Sprachentwicklungstest für drei- bis fünfjährige Kinder, MR = Untertest „Morphologische Regelbildung“ aus dem Sprachentwicklungstest für drei- bis fünfjährige Kinder

Abbildung 3

Zusammenhänge von Wortschatzleistungen, expressiven grammatischen Fähigkeiten (MR), rezeptiven grammatischen Fähigkeiten (VS) und Satzgedächtnisfertigkeit (SG) für die vierjährigen Kinder zu $t_{1}$ 


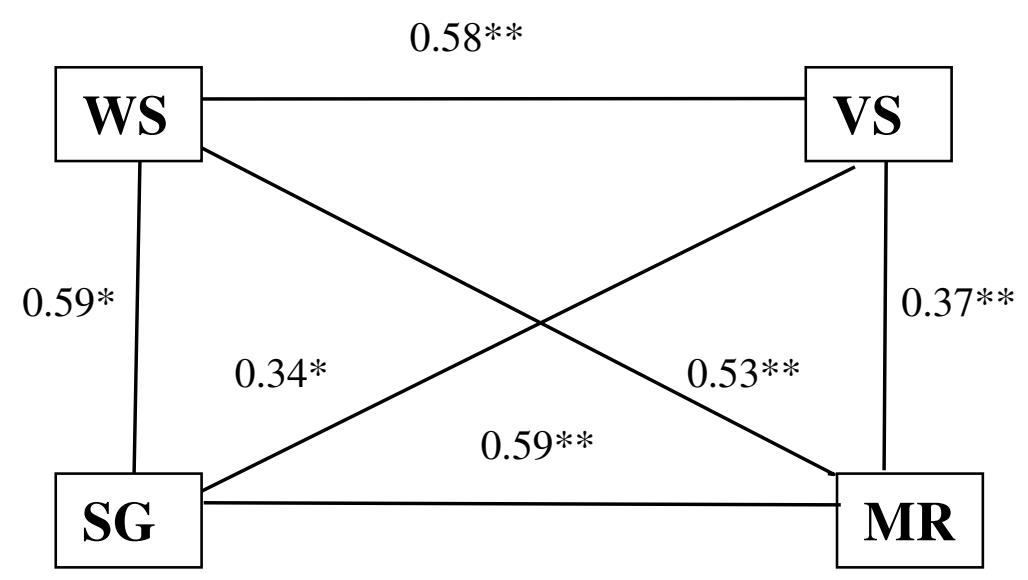

Anmerkungen. $*=$ Irrtumswahrscheinlichkeit $\mathrm{p}<0.05, * *=$ Irrtumswahrscheinlichkeit $p<0.01$, n.s. $=$ nicht signifikant, WS = Aktiver Wortschatztest für drei- bis fünfjährige Kinder, VS = Untertest „Verstehen von Sätzen“ aus dem Sprachentwicklungstest für drei- bis fünfjährige Kinder, SG = Untertest „Satzgedächtnis“ aus dem Sprachentwicklungstest für drei- bis fünfjährige Kinder, MR = Untertest „Morphologische Regelbildung“ aus dem Sprachentwicklungstest für drei- bis fünfjährige Kinder

Abbildung 4

Zusammenhänge von Wortschatzleistungen (WS), expressiven grammatischen Fähigkeiten $(M R)$, rezeptiven grammatischen Fähigkeiten (VS) und Satzgedächtnisleistungen (SG) zu $t_{2}$

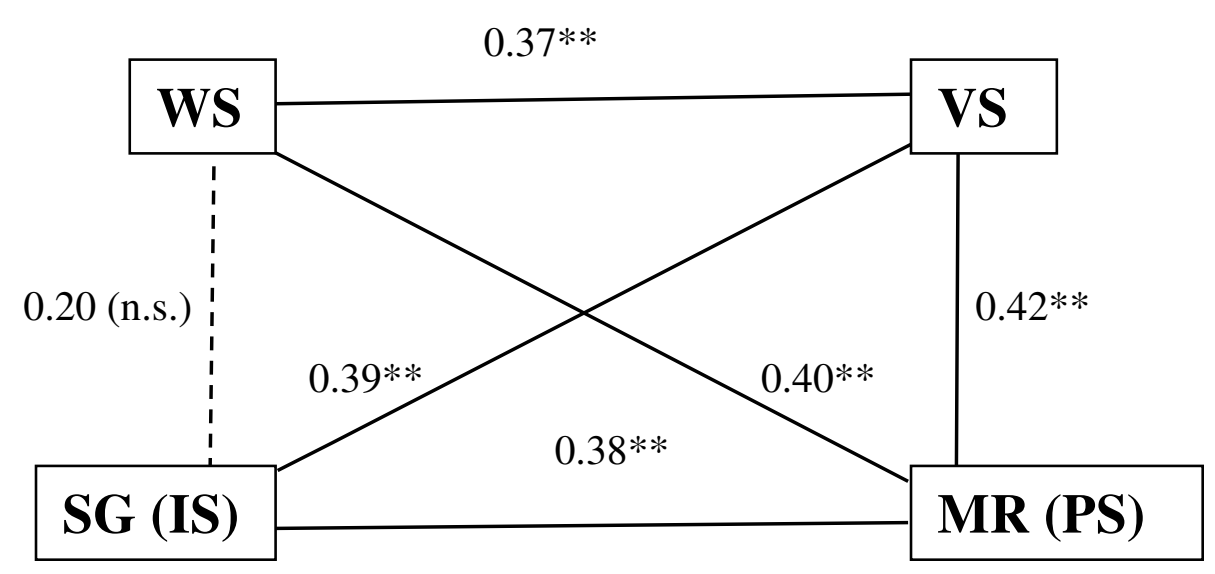

Anmerkungen. ${ }^{*}=$ Irrtumswahrscheinlichkeit $p<0.05, * *=$ Irrtumswahrscheinlichkeit $p<0.01$, n.s. $=$ nicht signifikant, WS = Untertest „Wortschatz“ aus dem Hamburg Wechsler Intelligenztest für Kinder, VS = Untertest „Verstehen grammatischer Strukturformen“ im Heidelberger Sprachentwicklungstest, SG (IS) = Untertest „Imitation grammatischer Strukturformen“ im Heidelberger Sprachentwicklungstest, MR (PS) = Untertest „Plural-Singular- Bildung“ im Heidelberger Sprachentwicklungstest

Abbildung 5

Zusammenhänge von Wortschatzleistungen (WS), expressiven grammatischen Fähigkeiten $(M R)$, rezeptiven grammatischen Fähigkeiten (VS) und Satzgedächtnisleistungen (SG) zu t3 
Im Hinblick auf die Kohärenz der Aufgaben zur Sprachentwicklung konnten zu allen vier Untersuchungszeitpunkten mit nur drei Ausnahmen (Wortschatz und rezeptive grammatische Fähigkeiten der vierjährigen Kinder zu $\mathrm{t}_{1}$; Wortschatz und Satzgedächtnis der vierjährigen Kinder $\mathrm{zu} \mathrm{t}_{1}$ sowie Wortschatz und Satzgedächtnis $\mathrm{zu} \mathrm{t}_{3}$ ) signifikante Zusammenhänge zwischen den Ergebnissen in den einzelnen Subtests bzw. Testteilen festgestellt werden. Diese Interkorrelationen beliefen sich zu t $\mathrm{t}_{0}$ auf .64 bis .84 und zu $\mathrm{t}_{3}$ auf .38 bis .43 .

Zum einen wird deutlich, dass die in dieser Studie gewählten Operationalisierungen der untersuchten Sprachaspekte zur Erfassung von Sprache als kohärentem Konstrukt führen und dass die Betrachtung der Satzgedächtnisfertigkeiten als sprachliche Leistungen gerechtfertigt $\mathrm{zu}$ sein scheint. Zum anderen sprechen die Ergebnisse für die Repräsentativität der Untersuchungsstichprobe, denn Schneider, Lockl und Fernandez (2005) berichten in ihrer Untersuchung an einer größeren Stichprobe von $n=176$ im Alter von 3;4 und 3;10 Jahren über vergleichbare Interkorrelationen bezüglich der SETK 3-5 Subtests, die wiederum mit denen der Normierungsstichprobe des SETK 3-5 gut übereinstimmen. Die Korrelationen zwischen Wortschatz (hier gemessen mit dem Untertest Wortschatz aus dem HAWIVA) und den Untertests des SETK 3-5 waren ebenfalls geringer als die Korrelationen der SETK 3-5 Subtests untereinander. Für die Stichprobe der vierjährigen Kinder $\mathrm{zu} \mathrm{t}_{1}$ ließen sich in vorliegender Untersuchung (im Gegensatz $\mathrm{zu}$ den dreijährigen Kindern $\mathrm{zu} \mathrm{t}_{1}$ ) keine signifikanten Zusammenhänge zwischen Wortschatz und Verstehen von Sätzen sowie Satzgedächtnis errechnen, was durch die geringe Stichprobengröße ursächlich bedingt sein könnte.

Wie von Lockl, Schwarz und Schneider (2004) berichtet, nehmen die Korrelationen zwischen den sprachlichen Subtests über das Alter ab. Dieses gilt auch für vorliegende Stichprobe, was als weiterer Hinweis für die Repräsentativität der Stichprobe gewertet werden kann.

\subsubsection{Leistungszuwachs}

Kinder erwerben durch das Zusammenspiel innerer und äußerer Faktoren innerhalb von wenigen Jahren das hoch komplexe Regelsystem der menschlichen Sprache (Grimm \& Weinert, 2005). Das Merkmal Sprache gilt also insbesondere für die untersuchte Zeitspanne von frühem Kindesalter bis Grundschulalter als diskontinuierliches Merkmal. Folglich sind signifikante Leistungszuwächse der Gesamtstichprobe für die sprachlichen Aspekte Wortschatz, rezeptive und expressive grammatische Fertigkeiten sowie Satzgedächtnis zu erwarten. Zur weiteren Absicherung der Repräsentativität der Stichprobe hinsichtlich des 
Untersuchungsaspektes „Stabilität von Sprache“ soll geprüft werden, ob die Stichprobe sich auch bezogen auf den Aspekt „Kontinuität vs. Diskontinuität“ erwartungskonform verhält.

Da für die großen Zeitabstände zwischen $t_{0}$ und $t_{1}$ (26 Monate) sowie $t_{2}$ und $t_{3}$ (34 Monate) sicher signifikante Leistungsverbesserungen bzgl. der Sprachentwicklung zu erwarten sind und zudem die Verwendung unterschiedlicher Erhebungsverfahren die Vergleichbarkeit der Ergebnisse einschränkt, wurden lediglich für die Zeitspanne von $\mathrm{t}_{1} \mathrm{zu}_{2}$ (12 Monate) signifikante Leistungszuwächse in allen Subtests zur sprachlichen Entwicklung (SETK 3-5 und AWST 3-6) mittels t-Tests untersucht und die Ergebnisse in Tabelle 9 veranschaulicht.

Tabelle 9

Testergebnisse für die Prüfung der Mittelwertsdifferenzen über alle Sprachmaße von $t_{1} z u t_{2}$

\begin{tabular}{|cccccc|}
\hline Sprachmaße & $\boldsymbol{M}_{\mathbf{1}}(\mathbf{S D})$ & $\boldsymbol{M}_{\mathbf{2}}(\mathbf{S D})$ & $\boldsymbol{d} \boldsymbol{f}$ & $\boldsymbol{t}$ & $\boldsymbol{p}$ \\
\hline Wortschatz & $40.67(1.57)$ & $57.39(1.41)$ & 35 & 21.80 & 0.00 \\
$\begin{array}{c}\text { Verstehen von } \\
\quad \text { Sätzen }\end{array}$ & $9.10(3.13)$ & $12.30(1.39)$ & 29 & 7.26 & 0.00 \\
$\begin{array}{c}\text { Morphologische } \\
\text { Regelbildung }\end{array}$ & $20.10(5.65)$ & $23.50(4.24)$ & 29 & 3.78 & 0.00 \\
Satzgedächtnis & $59.06(31.66)$ & $84.11(20.30)$ & 25 & 4.99 & 0.00 \\
\hline
\end{tabular}

Anmerkungen. $M=$ Mittelwert, $S D=$ Standardabweichung, $d f=$ Freiheitsgrade, $t=\mathrm{T}$-Wert, $p=$

Fehlerwahrscheinlichkeit

Für alle untersuchten Sprachmaße lagen erwartungsgemäß signifikante Leistungszuwächse von $t_{1} z t_{2}$ vor, was für die Güte der an zugrunde liegender Stichprobe erhobener Daten spricht.

\subsubsection{Entwicklungsstabilitäten}

Im längsschnittlichen Untersuchungsdesign wurden im durchschnittlichen Alter von 22 Monaten, 4;0 Jahren, 5;0 Jahren und 7;10 Jahren mit den bewährten klinischen Verfahren Wortschatzleistungen, Satzgedächtnisfähigkeiten sowie die rezeptiven und expressiven grammatischen Leistungen untersucht und die Ergebnisse in den entsprechenden Untertests über alle Zeitspannen für jeden Sprachaspekt korreliert. Hierbei gehen in die Korrelationsanalysen für den Wortschatz $\mathrm{zu}_{\mathrm{0}}$ der Prozentrang für den expressiven 
Wortschatzumfang im ELAN, $\mathrm{zu}_{1}$ und $\mathrm{t}_{2}$ die Rohwerte im AWST3-6 und zu t 3 die Rohwerte aus dem Untertest „Wortschatz“ (HAWIK III) ein. Für die rezeptiven grammatischen Fähigkeiten werden zu $t_{1}$ und $t_{2}$ die Rohwerte des Untertests „Verstehen von Sätzen“ (SETK 3-5) und zu t 3 die Rohwerte im Untertest „Verstehen grammatischer Strukturformen“ (HSET) als Variablen in den Korrelationsanalysen verwendet. Bezogen auf die expressiven grammatischen Leistungen fungieren die Summe aller Pluralwörter im ELAN zu $t_{0}$, die Rohwerte im Untertest „morphologische Regelbildung“ (SETK 3-5) $\mathrm{zu} \mathrm{t}_{1}$ und $\mathrm{t}_{2}$ sowie die Rohwerte aus dem Untertest „Plural-Singular-Bildung“ (HSET) $\mathrm{zu} \mathrm{t}_{3}$ als Variablen in den Korrelationsanalysen. Für das Satzgedächtnis werden die Rohwerte im Untertest „Satzgedächtnis“ (SETK 3-5) $\mathrm{zu} \mathrm{t}_{1}$ und $\mathrm{t}_{2}$ sowie die Rohwerte im Untertest „Imitation grammatischer Strukturformen“ (HSET) miteinander korreliert. Es wurden Pearson ProduktMoment-Korrelationen berechnet und (einseitig) auf Signifikanz geprüft.

Da sich eine Vielzahl von empirischen Vorhersagen zur Prüfung der psychologischen Hypothesen für alle Sprachaspekte über alle Zeitspannen ableiten lässt, werden im Folgenden exemplarisch die empirischen Vorhersagen für den Sprachaspekt Wortschatz benannt. Alle weiteren empirischen Vorhersagen sind entsprechend aufgebaut und werden deshalb nicht explizit formuliert.

Empirische Vorhersagen:

EV 1: Es wird eine signifikante positive Korrelation für den Prozentrang im ELAN zu $\mathrm{t}_{0}$ und die Rohsumme im AWST 3-6 zu t $\mathrm{t}_{1}$ erwartet.

EV 2: Es wird eine signifikante positive Korrelation für den Prozentrang im ELAN zu t $\mathrm{t}_{0}$ und die Rohsumme im AWST 3-6 zu t $\mathrm{t}_{2}$ erwartet.

EV 3: Es wird eine signifikante positive Korrelation für den Prozentrang im ELAN zu to und die Rohsumme im Untertest Wortschatz (HAWIK III) zu t ${ }_{3}$ erwartet.

EV 4:Es wird eine signifikante positive Korrelation für die Rohwerte im AWST 3-6 zu t und $t_{2}$ erwartet.

EV 5: Es wird eine signifikante positive Korrelation für die Rohwerte im AWST 3-6 zu t und die Rohsumme im Untertest Wortschatz (HAWIK III) zu t 3 erwartet.

EV 6: Es wird eine signifikante positive Korrelation für die Rohwerte im AWST 3-6 zu t 2 und die Rohsumme im Untertest Wortschatz (HAWIK III) zu t 3 erwartet. 
Abbildung 6 veranschaulicht die Ergebnisse der Korrelationsanalysen. Hierbei ist die graphische Darstellung (mit Pfeilen) nicht kausal zu interpretieren, sondern dient allein der Veranschaulichung der zeitlichen Dimension.

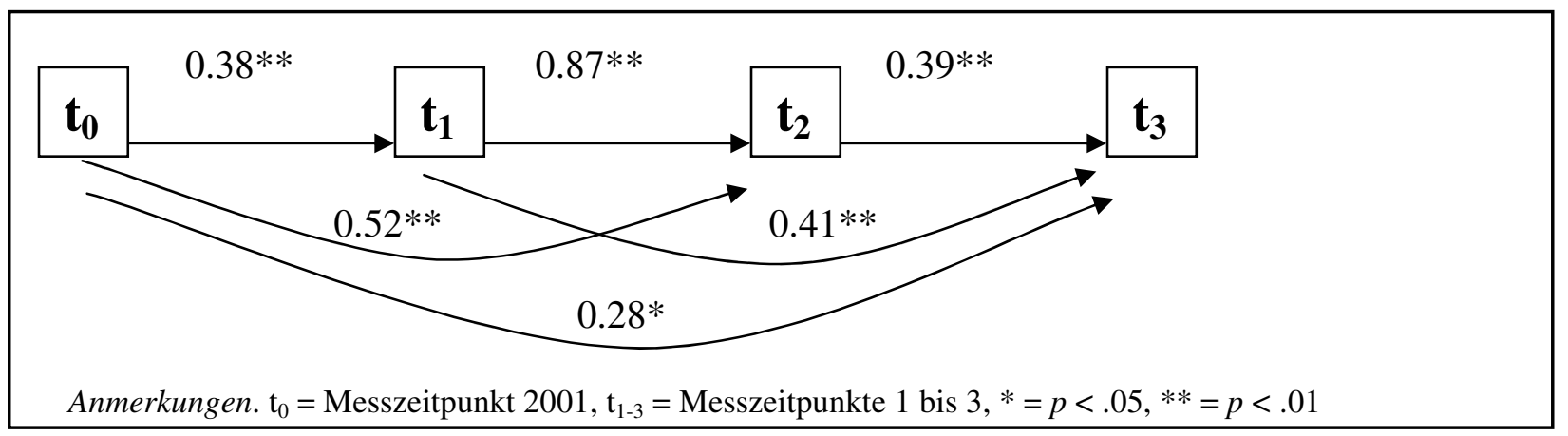

Abbildung 6

Stabilitäten für den Wortschatz, von $t_{0}$ bis $t_{3}$

Die Ergebnisse zeigen, dass über alle Messzeitpunkte signifikante Korrelationen in der Höhe von .28 bis .87 vorlagen. Die niedrigste Korrelation ergab sich über die größte Zeitspanne (von $\mathrm{t}_{0} \mathrm{zu}_{3}$ ) und die höchste Korrelation zeigte sich beim geringsten zeitlichen Untersuchungsabstand $\left(t_{1}\right.$ und $\left.t_{2}\right)$ und der Korrelation von Rohsummen aus dem gleichen Untersuchungsverfahren (AWST 3-6).

Für das Konstrukt „rezeptive grammatische Leistungen“ wurden zu $\mathrm{t}_{1}$ und $\mathrm{t}_{2}$ die Rohwerte des Untertests „Verstehen von Sätzen“ aus dem SETK 3-5 verwendet. Zu t t $_{1}$ sah der SETK 3-5 unterschiedliche Items (Anzahl und Art) für den Untertest VS für dreijährige und vierjährige Kinder vor (siehe Kapitel 3.2). Die Ergebnisse im Untertest VS (Rohwerte) waren zu $t_{1}$ nicht für die gesamte Stichprobe vergleichbar, so dass die Korrelationen mit späteren Maßen des Sprachverstehens getrennt für Drei- und Vierjährige berechnet werden mussten.

Die errechneten Pearson Produkt-Moment-Korrelationen sind in Abbildung 7 dargestellt. 


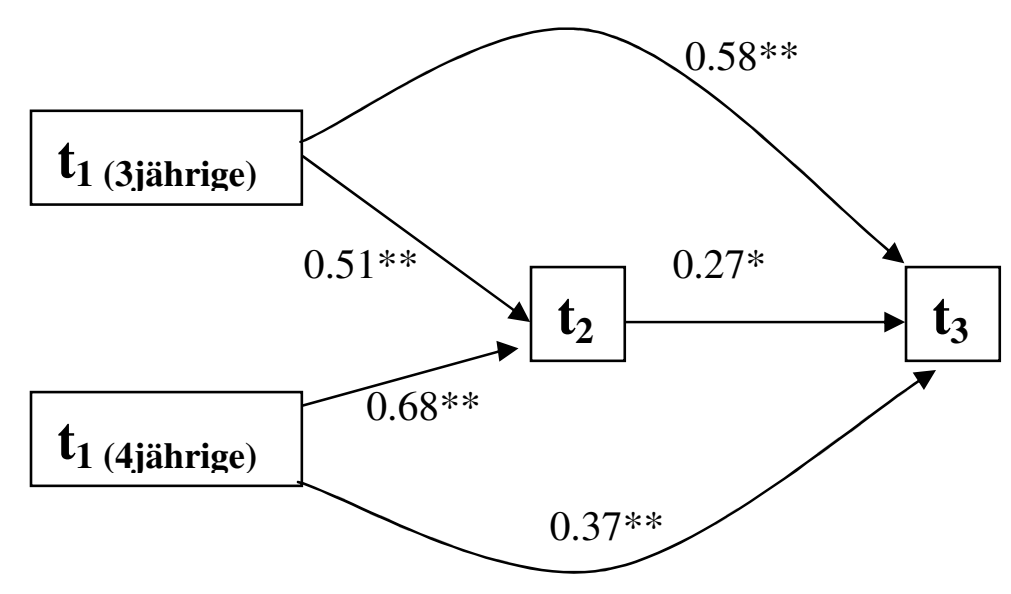

Anmerkungen. $\mathrm{t}_{1-3}=$ Messzeitpunkte 1 bis $3, *=p<.05, * *=p<.01$

Abbildung 7

Stabilitäten für die rezeptiven grammatischen Leistungen von $t_{1}$ bis $t_{3}$

Signifikante Korrelationen über alle Untersuchungszeitpunkte $(\operatorname{Min}=.27$, Max = .68) liegen vor. Die höchste Korrelation bestand für die geringste Zeitspanne und die Verwendung des gleichen Messinstruments von $\mathrm{t}_{1} \mathrm{zu}_{2} \mathrm{t}_{2}$ allerdings nur für die Stichprobe der vierjährigen Kinder. Die Werte der dreijährigen Kinder $\mathrm{zu} \mathrm{t}_{1}$ korrelierten jedoch deutlich höher mit den Werten $\mathrm{zu} \mathrm{t}_{3}(r=.58)$ als die der vierjährigen $(r=.37)$ Kinder $\mathrm{zu} \mathrm{t}_{1}$. Die geringste Korrelation konnte für die Werte von $\mathrm{t}_{2} \mathrm{zu} \mathrm{t}_{3}$ berechnet werden $(r=.27)$.

Für das Konstrukt „expressive grammatische Leistungen“ wurden $\mathrm{zu} \mathrm{t}_{1}$ und $\mathrm{t}_{2}$ die Rohwerte des Untertests „Morphologische Regelbildung“ als Variablen verwendet. Zu t $\mathrm{t}_{1}$ sah der SETK 3-5 unterschiedliche Items (Anzahl und Art) für den Untertest MR für dreijährige und vierjährige Kinder vor (siehe Kapitel 3.2), so dass die Korrelationen mit früheren und späteren Maßen der morphologischen Regelbildung für die drei- und vierjährigen Kinder separat berechnet werden mussten. 
Die entsprechenden Pearson Produkt Moment Korrelationen zeigt Abbildung 8.

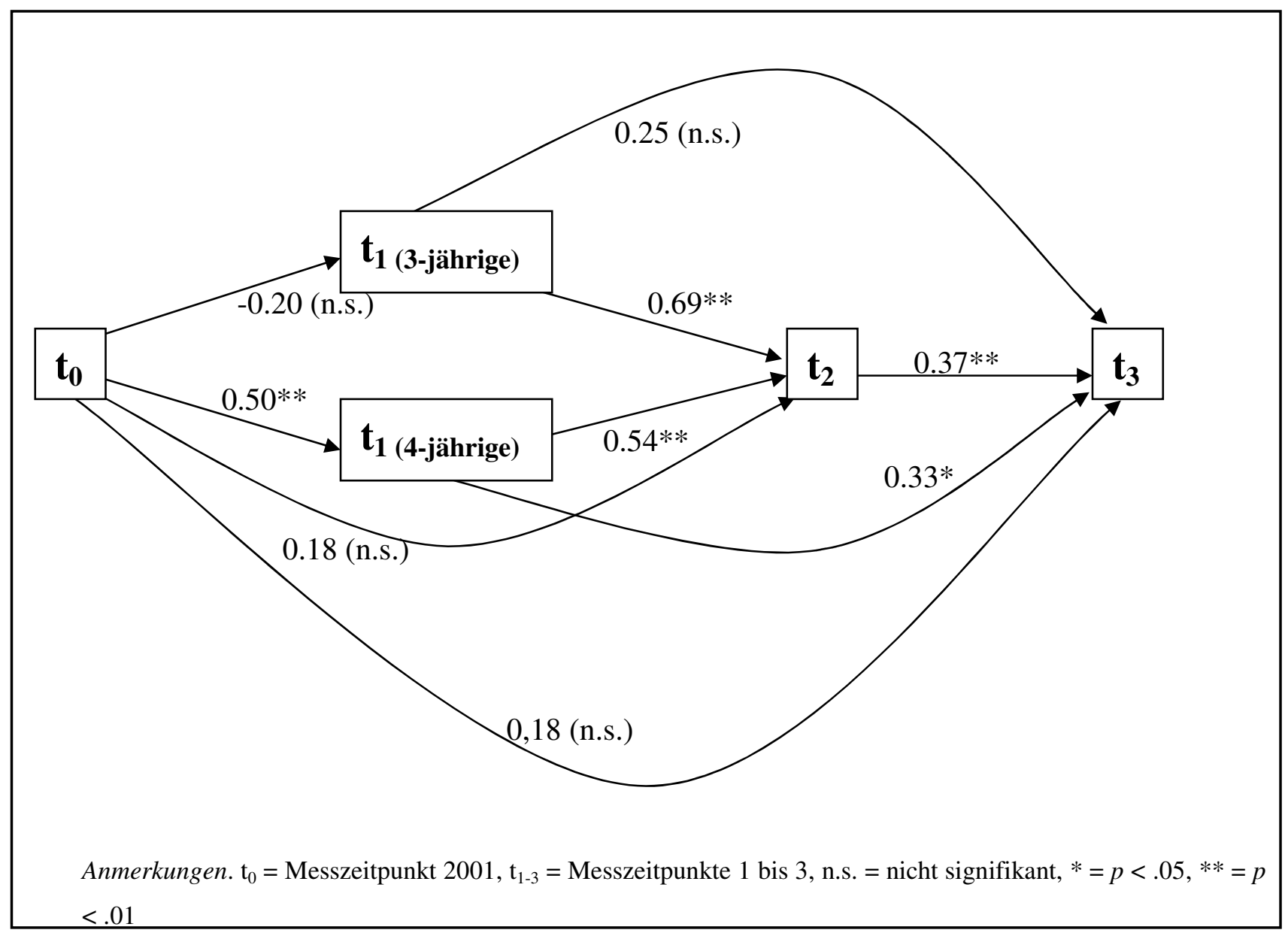

Abbildung 8

Stabilitäten für die expressiven grammatischen Leistungen von $t_{0}$ bis $t_{3}$

Die signifikanten Korrelationen erreichen Werte von .33 bis .69. Es wird deutlich, dass nicht für alle Untersuchungsintervalle signifikante Korrelationen vorliegen. So korreliert die „Pluralsumme“ zu to einzig signifikant mit den Werten der vierjährigen Kinder zu $\mathrm{t}_{1}(r=.50)$. Die Werte der Dreijährigen zu t $\mathrm{t}_{1}$ korrelieren lediglich signifikant mit den Werten $\mathrm{zu}_{2} \mathrm{t}_{2}(r=$ .69) und dieses tendenziell höher als die Werte der Vierjährigen $(r=.53)$. Die Werte der Vierjährigen zu $\mathrm{t}_{1}$ korrelieren signifikant mit den Werten aller anderen Messzeitpunkte. Auch hier gilt, dass die höchsten Korrelationen zwischen $t_{1}$ und $t_{2}$ berechnet wurden. 
Abbildung 9 stellt die sich ergebenden Pearson Produkt Moment Korrelationen für das Satzgedächtnis dar.

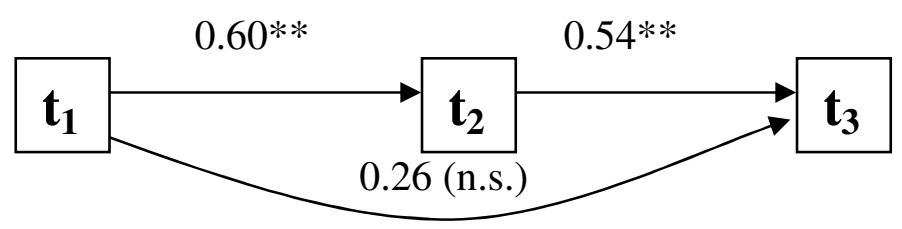

Anmerkungen. $\mathrm{t}_{1-3}=$ Messzeitpunkte 1 bis 3 , n.s. $=$ nicht signifikant, $*=p<.05, * *=p<.01$

Abbildung 9

Stabilitäten für das Satzgedächtnis von $t_{1}$ bis $t_{3}$

Für das „Satzgedächtnis“ sind die Korrelationen von $\mathrm{t}_{1} \mathrm{zu} \mathrm{t}_{3}$ nicht signifikant. $\mathrm{Zu}$ den anderen Zeitspannen $\mathrm{t}_{1} \mathrm{zu} \mathrm{t}_{2}(r=.56)$ und $\mathrm{t}_{2} \mathrm{zu} \mathrm{t}_{3}(r=.54)$ sind die signifikanten Korrelationen vergleichbar hoch.

\subsection{Diskussion}

Die psychologische Hypothese 1 (Für ein Kind mit relativ guten Wortschatzleistungen [Rangposition innerhalb einer Bezugsgruppe] in der frühen Kindheit werden auch relativ gute Wortschatzleistungen im Alter von 4;0, 5;0 und 7;10 Jahren erwartet. Die gleiche Erwartung gilt für die Zeitspanne von 4;0 zu 5;0 Jahren und von 4;0 zu 7;10 Jahren sowie von 5;0 zu 7;10 Jahren.) konnte bei strenger Prüfung (konjunktive Verknüpfung aller empirischen Vorhersagen) bestätigt werden. Wortschatz kann somit als ein stabiles sprachliches Merkmal über die Zeitspanne von früher Kindheit bis Grundschulzeit bezeichnet werden.

Die psychologische Hypothese 2 (Für ein Kind mit relativ guten rezeptiven grammatischen Leistungen [Rangposition innerhalb einer Bezugsgruppe] im Alter von 4;0 Jahren werden ebenfalls relativ gute rezeptive grammatische Leistungen im Alter von 5;0 und 7;10 Jahren erwartet. Die gleiche Erwartung gilt für die Zeitspanne von 5;0 Jahren zu 7;10 Jahren.) bestätigte sich bei strenger Prüfung trotz Stichprobenteilung $\mathrm{zu} \mathrm{t}_{1}$. Rezeptive grammatische Leistungen lassen sich auf vorliegender Datenbasis als stabile sprachliche Leistungen von Vorschul- bis Grundschulalter charakterisieren.

Die psychologische Hypothese 3 (Für ein Kind mit relativ guten expressiven grammatischen Fähigkeiten (Rangposition innerhalb einer Bezugsgruppe) in der frühen 
Kindheit werden auch relativ gute expressive grammatische Fähigkeiten im Alter von 4;0, 5;0 und 7;10 Jahren erwartet. Die gleiche Erwartung gilt für die Zeitspanne von 4;0 zu 5;0 Jahren und von 4;0 zu 7;10 Jahren sowie von 5;0 zu 7;10 Jahren.) konnte bei strenger Prüfung mit konjunktiver Verknüpfung der empirischen Vorhersagen nicht bestätigt werden, da für die expressiven grammatischen Fähigkeiten nicht hinsichtlich aller Teilstichproben und über alle Zeitintervalle signifikante Korrelationen in den Ergebnissen bestanden. Die expressiven grammatischen Fähigkeiten wurden wie der Wortschatz von $t_{0}$ bis $t_{3}$ über eine große Altersspanne von bis zu 6 Jahren erhoben. Die Ergebnisse $\mathrm{zu} \mathrm{t}_{0}$ korrelierten lediglich signifikant mit den Ergebnissen der vierjährigen Kinder $\mathrm{zu} \mathrm{t}_{1}$. Dieses wird als Hinweis auf eine Schwäche in der Operationalisierung des Konstrukts $\mathrm{zu} \mathrm{t}_{0}$ gewertet. Morphologische Regelbildung war $\mathrm{zu} \mathrm{t}_{0}$ nicht mittels eines bewährten klinischen Verfahrens in Bezug auf dieses Untersuchungsmerkmal erhoben worden. Stellvertretend war die Summe der Pluralwörter (ELAN), die ein Kind im Alter von 16 bis 26 Monaten spricht, als Maß für die morphologische Regelbildung verwendet worden. Hintergrund war die Logik, dass ein Kind, welches Pluralwörter im expressiven Wortschatz zur Verfügung hat, auch in der Lage sein muss, diese zu bilden. Für die Menge der Pluralwörter im ELAN gilt jedoch zum einen, dass die geringe Anzahl von 7 Items wenig reliable Messungen erwarten lässt und zum anderen Pluralwörter wie „Zähne“ und „Nudeln“ solche sind, die mehrheitlich in der Pluralform in der Alltagssprache vorkommen und nur in geringem Maße Fähigkeiten zur morphologischen Markierung bestehender Wörter im Lexikon repräsentieren. Für eine Operationalisierung früher expressiver grammatischer Leistungen durch die Pluralsumme im ELAN sprach jedoch die substanzielle Korrelation von .63 ( $p=.00)$ mit der MLU zu to sowie das Bestreben, gut vergleichbare Operationalisierungen des Konstruktes „Expressive grammatische Fähigkeiten“ von $t_{0}$ bis $t_{3} \mathrm{zu}$ finden. Von einem Einbezug der errechneten Korrelationen aus der Pluralsumme im ELAN zu t $\mathrm{t}_{0}$ für die Rückschlüsse auf die psychologische Hypothese sollte aus den berichteten Gründen abgesehen werden und nach valideren und reliableren Methoden zur Erfassung der morphologischen Regelbildung im frühen Kindesalter gesucht werden.

Bedenken bestehen auch für die Bewertung der Ergebnisse $\mathrm{zu}_{1}$ für die dreijährigen Kinder. Dieses ist die einzige Stichprobe, bei der über die Zeitspanne von $t_{1}$ bis $t_{3}$ keine morphologische Regelbildung an Kunstwörtern erhoben wird, sondern ausschließlich an Alltagswörtern, was die Vergleichbarkeit der Ergebnisse deutlich einschränkt und das nicht signifikante Ergebnis erklären mag. 
Schlussfolgernd wird für die morphologische Regelbildung als einer Operationalisierung der expressiven grammatischen Leistungen für die Zeitspanne von 4;0 Jahren bis 7;10 Jahren vom Vorliegen substanzieller normativer Stabilitäten ausgegangen.

Die psychologische Hypothese 4 (Für ein Kind mit relativ guten Satzgedächtnisleistungen [Rangposition innerhalb einer Bezugsgruppe] im Alter von 4;0 Jahren werden auch relativ gute Satzgedächtnisfähigkeiten im Alter von 5;0 und 7;10 Jahren erwartet. Die gleiche Erwartung gilt für die Zeitspanne von 5;0 zu 7;10 Jahren.) konnte an vorliegendem Datensatz nicht bestätigt werden, denn für die Zeitspanne von $t_{1} z t_{3}$ lagen keine signifikanten Korrelationen vor. Die Zeitspanne von über drei Jahren ist im Rahmen der kindlichen Entwicklung immens und so wundert es einerseits nicht, dass für diesen Zeitraum keine signifikanten korrelativen Zusammenhänge vorliegen. Andererseits bestehen sowohl von $\mathrm{t}_{1} \mathrm{zu}$ $t_{2}$ als auch von $t_{2}$ zu $_{3}$ mit .60 und .54 signifikante Korrelationen hoher Effektstärke, so dass folglich für das Untersuchungsintervall $t_{1}$ bis $t_{3}$ signifikante Korrelationen $\mathrm{zu}$ erwarten gewesen wären. Ein Wechsel der Erhebungsinstrumente liefert keinen Erklärungsgewinn, denn auch von $t_{2} z t_{3}$ wurde vom SETK 3-5 zum HSET gewechselt und dennoch liegen signifikante Korrelationen vor. Die Stichprobe $\mathrm{zu}_{\mathrm{u}}$ bestand allerdings nur aus den vierjährigen Kindern, da für die dreijährigen Kinder der Untertest Satzgedächtnis nicht vorgesehen war und somit wurden die Korrelationen von $t_{1} \mathrm{zu} \mathrm{t}_{3}$ nur für eine kleine Stichprobe ermittelt. Dieses erklärt am ehesten, warum für die Zeitspanne von $t_{1} z t_{3}$ keine signifikanten Korrelationen errechnet werden konnten und schränkt den direkten Rückschluss von den Ergebnissen auf die psychologische Hypothese ein. Für eine ungeteilte Stichprobe zu $\mathrm{t}_{1}$ wären auch für das Satzgedächtnis durchgängig signifikante Korrelationen von 4;0 bis 7;10 Jahren zu erwarten gewesen.

Ingesamt zeigten sich die unterschiedlichen Sprachaspekte über eine Vielzahl von Untersuchungsintervallen als Merkmale von substanzieller differentieller Stabilität. Dieses ist um so bemerkenswerter, wenn man die großen Zeitspannen von bis zu 6 Jahren in Betracht zieht und bedenkt, dass es bei der Erhebung der Daten über die Zeit mehrfach zu einem Wechsel der Erhebungsinstrumente kam, der aufgrund der großen Altersspanne nicht vermeidbar war. Für Wortschatz und expressive grammatische Fähigkeiten ergaben sich zwei Wechsel bzgl. der Untersuchungsverfahren und für Satzgedächtnis und rezeptive grammatische Fähigkeiten jeweils einer. 
Neben der Frage, ob Sprache überhaupt ein Merkmal von bedeutsamer differentieller Stabilität von früher Kindheit bis ins Grundschulalter ist, interessiert auch die Frage nach der Ausprägung dieser Stabilitäten. Wie stabil ist z.B. der Wortschatz von 4;0 bis 5;0 Jahren? Zur Beantwortung dieses Fragenkomplexes werden die Ergebnisse mit Hinblick auf die Konventionen von Cohen (1988) bewertet.

Die höchste mittlere Korrelation $(r=.65)$ ergab sich erwartungskonform für das kleinste Zeitfenster von 4;0 bis 5;0 Jahren. Für diesen Zeitraum bewährte sich Sprache hinsichtlich aller Aspekte als hochstabiles Merkmal ( $r=.51-.87)$, wobei der Wortschatz mit 87 als besonders stabiler Sprachaspekt hervortrat. Die Werte für die gleiche Zeitspanne betrugen in der Untersuchung von Weber et al. (2005) an einer größeren Stichprobe von $\mathrm{n}=111$ deutschsprachigen Kindern bei Verwendung gleicher Testverfahren (mit Ausnahme der Wortschatzerfassung) .51 für den Wortschatz und .73 für den Bereich Grammatik (Untertest VS, MR und SG). Die unterschiedlichen Korrelationen für den Bereich Wortschatz $(r=.51$ vs. $r=.87$ ) können durch die Verwendung unterschiedlicher Testverfahren erklärt sein. In der Studie von Weber et al. (2005) wurden die Ergebnisse in den Untertests des SETK 3-5 zu einem Grammatikwert verrechnet. Dieses kann neben der kleineren Stichprobe in vorliegender Untersuchung (insbesondere durch die Teilung der Stichproben in drei- vs. vierjährige Kinder $\mathrm{zu} \mathrm{t}_{1}$ ) $\mathrm{zu}$ den höheren Korrelationen für den Bereich Grammatik in der Studie von Weber et al. (2005) geführt haben. Als Fazit gilt jedoch, dass sich Sprache für die Zeitspanne von 4;0 bis 5;0 Jahren als hochstabiles Merkmal in beiden Studien präsentiert hat und dass die auf der vorliegenden Datenbasis ermittelten Ergebnisse gut vergleichbar mit anderen Forschungsergebnissen sind. Folglich erlauben die mit 4 Jahren erhobenen Sprachmaße gute Vorhersagen für die Sprachentwicklung (bzgl. der gleichen sprachlichen Aspekte) mit 5 Jahren.

Die mittlere Korrelation für das Untersuchungsintervall von 5;0 bis 7;10 Jahren beträgt .39 und fällt im Vergleich zum Zeitfenster 4;0 bis 5;0 Jahre geringer aus. Dieses lässt sich zunächst erklären aus der größeren Zeitspanne von 2;10 Jahren, für die Aussagen gemacht werden. Erschwerend kommt hinzu, dass für das Untersuchungsintervall von $t_{2} z t_{3}$ ein Wechsel der Untersuchungsverfahren für alle Sprachaspekte vollzogen wurde. Auffallend hoch korrelierten die Werte im Untertest Satzgedächtnis zu t $\mathrm{t}_{2}$ mit denen zu $\mathrm{t}_{3}(r=.54)$. Dieses könnte als Hinweis dahingehend interpretiert werden, dass Satzgedächtnis in besonderem Maße von kognitiven Faktoren geprägt ist, die für das Alter von 5;0 bis 7;11 Jahren eine höhere differentielle Stabilität aufweisen als rein sprachliche Faktoren. 
Insgesamt lässt sich aus den Ergebnissen ableiten, dass Sprache im Alter von 5;0 bis 7;10 von moderater differentieller Stabilität ist. Vorhersagen späteren Sprachoutcomes für dieses Zeitfenster sind von geringerer Güte als Vorhersagen für das Zeitintervall 4;0 bis 5;0 Jahren.

Das Untersuchungsintervall von $4 ; 0$ bis $7 ; 10$ Jahren weist nicht für alle Sprachkomponenten signifikante Korrelationen der Ergebnisse auf (vgl. Satzgedächtnis und expressive grammatische Fähigkeiten dreijähriger Kinder $\left.\mathrm{zu} \mathrm{t}_{1}\right)$. Die signifikanten Korrelationen erreichen Werte von .33 - .58. Dieses ist gut vergleichbar mit der Studie von Bornstein et al. (2006), in der für die Zeitspanne von 4;6 bis 6;10 Jahren $(n=84)$ Korrelationen in Höhe von .25 -.55 berichtet werden. Allerdings beziehen sich diese auf ein geringeres Zeitintervall. Weber et al. (2005) fanden in ihrer Untersuchung für ein besser vergleichbares Zeitfenster von 4;0 bis 7;9 Jahren Korrelationen für den Wortschatz von .35, was gut übereinstimmt mit den in dieser Dissertationsstudie errechneten Korrelationen von .41. Für die grammatischen Leistungen benennen Weber et al. (2005) höhere Korrelationen ( $r$ $=.56)$ als in hiesiger Studie $(r=.43)$.

Im Mittel konnten für die untersuchten Sprachaspekte (mit Ausnahme des Satzgedächtnisses) bezogen auf das Untersuchungsintervall von $4 ; 0$ bis $7 ; 10$ Jahren Stabilitäten mittlerer Effektstärke errechnet werden. Dieses Ergebnis ist erstaunlich, wenn man bedenkt, dass zum einen kleine Stichproben $\mathrm{zu} \mathrm{t}_{1}$ vorlagen, die Untersuchungsverfahren wechselten und zudem ein Zeitfenster von fast 4 Jahren betrachtet wurde. Studien an größeren Stichproben unterstützen dieses Ergebnis und berichten von mittleren bis hohen Stabilitäten.

In den bisherigen Ausführungen wurde auf das Zeitfenster von $t_{0}$ ausgehend nicht Bezug genommen, da fundierte Aussagen zu diesem Zeitfenster nur bezogen auf Wortschatzdaten möglich sind. Für den Wortschatz liegt für die Zeitspanne von 22 Monaten bis 7;10 Jahren (94 Monaten) eine moderate Stabilität vor $(r=.28)$. Dieses ist in Anbetracht des großen Zeitintervalls von 72 Monaten ein hoher Wert, der insbesondere Wortschatz als eine stabile sprachliche Leistung über eine große Zeitspanne kennzeichnet.

Welche Aussagen lassen sich aus den Ergebnissen zur Frage ableiten, wie stabil die unterschiedlichen sprachlichen Aspekte sind?

Für die rezeptiven grammatischen Fähigkeiten ergeben sich Stabilitäten mittlerer bis hoher Effektstärke. Schwer zu erklären sind die höheren Stabilitäten für das Zeitfenster von 4;0 bis $7 ; 10$ Jahren ( $r=.37$ und .58) im Vergleich zum Untersuchungsintervall von $5 ; 0$ bis 7;10 
Jahren $(r=.27)$, denn es wurden identische Testverfahren verwendet und die Stichprobengrößen zu t $t_{1}$ waren kleiner als $\mathrm{zu}_{2}$.

Die expressiven grammatischen Fähigkeiten zeichnen sich ebenso durch Stabilitäten mittlerer bis hoher Effektstärke von 4;0 bis 7;10 Jahren aus und das Satzgedächtnis verfügt über hohe Stabilitäten von 4;0 bis $5 ; 0$ Jahren und von $5 ; 0$ bis $7 ; 10$ Jahren. Da auch der Wortschatz sogar über die Zeitspanne von 22 Monaten bis 7;10 Jahren über differentielle Stabilitäten mittlerer bis hoher Effektstärke verfügt, kann sich der Aussage von Bornstein et al. (2004) angeschlossen werden, der für das Alter von 1 bis 7 Jahren von moderaten bis hohen Stabilitäten für die Sprache ausgeht. Die vorliegende Untersuchung ist eine der wenigen Längsschnittstudien im deutschen Sprachraum, die Aussagen zur Stabilität von Sprache über die Altersspanne von 1 bis 8 Jahren möglich macht.

Die Stabilität von Entwicklungsmerkmalen über eine längere Zeitspanne ist ein Forschungsgegenstand, der höchste Ansprüche an die methodische Umsetzung der Forschungsfragen stellt. Nur bei sorgfältiger und gut vergleichbarer Operationalisierung der untersuchten Merkmale sowie der Auswahl gut vergleichbarer Messinstrumente für das gesamte Zeitfenster repräsentieren die Ergebnisse wirklich z.B. die Stabilität von Sprache und nicht die Stabilität der Erfassung von Sprache. Deshalb lag das besondere Bestreben in dieser Studie darin, gut vergleichbare Operationalisierungen der Sprachaspekte über die gesamte Untersuchungsspanne von 72 Monaten zu realisieren und besondere Sorgfalt bei der Datenerhebung und Auswertung hinsichtlich ihrer Vergleichbarkeit über die Zeit walten zu lassen.

Die Abhängigkeit der Güte von Erkenntnissen von der Güte ihrer Erhebung, und hier spielen noch eine ganze Reihe weiterer Faktoren (wie z.B. die Stichprobengröße) eine entscheidende Rolle, wird bei der Untersuchung von Stabilitäten von Merkmalen überdeutlich, gilt jedoch für alle Bereiche der psychologischen Forschung und verlangt nach einer Intensivierung der Bemühungen bei der Entwicklung adäquater Erhebungsverfahren für eine große Altersspanne. 


\section{Der frühe Wortschatz als Prädiktor für späteres Sprachoutcome}

\subsection{Theoretischer Hintergrund}

Die frühe Vorhersage von Sprachoutcome ist von hoher praktischer und theoretischer Relevanz. Verzögerungen und Störungen der Sprachentwicklung gelten als eines der Hauptgesundheitsprobleme im Kindesalter (Paul \& Kellogg, 1997), wobei von einer großen Anzahl unentdeckter Fälle (bis zu einem Drittel) auszugehen ist (Tomblin et al., 1997). Deutliche Zusammenhänge von frühen Sprachentwicklungsabweichungen (bis 3 Jahren) mit persistierenden Sprachstörungen, Lern- und Schulproblemen sowie sozialen und Verhaltensproblemen in späterem Alter sind nachgewiesen (Ward, 1999). Die frühe Vorhersage späterer Sprachleistungen könnte somit entscheidend dazu beitragen, die weitere Sprachentwicklung eines Kindes schon in frühem Alter $\mathrm{zu}$ beurteilen, ggf. frühzeitig geeignete Interventionen einzuleiten und somit das Risiko für die Entwicklung von Sprachstörungen und anderen Entwicklungsstörungen zu minimieren.

Die Vorhersage späterer Sprachleistungen für den regelrechten Spracherwerb ist vor allem von großer theoretischer Bedeutung. Lässt sich schon aus den frühen Sprachmaßen z.B. im Alter von 2 Jahren gut die weitere sprachliche Entwicklung z.B. im Alter von 5 oder 8 Jahren vorhersagen, dann ermöglicht dieser Befund vor allem Einblicke in die Mechanismen, welche generell dem Spracherwerb zugrunde liegen und kann theoriebildend bzw. -formend sein. Denn gute Vorhersagen späterer Leistungen deuten auf eine hohe Stabilität eines Merkmals hin. So wäre Sprache als besonders stabiles Merkmal über eine lange Zeitspanne zu charakterisieren, wenn die Vorhersagen aus Sprachmaßen mit 2 Jahren gut mit den tatsächlichen sprachlichen Leistungen im Alter von 8 Jahren übereinstimmen. Dieses Befundmuster gilt z.B. für die kognitive Entwicklung nicht (Spiel et al., in Druck) und ließe Rückschlüsse im Vergleich zu. Bei der kognitiven Entwicklung eines Kindes wäre von dem Bestehen anderer Wirkmechanismen auszugehen als bei der Sprachentwicklung.

Die genetische Bedingtheit des Spracherwerbs wird in den letzen Jahren immer wieder hervorgehoben (z.B. Dionne, Dale, Boivin \& Plomin, 2003). Bei einer alleinigen genetischen Bedingtheit müsste sich Sprache jedoch als hoch stabiles Merkmal erweisen, welches schon früh sehr gute Vorhersagen für späteres Sprachoutcome ermöglicht. Eingeschränkte prädiktive Validität früher Sprachmaße würde die Annahme unterstützen, dass Spracherwerb ein hochkomplexes multifaktoriell bedingtes Entwicklungsphänomen ist.

Die möglichen theoretischen Rückschlüsse aus den Untersuchungen zur frühen Vorhersage von Sprachoutcome für den gesunden Spracherwerb können sich wiederum auf die Interventionsmaßnahmen bei Sprachstörungen auswirken, denn die zugrunde liegenden 
Mechanismen der gesunden Sprachentwicklung sind hilfreich, um Zeitpunkt, Frequenz und auch inhaltlicher Struktur von effektiven Interventionsmaßnahmen zu planen. Praktische Relevanz haben Vorhersagen bezogen auf den regelrechten Spracherwerb zum Beispiel mit Hinblick auf die Schuleingangsuntersuchungen. Sollten Untersuchungen zur prädiktiven Validität unterschiedlicher Sprachmaße im Vorschulalter ergeben, dass es geeignete Zeitfenster und Sprachmaße gibt, die zu guten Vorhersagen späterer grundlegender Sprachleistungen für eine erfolgreiche Schullaufbahnbewältigung führen, könnte entscheidend zur Verbesserung der Chancen vieler Kinder für einen erfolgreichen Start in der Schule beigetragen werden.

Sowohl für die praktischen als auch für die theoretischen Implikationen, die sich aus Untersuchungen zur prädiktiven Validität früher Sprachmaße ableiten lassen, stellen die ersten drei Lebensjahre des Kindes das entscheidende Zeitfenster dar, denn mit drei Jahren ist die Wortschatzkomposition eines Kindes mit der von Erwachsenen vergleichbar (Kauschke, 2003) und der Erwerb grundlegender morpho-syntaktischer Strukturen der Muttersprache ist für die meisten Kinder abgeschlossen.

Wortschatz ist eine Komponente von Sprache, die z.B. in besonders engem Zusammenhang zur grammatischen Entwicklung eines Kindes steht (Conboy \& Thal, 2006). Grimm (2000) bezeichnet Wörter als die zentralen Bausteine der Sprache. Der Wortschatz stellt quasi das Rohmaterial dar, aus dem ein Kind Sätze baut und an dem es seine Wortformenentwicklung vollzieht. Dieses betont insbesondere die Bedeutung des frühen Wortschatzes für die Sprachentwicklung, denn nur das Verfügen über eine gewisse Anzahl an „Bausteinen“ ermöglicht weitere sprachliche Entwicklung wie das Bauen von Sätzen und Verändern von Wortformen.

Der frühe expressive Wortschatz (die Wörter, die ein Kind spricht) hat sich als bester Prädiktor für spätere Sprachentwicklungs- und andere Entwicklungsstörungen etabliert (Fischel et al., 1989) und ist konkret und gut zu beobachten. Fenson et al. (1993) berichten von einer guten prädiktiven Validität des frühen Wortschatzes (erhoben mit Parent Report) für spätere Wortschatz- und Grammatikleistungen (erhoben in standardisierter Testsituation) und Tamis-Lemonda und Bornstein (1994) ergänzen eine gute prädiktive Validität für spätere MLU und semantische Vielfältigkeit des Wortschatzes. Die Wortschatzgröße sagt zudem den Schulerfolg voraus (Toppelberg \& Shapiro, 2000), und das Konstrukt Wortschatzkompetenz ermöglicht eine besonders gute Vorhersage für die spätere kognitive Entwicklung. 
Der rezeptive Wortschatz (die Wörter, die ein Kind versteht) gilt als sehr guter Prädiktor für den expressiven Wortschatz, die spätere allgemeine Sprachentwicklung und die kognitive Entwicklung (Bornstein \& Haynes, 1998). So fällt bei Kindern mit einem eingeschränkten expressiven Wortschatz mit 24 Monaten schon frühzeitig (im Alter von 12 und 18 Monaten) ein geringerer rezeptiver Wortschatz auf (Grimm, 2000). Nach Bornstein und Haynes (1998) korreliert der rezeptive Wortschatz mit 20 Monaten hoch mit dem IQ (verbal und nonverbal) mit 48 Monaten. Andererseits geben Tomasello und Mervis (1994) zu bedenken, dass für Eltern der Verstehensbegriff nicht eindeutig ist und diese somit das Sprachverständnis schwer beurteilen können (Rescorla, 1989), was die Vorhersagekraft des rezeptiven Wortschatzes einschränkt. Weiterhin weisen verschiedene Autoren darauf hin, dass der rezeptive Wortschatz ab einem Alter von 1;6 bis 1;8 Jahren mit unterschiedlichen Methoden nicht mehr reliabel erfasst werden kann (Fenson et al., 1993; Stern \& Stern, 1922). Aus diesen Gründen wird in der Mehrheit aller Studien zur Vorhersage späterer Sprachleistungen aus dem frühen Wortschatz der expressive Wortschatz als Prädiktorvariable erhoben.

Bei der Betrachtung des expressiven Wortschatzes können unterschiedliche Operationalisierungen des Konzeptes Wortschatz zu mehreren möglichen Variablen führen (z.B. Wortschatzumfang: Anzahl aller unterschiedlichen Wörter oder Anzahl aller gesprochenen Wörter mit unterschiedlichem Wortstamm, Anzahl an Verben, Anzahl aller gesprochenen Wörter in einer Aufnahmesequenz; Wortschatzzuwachs). Zumeist wird der expressive Wortschatzumfang als Anzahl aller unterschiedlichen Wörter, die ein Kind spricht, in der aktuellen Forschung verwendet.

Als Erhebungsmethoden kommen standardisiertes Testen, Sprachproben und Elternbericht bzw. „Parent Report“ (Tagebuch und Checklist) zum Einsatz, wobei sich diese Methoden alle durch hohe Übereinstimmung in den Ergebnissen auszeichnen (Bornstein \& Haynes, 1998). Zumeist findet im frühen Alter Parent Report in Form von Checklisten Anwendung, da auf diese Weise zeit- und kostensparend unabhängig von der Untersuchung am Kind ein valides und reliables Bild vom Sprachentwicklungsstand des Kindes entsteht. Zur Güte der Parent Report-Methode allgemein lässt sich sagen, dass Eltern durchaus in der Lage sind sowohl die Norm als auch Normabweichungen in Bezug auf die Sprachentwicklung ihrer Kinder zu erkennen (Goorhuis-Brouwer, 1990). Insbesondere Mütter geben ein zeitkonsistentes, komplexes und organisiertes Bild der Sprachentwicklung ihres Kindes (Bates, 1994). Dale, Bates, Reznick und Morisset (1989) bezeichnen den Parent Report als eine gute und einfache Methode für Klinik und Forschung in der Sprachentwicklung, dessen Nützlichkeit und 
Schichtunabhängigkeit sie für erwiesen erachten. Bornstein und Haynes (1998) und Rescorla, Mirak und Singh (2000) weisen auf die hohe Übereinstimmung der Ergebnisse von Parent Reports mit tatsächlicher Sprachleistung und anderen Sprachindizes hin. Demnach haben die mittels Parent Report gewonnenen Sprachentwicklungsdaten einen hohen prädiktiven Wert für spätere Wortschatz- und Grammatikentwicklung, MLU und semantische Vielfältigkeit sowie spätere Ergebnisse in klinischen Sprachuntersuchungen. Law, Boyle, Harris, Harkness und Nye (2000) schätzen den Parent Report als genauso sensitiv ein wie das klinische Screening. Im Vergleich zur Laboruntersuchung liefert er reliablere und validere Ergebnisse, weil das Kind den Beobachter gut kennt und dieser das Kind in vielen unterschiedlichen Situationen erlebt, mit denen das Kind vertraut ist und in denen es Dingen begegnet, über die es zu sprechen motiviert ist (Dale, 1991).

So erwiesen sich bei Kindern im Alter von 20 Monaten mittels Elternfragebogen erhobene Wortschatzeinschätzungen in höherem Maße als prädiktiv für die MLU mit 26 Monaten als der tatsächlich beobachtete Wortschatz (Snyder, Bates \& Bretherton, 1981). Die Autoren betonen, dass Mütter das beurteilen, was ihr Kind tatsächlich weiß und nicht nur das, was es aktuell gebraucht. Fenson et al. (1993, S. 4) formulieren demgemäß: „Parent report is less influenced than direct clinical observation by performing factors (such as word frequency)“. Auch ist es nach Bates und Carnevale (1993) schwierig, die frühe Sprachentwicklung überhaupt mittels einer anderen Methode zu erfassen, da es sich bei ersten kindersprachlichen Äußerungen um ein neues, seltenes und unvorhergesehenes Verhalten handelt.

Gibt es ein geeignetes Alter, in dem die Erhebung des frühen Wortschatzes zu besonders guten Vorhersagen späteren Sprachoutcomes führt? Diese Frage wird in der Forschungsliteratur divergent beantwortet. Der Erhebungszeitpunkt für den frühen Wortschatz in den einschlägigen Untersuchungen variiert von 7 bis ca. 36 Monaten.

Insgesamt ist über den prognostischen Wert der Sprachentwicklung unter 12 Monaten wenig bekannt. Weindrich, Jennen-Steinmetz, Rellum, Laucht \& Schmidt (2005) untersuchten im Rahmen einer prospektiven Risikokinderstudie (Mannheimer Risikokinderstudie) bei nicht behinderten Kindern $(n=95)$ vom Kleinkindalter (10 Monate) bis zum Vorschulalter (4;6 Jahre) expressive und rezeptive Sprachleistungen und berichten von besonders hohen Korrelationen früher Sprachmaße (auch rezeptive Wortschatzmaße) im Alter von 10 Monaten zu Sprachoutcome im Alter von 24 Monaten $(r=.63)$ und 54 Monaten $(r=.42)$. In ihrer Studie kamen jedoch veraltete Testverfahren zum Einsatz und die Stichprobe bestand ausschließlich aus Risikokindern mit vielfältigen Entwicklungsrisiken. 
Fenson et al. (1993) untersuchten 62 unauffällige Kinder zu den Testzeitpunkten $t_{1}=8-10$ Monate und $t_{2}=14-17$ Monate. Die Korrelation für den Wortschatzumfang $\mathrm{zu} \mathrm{t}_{1}$ und $\mathrm{t}_{2}$ betrug .38. Die abweichenden Ergebnisse von Fenson et al. (1993) und Weindrich et al. (2005) geben zu bedenken, dass Untersuchungen an Stichproben normal entwickelter Kinder und Untersuchungen an klinischen Stichproben nur bedingt vergleichbar sind.

Marchman und Bates (1994) betonen, dass der frühe Wortschatz ab dem Punkt über eine besonders hohe prädiktive Validität verfügt, wo der expressive Wortschatzumfang eine kritische Größe überstiegen hat. Dieses gelte vor allem für den Verbenwortschatz. Diese Aussage folgt der „,critical mass hypothesis“, welche besagt, das ein Kind erst ab einer bestimmten Anzahl von gesprochenen Wörtern („Bausteinen“) in der Lage ist, Sätze zu bauen und somit in die morpho-syntaktische Entwicklung „einzusteigen“. Das entsprechende Bezugsalter für diese „kritische Masse“ liegt um den 18. bis 21. Lebensmonat, da hier bei den meisten Kindern der Wortschatzspurt einsetzt und es zu einer explosiven Erhöhung des Wortschatzumfanges und ersten Zwei-Wort-Kombinationen kommt.

Paul (2000) hebt hervor, dass insbesondere der expressive Wortschatzumfang in der Entwicklungsphase um die Vollendung des 2. Lebensjahres als zuverlässigster Prädiktor für den späteren Sprachentwicklungsstand belegt sei. Eine große Fülle an Untersuchungen gibt es für das Erhebungsalter von 24 Monaten, da in diesem Alter das Kriterium für „Late Talker“ definiert ist. Pan, Rowe, Spier und Tamis-Lemonda (2003) fanden in ihrer Untersuchung der rezeptiven Sprachleistungen (gemessen mit dem PPVT) von 105 normal entwickelten dreijährigen Kindern aus sozial schwachen Familien eine gute prädiktive Validität $(r=.50)$ des frühen Wortschatzes (mit 2 Jahren) unabhängig von der Datenerhebungsmethode, die auch bei Kontrolle der Intelligenz als möglicher konfundierender Variable erhalten blieb.

Westerlund, Berglund und Eriksson (2006) kommen zu dem Ergebnis, dass der expressive Wortschatzumfang mit 18 Monaten (gemessen mit Parent Report) der beste Prädiktor für das Sprachoutcome mit 3 Jahren ist, erachten hingegen die Sensitivität des verwendeten Parent Report Verfahrens mit 50\% als zu gering für die klinische Diagnose.

Betrachtet man Studien zur prädiktiven Validität des frühen Wortschatzes für das Erhebungsalter von 8 bis 36 Monaten, so werden sehr unterschiedliche Ausprägungen der Zusammenhänge zu späterem Sprachoutcome berichtet, die von .18 bis .81 variieren (vgl. Bornstein \& Haynes, 1998; Fenson et al., 1993; Reese \& Read, 2000). Entscheidend für die Höhe des Zusammenhanges scheint nicht der Erhebungszeitpunkt sondern vielmehr die 
Zeitspanne, für die Vorhersagen gemacht werden, sowie der Tatbestand zu sein, ob die gleichen Erhebungsinstrumente $\mathrm{zu}$ den verschiedenen Testzeitpunkten eingesetzt wurden. Feldman, Campbell, Colborn, Dale, Kurs-Lasky, Paradise et al. (2005) berichten für die Zeitspanne von $24 \mathrm{zu} 36$ Monaten über moderate Korrelationen von 32 für den frühen expressiven Wortschatzumfang (gemessen mit den CDI) und Wortschatz mit drei Jahren gemessen mit dem Peabody Picture Vocabulary Test - Third Edition (PPVT-III; Dunn \& Dunn, 1997). Die Korrelationen für den frühen Wortschatz (CDI) mit späterem Wortschatz-, Satz- und allgemeinem Sprachwert (CDI mit 3 Jahren) fielen mit Werten von .52 bis .70 deutlich höher aus.

Dieses verdeutlicht, dass ein Wechsel der Erhebungsinstrumente im längsschnittlichen Untersuchungsdesign zu substanziell geringeren Korrelationen $(r=.32 \mathrm{im}$ Gegensatz zu $r=$ .52 - .70) zwischen den Sprachmaßen bei identischen Messzeitpunkten führen kann.

Wie gut ist die prädiktive Validität des frühen Wortschatzes für den gestörten Spracherwerb untersucht?

Als Meilenstein in der Sprachentwicklung werden allgemein ein Wortschatzumfang von 50 Wörtern und das Vorhandensein erster Zwei-Wort-Äußerungen in einem Alter von 24 Monaten angenommen (Grimm, 1995a, 1999; Rescorla , 1989; Rescorla, Hadicke-Wiley \& Escarce, 1993, Rescorla et al., 2000; Tamis-Lemonda, Bornstein, Kahana-Kalman, Baumwell \& Cyphers, 1998). Rescorla et al. (1993) bezeichnen die kombinierte Information aus Wortschatzgröße und dem Vorhandensein erster Wortverbindungen als bestes Kriterium, um Risikokinder für eine Sprachentwicklungsstörung zu erfassen. Kinder, die diesen Meilenstein nicht erreichen (13 - 20\% nach Rescorla et al., 1993; 14\% nach Grimm, 1999; 15\% nach Rescorla et al., 2000), werden als „Late Talkers“ bezeichnet und tragen das Risiko für die Entwicklung späterer Sprachentwicklungs- und anderer Entwicklungsstörungen. Auch Berglund und Eriksson (2000) bestätigen, dass den Wortschatzergebnissen eine besonders gute Vorhersagekraft (71 - 88\%) für die Identifikation von Risikokindern im Lebensalter von 25 bis 28 Monaten zukommt. Der „Löwenanteil“ aller Veröffentlichungen zum frühen Wortschatz beschäftigt sich mit dem Phänomen „Late Talker“. Es ist daher auch nicht weiter verwunderlich, dass ebenso bei den Veröffentlichungen zur prädiktiven Validität des frühen Wortschatzes die Mehrzahl der Untersuchungen die Bestimmung der prädiktiven Validität des Kriteriums „Late Talker“ zum Untersuchungsgegenstand hat. Erhoben werden Daten zum weiteren Entwicklungsverlauf dieser Kinder bis ins Jugendalter (Rescorla 2002, 2005), die in 
Beziehung zur Risikodiagnose mit 24 Monaten gesetzt werden. Die Stichproben sind meist kleine Stichproben, da sie nur aus „,klinisch auffälligen“ Kindern bestehen.

Feldman et al. (2005) sowie Dale, Price, Bishop und Plomin (2003) stellen fest, dass der expressive Wortschatzumfang mit 2 Jahren (gemessen mit den CDI) keine ausreichend akkurate Vorhersage des Sprachoutcomes (im klinischen Kontext und Forschungssetting) mit 3 und 4 Jahren liefert.

Bezogen auf den deutschen Sprachraum berichtet Sachse (2005) für den Elternfragebogen für einjährige Kinder: Sprache, Gesten, Feinmotorik - ELFRA-1 (Grimm \& Doil, 2000) in einer Nachuntersuchung mit $n=121$ (monolingual deutschsprachigen Kindern) im Alter von 2 Jahren unter Verwendung des Elternfragebogens für zweijährige Kinder: Sprache und Kommunikation - ELFRA-2 (Grimm \& Doil, 2000) von einer zu niedrigen Risikoidentifikation für spätere Sprachentwicklungsstörungen. Die ELFRA-1 Daten (u. a. auch früher Wortschatzumfang) verfügen über eine Sensitivität von $52 \%$ und Spezifität von 65\%. Der Ratzindex (relative Verbesserung der Trefferquote im Vergleich zum Zufall) ist mit $23 \%$ als unbefriedigend zu bewerten. Somit taugen die auf diese Art erhobenen frühen Daten der Sprachentwicklung (auch der frühe Wortschatzumfang mit 12 Monaten) nicht für die klinische Vorhersage von späteren Sprachentwicklungsstörungen. Bezüglich der Beurteilung der prädiktiven Validität des ELFRA 2 ergab sich für die spätere klinische Diagnose eine Gesamttrefferquote von 90\% (ELFRA $1=63 \%$ ), mit einer Sensitivität von 69\% und Spezifität von 92\%. Die mit dem ELFRA 2 erhobenen Sprachdaten (auch früher Wortschatzumfang) erlauben somit eine ausreichend gute Vorhersage für Sprachentwicklungsstörungen im Alter von 3 Jahren.

Rescorla (2005) berichtet in ihrer Studie zum Entwicklungsverlauf an 28 Late Talkers, dass sich der frühe Wortschatz von Late Talkers mit 24 Monaten als signifikanter Prädiktor für Wortschatz, grammatische Fähigkeiten, Leseverständnis und verbale Merkfähigkeit im Alter von 13 Jahren bewährte.

Insgesamt ist die prädiktive Validität des frühen Wortschatzes für späteres Sprachoutcome für die gestörte Sprachentwicklung vielfältiger dokumentiert als für den regelrechten Spracherwerb und auch wenn normal entwickelte Kinder als Kontrollgruppen im Rahmen der Late Talker Studien untersucht wurden, so sind deren Ergebnisse selten veröffentlicht. Aussagen für den weiteren Entwicklungsverlauf sind eingeschränkt möglich, wenn es um die klinische Vorhersage geht. 
Was ist über die prädiktive Validität des frühen Wortschatzes für späteres Sprachoutcome bei normal entwickelten Kindern bekannt?

Viele Studien zur prädiktiven Validität des frühen Wortschatzes machen Aussagen, die nur für eine relativ kurze Zeitspanne gelten. So werden Wortschatzdaten im Alter von 12 Monaten erhoben und mit Sprachdaten im Alter von 24 Monaten in Beziehung gesetzt (Feldman et al., 2000). Oder die Messzeitpunkte liegen noch enger beisammen (25 bis 28 Monate [Berglund \& Eriksson, 2000] und 20 bis 28 Monate [Bates, Bretherton \& Snyder, 1988]).

Wie ausgeprägt sind die Zusammenhänge für solch ein relativ kurzes Vorhersageintervall von bis zu 12 Monaten?

Fenson et al. (1993) untersuchten an einer Stichprobe von 228 normal entwickelten Mittelschicht-Kindern den frühen Wortschatz (CDI) mit 16 - 24 Monaten $\left(\mathrm{t}_{1}\right)$ und mit 22 - 30 Monaten $\left(\mathrm{t}_{2}\right)$. Die Korrelationen beliefen sich auf .71 (auch bei Kontrolle des Alters).

Bates et al. (1988) fanden signifikante Korrelationen von .60 - .80 für den expressiven Wortschatzumfang gemessen mit Parent Report im Alter von 20 Monaten und Grammatikund Wortschatzleistungen (gemessen mit standardisierten Tests) im Alter von 28 Monaten.

Feldman, Dollaghan, Cambell, Kurs-Lasky, Janosky und Paradise (2000) untersuchten an einer Stichprobe von mehr als 2000 Kindern im durchschnittlichen Alter von 12 Monaten mit den CDI die frühen sprachlichen Fähigkeiten (auch Wortschatz). Die Ergebnisse wurden korreliert mit denen einer Folgeuntersuchung im Alter von 24 Monaten (ebenfalls mit den CDI). Die Korrelationen beliefen sich auf Werte von .18 bis .39.

Pan, Rowe, Spier und Tamis-Lemonda (2003) dokumentieren eine Varianzaufklärung des frühen Wortschatzes (24 Monate, gemessen mit den CDI) am rezeptiven Wortschatz im Alter von 36 Monaten (gemessen mit den CDI) in der Höhe von 25 Prozent, was einer zugrunde liegenden Korrelation von .50 entspricht.

In den meisten Untersuchungen werden keine Regressionsanalysen gerechnet sondern Korrelationsanalysen. Quadrierte Korrelationen können jedoch als Schätzung der aufgeklärten Varianz verstanden werden und die angegebenen Korrelationen erlauben somit Aussagen zur prädiktiven Validität des frühen Wortschatzes. Zusammenfassend lässt sich für das Vorhersageintervall von bis $\mathrm{zu} 12$ Monaten festhalten, dass der frühe expressive Wortschatz überwiegend gute Vorhersagen des späteren Sprachoutcome ermöglicht, denn es bestehen mehrheitlich Korrelationen hoher Effektstärke für dieses Vorhersageintervall. 
Nur wenige Studien untersuchen die Zusammenhänge von frühem Wortschatz und späterem Sprachoutcome über eine längere Zeitspanne.

Bornstein und Haynes (1998) erhoben den frühen Sprachentwicklungsstand bei 184 Kindern im Alter von 20 Monaten mittels Spontansprachproben (Wortschatz und MLU), den Reynell Developmental Language Scales (RDLS; Reynell \& Huntley, 1985; rezeptive und expressive Skala) und dem Early Language Inventory (ELI; Bates et al., 1984) - einem Parent Report Verfahren, welches den Umfang des expressiven Wortschatzes bestimmt. Im Alter von 48 Monaten wurden 154 Kinder mit dem Wechsler Preschool and Primary Scale of Intelligence (WPPSI-R; Wechsler, 1967) untersucht. Für die Korrelationsanalysen wurde das Abschneiden der Kinder im Verbalteil (auch Wortschatz) des WPPSI-R verwandt. Die Korrelation der Spontansprachdaten zu t $\mathrm{t}_{1}$ (20 Monate) mit den Daten zu t $\mathrm{t}_{2}$ (48 Monate) belief sich für die MLU auf .15 und für den Wortschatz .auf 27. Für die Ergebnisse im RDLS konnten Korrelationen von .53 für die rezeptive Skala und von .39 für die expressive Skala berechnet werden. Der frühe Wortschatzumfang gemessen mit dem ELI korrelierte mit .39 mit den späteren Leistungen im Verbalteil des WPPSI-R.

In der Untersuchung von Reese and Read (2000) wurden an einer Stichprobe von 61 normal entwickelten Kindern im Alter von 19 und 25 Monaten u. a. der Wortschatzumfang mit einer für den neuseeländischen Sprachgebrauch adaptierte Version der CDI erhoben. Im Alter von 32 und 40 Monaten wurden die Wortschatzleistungen mit dem Expressive Vocabularity Test (Williams, 1997) und dem Peabody Picture Vocabulary Test - Third Edition (PPVT III; Dunn \& Dunn, 1997) geprüft. Für den Wortschatzumfang im Alter von 19 Monaten konnte eine Korrelation von 81 zum späteren Wortschatzumfang im Alter von 25 Monaten festgestellt werden. Auch die Korrelationen zum Wortschatz im Alter von 32 Monaten und 40 Monaten beliefen sich auf Werte von .46 bis .50.

Walker, Greenwood \& Carta (1994) untersuchten normal entwickelte Kinder ( $\mathrm{n}=32)$ in einer 10-jährigen Längsschnittstudie aufbauend auf den Studien von Hart und Risley (1989) von früher Kindheit (7-36 Monate) bis in die dritte Grundschulklasse. In Beziehung gesetzt wurden die frühen Daten zum sozio-ökonomischen Status (z.B. Beruf, Bildung und Einkommen der Eltern), Intelligenzmaße und expressiver Wortschatzumfang sowie MLU (gemessen anhand von Spontansprachproben im häuslichen Umfeld) mit den späteren Daten im Kindergarten- und Grundschulalter (rezeptive und expressive Sprache gemessen mit dem PPVT (Dunn \& Dunn, 1981) und dem Test of Language Development - 2 (TOLD; Newcomer \& Hammill, 1988). Es ergaben sich signifikante Korrelationen von expressivem Wortschatz (und MLU) mit späteren Sprachleistungen (rezeptiv und expressiv) sowie Lese- 
und Rechtschreibleistungen (1. bis 3. Grundschulklasse). In hierarchischen Regressionsanalysen lieferte der frühe Wortschatzumfang immer einen substanziellen zusätzlichen Varianzaufklärungsbeitrag $\left(\Delta R^{2}=.20\right.$ - .27) für die Vorhersage späterer Sprachleistungen zusätzlich zu frühen sozioökonomischen Maßen. Gleiches galt für die Vorhersage späterer Leseleistungen (1. und 3. Klasse) und Rechtschreibleistungen (1. bis 3. Klasse). Der frühe Wortschatzumfang korrelierte mit der rezeptiven Sprache im Kindergarten (5 Jahre) zu .71. In der ersten Klasse beliefen sich die Korrelationen mit rezeptiver (PPVT) bzw. expressiver (TOLD) Sprache auf .74 und .64. Für die 2. Klasse ergaben sich Korrelationen von .65 und .68 und für die 3. Klasse von .48 und .72. Diese Ergebnisse bestätigten die Autoren in der Annahme, dass der Umfang der früh gesprochenen Sprache im häuslichen Umfeld einer der Schlüsselprädiktoren für spätere Schulleistungen sei.

Bornstein et al. (2004) untersuchten in vier unabhängigen prospektiven Längsschnittstudien Sprachentwicklungsmaße bei Kindern im Alter von 1 bis 7 Jahren. Studie 1 berichtet über drei Messzeitpunkte (2, 3 und 4 Jahre). In Studie 2 wurden die Kinder im Alter von 1;8 und 4;0 Jahren untersucht. Studie 3 enthält die Messzeitpunkte 1;1 sowie 1;8 und 5;10 Jahre und in Studie 4 wurden die Sprachentwicklungsdaten der Kinder im Alter von 4;6 und 6;10 Jahren erhoben. Eine Vielzahl an unterschiedlichen Erhebungsmethoden kam über die Messzeitpunkte zum Einsatz. Ausgewählte Ergebnisse, die bezüglich der Fragestellung und Messzeitpunkte mit der Promotionsstudie vergleichbar sind $(1 ; 9,4 ; 0,5 ; 0$ und 7;10 Jahre) werden folgend dargestellt.

In Studie 1 ergab sich für die mittels Parent report (CDI - WS) erhobenen Sprachdaten mit 2;0 Jahren keine signifikante Korrelation zum Verbalteil des WPPSI-R $(r=.20)$ im Alter von 3 und 4 Jahren. Einschränkend ist anzumerken, dass die Stichprobe aus lediglich 26 Kindern bestand.

In Studie 2 wurden 184 Kinder über eine Altersspanne von 1;8 bis 4;0 Jahren untersucht. Die Korrelationen der frühen Sprachmaße mit den späteren Sprachmaßen betrug bei Verwendung unterschiedlicher Erhebungsmethoden durchschnittlich .29. Die Korrelationen alleinig für den expressiven Wortschatzumfang wurden nicht dokumentiert.

In Studie $3(n=34)$ konnten für die Altersspanne 1;8 bis 5;10 Jahre bezüglich der frühen Wortschatzangaben mittels Parent Report (CDI-WS) und späterer Sprachdaten (erhoben mittels Parent Report und standardisiertem Testen) keine signifikanten Korrelationen errechnet werden. Für die RDLS (rezeptive und expressive Skala) beliefen sich die Korrelationen auf .40. 
Zusammenfassend ist festzuhalten, dass die Ergebnisse für die Vorhersage späteren Sprachoutcomes aus den frühen Wortschatzdaten über eine größere Zeitspanne uneinheitlich sind. Zum Teil wurden die Studien mit kleinen Stichproben ( $n=26$ bis 34$)$ durchgeführt und das Vorliegen signifikanter Korrelationen für den Wortschatz mit späterem Sprachoutcome von $2 ; 0$ bis $4 ; 0$ Jahren bzw. von $1 ; 8$ bis 5;10 Jahren konnte nicht bestätigt werden. Dann wiederum werden Korrelationen über mehrere Jahresspannen von frühem Wortschatz zu späteren Sprachleistungen von .48 bis .72 berichtet. Divergente Ergebnisse in Abhängigkeit vom verwendeten Erhebungsverfahren bei der gleichen Stichprobe werden von Bornstein et al. (2004) berichtet. Es liegen kaum Aussagen für eine größere Stichprobe normal entwickelter Kinder über den Zeitraum frühe Kindheit bis Grundschulalter im angloamerikanischen Raum vor und für den deutschen Sprachraum konnte der aktuellen Forschungsliteratur gar keine entsprechende Untersuchung entnommen werden.

In vorliegender Arbeit soll der Frage nachgegangen werden, ob der frühe expressive Wortschatz signifikante Vorhersagen für das spätere Sprachoutcome bezogen auf unterschiedliche Aspekte von Sprache (Wortschatz, expressive und rezeptive grammatische Fähigkeiten und Satzgedächtnis) im Vorschul- und Grundschulalter erlaubt.

Insgesamt stehen nur wenige Vergleichsstudien zur Verfügung, auf die sich konkret in der Formulierung der psychologischen Hypothesen in dieser Arbeit bezogen werden kann.

Angesichts der Forschungslage ist jedoch für diesen Datensatz mit bedeutsamen Vorhersagen späterer Sprachleistungen aus dem frühen expressiven Wortschatzumfang (erhoben mittels Parent Report) sowohl für die späteren Wortschatzdaten als auch für die grammatische Entwicklung (rezeptiv und expressiv) zu rechnen. Es sind keinerlei Studien bekannt, welche den Zusammenhang von frühem Wortschatz und späteren Satzgedächtnisleistungen untersuchen. Da das Satzgedächtnis jedoch entscheidend von der Verfügbarkeit morphologischer und syntaktischer Regeln sowie vom Sprachverständnis abhängt (Fromm \& Schöler, 1997), werden substanzielle Vorhersagen der späteren Satzgedächtnisleistungen aus den frühen Wortschatzleistungen erwartet.

Folgende psychologische Hypothesen werden abgeleitet:

PH 1: Frühe Wortschatzdaten verfügen über bedeutsame prognostische Validität für spätere Wortschatzleistungen im Vorschul- und Grundschulalter.

PH 2: Frühe Wortschatzdaten verfügen über bedeutsame prognostische Validität für spätere rezeptive grammatische Fähigkeiten im Vorschul- und Grundschulalter. 
PH 3: Frühe Wortschatzdaten verfügen über bedeutsame prognostische Validität für spätere expressive Grammatikleistungen im Vorschul- und Grundschulalter.

PH 4: Frühe Wortschatzdaten verfügen über bedeutsame prognostische Validität für spätere Satzgedächtnisleistungen im Vorschul- und Grundschulalter.

\subsection{Ergebnisse}

Der frühe expressive Wortschatzumfang im Alter von 16 bis 26 Monaten $\left(\mathrm{t}_{0}\right)$ wurde auf seine prädiktive Validität für Sprachleistungen im Alter von 4;0 Jahren ( $\left.\mathrm{t}_{1}\right)$, 5;0 Jahren $\left(\mathrm{t}_{2}\right)$ und 7;10 Jahren $\left(\mathrm{t}_{3}\right)$ im regressionsanalytischen Design geprüft.

Für alle Regressionsanalysen wurde der Prozentrang im ELAN (expressiver Wortschatzumfang gemessen mittels Parent Report zu t $\mathrm{t}_{0}$ ) als Prädiktorvariable verwendet. Als Kriteriumsvariablen für die Vorhersage späterer Wortschatzleistungen fungierten $z t_{1}$ und $t_{2}$ die Rohwerte im AWST 3-6 und $\mathrm{zu} \mathrm{t}_{3}$ die Rohwerte im Untertest „Wortschatz“ aus dem HAWIK III. Für die rezeptiven grammatischen Fähigkeiten wurden die Rohwerte im Untertest „Verstehen von Sätzen“ aus dem SETK 3-5 zu t $\mathrm{t}_{1}$ und $\mathrm{t}_{2}$ sowie die Rohwerte im Untertest „Verstehen grammatischer Strukturformen“ aus dem HSET zu $\mathrm{t}_{3}$ in die Regressionsanalysen eingegeben. Als Kriteriumsvariablen für die Berechnungen zur Vorhersage expressiver grammatischer Leistungen fungierten die Rohwerte aus dem Untertest „Morphologische Regelbildung“ (SETK 3-5) zu $t_{1}$ und $t_{2}$ sowie die Rohwerte aus dem Untertest „Plural-Singular-Bildung“ (HSET) zu $t_{3}$. Für die Vorhersage der Satzgedächtnisleistungen gingen als Kriteriumsvariablen die Rohwerte aus dem Untertest „Satzgedächtnis“ (SETK 3-5) zu $t_{1}$ und $t_{2}$ sowie die Rohwerte aus dem Untertest „Imitation grammatischer Strukturformen“ (HSET) zu t $\mathrm{t}_{3}$ in die Berechnungen ein. Um dem Alterseffekt bezogen auf das Erhebungsalter des frühen Wortschatzes (Zeitspanne von 10 Monaten zu $\mathrm{t}_{0}$ ) gerecht zu werden, wurden hierarchische Regressionsanalysen gerechnet, in welche im ersten Block (schrittweise) das Alter der Kinder als Prädiktorvariable eingegeben wurde und erst im zweiten Block der Prozentrang im ELAN.

Exemplarisch werden die empirischen Vorhersagen in Bezug auf die Sprachkomponente Wortschatz aufgestellt:

EV 1: Es werden signifikante Varianzaufklärungen für den Prozentrang im ELAN als Prädiktorvariable und die Rohsumme im AWST 3-6 als Kriteriumsvariable zu t $\mathrm{t}_{1}$ erwartet.

EV 2: Es werden signifikante Varianzaufklärungen für den Prozentrang im ELAN als

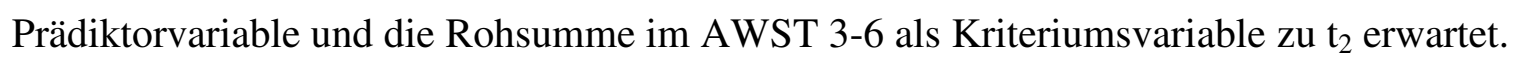


EV 3: Es werden signifikante Varianzaufklärungen für den Prozentrang im ELAN als Prädiktorvariable und die Rohsumme im Untertest Wortschatz aus dem HAWIK III als Kriteriumsvariable zu t 3 erwartet.

Tabelle 10 stellt die Ergebnisse der Regressionsanalysen dar. 
Tabelle 10

Vorhersagen späterer Sprachleistungen von $t_{1}$ bis $t_{3}$ aus dem frühen Wortschatz

$\boldsymbol{R}$

Wortschatz

$\mathbf{t}_{1}$

$\mathbf{t}_{2}$

$\mathbf{t}_{3}$
.382

.515

.276
$\boldsymbol{R}^{2}$ korr

.122

.251

.058

df $\quad F$

$\boldsymbol{F}$

\section{Expressive grammatische Leistungen}

\begin{tabular}{|c|c|c|c|c|c|}
\hline $\mathbf{t}_{1}$ (3-jährige) & .090 & -.033 & 25 & 0.197 & .661 \\
\hline (4-jährige) & .658 & .413 & 30 & 22.15 & $.000 * *$ \\
\hline $\mathbf{t}_{2}$ & .376 & .125 & 53 & 8.578 & $.005^{* *}$ \\
\hline $\mathbf{t}_{3}$ & .300 & .072 & 52 & 5.046 & $.029 *$ \\
\hline
\end{tabular}

Rezeptive grammatische Leistungen

\begin{tabular}{|c|c|c|c|c|c|}
\hline $\mathbf{t}_{1}$ (3-jährige) & .209 & .004 & 25 & 1.099 & .305 \\
\hline (4-jährige) & .463 & .187 & 30 & 7.9 & $.009 * *$ \\
\hline $\mathbf{t}_{2}$ & .364 & .116 & 53 & 7.967 & $.007 * *$ \\
\hline $\mathbf{t}_{\mathbf{3}}$ & .215 & .028 & 52 & 2.481 & .121 \\
\hline
\end{tabular}

\section{Satzgedächtnis}

$\begin{array}{llllll}\mathbf{t}_{\mathbf{1}} & .424 & .151 & 30 & 6.346 & .018 * \\ \mathbf{t}_{\mathbf{2}} & .432 & .168 & 44 & 9.855 & .003 * * \\ \mathbf{t}_{\mathbf{3}} & .384 & .131 & 52 & 8.844 & .004 * *\end{array}$

Anmerkungen. $\mathrm{t}_{1}-\mathrm{t}_{3}=$ Messzeitpunkt 1 bis 3, $R=$ Korrelationskoeffizient, $R^{2}{ }_{\text {korr }}=$ Korrigiertes Bestimmtheitsmaß, $d f=$ Freiheitsgrade, $F=$ F-Wert, $p=$ Fehlerwahrscheinlichkeit, $*=\mathrm{p}<$ $.05, * *=\mathrm{p}<.01$ 
Als Maß für die Effektstärken der Vorhersagen wurde das korrigierte Bestimmtheitsmaß $\left(R_{\text {korrigiert }}^{2}\right.$ dem unkorrigierten $R^{2}$ vorgezogen, um der Überschätzung des Varianzaufklärungbeitrages auf Grundlage einer relativ kleinen Stichprobe vorzubeugen. Das unkorrigierte $R^{2}$ tendiert zur Überschätzung des goodness of fit von Daten, die an kleineren Stichproben erhoben wurden.

Für die Vorhersage der späteren Wortschatzleistungen ließen sich über alle Messzeitpunkte signifikante Varianzaufklärungen berechnen. So ergab sich $\mathrm{zu} \mathrm{t}_{1}$ ein korrigiertes $R^{2}$ von $.122(F=6.14 ; p=.02)$. Für die Vorhersage $\mathrm{zu} \mathrm{t}_{2}$ belief sich $R_{\text {korrigiert }}^{2}$ auf


Die empirischen Vorhersagen hinsichtlich der weiteren Wortschatzentwicklung gelten für alle Messzeitpunkte als zutreffend.

Die Vorhersage der Leistungen im Untertest „Morphologische Regelbildung“ (expressive grammatische Leistungen) $\mathrm{zu}_{1}$ ergab für die dreijährigen Kinder keine signifikante Varianzaufklärung $\left(R_{\text {korrigiert }}^{2}=-.033[F=.197 ; p=.66]\right)$. Für die vierjährigen Kinder belief

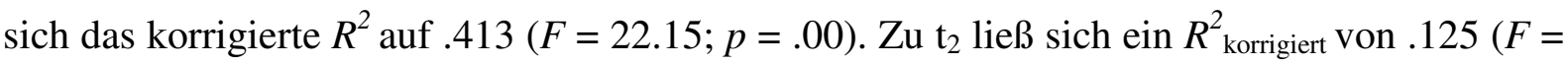
$8.58 ; p=.005)$ und zu t 3 ein $R_{\text {korrigiert }}^{2} .072(F=5.05 ; p=.029)$ berechnen. Die empirischen Vorhersagen zur morphologischen Regelbildung sind mit Ausnahme der Vorhersage für die Stichprobe der dreijährigen Kinder zu $\mathrm{t}_{1}$ zutreffend.

Auch der Varianzaufklärungsbeitrag für die Leistungen im Untertest „Verstehen von Sätzen“ (rezeptive grammatische Leistungen) $\mathrm{zu} \mathrm{t}_{1}$ für die Dreijährigen war nicht signifikant $\left(R_{\text {korrigiert }}^{2}=.004[F=1.01 ; p=.30]\right)$; hingegen zeigte sich für die vierjährigen Kinder ein $R^{2}{ }_{\text {korrigiert }}$ von .187 $(F=7.9 ; p=.01)$. Das korrigierte $R^{2}$ für die Prädiktion der Verstehensleistungen zu $\mathrm{t}_{2}$ erreichte einen Wert von .116 $(F=7.97 ; p=.01)$. Für $\mathrm{t}_{3}$ ergab sich keine signifikante Vorhersage $\left(R^{2}{ }_{\text {korrigiert }}=.028[F=2.48 ; p=.12]\right)$.

Als zutreffend erwiesen sich die empirischen Vorhersagen für die rezeptiven grammatischen Fähigkeiten der vierjährigen Kinder zu $t_{1}$ und für die gesamte Stichprobe zu $t_{2}$. Nicht zutreffend waren die empirischen Vorhersagen bzgl. des Sprachverstehens für die dreijährigen Kinder zu $t_{1}$ und für die Gesamtstichprobe zu $t_{3}$.

Der Untertest Satzgedächtnis zu $\mathrm{t}_{1}$ war nur für die vierjährigen Kinder vorgesehen. Für diese ergab sich ein korrigiertes $R^{2}$ von $.151(F=6.35 ; p=.018)$. $\mathrm{Zu} \mathrm{t}_{2}$ belief sich $R^{2}{ }_{\text {korrigiert }}$ auf $.168(F=9.86 ; p=.00)$ und $\mathrm{zu} \mathrm{t}_{3}$ konnte ein korrigiertes $R^{2}$ von $.131(F=8.84 ; \mathrm{p}=.00)$ errechnet werden. Die empirischen Vorhersagen hinsichtlich des Satzgedächtnisses konnten ausnahmslos bestätigt werden. 
Für alle Berechnungen konnte die Variable Alter im ersten Block der Regressionsanalysen keinen substanziellen Aufklärungsbeitrag leisten. Bei Verzicht auf die Blockeingabe erzielte die Variable Alter in schrittweisen Regressionsanalysen nur für das Satzgedächtnis zu $t_{2}$ und $\mathrm{zu} \mathrm{t}_{3}$ im 2. Schritt eine signifikante Erhöhung der Varianzaufklärung. So ergab sich $\mathrm{zu}_{2}$ ein $R^{2}$ von $.143\left(R_{\text {korrigiert }}^{2}=.311 ; F=10.91 ; p=.000\right)$ und zu t $\mathrm{t}_{3}$ belief sich $R^{2}$ auf $.105\left(R_{\text {korrigiert }}^{2}\right.$ $=.236 ; F=9.03 ; p=.000)$.

\subsection{Diskussion}

Die Psychologische Hypothese 1 (Frühe Wortschatzdaten verfügen über bedeutsame prognostische Validität für spätere Wortschatzleistungen im Vorschul- und Grundschulalter.) sowie die Psychologische Hypothese 4 (Frühe Wortschatzdaten verfügen über bedeutsame prognostische Validität für spätere Satzgedächtnisleistungen im Vorschul- und Grundschulalter.) konnten an vorliegendem Datensatz bei strenger Prüfung bestätigt werden.

Die Psychologischen Hypothesen 2 und 3 (Frühe Wortschatzdaten verfügen über bedeutsame prognostische Validität für spätere rezeptive und expressive grammatische Fähigkeiten im Vorschul- und Grundschulalter.) konnten bei strenger Prüfung (konjunktive Verknüpfung aller empirischen Vorhersagen) im Rahmen dieser Arbeit nicht bestätigt werden, da für bestimmte Untersuchungszeitpunkte bzw. Teilstichproben keine signifikanten Varianzaufklärungsbeiträge des frühen Wortschatzes ermittelt werden konnten.

Einschränkend muss erwähnt werden, dass sich für die expressiven grammatischen Fähigkeiten lediglich bzgl. der Stichprobe der dreijährigen Kinder keine signifikante Varianzaufklärung durch den frühen Wortschatz ergab (wohl aber für die Stichprobe der vierjährigen Kinder). Diese Teilstichprobe bestand aus 26 Kindern und musste andere Aufgaben im Untertest „Morphologische Regelbildung“ lösen als die vierjährigen Kinder. So mussten die dreijährigen Kinder im Gegensatz zu den vierjährigen keine Pluralbildung für Kunstwörter vornehmen. Die Pluralbildung bei Kunstwörtern könnte jedoch ein in höherem Maß zuverlässiger und valider Marker für die expressiven Grammatikleistungen sein als die Pluralbildung bei alltagstypischen Wörtern. Der negative Wert für das Bestimmtheitsmaß $R^{2}$ korrigiert deutet hierbei an, dass bei einem sehr geringen Korrelationskoeffizienten von .09 und unkorrigierten Bestimmtheitsmaß $\left(R^{2}=.008\right)$ der Rechenalgorithmus zur Korrektur der Überschätzung des Varianzaufklärungsbeitrages nicht mehr greift und ein rechnerisch scheinbar negatives unlogisches Ergebnis auswirft. 
Das Ergebnis für die dreijährigen Kinder muss im Hinblick auf die geringe Größe der Stichprobe und die Operationalisierung des Konzeptes expressive grammatische Leistungen relativierend für den Rückschluss auf die psychologische Hypothese betrachtet werden.

Gleiches gilt für die Vorhersage rezeptiver Grammatikleistungen bzgl. des Erhebungszeitpunktes $\mathrm{t}_{1}$. Auch hier konnten für die Stichprobe der dreijährigen Kinder $(n=$ 26) im Gegensatz zu den vierjährigen Kindern bei anderen Aufgaben im Untertest „Verstehen von Sätzen“ keine signifikanten Varianzaufklärungen für den frühen Wortschatz dokumentiert werden. Die dreijährigen Kinder mussten im Vergleich zu den vierjährigen Kindern im Untertest „Verstehen von Sätzen“ weniger Aufträge in Spielhandlungen umsetzten, dafür aber zu vorgegebenen Sätzen die entsprechenden bildlichen Darstellungen zeigen. Hier mögen zum einen visuelle Wahrnehmungsaspekte (z.B. Über- bzw. Unterforderung durch die Anzahl der ablenkenden Bilder) mit sprachlichen Leistungen konfundiert sein und zum anderen die Auftragssituation für eine Spielhandlung näher an der Abbildung rezeptiver grammatischer Leistungen im kindlichen Alltag liegen als die Aufgabe passende Bilder (aus einer Menge von Ablenkern) zu gesprochenen Sätzen zu zeigen. Folglich könnte der Untertest „Verstehen von Sätzen“ die rezeptiven grammatische Leistungen weniger valide und reliabel für dreijährige Kinder erfassen als für vierjährige Kinder.

Auch $\mathrm{zu} \mathrm{t}_{3}$ ließen sich keine signifikanten Vorhersagen aus dem frühen Wortschatz für die rezeptiven grammatischen Fähigkeiten machen. Da die Regressionsanalysen zu diesem Zeitpunkt für die gesamte Stichprobe von 53 Kindern berechnet wurden, kann das Ergebnis nicht durch die besonders kleine Stichprobe einschränkend bewertet werden. Insgesamt sind die Effektmaße für die Vorhersagen über die Zeitspanne von $\mathrm{t}_{0} \mathrm{zu} \mathrm{t}_{3}$ recht niedrig und für das Merkmal Sprache, welches sich als kein hochstabiles Merkmal über diese Zeitspanne von 6 Jahren gezeigt hat (vgl. Kap. 3), überrascht es nicht, dass keine signifikanten Vorhersagen aus einer Sprachkomponente für eine andere (Wortschatz $\mathrm{zu}$ rezeptiven grammatischen Fähigkeiten) möglich waren. Zudem war die Varianzaufklärung aus dem frühen Wortschatz für die rezeptiven grammatischen Fähigkeiten schon $\mathrm{zu} \quad \mathrm{t}_{2}$ mit $12 \%$ relativ gering (insbesondere im Unterschied zur Komponente Wortschatz mit 25\%).

Dass zu keinem Messzeitpunkt und für keine der unterschiedlichen Sprachkomponenten das Alter der Kinder zu $\mathrm{t}_{0}$ trotz der großen Altersspanne von 10 Monaten einen substanziellen Varianzaufklärungsbeitrag für späteres Sprachoutcome im ersten Schritt leistete, weist darauf hin, dass der frühe Wortschatz altersunabhängig spätere sprachliche Fähigkeiten vorhersagt. Zusätzliche Varianzaufklärung leistete das Alter der Kinder $\mathrm{zu} \mathrm{t}_{0}$ lediglich für das 
Satzgedächtnis zu $t_{2}$ und $t_{3}$. Dieses unterstützt die Annahme, dass Satzgedächtnis in besonderem Maße von kognitiven (reifungsbedingten) Faktoren abhängt. Dieses gilt für die anderen untersuchten Aspekte von Sprache nicht.

Von großem Interesse vor allem mit Hinblick auf die praktisch-klinische Relevanz der Ergebnisse ist die Frage, wie gut der frühe Wortschatz spätere Sprachleistungen vorherzusagen vermag. Dieses lässt sich ablesen an der Höhe des Bestimmtheitsmaßes $\left(R_{\text {korrigiert }}^{2}\right.$, welches den prozentualen Varianzaufklärungsbeitrag einer Prädiktorvariable angibt. Aber wie gut ist denn eine Varianzaufklärung von z.B. 25\% über eine Zeitspanne von 38 Monaten. Üblich ist der Bezug auf die Effektstärken der zugrunde liegenden Korrelationen in Anlehnung an die Konvention von Cohen (1988; vgl. Kap. 3). Um darüber hinaus den Vergleich mit den im theoretischen Hintergrund berichteten Ergebnissen anderer Studien zu erleichtern wird im Folgenden überwiegend auf Korrelationen Bezug genommen.

Für die Güte der Vorhersagen wäre zu erwarten, dass diese mit größer werdender Zeitspanne abnimmt (vgl. Kap. 3). Der Einsatz unterschiedlicher Erhebungsverfahren zu t $t_{1}$ und $t_{2}$ im Vergleich zu $t_{3}$ vermindert hingegen die Vergleichbarkeit der Ergebnisse über den kompletten Zeitstrahl der Längsschnittuntersuchung. Nur für die sprachlichen Aspekte expressive und rezeptive grammatische Fähigkeiten (erhoben mit den Untertests VS und MR) zeigt sich diese Tendenz der abnehmenden Vorhersagegüte von kurzem zu längerem Vorhersageintervall in den Daten. Für den Wortschatz gilt, dass die Varianzaufklärung von $\mathrm{t}_{0}$ $\mathrm{zu} \mathrm{t}_{2}$ höher ist als die von $\mathrm{t}_{0} \mathrm{zu}_{\mathrm{t}}$. Dieses lässt sich dadurch erklären, dass $\mathrm{zu} \mathrm{t}_{1}$ nur 38 von 57 Kindern den AWST 3-6 innerhalb einer belastenden Gesamttestung durchführten und zudem der AWST 3-6 vor allem für junge Kinder durch die wenig ansprechenden bildlichen Darstellung z. T. veralteter und schwer zu erkennender Objekte als wenig motivierend einzustufen ist. Bezogen auf die Ergebnisse im Satzgedächtnis unterscheidet sich das Ausmaß der Varianzaufklärung von $\mathrm{t}_{0} \mathrm{zu} \quad \mathrm{t}_{1}$ im Vergleich zur Zeitspanne $\mathrm{t}_{0} \mathrm{zu}_{2} \mathrm{t}_{2}$ nur minimal. Zu bedenken ist, dass $\mathrm{zu} \mathrm{t}_{1}$ nur für die Stichprobe der 4jährigen Kinder $(n=31)$ das Satzgedächtnis erhoben wurde.

Wie gut ist die Vorhersage sprachlicher Leistungen $\mathrm{zu} \mathrm{t}_{1}$ aus den frühen Wortschatzdaten also für eine Zeitspanne von mehr als 2 Jahren?

Die prozentuale Varianzaufklärung beläuft sich auf Werte von 12 bis $41 \%$. Signifikante Korrelationen von .38 bis .66 wurden errechnet. Dieses entspricht einer mittleren Korrelation 
von .48. Den Konventionen von Cohen (1988) folgend sind diese Korrelationen zwischen Sprachoutcome und frühen Wortschatzdaten über die Zeitspanne von 26 Monaten als mittel bis hoch zu bewerten. Der frühe Wortschatz erlaubt folglich für das Vorhersageintervall von 26 Monaten moderate bis gute Vorhersagen für späteres Sprachoutcome. Gut vergleichen lassen sich die Ergebnisse mit denen von Bornstein und Haynes (1998), die für eine Stichprobe von 154 Kindern für die Zeitspanne von 20 bis 48 Monaten für Parent Report und standardisiertes Testen Korrelationen von .39 bis $.53 \mathrm{zu}$ späterem Sprachoutcome fanden. Reese und Read (2000) dokumentierten für eine ähnlich große Stichprobe von 61 Kindern Korrelationen zwischen frühem Wortschatz mit 19 Monaten und späteren Sprachleistungen mit 40 Monaten von .46 bis .50 . Dieses spricht für die Zuverlässigkeit und Gültigkeit der Aussagen, die auf Grundlage der Stichprobe und des Untersuchungsdesigns dieser Dissertation zur prädiktiven Validität des frühen Wortschatzes für späteres Sprachoutcome gemacht werden. Darüber hinaus bestärken die Ergebnisse die Erwartung, dass die für den angloamerikanischen Sprachraum erforschten Ergebnisse auch für den deutschen Sprachraum Gültigkeit beanspruchen dürfen.

$\mathrm{Zu} \quad \mathrm{t}_{1}$ zeigen die expressiven grammatischen Leistungen (operationalisiert über die morphologische Regelbildung) den höchsten korrelativen Zusammenhang zu den frühen Wortschatzdaten $(r=.66)$. Dieses ist nicht unerwartet, wenn man in Betracht zieht, dass die aktuelle Forschungsliteratur den besonders engen Zusammenhang zwischen Wortschatz und Grammatik übereinstimmend betont (vgl. Conboy \& Thal, 2006) und zudem offenkundig ist, dass die Pluralbildung am Baustein Wort, insbesondere von selbigem abhängt, ja ohne diesen Baustein gar nicht vollzogen werden kann. Allerdings müssten für die Zusammenhänge innerhalb der gleichen Sprachkomponente (in diesem Fall also Wortschatz) mindestens ähnlich hohe korrelative Zusammenhänge bestehen (vgl. Kap. 3: homotype vs. heterotype Stabilität). $\mathrm{Zu} \mathrm{t}_{1}$ wurde der Wortschatz jedoch nicht nur mit einer anderen Erhebungsart (standardisiertes Testen vs. Parent Report) als zu t $\mathrm{t}_{0}$ sondern auch mit einem für junge Kinder wenig motivierenden Verfahren erhoben, was die geringeren Korrelationen erklären hilft.

Für das Zeitintervall von $\mathrm{t}_{0} \quad \mathrm{zu} \quad \mathrm{t}_{2} \quad(38$ Monate) erzielte der frühe Wortschatz Varianzaufklärungen in Höhe von 12 bis $17 \%$, was Korrelationen von .36 bis .52 entspricht. Die mittlere Korrelation von .42 ist gut mit den Ergebnissen von Bornstein et al. (2004) zu vergleichen, die für die Zeitspanne von 1;8 Jahren bis 5;10 Jahren $(n=34)$ mittlere Korrelationen für die RDLS von .40 berichten. Die größte Varianzaufklärung für diese Zeitspanne konnte erwartungskonform innerhalb des gleichen sprachlichen 
Untersuchungsaspektes sprich für den Wortschatz gefunden werden. Dieses bekräftigt die Annahme, dass die geringe Varianzaufklärung für den Wortschatz $\mathrm{zu} \mathrm{t}_{1}$ durch die kleine Stichprobe sowie die wenig motivierende Art des AWST 3- 6 für kleine Kinder hervorgerufen wurde. Die Ergebnisse sprechen für Vorhersagen mittlerer Effektgröße bzgl. des Sprachoutcomes im Alter von 5 Jahren aus den frühen Wortschatzdaten.

Das Zeitintervall von $t_{0}$ bis $t_{3}$ beläuft sich auf 6 Jahre. Für diese große Zeitspanne erreichten die signifikanten Varianzaufklärungsbeiträge Werte von 6 bis 13\%, wobei für die rezeptiven grammatischen Fähigkeiten keine signifikanten Vorhersagen vorgenommen werden konnten. Die entsprechenden Korrelationen beliefen sich auf Werte von .28 bis .38. Dementsprechend kommt dem frühen expressiven Wortschatz für die Vorhersage von Sprachleistungen im Grundschulalter nur eine niedrige bis mittlere prädiktive Validität zu. Die Einordnung der Ergebnisse im Vergleich zu anderen Studien ist nicht möglich. Die Studie von Weindrich et al. (2005) kann aufgrund des Bestehens seiner Untersuchungsstichprobe aus Risikokindern nicht herangezogen werden und die Untersuchung von Walker et al. (1994) wird kritisch betrachtet, da sie an nur 32 Kindern Daten erhebt, das Erhebungsalter des frühen Wortschatzes von 7 bis 36 Monaten eine enorme Spannweite beträgt und schon die Ergebnisse für das Vorhersageintervall frühe Kindheit bis ins Kindergartenalter von 5 Jahren mit .71 Werte dokumentiert, die mit dem aktuellen Forschungsstand nicht konform gehen.

Interessant ist, dass $\mathrm{zu} \mathrm{t}_{3}$ der tendenziell höchste korrelative Zusammenhang zwischen frühem Wortschatz und Satzgedächtnis bestand und die Höhe des Zusammenhanges dieser beiden Aspekte von Sprache sich von $t_{2} z u t_{3}$ nur leicht verringerte. Dieses könnte wiederum als Hinweis dafür gewertet werden, dass Satzgedächtnis in besonderem Maße von kognitiven Faktoren bedingt ist, denn die kognitive Entwicklung präsentiert sich für das Schulalter als relativ stabiles Merkmal.

Das Berichten von tendenziell höheren Korrelationen des frühen Wortschatzes zu bestimmten Sprachaspekten über die Zeit wirft innerhalb der Diskussion der Ergebnisse die Frage auf, ob der frühe expressive Wortschatz einen bestimmten Aspekt von Sprache besonders gut vorhersagt. Geprüft wurden daraufhin die Korrelationsdifferenzen für die Korrelationen der frühen Wortschatzdaten mit den Sprachleistungen $\mathrm{zu}_{2}$ und $\mathrm{t}_{3}$, da zu diesen Erhebungszeitpunkten keine Teilung der Stichprobe erfolgt war. Es ergaben sich keine signifikanten Korrelationsdifferenzen. Somit ist die Aussage, dass der frühe Wortschatz bestimmte Aspekte von Sprache substanziell besser vorhersagt als andere, auf Grundlage vorliegender Datenbasis nicht zulässig. Dieses spricht für die vergleichbar hohe Relevanz der 
im frühen Wortschatz repräsentierten Informationen für alle untersuchten sprachlichen Aspekte über die Zeit.

Die Beantwortung eben beschriebener zusätzlicher Frage ist nur eine von vielen Möglichkeiten, auf Grundlage vorliegender Datenbasis weitere Forschungsfragen aufzuwerfen und zu beantworten.

Als Fazit ergibt sich im Rückschluss auf die formulierten psychologischen Hypothesen, dass der frühe expressive Wortschatz bis zu einem Alter von 5 Jahren substanzielle Vorhersagen für das Sprachoutcome bezogen auf alle Sprachaspekte machen kann. Für das Grundschulalter sind für die Sprachkomponenten Wortschatz, expressive grammatische Fähigkeiten und Satzgedächtnis bedeutsame Vorhersagen möglich, nicht jedoch für rezeptive grammatische Fähigkeiten. Die Güte der Vorhersagen nimmt über die Zeit ab und ist insbesondere für das Grundschulalter als niedrig einzustufen. Die Ergebnisse stimmen mit den Aussagen zur Stabilität von Sprache (siehe Kap. 3) überein.

Die moderaten bis hohen Varianzaufklärungsbeiträge des frühen Wortschatzes für das Sprachoutcome im Vorschulalter sprechen für die engmaschige Kontrolle von Late Talkers und das Einleiten von geeigneten Interventionsmaßnahmen in der frühen Kindheit. Die relativ niedrigen Varianzaufklärungen des frühen Wortschatzes für späteres Sprachoutcome normal entwickelter Kinder über eine große Zeitspanne von 6 Jahren fordern hingegen dazu auf, von einer übertriebenen Beunruhigung der Eltern von Late Talkers allein auf Grundlage der frühen Wortschatzdaten mit Hinblick auf das Schulalter abzusehen; denn eindeutige langfristige Aussagen zum Aufholen bzw. Persistieren der Defizite sind auch aufgrund der Forschungslage zum regelrechten Spracherwerb nicht wahrscheinlich.

Generell sollte von Vorhersagen späterer Sprachleistungen im Grundschulalter allein aus den frühen Wortschatzdaten abgesehen werden.

Stärke vorliegender Untersuchung ist insbesondere, dass sie die prädiktive Validität des frühen Wortschatzes über eine so große Zeitspanne von 22 bis 94 Monaten an einer (für ein Längsschnittprojekt) relativ großen Stichprobe untersucht hat und Aussagen für den deutschen Sprachraum ermöglicht, die bisher nur aus den wenigen Studien im angloamerikanischen Raum abgeleitet wurden. Zudem bieten die Ergebnisse für die kürzeren besser beforschten Zeitspannen, welche gut mit denen der aktuellen Forschung übereinstimmen, die Möglichkeit auch anderen wenig oder gar nicht beforschten Fragen (vgl. Kap. 5, 6 und 7) zum Spracherwerb mit begründetem Vertrauen in vorliegende Datenbasis nachzugehen. 
Die Diskussion der Ergebnisse zu den Vorhersagen späteren Sprachoutcomes aus dem frühen Wortschatz sowie die Ergebnisse zur Stabilität von Sprache (vgl. Kap.3) unterstreichen, dass Sprache ein hochkomplexes multifaktoriell bedingtes Merkmal ist, dessen Vorhersage nur in Beachtung aller möglichen Einflussfaktoren (genetische wie Umweltfaktoren) und im offenen Wissensdiskurs benachbarter Forschungsdisziplinen wie z.B. Linguistik und Psychologie verbessert werden könnte. Fakt ist, dass die Güte der Vorhersagen späterer Sprachleistungen durch die Stabilität des Merkmals Sprache begrenzt ist.

Die hohe Bedeutung des Merkmals Sprache für die weiteren Entwicklungschancen eines Kindes fordert auf, nach weiteren Faktoren und Maßen innerhalb der frühen Sprachentwicklung und verwandten Entwicklungsbereichen $\mathrm{zu}$ forschen, welche die Vorhersage des frühen Wortschatzes für späteres Sprachoutcome substanziell verbessern können (vgl. Kap. 5 und 7) bzw. bessere Vorhersagen als der frühe Wortschatz erlauben. 


\section{Frühe grammatische Fähigkeiten - Verbesserung der Vorhersage späteren Sprachoutcomes}

\subsection{Theoretischer Hintergrund}

Margaret Morse Nice (1925) schlug vor, dass ,,average sentence length may well prove to be the most important single criterion for judging a child`s progress in the attainment of adult language” (p. 378). Die durchschnittliche Äußerungslänge (im Englischen „Mean Length of Utterance“ [MLU] genannt) spielte eine entscheidende Rolle in Roger Brown`s Stufenmodell der Grammatikentwicklung (1973) und ist seitdem das Standardmaß für grammatischen Fortschritt im angloamerikanischen Sprachraum. In den letzten drei Jahrzehnten wurden die Eigenschaften der MLU als abhängige und unabhängige Variable vielfältig untersucht. Die MLU ist ein globales und sehr nützliches Maß für den grammatischen Fortschritt kleiner Kinder (Szagun, 2001). Sie kann in Worten und Morphemen gemessen werden, wobei als Morphem die kleinste (bedeutungstragende) sprachliche Einheit auf der Wortebene bezeichnet wird. Viele Studien verwenden die durchschnittliche Äußerungslänge in Morphemen mit dem Hinweis, dass diese das vermutlich genauere Maß für spätere Grammatikentwicklung sei. In aktuellen Untersuchungen konnte sich die MLU in Worten hingegen als gleichermaßen effektives Maß für die Vorhersage späterer Sprachleistungen bewähren (siehe Parker, 2005). So fanden z.B. Rice, Redmond und Hoffman (2006) in ihrer Untersuchung auf der Basis eines längs- und querschnittlichen Untersuchungsdesigns (Altersspanne 3 bis 10 Jahre) korrelative Zusammenhänge zwischen der MLU gemessen in Morphemen und der MLU gemessen in Worten von .98. Diese hohen Korrelationen galten sowohl für Kinder mit spezifischer Sprachentwicklungsstörung als auch für sprachlich normal entwickelte Kinder. Die MLU ist ein rein quantitatives Maß der Grammatikentwicklung, zeigt aber den Erwerb neuer grammatischer Strukturen (sowohl morphologischer wie auch syntaktischer) an. Sie gilt als zuverlässiger Indikator verbaler Komplexität und grammatischer Entwicklung in der Kinder- und Erwachsenensprache (Bates \& Carnevale, 1993; Brown, 1973). Dale (1990) schätzt die Reliabilität der durchschnittlichen Äußerungslänge im Kindesalter auf .80 und Blake, Quartaro und Onorati (1993) geben Werte von .69 - .82 für die Stabilität der MLU im frühen Kindesalter an. Szagun und Steinbrink (2004) heben hervor, dass die mittlerer MLU im Alter von 1;6 bis 2;2 Jahren langsam zunimmt. Ein deutlicher Anstieg der mittleren MLU erfolgt jedoch ab 2;3 Jahren und die höchste Variabilität konnte für das Alter 2;4 bis 2;6 Jahre dokumentiert werden. Vergleichsweise schneller steigt der expressive Gesamtwortschatz im Alter von 1;6 bis 2;6 Jahren an. 
Etablierte Verfahren zur Erfassung der frühen Sprachentwicklung wie das Language Development Survey (LDS; Rescorla, 1989) und die CDI erfassen die MLU als quantitatives Maß für die Bestimmung der grammatischen Entwicklung; denn für die Gruppe der Late Talker konnte nachgewiesen werden, dass sie sowohl im Diagnosealter als auch zu späteren Untersuchungszeitpunkten signifikant geringere Werte für die MLU aufwiesen als normal entwickelte Kinder (Rescorla, 2000).

Rice et al. (2006) fanden in ihren Untersuchungen an Kindern mit spezifischer Sprachstörung und Kontrollgruppen-Kindern (gematched nach MLU und Alter) hohe Übereinstimmungen der MLU mit anderen unabhängigen Indikatoren für Satzkomplexität (z.B. Developmental Sentence Scoring [DSS]). Darüber hinaus stellten sie fest, dass die MLU als reliabler und valider Index für Satzkomplexität Gültigkeit auch bei der Auspartialisierung der Variablen „Alter“ und „Zuwachs im rezeptiven Wortschatz“ bewahrt. Hierbei lieferte die Auswertung der längsschnittlich erhobenen Daten den stärksten Beweis für die robuste Reliabilität und Validität der MLU sowohl für sprachentwicklungsgestörte als auch normal entwickelte Kinder. Die Autoren fassen zusammen, dass die MLU gut geeignet sei, die allgemeine Sprachentwicklung von Kindern abzubilden, als Gruppierungsvariable (Kinder mit Sprachentwicklungsstörung vs. Kontrollgruppe) für Untersuchungsdesigns zum gestörten Spracherwerb zu fungieren und Einsichten in die Ähnlichkeiten und Besonderheiten des Entwicklungsverlaufs von Kindern mit spezifischer Sprachstörung zu ermöglichen. Gleichzeitig weisen Rice et al. (2006) darauf hin, dass es notwendig sei besondere Sorgfalt bei der Aggregierung der Äußerungsbeispiele sowie der Datencodierung und -analyse walten zu lassen. So sollte z.B. eine ausreichende Anzahl an Äußerungen zur Auswertung vorliegen und die Äußerungen des Kindes sollten nicht durch zu viele Fragen des Versuchsleiters beeinflusst werden.

Bisher gibt es kaum dokumentierte Studien, die der Forschungsfrage nachgehen, ob sich die Vorhersage späterer Sprachleistungen im Vorschul- und Grundschulalter aus den frühen Wortschatzdaten durch das Hinzunehmen der MLU als zweiter Prädiktorvariable verbessern lassen. In der Studie von Berglund und Eriksson (2000) wird die zusätzliche Varianzaufklärung der maximalen Äußerungslänge (Maximum Length of Utterance; MaxLU) für die Vorhersage der Sprachleistungen für das Zeitintervall von 25 bis 28 Monaten untersucht. Alle Vorhersagen bezogen sich auf die Störungsvorhersage. Ziel der Untersuchung war es die Güte der Vorhersagen für die unteren 10\% des Leistungsspektrums zu prüfen. Insgesamt konnte sich der frühe Wortschatz mit 25 Monaten als guter alleiniger Prädiktor späterer Leistungen (mit 28 Monaten) bewähren. Für den Bereich Wortschatz ergab 
die Hinzunahme der MaxLU als Prädiktorvariable zum frühen Wortschatz eine Erhöhung der korrekt identifizierten „Lowscorer“ von 69\% auf 78\%. Für den Bereich Grammatik, konnte die Hinzunahme der MaxLU zum frühen Wortschatz keine signifikante Erhöhung der Trefferquote für die „Lowscorer“ erbringen.

Überwiegend wird in den Studien der Zusammenhang früher Sprachdaten (unter anderem auch MLU) mit späteren Sprachleistungen untersucht, wobei unterschiedliche Diagnosemethoden zur Anwendung kommen und die Ergebnisse auch bezogen auf die Erhebungsmethoden bewertet werden.

Die MLU im frühen Kindesalter zeigt signifikante Korrelationen $\mathrm{zu}$ späterem Sprachoutcome. So fanden Walker et al. (1994) bei einer Stichprobe von 32 Kindern ähnlich hohe und zum Teil sogar höhere Korrelationen der MLU (gemessen im Alter von 7 - 36 Monaten) mit späteren rezeptiven und expressiven Sprachleistungen im Kindergarten- und Grundschulalter im Vergleich zu den frühen Wortschatzdaten. Dieses lässt vermuten, dass die Hinzunahme der MLU (zu den frühen Wortschatzdaten) in die Vorhersageberechnungen für späteres Sprachoutcome die Varianzaufklärung (insbesondere für die späteren Grammatikmaße) signifikant verbessern könnte. Auf der anderen Seite zeigten sich in den Untersuchungen von Bornstein und Haynes (1998) an einer deutlich größeren Stichprobe (n = 154) niedrigere Werte für die Korrelationen der MLU (im Alter von 20 Monaten) mit späteren Ergebnissen (im Alter von 48 Monaten) im Verbalteil des WPPSI (Wechsler, 1989) von .15 als für die Korrelationen der frühen Wortschatzdaten mit späteren WPPSI Daten $(r=.27)$. Auch Feldman et al. (2005) berichten von Korrelationen der MLU im Alter von 2 Jahren mit späteren Sprachdaten im Alter von 3 Jahren gemessen an einer Stichprobe von 113 Kindern in der Höhe von .47 - .53, welche niedriger als die Korrelationen der frühen Wortschatzdaten zu späterem Sprachoutcome sind. Hier beliefen sich die Korrelationen auf .52 - .70.

Aufgrund der größeren Stichproben in letztgenannten Untersuchungen ist von einer zuverlässigeren Schätzung der wahren Korrelationswerte auszugehen. Dieses führt zu der Annahme, dass frühe Wortschatzdaten tendenziell höher mit späterem Sprachoutcome korrelieren als frühe MLU Werte und somit keine zusätzliche signifikante Varianzaufklärung durch die MLU Daten für die Vorhersage späterer Sprachleistungen zu erwarten ist. Untermauert wird diese Annahme durch Aussagen aus aktuelleren Untersuchungen zur Stabilität früher Sprachentwicklungsdaten. So fanden Reese und Read (2000) im Alter von $1 ; 7$ bis $2 ; 1$ Jahren geringere Stabilitätswerte für die MLU $(r=.57)$ als für den expressiven Wortschatz $(r=.81)$. Auch andere Untersuchungen weisen für die MLU insbesondere eine geringere heterotype Stabilität aus als für den frühen Wortschatz (Bornstein et al., 2004). Bei 
geringerer Stabilität ist in logischer Konsequenz ebenso von geringeren Varianzaufklärungsbeiträgen für spätere Sprachleistungen auszugehen.

Als Fazit aus dem theoretischen Hintergrund lässt sich die folgende psychologische Hypothese ableiten:

PH: Frühe grammatische Maße können die Vorhersage späterer Sprachleistungen im Vorschul- und Grundschulalter aus dem frühen Wortschatz nicht substanziell verbessern.

\subsection{Ergebnisse}

Zur Vorhersage der Sprachleistungen zu t $t_{1}\left(4 ; 0\right.$ Jahre), $t_{2}$ (5;0 Jahre) und $t_{3}$ (7;10 Jahre) wurde für alle Regressionsanalysen der Prozentrang im ELAN (expressiver Wortschatzumfang gemessen mittels Parent Report $\mathrm{zu} \quad \mathrm{t}_{0}$ ) als Prädiktorvariable verwendet. Als Kriteriumsvariablen für die Vorhersage späterer Wortschatzleistungen fungierten $\mathrm{zu}_{1}$ und $\mathrm{t}_{2}$ die Rohwerte im AWST 3-6 und $\mathrm{zu} \mathrm{t}_{3}$ die Rohwerte im Untertest „Wortschatz“ aus dem HAWIK III. Für die rezeptiven grammatischen Fähigkeiten wurden die Rohwerte im Untertest „Verstehen von Sätzen“ aus dem SETK 3-5 zu t $t_{1}$ und $t_{2}$ sowie die Rohwerte im Untertest „Verstehen grammatischer Strukturformen“ aus dem HSET $\mathrm{zu}_{3}$ in die Regressionsanalysen eingegeben. Als Kriteriumsvariablen für die Berechnung der Vorhersage expressiver grammatischer Leistungen fungierten die Rohwerte aus dem Untertest „Morphologische Regelbildung“ (SETK 3-5) zu $\mathrm{t}_{1}$ und $\mathrm{t}_{2}$ sowie die Rohwerte aus dem Untertest „Plural-Singular-Bildung“ (HSET) zu $\mathrm{t}_{3}$. Für die Vorhersage der Satzgedächtnisleistungen gingen als Kriteriumsvariablen die Rohwerte aus dem Untertest „Satzgedächtnis“ (SETK 3-5) zu $\mathrm{t}_{1}$ und $\mathrm{t}_{2}$ sowie die Rohwerte aus dem Untertest „Imitation grammatischer Strukturformen“ (HSET) zu t 3 in die Berechnungen ein.

Der Prozentrang im ELAN wurde als Prädiktorvariable im ersten Block mit Einschlussverfahren und die MLU als Prädiktorvariable im zweiten Block mit schrittweisem Verfahren in die hierarchische Regressionsanalyse eingegeben, um sicherzustellen, dass zunächst immer die Varianzaufklärungen aus dem Prozentrang im ELAN berechnet wurden und die MLU nur hinzugenommen wurde, wenn sie darüber hinaus noch zusätzlich zu einem signifikanten Varianzaufklärungsbeitrag führte. Das statistische Datenverarbeitungsprogramm SPSS schloss bei allen Berechnungen die zweite Prädiktorvariable aus nicht nachvollziehbaren Gründen aus. Dieser Ausschluss der MLU erfolgte bei Herausnahme eines Kindes aus dem bestehenden Datensatz nicht mehr. Die Analyse der Rohdaten dieses einen Kindes (wie auch der Gesamtstichprobe) zeigte jedoch keine systematischen Auffälligkeiten, 
welche diesen Tatbestand ausreichend erklärt und den kompletten Ausschluss der Daten des einen Kindes aus allen Berechnungen gerechtfertigt hätten. Das Vorliegen eines Kollinearitätsproblems konnte ebenfalls ausgeschlossen werden, da der Konditionsindex immer deutlich kleiner als 15 war. Aus diesem Grund wurde Forced Entry als Eingabeverfahren für die Regressionsanalysen gewählt. Dieses bedeutet, dass zunächst alle Regressionsanalysen wie in Kapitel 4 mit dem Prozentrang im ELAN als alleiniger Prädiktorvariable durchgeführt wurden und die nicht aufgeklärten Varianzanteile (standardisierte Residuen) aus diesen Regressionsanalysen gespeichert und als neue Kriteriumsvariablen für die folgenden Regressionsanalysen festgelegt wurden. Folgend wurden dann alle Regressionsanalysen mit der MLU als alleiniger Prädiktorvariable und den standardisierten Residuen aus den ersten Regressionsanalysen als Kriteriumsvariablen durchgeführt.

Augrund der Fülle an empirischen Vorhersagen werden exemplarisch nur die empirischen Vorhersagen für die Prädiktion späterer Wortschatzleistungen aufgestellt.

Empirische Vorhersagen:

EV 1: Die MLU gemessen mit dem ELAN führt $\mathrm{zu}$ keiner signifikanten Varianzaufklärung für die nicht aufgeklärten Varianzanteile aus der Regressionsanalyse mit der Prädiktorvariable Prozentrang im ELAN und der Kriteriumsvariable Rohwert im AWST 3-6 zu t.

EV 2: Die MLU gemessen mit dem ELAN führt $\mathrm{zu}$ keiner signifikanten Varianzaufklärung für die nicht aufgeklärten Varianzanteile aus der Regressionsanalyse mit der Prädiktorvariable Prozentrang im ELAN und der Kriteriumsvariable Rohwert im AWST 3-6 zu t2.

EV 3: Die MLU gemessen mit dem ELAN führt $\mathrm{zu}$ keiner signifikanten Varianzaufklärung für die nicht aufgeklärten Varianzanteile aus der Regressionsanalyse mit der Prädiktorvariable Prozentrang im ELAN und der Kriteriumsvariable Rohwert im Untertest „Wortschatz“ aus dem HAWIK III zu t3.

Tabelle 11 zeigt zum besseren Verständnis der weiteren Datenauswertung und Diskussion der Ergebnisse die Korrelationen von MLU und Prozentrang im ELAN mit allen späteren Sprachmaßen von $t_{1}$ bis $t_{3}$. 
Tabelle 11

Zusammenhänge früher grammatischer Fähigkeiten und früher Wortschatzleistungen (zu $\left.t_{0}\right)$ mit späteren Sprachmaßen von $t_{1}$ bis $t_{3}$

\begin{tabular}{|lll|}
\hline & MLU & Prozentrang im ELAN \\
\hline $\mathbf{t}_{\mathbf{1}}$ & $\boldsymbol{r}(\boldsymbol{p})$ & $\boldsymbol{r}(\boldsymbol{p})$ \\
WS & $.352(.018)^{*}$ & $.382(.009)^{* *}$ \\
VS 3-jährige & $.281(.092)$ & $.209(.152)$ \\
MR 3-jährige & $.046(.416)$ & $.090(.331)$ \\
VS 4-jährige & $.631(.000)^{* *}$ & $.463(.004)^{* *}$ \\
MR 4-jährige & $.566(.001)^{* *}$ & $.658(.000)^{* *}$ \\
SG & $.504(.003)^{* *}$ & $.424(.009)^{* *}$ \\
\hline $\mathbf{t}_{\mathbf{2}}$ & $\boldsymbol{r}(\boldsymbol{p})$ & $\boldsymbol{r}(\boldsymbol{p})$ \\
WS & $.435(.001)^{* *}$ & $.515(.000)^{* *}$ \\
VS & $.339(.008)^{* *}$ & $.364(.003)^{* *}$ \\
MR & $.276(.025)^{*}$ & $.376(.003)^{* *}$ \\
SG & $.247(.057)$ & $.432(.002)^{* *}$ \\
\hline $\mathbf{t}_{\mathbf{3}}$ & $\boldsymbol{r}(\boldsymbol{p})$ & $\boldsymbol{r}(\boldsymbol{p})$ \\
WS & $.331(.010)^{*}$ & $.276(.024)^{*}$ \\
HSET VS & $.158(.137)$ & $.215(.061)$ \\
PS & $.168(.122)$ & $.300(.015)^{*}$ \\
IS & $.253(.038)^{*}$ & $.384(.002)^{* *}$ \\
\hline
\end{tabular}

Anmerkungen. $\mathrm{t}_{1,2,3}=1$., 2. und 3. Messzeitpunkt, $\mathrm{WS}=$ Wortschatz, $\mathrm{VS}=$ rezeptive grammatische Fähigkeiten gemessen mit dem Untertest VS, MR expressive grammatische Fähigkeiten gemessen mit dem Untertest MR, SG = Satzgedächtnis, HSET VS = rezeptive grammatische Fähigkeiten gemessen mit dem Untertest HSET VS, PS = expressive grammatische Fähigkeiten gemessen mit dem Untertest PS, IS = Satzgedächtnis gemessen mit dem Untertest IS, $*=p<.05, * *=p<.01, r=$ Pearson Produkt Moment Korrelationen, $p=$ Fehlerwahrscheinlichkeit

Tendenziell höhere Korrelationen mit späterem Sprachoutcome bestehen für die MLU (im Vergleich zum Prozentrang im ELAN) lediglich bezogen auf drei Variablen: Untertest „Verstehen von Sätzen“ (SETK3-5) für die Stichprobe der vierjährigen Kinder zu t $\mathrm{t}_{1}$, Untertest „Satzgedächtnis“ zu t $t_{1}$ (SETK3-5; vorgesehen nur für die vierjährigen Kinder zu $\mathrm{t}_{1}$ ) sowie 
Untertest „Wortschatz“ aus dem HAWIK III zu t $t_{3}$. Die MLU und der Prozentrang im ELAN korrelierten in Höhe von $.76(p=.00)$.

Ausschließlich für den Untertest „Verstehen von Sätzen“ bei der Stichprobe der vierjährigen Kinder $\mathrm{zu} \mathrm{t}_{1}$ konnte die MLU einen signifikanten Varianzaufklärungsbeitrag bzgl. der Residuen aus der ersten Regressionsanalyse mit der Prädiktorvariable Prozentrang im ELAN leisten $\left(R_{\text {korr }}^{2}=.12 ; F=4.73 ; p=.04\right)$.

Somit gilt die empirische Vorhersage hinsichtlich des Untertests „Verstehen von Sätzen“ für die vierjährigen Kinder $\mathrm{zu} \mathrm{t}_{1}$ als nicht erfüllt. Alle anderen empirischen Vorhersagen haben sich als zutreffend bestätigt.

\subsection{Diskussion}

Die psychologische Hypothese („Frühe grammatische Maße können die Vorhersage späterer Sprachleistungen im Vorschul- und Grundschulalter aus dem frühen Wortschatz nicht substanziell verbessern.") gilt bei strenger Prüfung mit konjunktiver Verknüpfung aller empirischen Erwartungen als nicht bestätigt, denn eine der 14 empirischen Vorhersagen hat sich nicht erfüllt. Es gilt jedoch diese Aussage zu relativieren, denn lediglich für eine Teilstichprobe 31 Kindern ließen sich wider Erwarten zu einem Messzeitpunkt bezogen auf die rezeptiven grammatischen Leistungen substanzielle zusätzliche Varianzaufklärungen für die MLU berechnen. Die gleiche Teilstichprobe führt für die Berechnungen zur Variable Sprechbeginn (vgl. Kap. 6) zum einzigen signifikanten Ergebnis, das nicht konform mit der psychologischen Hypothese ist. Eine Stichprobenverzerrung könnte hierfür ursächlich sein.

Überwiegend zeigen die Ergebnisse auf Grundlage vorliegender Datenbasis keine zusätzlichen substanziellen Varianzaufklärungsbeiträge der frühen grammatischen Fähigkeiten für späteres Sprachoutcome über den frühen Wortschatz hinaus.

Bornstein \& Haynes (1998) sowie Feldman et al. (2005) berichten von geringeren Korrelationen der MLU mit späterem Sprachoutcome im Vergleich zum frühen Wortschatz, was für die Mehrheit aller Korrelationen auch in dieser Studie gilt. Für die MLU konnten Korrelationen mit späteren Sprachleistungen in Höhe von .25 - .63 und für den frühen Wortschatz Korrelationen von .28 - .66 errechnet werden. Es besteht also kein ausgeprägter Unterschied in der Höhe der Korrelationen.

Dieses unterstreicht neben der hohen Korrelation von MLU und frühem Wortschatz zu $\mathrm{t}_{0}$ $(r=.76)$ den gut belegten Befund, dass Wortschatz und Grammatik insbesondere im frühen Kindesalter in sehr engem Zusammenhang stehen. McGregor, Sheng \& Smith (2005) gehen davon aus, dass Grammatikentwicklung in höherem Zusammenhang mit 
Wortschatzentwicklung steht als mit dem chronologischen Alter. So ist es auch nicht verwunderlich, dass die frühen grammatischen Leistungen im Wesentlichen die gleichen Informationen repräsentieren, die im expressiven Wortschatzumfang enthalten sind.

Zur Erhebung der MLU mittels ELAN ist positiv anzumerken, dass die Eltern bei der Angabe von drei typischen Äußerungen ihres Kindes auf die ganze Bandbreite an sprachlichen Äußerungen des Kindes zurückgreifen können. Bei einer Erhebung der MLU über Spontansprachanalysen sowohl mit einem fremden Gesprächspartner als auch mit den Eltern, wird immer nur ein kleiner Ausschnitt der sprachlichen Äußerungsmöglichkeiten des Kindes betrachtet. Entscheidend für die Reliabilität und Validität der Messung der MLU ist zum einen die Anzahl an Fragen, mit denen ein Kind zu Sprachäußerungen angeregt wird und zum anderen die Anzahl der dokumentierten Äußerungen aus der die MLU errechnet wird. Johnston, Miller, Curtiss und Tallal (1993) konnten beobachten, dass die MLU abnahm, je mehr Fragen dem Kind in der Untersuchungssituation gestellt wurden. Für die Erhebung der MLU im ELAN wurde das Kind nicht durch Fragen (in unnatürlichem Setting) zu Sprachäußerungen angeregt, was für die Güte der Messung spricht. Fraglich ist allerdings wie gut sich die Eltern im free recall, also freiem Erinnern (im Gegensatz zu recognition, also Wiedererkennung vorgegebener Beispiele) an drei typische Äußerungen ihres Kindes erinnern können und wie sehr ihre Angaben geprägt sind von der Erinnerung an besonders markante (weil lustige oder nervige) Äußerungen.

Gavin und Giles (1996) betonen, dass die Güte der Messung für die MLU abhängt von der Anzahl an Sprachäußerungen, die zur Berechnung zur Verfügung stehen und verweisen auf ein Minimum von 175 Sprachäußerungen, um Messungen von akzeptabler Reliabilität zu erhalten. Geht man davon aus, dass die Eltern für die Angaben im ELAN auf eine weitaus größere Menge an Äußerungen ihres Kindes zurückgreifen können (mit den entsprechenden gedächtnispsychologischen Bedenken), dann ist trotz der Auswahl von drei typischen Äußerungen von einer zuverlässigen Messung der MLU mittels ELAN auszugehen. Die hohen Korrelationen zum frühen Wortschatz sowie die überwiegend signifikanten Korrelationen (mittlerer bis hoher Effektstärke) zu späterem Sprachoutcome bestätigen dieses.

Die Operationalisierung früher grammatischer Fähigkeiten über andere Maße als die MLU ist aufgrund der vielfältigen Forschungsergebnisse, welche die MLU als reliablen und validen Indikator für den grammatischen Leistungsstand ausweisen und hohe Zusammenhänge zu anderen Indikatoren der Grammatikentwicklung anzeigen, nicht anzuraten. Auch die sehr hohe Übereinstimmung der Ergebnisse von MLU gemessen in Morphemen und MLU gemessen in Worten (Rice et al., 2006) unterstützt die in dieser Studie gewählte 
Operationalisierung des Konstruktes „grammatische Fähigkeiten“ im frühen Kindesalter mittels der MLU in Worten.

Die Aussagen von Rice et al. (2006) zum Bestehen robuster Reliabilität und Validität bezogen auf das frühe Sprachmaß MLU sowohl für den gestörten als auch den normalen Spracherwerb im längsschnittlichen Design, legt die Annahme nahe, dass die hier für den normalen Spracherwerb berichteten Ergebnisse gleichermaßen für den gestörten Spracherwerb gelten.

Einschränkend anzumerken ist, dass die in dieser Studie dargestellten Ergebnisse nur für die zugrunde liegende Datenbasis in ihrer spezifischen Zusammensetzung bezogen auf Wortschatzgröße und Lebensalter der Kinder zu t $\mathrm{t}_{0}$ gelten.

Für die Repräsentativität der an dieser Stichprobe erhobenen Ergebnisse zur wenig beforschten psychologischen Hypothese (,Frühe grammatische Maße können die Vorhersage späterer Sprachleistungen im Vorschul- und Grundschulalter aus dem frühen Wortschatz nicht substanziell verbessern.“) sprechen die mit dem Forschungsstand gut vergleichbaren Ergebnisse zu den vielfältig untersuchten psychologischen Hypothesen in Kapitel 3 und 4.

Im Fazit konnte die MLU die Vorhersage späterer Sprachleistungen aus dem frühen expressiven Wortschatz nicht bedeutsam verbessern und der Forderung von Feldman et al. (2005) nach der Suche anderer Maße außer MLU und Wortschatzumfang zur Vorhersage späterer Sprachleistungen kann zugestimmt werden.

In Kapitel 6 wird als weitere Variable neben Wortschatz und MLU die Variable „Sprechbeginn“ auf das Bestehen bedeutsamer Zusammenhänge zu späterem Sprachoutcome untersucht. Da frühe grammatische Fähigkeiten keine Verbesserung der Vorhersage späterer Sprachleistungen aus dem frühen Wortschatz erbrachten, wird das Augenmerk auf den frühen Wortschatz selbst gelegt. Dieser war lediglich quantitativ betrachtet und als Variable „expressiver Wortschatzumfang“ in die Regressionsanalysen eingefügt worden (vgl. Kap. 4). Qualitative Aspekte des frühen Wortschatzes könnten jedoch zur Verbesserung der Varianzaufklärung beitragen und werden in Kapitel 7 diesbezüglich untersucht. 


\section{Sprechbeginn - Zusammenhänge zu späteren Sprachleistungen}

\subsection{Theoretischer Hintergrund}

Um den ersten Geburtstag herum beginnen Kinder mit etwas, was kein anderes Lebewesen macht: sie sprechen (vgl. Bates, Bretherton \& Snyder, 1988; Francescato, 1973; Nelson, 1973; Stern \& Stern, 1922). Fenson et al. (1994) betonen die große interindividuelle Variabilität im Auftreten des ersten Wortes und andere Autoren benennen Zeitpunkte zwischen 8 und 18 Monaten für die Äußerung erster Worte (Bloom, Tinker \& Margulis, 1993; Bühler, 1928; McCarthy, 1966). Bloom et al. (1993) berichten von einem durchschnittlichen Alter von 13.26 Monaten für den Sprechbeginn und Kauschke (2003) definiert erste echte Worte als solche, die situationsunabhängig sind und über einen festen inhaltlichen Bezug verfügen. Eltern warten gespannt auf diesen wichtigen Meilenstein der kindlichen Entwicklung und reagieren mit großer Sorge bei Verzögerung in der Produktion erster Wörter. Snyder, Bates und Bretherton (1981) drücken dieses wie folgt aus: „For most parents the infant`s first word marks a clear and important transition that changes the child`s status as a social partner" (S. 565). In der Regel werden die ersten Worte produziert, wenn der rezeptive Wortschatz zwischen 20 und 30 Wörtern (Nelson, 1988) bzw. zwischen 11 und 50 Wörtern (Bates, 1993) umfasst. Doch einige Kinder sind bereits in der Lage 200 Wörter zu verstehen, bevor sie ihr erstes Wort sprechen, und wenige Kinder zeigen in ihrer Wortschatzentwicklung kaum eine Differenz zwischen Verstehen und Produktion.

Es stellt sich die Frage, wann man vom ,ersten Wort“ eines Kindes sprechen kann. Für die Unterscheidung, ob aktive Sprache noch Lallwörter oder bereits Worte im eigentlichen Sinn sind, lässt sich eine Definition von Lewis (1954, zitiert nach Francescato, 1973) heranziehen:

When we say that a child has begun to use words in a meaningful way, we are describing this behaviour: that now in a specific situation he consistently utters a specific sound group whose phonetic patterns is either borrowed from the adult language or influenced by its forms. (S. 77)

Snyder et al. (1981) sprechen vom „ersten Wort“, wenn ein Kind die Idee der Namensgebung verstanden hat und Kamhi (1986) bezeichnet dieses als „naming insight“.

Es ist wichtig zu betonen, dass erste Wörter weder einfache Imitationen noch plötzliche, zufällige Phänomene sind. Vielmehr bedarf es bestimmter Vorläuferfertigkeiten wie Wahrnehmungsleistungen, sprachrelevanter sozialer Fertigkeiten und Kognitionsleistungen sowie Gedächtnis- und phonologischer Fähigkeiten, damit Wörter entstehen können. Zieht man dieses in Betracht, wird verständlich, dass der Sprechbeginn als Ausdruck für den Entwicklungsstand sprachrelevanter Vorläuferfertigkeiten Rückschlüsse auf die allgemeinen 
Sprach- und Sprechleistungen eines Kindes zulassen könnte. Hat das Alter, in dem erste Worte gesprochen werden, einen bedeutsamen Zusammenhang zu späteren Sprachleistungen oder erlaubt es gar substanzielle Vorhersagen späterer Sprachleistungen? Zu dieser Frage sind kaum Studien zu finden. Kiese-Himmel (1999) berichtet von einem signifikanten Varianzaufklärungsbeitrag des Sprechbeginns erster Worte (als einer von sechs Prädiktorvariablen) für den späteren produktiven Wortschatz bei hörgestörten Kindern.

Wenn auch überzeugend ist, dass die ersten Worte ein Indikator für bestimmte Vorläuferfertigkeiten der Sprachentwicklung darstellen, so bleibt fraglich, wie entscheidend der Zeitpunkt ist, zu dem erste Worte geäußert werden. Zum einen ist dieser Zeitpunkt hochvariabel und lässt keinen Zusammenhang zum rezeptiven Wortschatz erkennen. So wird berichtet, dass Kinder über einen ganz unterschiedlich großen rezeptiven Wortschatz verfügen, bevor sie erste Worte sprechen (siehe Bates, 1993). Zum anderen muss man sich fragen, ob Eltern überhaupt in der Lage sind, zuverlässig zu beurteilen, wann ihr Kind Wörter im eigentlichen Sinne spricht und somit über „naming insight“ verfügt bzw. wann die aktiven Äußerungen des Kindes noch als Lallwörter zu bewerten sind. Darüber hinaus bleibt zu bedenken, welchen Informationsgehalt das frühe Sprechalter z.B. im Vergleich zum frühen expressiven Wortschatzumfang hat, dessen prädiktive Validität für spätere Sprachleistungen gut belegt ist. Fakt bleibt, dass im Alltag vieler Familien sowie im klinischen Alltag (z.B. in den pädiatrischen Praxen) der Sprechbeginn einen hohen Stellenwert in der Entwicklungsdiagnostik einnimmt und Eltern mit großer Sorge bei verspäteten ersten Worten ihres Kindes reagieren. Aus diesem Grunde soll das von den Eltern im ELAN dokumentierte Alter der Äußerung erster Worte untersucht werden auf Zusammenhänge zu späterem Sprachoutcome.

Aus dem theoretischen Hintergrund wird folgende psychologische Hypothese abgeleitet:

PH: Früher Sprechbeginn steht in keinem bedeutsamen Zusammenhang zu (relativ) besseren Sprachleistungen in der frühen Kindheit bis ins Grundschulalter.

\subsection{Ergebnisse}

Als Sprachmaße wurden im mittleren Alter von 22 Monaten $\left(t_{0}\right)$, 4;0 Jahren $\left(t_{1}\right)$, 5;0 Jahren $\left(\mathrm{t}_{2}\right)$ und 7;10 Jahren $\left(\mathrm{t}_{3}\right)$ die Leistungen in den Bereichen Wortschatz, rezeptive und expressive grammatische Fähigkeiten und Satzgedächtnis erhoben und mit dem Alter erster Worte (Sprechbeginn) in Zusammenhang gesetzt. Hierbei gehen in die Korrelationsanalysen für den Wortschatz zu to der Prozentrang für den expressiven Wortschatzumfang im ELAN, $\mathrm{zu} \mathrm{t}_{1}$ und $\mathrm{t}_{2}$ die Rohwerte im AWST 3-6 und $\mathrm{zu} \mathrm{t}_{3}$ die Rohwerte aus dem Untertest 
„Wortschatz“ (HAWIK III) ein. Für die rezeptiven grammatischen Fähigkeiten werden zu $\mathrm{t}_{1}$ und $t_{2}$ die Rohwerte des Untertests „Verstehen von Sätzen“ (SETK3-5) und $\mathrm{zu} \mathrm{t}_{3}$ die Rohwerte im Untertest „Verstehen grammatischer Strukturformen“ (HSET) mit dem Alter erster Worte (ELAN) korreliert. Bezogen auf die expressiven Leistungen fungieren die Rohwerte im Untertest „Morphologische Regelbildung“ (SETK 3-5) zu $\mathrm{t}_{1}$ und $\mathrm{t}_{2}$ sowie die Rohwerte aus dem Untertest „Plural-Singular-Bildung“ (HSET) zu $t_{3}$ als Variablen in den Korrelationsanalysen. Zusätzlich ging als frühes Maß für die expressiven grammatischen Fähigkeiten $\mathrm{zu} \mathrm{t}_{0}$ die MLU in Worten (gemessen mit dem ELAN) in die Berechnungen ein. Für das Satzgedächtnis werden die Rohwerte im Untertest „Satzgedächtnis“ (SETK 3-5) zu t und $t_{2}$ sowie die Rohwerte im Untertest „Imitation grammatischer Strukturformen“ (HSET) mit dem Sprechbeginn im ELAN korreliert. Der „Sprechbeginn“ wurde über das von den Eltern im ELAN dokumentierte Alter erster Worte in Monaten operationalisiert. Pearson Produkt-Moment-Korrelationen (zweiseitiges Signifikanzniveau) mit den Sprachmaßen zu $t_{0}$ (expressiver Wortschatzumfang und MLU), den Sprachmaßen zu $t_{1}$ und $t_{2}$ (AWST 3-6, VS, MR und SG) sowie den Sprachmaßen zu t 3 (HAWIK WS, HSET VS, PS und IS) wurden berechnet.

Bei der großen Anzahl an empirischen Vorhersagen, werden exemplarisch nur die empirischen Vorhersagen für den Erhebungszeitpunkt $t_{0}$ formuliert.

Empirische Vorhersagen:

EV 1: Es werden keine signifikanten Korrelationen zwischen dem Sprechbeginn im ELAN und dem Prozentrang für den expressiven Wortschatzumfang im ELAN erwartet.

EV 2: Es werden keine signifikanten Korrelationen zwischen der MLU in Worten gemessen mit dem ELAN und dem Sprechbeginn im ELAN erwartet.

Tabelle 12 veranschaulicht die Ergebnisse der Korrelationsanalysen für alle Sprachoutcomevariablen von $\mathrm{t}_{0}$ bis $\mathrm{t}_{3}$. Zur besseren Einordnung der Ergebnisse sind im Vergleich die entsprechenden Korrelationen der jeweiligen Sprachoutcomevariablen mit dem Prozentrang im ELAN angegeben. 
Tabelle 12

Zusammenhänge zwischen Sprechbeginn, frühem Wortschatz und den Sprachmaßen von früher Kindheit bis ins Grundschulalter ( $t_{0}$ bis $t_{3}$ )

\begin{tabular}{|lll|}
\hline & Sprechbeginn & Prozentrang im ELAN \\
\hline $\mathbf{t}_{\mathbf{0}}$ & $\boldsymbol{r}(\boldsymbol{p})$ & $\boldsymbol{r}(\boldsymbol{p})$ \\
MLU & $-.028(.849)$ & $.763(.000)^{* *}$ \\
PR ELAN & $-.067(.632)$ & - \\
\hline $\mathbf{t}_{\mathbf{1}}$ & $\boldsymbol{r}(\boldsymbol{p})$ & $\boldsymbol{r}(\boldsymbol{p})$ \\
WS & $.181(.297)$ & $.382(.009)^{* *}$ \\
VS 3-jährige & $.195(.340)$ & $.209(.152)$ \\
MR 3-jährige & $.146(.477)$ & $.090(.331)$ \\
VS 4-jährige & $-.261(.188)$ & $.463(.004)^{* *}$ \\
MR 4-jährige & $-.404(.037)^{*}$ & $.658(.000)^{* *}$ \\
SG & $.062(.759)$ & $.424(.009)^{* *}$ \\
\hline $\mathbf{t}_{\mathbf{2}}$ & $\boldsymbol{r}(\boldsymbol{p})$ & $\boldsymbol{r}(\boldsymbol{p})$ \\
WS & $-.023(.873)$ & $.515(.000)^{* *}$ \\
VS & $.055(.703)$ & $.364(.003)^{* *}$ \\
MR & $.057(.695)$ & $.376(.003)^{* *}$ \\
SG &. $.149(.346)$ & $.432(.002)^{* *}$ \\
\hline $\mathbf{t}_{\mathbf{3}}$ & $\boldsymbol{r}(\boldsymbol{p})$ & $\boldsymbol{r}(\boldsymbol{p})$ \\
WS & $.115(.438)$ & $.276(.024)^{*}$ \\
HSET VS & $.155(.287)$ & $.215(.061)$ \\
PS & $.041(.782)$ & $.300(.015)^{*}$ \\
IS & $.039(.791)$ & \\
\hline
\end{tabular}

Anmerkungen. $\mathrm{t}_{0}=$ Messzeitpunkt 2001, $\mathrm{t}_{1,2,3}=1$., 2. und 3. Messzeitpunkt, $\mathrm{MLU}=$ Mean length of Utterances, PR ELAN $=$ Prozentrang im ELAN, WS $=$ Wortschatz, VS $=$ rezeptive grammatische Fähigkeiten gemessen mit dem Untertest VS, MR expressive grammatische Fähigkeiten gemessen mit dem Untertest MR, SG = Satzgedächtnis, HSET VS = rezeptive grammatische Fähigkeiten gemessen mit dem HSET VS, PS = expressive grammatische Fähigkeiten gemessen mit dem Untertest PS, IS = Satzgedächtnis gemessen mit dem Untertest IS, $*=p<.05,{ }^{*}=p<.01, r=$ Pearson Produkt Moment Korrelationen, $p=$ Fehlerwahrscheinlichkeit 
Für den Sprechbeginn errechneten sich sowohl positive als auch negative Korrelationen mit den Sprachoutcomevariablen von $t_{0}$ bis $t_{3}$. Lediglich für die Stichprobe der vierjährigen Kinder $\mathrm{zu} \mathrm{t}_{1} \mathrm{im}$ Untertest „Morphologische Regelbildung“ ließ sich eine signifikante Korrelation mit dem Sprechbeginn in Höhe von -.404 $(\mathrm{p}=.037)$ berechnen. Die entsprechende Korrelation für den Prozentrang im ELAN mit dem Untertest „Morphologische Regelbildung“ $\mathrm{zu} \mathrm{t}_{1}$ (4-jährige Kinder) belief sich auf .658 $(p=.000)$. Zwischen frühem expressiven Wortschatzumfang und Sprechbeginn bestand keine signifikante Korrelation ( $r=$ $-.067 ; p=.632)$.

Alle empirischen Vorhersagen mit Ausnahme der empirischen Vorhersage für den Zusammenhang von Sprechbeginn und „Morphologischer Regelbildung“ $\mathrm{zu} \mathrm{t}_{1}$ für die vierjährigen Kinder haben sich erfüllt.

\subsection{Diskussion}

Die psychologische Hypothese (Früher Sprechbeginn steht in keinem bedeutsamen Zusammenhang $\mathrm{zu}$ [relativ] besseren Sprachleistungen in der frühen Kindheit bis ins Grundschulalter) muss bei strenger Prüfung mit fakultativer Verknüpfung aller empirischen Vorhersagen aufgrund der Ergebnisse abgelehnt werden, da sich eine der empirischen Vorhersagen nicht erfüllt hat.

Dieses sollte aufgrund der mehrheitlich bestätigten empirischen Vorhersagen (15 von 16) nicht überbewertet werden. Denn lediglich für eine Teilstichprobe von 31 Kindern zu einem Messzeitpunkt $\left(\mathrm{t}_{1}\right)$ konnten signifikante Zusammenhänge für den Sprechbeginn berechnet werden. Bei der geringen Stichprobengröße von $n=31$ Kindern und in Anbetracht des mehrheitlichen Nichtbestehens von substanziellen Zusammenhängen könnte dieses Ergebnis durch Stichprobenverzerrungen bedingt sein, denn für die gleiche Teilstichprobe ergaben sich auch bezogen auf die MLU als zusätzlichem Prädiktor für spätere Sprachleistungen das einzige nicht erwartungskonforme Resultat (vgl. Kap. 5).

Wenn tatsächlich ein inhaltlich begründeter Zusammenhang zwischen Sprechbeginn und späteren Sprachleistungen bestünde, müssten für alle Sprachoutcomevariablen negative Korrelationen berechnet werden können. Denn: Je höher das Alter in Monaten für den Sprechbeginn, umso schlechtere Sprachleistungen $\mathrm{zu} \mathrm{t}_{0}$ bis $\mathrm{t}_{3}$ wären dann $\mathrm{zu}$ erwarten. Dass sich neben negativen auch positive Korrelationen für Sprechbeginn und Sprachoutcomevariablen berechnen ließen, relativiert ebenfalls das Bestehen einer einzigen signifikanten Korrelation. 
Gerade für die frühen Sprachmaße $\mathrm{zu}_{0}$ wären am ehesten Zusammenhänge zum Sprechbeginn aufgrund der zeitnahen Erhebung zu erwarten gewesen. Für die MLU sowie für den expressiven Wortschatzumfang $\mathrm{zu}_{\mathrm{t}}$ ergaben sich aber ebenso keine signifikanten Korrelationen mit dem Sprechbeginn. Im Unterschied zu den anderen Erhebungszeitpunkten lässt sich zu to auf die Gesamtstichprobe zur Entwicklung des ELANs von 186 Kindern zurückgreifen. Auch für diese große Stichprobe ergeben sich keine bedeutsamen Zusammenhänge zwischen expressivem Wortschatzumfang, MLU und Sprechbeginn ( $r=$ $-.019[p=0.81]$ und $r=-.02[p=.80])$. Sehr wohl aber besteht auch für die große Stichprobe eine signifikante Korrelation von .71 $(p=.00)$ zwischen MLU und expressivem Wortschatzumfang. Dieses spricht für die Repräsentativität der an vorliegender Datenbasis erzielten Ergebnisse und für die Nützlichkeit des Maßes früher expressiver Wortschatzumfang im Vergleich zum Sprechbeginn. Gleichermaßen für die Repräsentativität der Ergebnisse spricht die repräsentative Verteilung der Variable Sprechbeginn in dieser Untersuchungsstichprobe. Im Mittel sprechen die Kinder der hier untersuchten Stichprobe ihr erstes Wort mit 13.28 Monaten $(S D=3.0)$. Der früheste Sprechbeginn war mit 9 Monaten und der späteste Sprechbeginn mit 20 Monaten von den Eltern im ELAN angegeben worden. Sowohl der mittlere Sprechbeginn (um den ersten Geburtstag) als auch die hohe interindividuelle Variabilität stimmen gut mit Angaben aus der Forschungsliteratur überein. Bloom et al. (1993) berichten z.B. über einen mittleren Sprechbeginn von 13.26 Monaten.

Im klinischen Setting wird der Sprechbeginn meist mit Hinblick auf die Vorhersage späterer sprachlicher Leistungen verwendet. Die Ergebnisse dieser Dissertation zeigen hingegen, dass die Variable Sprechbeginn insbesondere im Vergleich zum frühen expressiven Wortschatzumfang denkbar ungeeignet für Vorhersagen späterer Wortschatz-, Grammatiksowie Satzgedächtnisleistungen im Alter von 22 Monate bis 7;10 Jahre ist. Geht es um die Vorhersage späterer Sprachmaße z.B. im Alter von 4;0 Jahren liefert der frühe expressive Wortschatzumfang gute Vorhersagen (vgl. Kap. 4), wobei der ELAN die Erhebung des frühen expressiven Wortschatzes schon im Alter von 16 Monaten ermöglicht. Für noch frühere Vorhersagen z.B. im Alter von 10 Monaten ist die Validität und Reliabilität der Ergebnisse fraglich (vgl. Kap. 4) und es müssten Vorläuferfertigkeiten bzw. rezeptive Sprachmaße und deren zuverlässige und gültige Erfassung weiter erforscht bzw. entwickelt werden.

Zusammenfassend scheint das Alter, in dem erste Worte gesprochen werden, keinen bedeutsamen Zusammenhang zu späteren Sprachleistungen zu haben, was einerseits durch die wenig zuverlässige Beurteilung der Eltern bzgl. des Vorliegens „echter Worte“ bedingt sein 
mag. Zum anderen scheint nicht entscheidend zu sein, wann ein Kind aus den bestehenden Ressourcen ein erstes Wort bildet, sondern wie viele solcher „Sprachbausteine“ es in der frühen Kindheit in der Lage ist aufzubauen und auszudrücken. Als Implikation für den klinischen Alltag ergeben sich ein vorsichtigerer Umgang mit den Ergebnissen der Standardfrage „Wann sprach ihr Kind erste Worte“ und ein Absehen von unfundierter Beunruhigung der Eltern, deren Kind vor 18 Monaten keine ersten Worte äußert. 


\section{Qualitative Analysen des frühen Wortschatzes}

In der aktuellen Forschung zu Spracherwerbsstörungen wird von einem Paradigmenwechsel in der Betrachtung semantisch-lexikalischer Störungen bei Kindern gesprochen. Denn die bisher übliche Diagnose eingeschränkter Wortschatz (ohne Spezifizierungen der Art der Einschränkungen) mit dem daraus abzuleitenden Behandlungsziel des Aufbaus eines altersgemäßen Wortschatzes konnte $\mathrm{zu}$ keinen befriedigenden therapeutischen Erfolgen führen (Vogt, 2003) und entspricht nicht mehr dem inzwischen wesentlich umfänglicheren Kenntnisstand zum frühen Spracherwerb. Vielmehr ist es die differenzierte Diagnostik des rezeptiven und expressiven Wortschatzes auch im Hinblick auf qualitative Aspekte wie z.B. der Komposition des Wortschatzes, welche Hinweise für eine erfolgreiche Therapieplanung liefern kann.

Auch der sprachpsychologischen Forschung zur normalen Sprachentwicklung ist die Aufforderung nach weitergehenden Analysen der frühen Sprachdaten zu entnehmen. So fordern Feldman et al. (2005) als Fazit ihrer Untersuchungen zur prädiktiven Validität früher Sprachmaße weitergehende Betrachtungen über die Verwendung der üblichen Variablen „expressiver Wortschatzumfang“ und „MLU“ hinaus und Berglund und Eriksson (2000) betonen, dass bewährte Parent Report Verfahren wie z.B. die CDI, die Untersuchung und Beschreibung von genereller Entwicklung und Variation verschiedener linguistischer Aspekte an großen Stichproben ermöglichen.

Eine Möglichkeit der differentielleren Betrachtung der frühen Sprachdaten zu $\mathrm{t}_{0}$ bietet die qualitative Analyse des expressiven Wortschatzes. Für diese qualitative Analyse lässt sich zum einen die Zusammensetzung des frühen expressiven Wortschatzes bezogen auf die Wortarten als Teilmengen des Gesamtwortschatzes analysieren. Darüber hinaus können Zusammensetzung und Diversifizität des frühen Lexikons über die Anzahl semantischer Kategorien sowie die Summe von Inhalts- und Funktionswörtern und deren Verhältnis zueinander operationalisiert werden.

\subsection{Wortarten}

\subsubsection{Theoretischer Hintergrund}

Grundsätzlich werden in der Literatur unterschiedliche Termini für die Beschreibung der Wortarten verwendet, die z. T. verwirrend sind. In Anlehnung an die aktuellste Dudenauflage - Deutsche Grammatik - kurz gefasst (Dudenreaktion, 2006) lassen sich neun Wortarten unterscheiden: Verben (konjugierbar), Substantive, Adjektive, Artikel und Pronomen (deklinierbar) sowie Adverbien, Präpositionen, Konjunktionen und Interjektionen (nicht 
flektierbar). Der Terminus „Nomen“, der von vielen Autoren synonym für „Substantive“ verwendet wird, ist sprachwissenschaftlich im weiteren Sinne definiert als ,alle deklinierbaren Wortarten mit Ausnahme der Artikel und Pronomen“ (Dudenreaktion, 2001). Ich ersetze diesen Terminus mit Ausnahme von wörtlicher Zitierung durch den eindeutigen Terminus „Substantive“.

Die Entwicklung des Lexikons ist durch eine Zunahme der Menge an Worten und der Elaboration auf der Bedeutungsseite charakterisiert. Darüber hinaus ist eine zunehmende Vielfalt der Wortarten insbesondere im Alter von 1;8 bis 2;6 Jahren charakteristisch für die Wortschatzentwicklung (Bassano, Eme \& Champaud, 2005). Aufgrund unterschiedlicher Erhebungs- und Analysemethoden und insbesondere in Abhängigkeit von der untersuchten Sprache (Deutsch, Englisch, Koreanisch, etc.) ergeben sich uneinheitliche Ergebnisse zur Wortartenentwicklung. Es lassen sich jedoch allgemeine Tendenzen feststellen. Nach frühem Auftreten von personal-sozialen Wörtern (wie Grüßen und Floskeln) sowie Eigennamen und Lautmalereien ist sprachübergreifend festzustellen, dass Substantive früh auftreten und sich auf konkrete Objekte beziehen. Des Weiteren sind relationale Wörter (wie z.B. „ab“, „mehr“, „da“, „weg“) wesentlich für die frühe Phase des Lexikonerwerbs.

Die Frage, welche Wortart den frühen Spracherwerb dominiert, wird divergent beantwortet. Kupfer (1959) berichtet, dass die Substantive den größten prozentualen Anteil am expressiven Wortschatz im 2. Lebensjahr einnehmen, Verben den zweitgrößten, Adjektive den drittgrößten. Gleichzeitig wird vom Autor angemerkt, dass der Substantivanteil mit fortschreitendem Alter abnehme, der Anteil an Verben und Adjektiven hingegen zunehme. Viele Autoren stimmen überein, dass Substantive bzw. Objektwörter im frühen expressiven Wortschatz (bis 50 Wörter) überwiegen (Bates, Marchman, Thal, Fenson, Dale, Reznick et al., 1994; Benedict, 1979; Snyder et al., 1981).

Die Dominanz der Substantive bzw. Objektwörter bzgl. der ersten 50 Wörter konnte auch in sprachvergleichenden Studien nachgewiesen werden (Au, Dapretto \& Song, 1994; Choi \& Gopnik, 1995; Kim, McGregor \& Thompson, 2000). Caselli, Bates, Casadio, Fenson, J., Fenson, L., Sanderl und Weir (1995) stellten in einer sprachvergleichenden Studie mit englischen und italienischen Kindern fest, dass bei allen Kindern die ersten 5 bis 10 Wörter Geräuschproduktionen, sog. „Routinen“ (z.B. soziale Ausdrücke wie „winke winke“ und tägliche Routinen wie „ham“ in Verbindung mit dem Essen), und Personennamen waren, also weder Substantive noch Verben. Bei der Analyse der ersten 50 bis 100 Wörter überwiegen jedoch nach Angaben der Autoren Objektwörter. 
Goldfield und Reznick (1990) verweisen auf den Einfluss von Schichtzugehörigkeit und Geschwisterposition auf das Verhältnis der Wortarten zueinander. Demnach verfügen erstgeborene Kinder aus der Mittelschicht über besonders viele Substantive und einen Wortschatzspurt nach den ersten 50 Wörtern. Zweit- und Drittgeborene aus sozial niedrigeren Schichten sind insgesamt langsamer in ihrer Entwicklung und das Verhältnis der Wortarten ist dabei ausgeglichener. So beläuft sich der Anteil an Objektnamen auf weniger als 50\%. Die Autoren postulieren einen Zusammenhang zwischen Substantivdominanz und Wortschatzspurt. Rescorla, Mirak und Singh (2000) sehen einen deutlichen Zusammenhang zwischen frühem Wortschatzwachstum und Substantivgebrauch, wenn der frühe Wortschatz mittels Vokalbechecklist erhoben wird. Die Kinder, die überwiegend Substantive erwerben, können ihren Wortschatzumfang schneller erhöhen als Kinder die andere Wortarten parallel erwerben. Hieraus schlussfolgern die Autoren, dass in der Behandlung von Late Talkern zunächst der Substantivwortschatz aufgebaut werden sollte, um ein schnelles Wortschatzwachstum gewährleisten zu können. Nach Bates et al. (1994) nimmt die Größe des Wortschatzes im Alter von 16 bis 30 Monaten konstant zu, dessen Zusammensetzung ändert sich jedoch substantiell. So besteht der expressive Wortschatz zunächst überwiegend aus Substantiven, für die in der frühen Sprachentwicklung ein steiler Anstieg verzeichnet werden kann (Maximum $=55 \%$ bei einem Wortschatzumfang von 101-200 Wörtern). Ab 100 Wörtern nimmt der Substantivanteil deutlich ab (bei einem Wortschatzumfang von 600 Wörtern $42 \%$ Substantive), der Anteil an Verben und Adjektiven hingegen stetig zu. Ab einem Gesamtwortschatz von 200 Wörtern (mit ca. 1;8 bis 2;0 Jahren) steigt der Anteil an Funktionswörtern (z.B. Artikel, Präpositionen, Hilfsverben) deutlich. Die Daten von Grimm et al. (1996) zeigen übereinstimmend mit den Befunden von Bates et al. (1994), dass auch Kinder mit sehr kleinem Gesamtwortschatz neben Substantiven Wörter anderer Kategorien produzieren. Daraus folgert sie, dass Spracherwerb einerseits begrifflich-semantisch über Substantive und andererseits sprachspezifisch über Verben und Funktionswörter erfolgt.

Für den Wortgebrauch und das Wortlernen bemerken Bloom et al. (1993), dass weniger die Wortarten als vielmehr das Prinzip der Relevanz maßgeblich sei. Ob ein Kind ein Wort lernt, hängt also hauptsächlich davon ab, ob es für das Kind wichtig ist, dieses Wort äußern zu können und nicht davon, ob das betreffende Wort z.B. ein Substantiv oder ein Verb ist.

Stern und Stern (1922) verbinden das Auftreten bestimmter Wortarten mit sogenannten „Denkstadien“. Sie unterscheiden Substanz-, Aktions- und Merkmals- und Relationsstadium, die das Kind als Etappen seines intellektuellen Lebens durchläuft. 
Im Substanzstadium überwiegt die Erfahrung von Personen und Objekten (entspricht der Wortart der Substantive), im Aktionsstadium die Erfahrung der an Objekten und Personen vollziehbaren Handlungen bzw. Vorgänge (die Anzahl an Verben verfünffacht sich). Im Relations- und Merkmalsstadium steht die Erfahrung der Merkmale der Objekte und der zwischen ihnen bestehenden Beziehungen im Vordergrund (Adjektive, Adverbien, Pronomen, Präpositionen und Numeralia).

Für die Wortartenentwicklung im Deutschen kann zusammenfassend festgehalten werden, dass die sozialen, relationalen und lautmalerischen Wörter der frühen Phase zunehmend durch andere Wortarten (Substantive, Verben, Adjektive, Pronomen und Partikel) ersetzt werden. Dabei gibt es für einige Wortarten spezifische Entwicklungsmuster, die mit den Befunden zur Wortartenentwicklung in der englischen Sprache vergleichbar sind: Substantive zeigen einen frühen Zuwachs mit anschließendem Rückgang. Verben entstehen später als Substantive nehmen aber konstant zu. Für Pronomen, Artikel und andere Wortarten mit rein grammatischer Funktion kann ein später massiver Anstieg im Übergang von der Ein- zur Zweiwortphase beobachtet werden. Mit drei Jahren ist die Komposition des Lexikons mit der Zusammensetzung von erwachsenen Sprechern vergleichbar (Kauschke, 2003).

Gibt es bestimmte Wortarten, die generell oder in einem spezifischen Zeitfenster des frühen Spracherwerbs sehr gute Indikatoren für den Sprachentwicklungsstand sind und sich somit zur Vorhersage späterer Sprachleistungen in besonderem Maße eignen? Und sind Abweichungen in der qualitativen Wortschatzentwicklung aussagekräftig für späteres Sprachoutcome? Ist zum Beispiel ein Kind, welches von Anfang an parallel alle Wortarten erwirbt, benachteiligt in seiner späteren sprachlichen Entwicklung bis ins Grundschulalter gegenüber einem Kind, welches sich zunächst vorwiegend auf den Erwerb von Substantiven konzentriert? Dies alles sind Fragen, die bisher überwiegend unbeantwortet sind.

Jüngere Forschung zu semantischer und syntaktischer Entwicklung normal entwickelter Kinder lenkt die Aufmerksamkeit auf die Wortart der Verben. Immer mehr Sprachentwicklungsforscher betonen die zentrale Rolle der Verben im Spracherwerb und Sprachgebrauch, da Verben spezifische konzeptuelle Rollen beschreiben (wie z.B. „der Geber“, „,der Nehmer“ beim Verb „geben“), welche den Rahmen für die Einordnung anderer Wortklassen ins linguistische System bilden. In diesem Sinne stellen Verben einen Stimulus für die frühe Grammatikentwicklung dar. Sie können als Schlüssel zum Spracherwerb innerhalb des kognitiv-linguistischen Rahmens betrachtet werden (Tomasello, 1992).

Behrens $(1999,2002)$ bewertet den Aufbau des Verbenlexikons als höchst relevanten Prozess, da Verben als Bindeglied zwischen lexikalischer und grammatischer Entwicklung 
fungieren. Ein ausreichend großes Verbenlexikon stellt somit die Grundlage für weitere Sprachentwicklungsfortschritte dar. Marchman und Bates (1994) heben hervor, dass die Verwendung von Verben und die Entwicklung der Verbmorphologie sowie die weitere grammatische Entwicklung abhängig sind von dem Erreichen einer bestimmten kritischen Masse an Wörtern im expressiven Wortschatz (,critical mass hypothesis“). Eine kritische Masse an Informationen kann die Fähigkeit zum Abstrahieren und Generalisieren kognitiver Schemata beeinflussen. Strukturiertes Material ist einfacher zu lernen und Schemata fungieren als Abrufhilfen auch für schlecht verankertes verbales Material, was den nicht linearen Zusammenhang zwischen lexikaler und grammatischer Entwicklung erklären mag.

Studien an Kindern mit „specific language impairment“ (SLI) ergaben, dass sich diese Kinder von normal entwickelten Kindern insbesondere in Bezug auf die Größe und Diversifizität ihres Verbenlexikons unterschieden sowie in ihrer Fähigkeit neue Verben zu erlernen und in ihre Spontansprache zu integrieren (z.B. Conti-Ramsden, 2003 und King \& Fletcher, 1993). So benötigten Kinder mit SLI den doppelten Input an neuen Verben, um diese im Vergleich zur zwei Jahre jüngeren Kontrollgruppe (Matching nach Größe des Verbenlexikons) in ihren expressiven Wortschatz aufzunehmen (Conti-Ramsden, 2003). Kinder mit SLI verwendeten deutlich mehr Substantive als Verben in ihrer expressiven Sprache und machten mehr semantische Fehler beim Gebrauch von Verben (Kelly, 1997). Verbenerwerb- und gebrauch sowie verbgebundene grammatische Fähigkeiten scheinen bei Kindern mit SLI mit besonderen Schwierigkeiten verbunden zu sein, die bis ins Schulalter persistieren (Paul, 1993).

Was aber unterscheidet Verben von Substantiven und was macht erstgenannte zu einer so schwierigen Lernaufgabe insbesondere für Kinder mit spezifischer Sprachstörung?

Der Erwerb von Verben stellt eine schwerere kognitive Aufgabe dar als der Substantiverwerb, denn zum einen sind Verben nicht immer im Zusammenhang mit einem Ort, Objekt bzw. einer Person beobachtbar und sie sind oft nur für eine kurze Zeitspanne zu beobachten. Zum anderen stellt die Statusveränderung, die durch Verben beschrieben wird, generell eine höhere kognitive Anforderung für das Kind dar. Auch erfährt ein Kind als Beobachter einer Handlung häufig ein anderes Feedback als wenn es selber die Handlung ausführt.

Marchman und Bates (1994) betonen, dass Verben besonders gut die morphologische Entwicklung vorhersagen. Hierbei bezeichnen die Autoren den Umfang des expressiven Verbenlexikons als stärksten und konsistentesten Prädiktor für die morphologische Entwicklung. Die hohe prädiktive Validität des frühen expressiven Verbenlexikons hat 
insbesondere Gültigkeit ab dem Vorliegen einer kritischen Masse im expressiven Gesamtwortschatz.

Für das Verbenlexikon sind substanzielle Zusammenhänge zu späterem Sprachoutcome und bedeutsame Varianzaufklärungsbeiträge für spätere Sprachleistungen $\mathrm{zu}$ erwarten. Fraglich ist jedoch, ob sie als Teilmenge des frühen Gesamtwortschatzes zusätzliche substanzielle Varianzaufklärungen z.B. für die spätere Grammatikentwicklung über den Varianzaufklärungsbeitrag des gesamten expressiven Wortschatzumfanges hinaus erbringen und ob höhere Zusammenhänge zu späterem Sprachoutcome bestehen als für den gesamten Wortschatzumfang.

Neben der Betrachtung des frühen Verbenwortschatzes nach Verbarten wie Modalverben (z.B. „dürfen“, „,können“, „müssen“) und Hilfsverben bzw. Auxiliarverben (z.B. „haben“, „sein“), lassen sich die Verben mit Hinblick auf ihre Valenz also ihren Wert analysieren. Valenz ist hierbei definiert als die Information, welche die gewünschte syntaktische Umgebung eines Wortes spezifiziert (Dijkstra \& Kempen, 1993). Ein Verb niedriger Valenz verlangt nur ein Subjekt zur Bildung eines vollständigen Satzes (z.B. ,ich laufe“, „ich spiele“, „,ich rutsche“) und wird auch als Vollverb bezeichnet (Dudenredaktion, 2006). Die Valenz eines Verbs steigt mit der Anzahl der Konstituenten, die dieses zur vollständigen Satzbildung verlangt. So verfügen Modalverben über eine höhere Valenz als Vollverben, denn sie verlangen neben dem Subjekt nach dem Infinitiv eines weiteren Verbs (z.B. „ich kann malen“). Andere Verben verlangen nach einem Subjekt und Akkusativobjekt (z.B. zumachen: „,ich mache die Tür zu“). Höherwertige Verben sind verbunden mit der Verwendung längerer Sätze. Dementsprechend wird erwartet, dass die Summe höherwertige Verben im frühen expressiven Wortschatz von Kindern ein besonders guter Indikator für den Stand der grammatischen Entwicklung ist.

Es wird erwartet, dass die Summe der höherwertigen Verben substanzielle Varianzaufklärungsbeiträge für späteres Sprachoutcome (insbesondere für die grammatischen Leistungen) leistet und in bedeutsamen Zusammenhang zu den späteren Sprachleistungen steht. Offen ist, ob der Bestand an höherwertigen Verben $\mathrm{zu}$ zusätzlichen Varianzaufklärungbeiträgen über den gesamten Wortschatzumfang hinaus führt und ob die Informationen, die in dieser Teilmenge des gesamten Wortschatzes stecken, so relevant für späteres Sprachoutcome sind, dass sie in höherem Zusammenhang zu selbigem stehen als der gesamte frühe Wortschatzumfang.

Kauschke (2003) weist auf die Notwendigkeit der differentiellen Diagnostik lexikalischer Prozesse hin. Neben den Aussagen über lexikalische Inventarbeschränkungen und 
modalitätsspezifische Auffälligkeiten (Rezeption vs. Produktion) sei die Feststellung wortartspezifischer Defizite besonders relevant. Die große Bedeutung der Wortart Verben für den weiteren Spracherwerb macht die Frage nach dem Bestehen eines Verbendefizites mit Implikationen für die weitere Sprachentwicklung zu einer Frage von besonderem klinischem Interesse. Untersucht werden soll, ob ein frühes Verbendefizit, also das vollständige Fehlen der Wortart Verben, in signifikantem Zusammenhang zu späterem Sprachoutcome steht.

Für die weiteren Wortarten wie z.B. Adjektive und Pronomen sind der aktuellen Forschungsliteratur keine Aussagen in Bezug auf Zusammenhänge zu späterem Sprachoutcome bzw. prädiktiver Validität für späteres Sprachoutcome zu entnehmen. Erwartet werden aufgrund des hohen grammatischen Bedeutungsgehaltes der Wortarten Adjektive, Zahladjektive, Pronomen/Artikel sowie Frageadverbien substanzielle Zusammenhänge zu späterem Sprachoutcome. Das Bestehen zusätzlicher signifikanter Varianzaufklärungsbeiträge (über den Gesamtwortschatzumfang hinaus) sowie höherer Zusammenhänge zu späterem Sprachoutcome als für den Gesamtwortschatz sind fraglich.

Für die Wortart der Substantive liegt aus der Literatur der Hinweis vor, dass sie das frühe Lexikon von Kindern dominieren. Dieses spiegelt sich auch in allen Erhebungsverfahren für den frühen expressiven Wortschatz wider. So enthält der ELAN z.B. 162 Substantive im Vergleich zu 88 Nicht-Substantiven. Da Substantive also den größten Anteil des Gesamtwortschatzes bilden, sind die Informationen, die diese Wortart repräsentiert (im Unterschied zu den anderen Wortarten), gut durch den Gesamtwortschatz vermittelt. Zudem finden Substantive überwiegende Verwendung als Einwortäußerungen (im frühen Alter) und sind somit wahrscheinlich der schlechtere Indikator für grammatische Entwicklung im Vergleich zu den anderen erwähnten Wortarten. Es werden für die Wortart der Substantive weder substanzielle zusätzliche Varianzaufklärungsbeiträge über den Gesamtwortschatzumfang hinaus noch höhere Zusammenhänge zu späterem Sprachoutcome als für den gesamten Wortschatzumfang erwartet und die Wortart wird folglich nicht in die weiteren Berechnungen einbezogen.

\subsubsection{Ergebnisse}

Das methodische Problem der kleinen Itemmengen (bezogen auf die Wortarten als Teilmengen des Gesamtwortschatzes) galt für alle Wortarten. So verfügte die Wortart Frageadverbien lediglich über drei Items und die Wortart Zahladjektive über fünf Items im ELAN. Dieser Tatbestand machte aus methodischen Gründen den Nachweis signifikanter Zusammenhänge zu späterem Sprachoutcome bei üblichem Signifikanzprüfungsniveau von 
5\% relativ unwahrscheinlich (siehe Reliabilitätsproblem). In diesem explorativen abschließenden Kapitel der Arbeit interessierte im ersten Schritt vor allem, ob überhaupt signifikante Zusammenhänge der Teilmengen zu späterem Sprachoutcome auch für so kleine Itemmengen nachzuweisen waren. Hierfür wurde eine liberalere Handhabung bzgl. des Signifikanzniveaus für die Korrelationsanalysen gewählt und dieses auf $\alpha=.10$ (bei zweiseitiger Testung) festgelegt. Im zweiten Schritt wurden für die Wortarten mit signifikanten Zusammenhängen zu späteren Sprachoutcomevariablen Regressionsanalysen gerechnet, um zu prüfen, ob zusätzliche signifikante Varianzaufklärungen über den gesamten Wortschatzumfang hinaus für die entsprechenden Sprachoutcomes vorlagen. Im dritten Schritt wurden dann bestehende Korrelationsdifferenzen (bei höherer Korrelation für die Teilmenge der Wortarten mit späterem Sprachoutcome als für den gesamten Wortschatzumfang) auf Signifikanz geprüft.

Als Variablen für das Sprachoutcome gingen für den Wortschatz zu $\mathrm{t}_{1}$ und $\mathrm{t}_{2}$ die Rohwerte im AWST 3-6 (WS) und zu t 3 die Rohwerte des Untertests „Wortschatz“ aus dem HAWIK III (HAWIK WS) in die Korrelations- und Regressionsanalysen ein. Für die rezeptiven grammatischen Fähigkeiten fungierten $\mathrm{zu} \mathrm{t}_{1}$ und $\mathrm{t}_{2}$ die Rohwerte des Untertests „Verstehen von Sätzen“ aus dem SETK 3-5 (VS) und zu t $\mathrm{z}_{3}$ die Rohwerte im Untertest „Verstehen grammatischer Strukturformen“ aus dem HSET (HSET VS) als Variablen für die weiteren Berechungen. Bezogen auf die expressiven Leistungen fungierten die Rohwerte im Untertest „morphologische Regelbildung“ (SETK 3-5; MR) zu t $\mathrm{t}_{1}$ und $\mathrm{t}_{2}$ sowie die Rohwerte aus dem Untertest „Plural-Singular-Bildung“ (HSET; PS) zu t 3 als Variablen in den Korrelations- und Regressionsanalysen. Für das Satzgedächtnis wurden die Rohwerte im Untertest „Satzgedächtnis“ (SETK 3-5; SG) zu t $\mathrm{t}_{1}$ und $\mathrm{t}_{2}$ sowie die Rohwerte im Untertest „Imitation grammatischer Strukturformen“ (HSET; IS) als Variablen in den Korrelations- und Regressionsanalysen verwendet.

Das frühe Verbeninventar des ELANs (31 Verben) wurde in Vollverben und Verben höherer Valenz eingeteilt. Diese Analyse aller Verben zu to ergab, dass 12 von 31 Verben neben dem Subjekt ein weiteres Satzobjekt verlangen. Hierbei wurde das Verb „machen“ ausgeschlossen, da es ein all purpose verb ist, welches stellvertretend für viele andere Verben im jungen Alter verwendet wird. Als Variable „Vollverben“ fungierte die Summe der Vollverben aus dem ELAN (Max = 18) und als „,valente Verben“ die Summe der höherwertigen Verben $(\mathrm{Max}=12)$ aus dem ELAN. Zudem wurde die Summe aller Verben im ELAN $($ Max $=31)$ sowie die Summe aller Hilfs- und Modalverben $($ Max $=8)$ im ELAN mit 
den Variablen zum späteren Sprachoutcome korreliert und in die Regressionsanalysen eingefügt. Die Variable „Verbendefizit“ verfügte über den Wert 0, wenn doch mindestens ein Verb im ELAN als gesprochenes Wort von den Eltern angekreuzt worden war und der Wert 1 wurde vergeben, wenn kein Verb im ELAN angegeben war.

Für die anderen Wortarten fungierten die Summe der Adjektive im ELAN mit Ausnahme der Zahlenadjektive (Max = 11), die Summe der Zahlenadjektive („Mengen“) im ELAN (Max = 5), die Summe aller Pronomen und Artikel im ELAN (Max = 14) und die Summe der Frageadverbien im ELAN (Max = 3) als Variablen für die weiteren Berechnungen. Die Summe der sonstigen Wörter (,Sonstige“) im ELAN (Max =24), welche überwiegend aus Interjektionen (z.B. „Hallo“) sowie relationalen Wörtern (z.B. „ab“, „,weg“, „da“) besteht, wurde zudem als Variable in die Berechnungen eingegeben und repräsentiert insbesondere die ganz frühen Wortarten, die nicht immer eindeutig zuzuordnen sind.

Für alle Variablen mit Ausnahme des Verbendefizits wurden Pearson-Produkt-MomentKorrelationen berechnet und auf Signifikanz geprüft. Für die Variable „Verbendefizit“ wurden punktbiseriale Korrelationen aufgrund des nominalen Skalenniveaus errechnet. In Tabelle 13 sind die Ergebnisse für die Wortart der Verben sowie zusätzlich die Korrelationen für den Prozentrang im ELAN (Gesamtwortschatzumfang) mit den Sprachoutcomevariablen und den einzelnen Wortartenvariablen dargestellt. Die signifikanten Korrelationen für die Wortartenvariablen sind in der Tabelle gelb hinterlegt (zweiseitige Testung), die für den Gesamtwortschatzumfang rot (einseitige Testung). 
Tabelle 13

Zusammenhänge von Wortartenvariablen (Verben) und Gesamtwortschatzumfang mit Sprachoutcome von $t_{0}$ bis $t_{3}$

\begin{tabular}{|c|c|c|c|c|c|c|}
\hline & Alle Verben & Vollverben & $\begin{array}{l}\text { Valente } \\
\text { Verben }\end{array}$ & $\begin{array}{l}\text { Hilfs- und } \\
\text { Modalverbe } \\
\mathrm{n}\end{array}$ & $\begin{array}{l}\text { Verben- } \\
\text { defizit }\end{array}$ & $\begin{array}{l}\text { Gesamt- } \\
\text { wortschatz }^{\text {a }}\end{array}$ \\
\hline $\mathbf{t}_{\mathbf{0}}$ & $\begin{array}{ll}r & (p)\end{array}$ & $\begin{array}{ll}r & (p)\end{array}$ & $\begin{array}{ll}r & (p)\end{array}$ & $\begin{array}{ll}r & (p)\end{array}$ & $\begin{array}{ll}r & (p)\end{array}$ & $\begin{array}{ll}r & (p)\end{array}$ \\
\hline $\begin{array}{l}\text { PR } \\
\text { ELAN }\end{array}$ & $.862(.000)$ & $.863(.000)$ & $.823(.000)$ & $.765(.000)$ & $-.636(.000)$ & - \\
\hline \multicolumn{7}{|l|}{$\mathbf{t}_{1}$} \\
\hline WS & $.303(.064)$ & $.317(.053)$ & $.262(.111)$ & $.288(.079)$ & $-.243(.141)$ & $.382(.009)$ \\
\hline $\begin{array}{l}\text { VS } \\
\text { 3-jähr. }\end{array}$ & $.057 .(782)$ & $.103(.616)$ & $-.064(.756)$ & $-.158(.442)$ & $-.029(.890)$ & $.209(.152)$ \\
\hline $\begin{array}{l}\text { MR } \\
\text { 3-jähr. }\end{array}$ & $-.085(.681)$ & $.004(.984)$ & $-.273(.176)$ & $-.381(.055)$ & $.176(.391)$ & $.090(.331)$ \\
\hline $\begin{array}{l}\text { VS } \\
\text { 4-jähr. }\end{array}$ & $.455(.010)$ & $.482(.006)$ & $.365(.044)$ & .421 & $-.289(.114)$ & $.463(.004)$ \\
\hline $\begin{array}{l}\text { MR } \\
\text { 4-jähr. }\end{array}$ & $.573(.001)$ & $.593(.000)$ & $.525(.002)$ & $.525(.002)$ & $-.527(.002)$ & $.658(.000)$ \\
\hline $\mathrm{SG}$ & $.424(.017)$ & $.414(.021)$ & $.436(.014)$ & $.453(.011)$ & $-.318(.082)$ & $.424(.009)$ \\
\hline \multicolumn{7}{|l|}{$\mathbf{t}_{2}$} \\
\hline WS & $.420(.002)$ & $.433(.001)$ & $.396(.003)$ & $.329(.015)$ & $-.315(.020)$ & $.515(.000)$ \\
\hline VS & $.302(.027)$ & $.339(.012)$ & $.228(.097)$ & $.252(.066)$ & $-.286(.036)$ & $.364(.003)$ \\
\hline MR & $.234(.089)$ & $.219(.112)$ & $.259(.059)$ & $.303(.026)$ & $-.153(.270)$ & $.376(.003)$ \\
\hline $\mathrm{SG}$ & $.323(.030)$ & $.318(.033)$ & $.308(.039)$ & $.330(.027)$ & $-.217(.152)$ & $.432(.002)$ \\
\hline \multicolumn{7}{|l|}{$\mathbf{t}_{3}$} \\
\hline WS & $.175(.215)$ & $.153(.279)$ & $.245(.080)$ & $.170(.229)$ & $-.078(.585)$ & $.276(.024)$ \\
\hline HSET VS & $.183(.190)$ & $.170(.224)$ & $.197(.158)$ & $.162(.248)$ & $-.028(.840)$ & $.215(.061)$ \\
\hline PS & $.134(.340)$ & $.147(.294)$ & $.119(.394)$ & $.011(.940)$ & $-.033(.817)$ & $.300(.015)$ \\
\hline IS & $.235(.091)$ & $.241(.082)$ & $.225(.105)$ & $.237(.088)$ & $.001(.994)$ & $.384(.002)$ \\
\hline
\end{tabular}

Anmerkungen. $\mathrm{t}_{0}=$ Messzeitpunkt 2001, $\mathrm{t}_{1,2,3}=$ Messzeitpunkt 1 bis 3, PR ELAN $=$ Prozentrang im ELAN, WS = Wortschatz, VS = rezeptive grammatische Fähigkeiten gemessen mit dem Untertest „Verstehen von Sätzen“ (VS) aus dem SETK 3-5, MR = expressive grammatische Fähigkeiten gemessen mit dem Untertest „Morphologische Regelbildung“ (MR) aus dem SETK 3-5, SG = Satzgedächtnis, HSET VS = rezeptive grammatische Fähigkeiten gemessen mit dem HSET VS, PS = expressive grammatische Fähigkeiten gemessen mit dem Untertest „Plural-Singular-Bildung“ (PS) aus dem HSET, IS = Satzgedächtnis gemessen mit dem Untertest „Imitation grammatischer Strukturformen“ (IS) aus dem HSET, $r=$ Pearson Produkt Moment Korrelationen, $p=$ Fehlerwahrscheinlichkeit

${ }^{a}$ einseitige Testung

Tabelle 14 veranschaulicht die Ergebnisse für die anderen Wortartvariablen (außer Verben) sowie zusätzlich die Korrelationen für den Prozentrang im ELAN 
(Gesamtwortschatzumfang) mit den Sprachoutcomevariablen und den einzelnen Wortartenvariablen.

Die signifikanten Korrelationen für die Wortartenvariablen sind in der Tabelle gelb (zweiseitige Testung) hinterlegt, die für den Gesamtwortschatzumfang rot (einseitige Testung).

Tabelle 14

Zusammenhänge weiterer Wortartenvariablen (außer Verben) und des Gesamtwortschatzumfangs mit Sprachoutcome von $t_{0}$ bis $t_{3}$

\begin{tabular}{|c|c|c|c|c|c|c|}
\hline & Adjektive & $\begin{array}{l}\text { Pronomen } \\
\text { und Artikel }\end{array}$ & $\begin{array}{l}\text { Zahl- } \\
\text { adjektive } \\
\text { (Mengen) }\end{array}$ & $\begin{array}{l}\text { Frage- } \\
\text { adverbien }\end{array}$ & Sonstige & $\begin{array}{l}\text { Gesamt- } \\
\text { wortschatz }^{\mathrm{a}}\end{array}$ \\
\hline $\mathbf{t}_{0}$ & $\begin{array}{ll}r & (p)\end{array}$ & $\begin{array}{ll}r \quad(p) \\
\end{array}$ & $r \quad(p)$ & $\begin{array}{ll}r \quad(p) \\
\end{array}$ & $\begin{array}{ll}r & (p)\end{array}$ & $\begin{array}{ll}r & (p)\end{array}$ \\
\hline $\begin{array}{l}\text { PR } \\
\text { ELAN }\end{array}$ & $.853(.000)$ & $.795(.000)$ & $.756(.000)$ & $.583(.000)$ & $.827(.000)$ & - \\
\hline \multicolumn{7}{|l|}{$\mathbf{t}_{1}$} \\
\hline WS & $.308(.060)$ & $.248(.133)$ & $.247(.136)$ & $.210(.206)$ & $.363(.025)$ & $.382(.009)$ \\
\hline $\begin{array}{l}\text { VS } \\
\text { 3-jähr. }\end{array}$ & $.223 .274)$ & $-.073(.725)$ & $.390(.049)$ & $-.259(.202)$ & $.156(.447)$ & $.209(.152)$ \\
\hline $\begin{array}{l}\text { MR } \\
\text { 3-jähr. }\end{array}$ & $-.107(.602)$ & $.097(.637)$ & $.105(.611)$ & $.060(.770)$ & $-.118(.565)$ & $.090(.331)$ \\
\hline $\begin{array}{l}\text { VS } \\
\text { 4-jähr. }\end{array}$ & $.465(.008)$ & $.532(.002)$ & $.602(.000)$ & $.343(.059)$ & $.577(.001)$ & $.463(.004)$ \\
\hline $\begin{array}{l}\text { MR } \\
\text { 4-jähr. }\end{array}$ & $.561(.001)$ & $.545(.002)$ & $.556(.001)$ & $.356(.049)$ & $.666(.000)$ & $.658(.000)$ \\
\hline $\mathrm{SG}$ & $.391(.030)$ & $.409(.023)$ & $.283(.122)$ & $.402(.025)$ & $.377(.037)$ & $.424(.009)$ \\
\hline \multicolumn{7}{|l|}{$\mathbf{t}_{2}$} \\
\hline WS & $.441(.001)$ & $.391(.003)$ & $.342(.011)$ & $.267(.051)$ & $.444(.001)$ & $.515(.000)$ \\
\hline VS & $.309(.023)$ & $.298(.029)$ & $.308(.023)$ & $.164(.236)$ & $.388(.004)$ & $.364(.003)$ \\
\hline MR & $.203(.140)$ & $.247(.072)$ & $.283(.038)$ & $.297(.029)$ & $.287(.036)$ & $.376(.003)$ \\
\hline SG & $.252(.095)$ & $.278(.064)$ & $.289(.054)$ & $.405(.006)$ & $.191(.210)$ & $.432(.002)$ \\
\hline \multicolumn{7}{|l|}{$\mathbf{t}_{3}$} \\
\hline WS & $.189(179)$ & $.148(.295)$ & $.132(.350)$ & $.289(.038)$ & $.185(.189)$ & $.276(.024)$ \\
\hline HSETVS & $.178(.202)$ & $.179(.200)$ & $.201(.149)$ & $.180(.196)$ & $.129(.359)$ & $.215(.061)$ \\
\hline PS & $.133(.344)$ & $.105(.456)$ & $.114(.418)$ & $-.024(.864)$ & $.146(.296)$ & $.300(.015)$ \\
\hline IS & $.228(.101)$ & $.259(.061)$ & $.213(.126)$ & $.292(.034)$ & $.216(.120)$ & $.384(.002)$ \\
\hline
\end{tabular}

Anmerkungen. $\mathrm{t}_{0}=$ Messzeitpunkt 2001, $\mathrm{t}_{1,2,3}=$ Messzeitpunkt 1 bis 3, PR ELAN $=$ Prozentrang im ELAN, WS = Wortschatz, VS = rezeptive grammatische Fähigkeiten gemessen mit dem Untertest „Verstehen von Sätzen“ (VS) aus dem SETK 3-5, MR = expressive grammatische Fähigkeiten gemessen mit dem Untertest „Morphologische Regelbildung“ (MR) aus dem SETK 3-5, SG = Satzgedächtnis, HSET VS = rezeptive grammatische Fähigkeiten gemessen mit dem HSET VS, PS = expressive grammatische Fähigkeiten gemessen mit dem Untertest „Plural-Singular-Bildung“ (PS) aus dem HSET, IS = Satzgedächtnis gemessen mit dem Untertest „Imitation grammatischer Strukturformen“ (IS) aus dem HSET, $r=$ Pearson Produkt Moment Korrelationen, $p=$ Fehlerwahrscheinlichkeit

${ }^{a}$ einseitige Testung 
Für die Wortartenvariablen konnten $\mathrm{zu}$ minimal 4 bis höchstens 10 Sprachoutcomevariablen signifikante Korrelationen errechnet werden. Die Wortartenvariablen mit signifikanten Korrelationen zu den meisten Sprachoutcomevariablen waren die Summe der Hilfs- und Modalverben $(r=.24-.52$; signifikante Korrelationen zu 10 Sprachoutcomevariablen) sowie die Summe aller Verben $(r=.23$ - .57; signifikante Korrelationen zu 9 Sprachoutcomevariablen). Für den Prozentrang im ELAN bestanden im Vergleich signifikante Korrelationen zu 11 der 14 erhobenen Sprachoutcomevariablen in der Höhe von .28 - .66. Die weiteren Wortartenvariablen (mit Ausnahme der Variable Verbendefizit) korrelierten mit 7 bis 8 der Sprachoutcomevariablen signifikant. Die Korrelationen erreichten für die weiteren Wortarten Werte von .23 - .67. Die meisten signifikanten Korrelationen bestanden mit den Sprachoutcomevariablen $\mathrm{zu}_{1}$, die sich auf die Stichprobe der vierjährigen Kinder bezogen und mit den Sprachoutcomevariablen zu $t_{2}$.

Für den Untertest MR zu t $\mathrm{t}_{1}$ bezogen auf die Stichprobe der dreijährigen Kinder bestand keine signifikante Korrelation mit dem Prozentrang im ELAN $(r=.09, p=.661)$ wohl aber mit der Summe der Hilfs- und Modalverben $(r=-.381 ; p=.055)$. Für den Untertest VS ergab sich eine signifikante Korrelation mit der Summe der Zahladjektive $(r=.39 ; p=.049)$ nicht aber mit dem Prozentrang im ELAN ( $r=.209 ; p=.305)$.

Tendenziell höhere signifikante Korrelationen der Wortartenvariablen mit den Sprachoutcomevariablen im Vergleich zum Prozentrang im ELAN bestanden für die Summe der Vollverben (VS dreijährige Kinder $\mathrm{zu} \mathrm{t}_{1}$ ), die Summe valenter Verben $\left(S G \mathrm{zu} \mathrm{t}_{1}\right)$, die Summe der Hilfs- und Modalverben ( $\mathrm{SG} \mathrm{zu} \mathrm{t}_{1}$ ), die Summe der Adjektive sowie die Summe der Pronomen/Artikel (VS vierjährige Kinder $\mathrm{zu} \mathrm{t}_{1}$ ), die Summe der Zahladjektive (VS vierjährige $\mathrm{zu} \mathrm{t}_{1}$ ), die Summe der Frageadverbien (HAWIK WS $\mathrm{zu}_{3}$ ) und die Summe sonstiger Wörter (VS und MR der vierjährigen zu $\mathrm{t}_{1}$ und VS zu t $\mathrm{t}_{2}$ ). Insgesamt korrelierten die Wortartenvariablen mit Ausnahme der Variable Verbendefizit signifikant hoch positiv mit dem Prozentrang im ELAN $(r=.58-.86 ; p=.000)$. Die Variable Verbendefizit korrelierte mit dem Prozentrang im ELAN in Höhe von -.636 $(p=.000)$. Darüber hinaus korrelierte sie lediglich mit vier Outcomevariablen (MR und $\mathrm{SG}$ der vierjährigen Kinder zu $\mathrm{t}_{1}$ sowie WS und VS zu t $t_{2}$ ). Die Korrelationen der Variable Verbendefizit mit späterem Sprachoutcome beliefen sich mehrheitlich erwartungskonform auf negative Werte. Die signifikanten Korrelationen erreichten Werte von -.29 bis -.53 . 
In den Regressionsanalysen wurde um grundsätzliche methodische Probleme auszuschließen als Vorgehen Forced Entry gewählt, da die Summenwerte für die Wortarten echte Teilmengen des gesamten Wortschatzumfangs (Prozentrang im ELAN) darstellen.

Die beschriebenen Sprachoutcomevariablen wurden als Kriteriumsvariablen in den Regressionsanalysen definiert und als Prädiktorvariable wurde der Prozentrang im ELAN festgelegt. Die nicht aufgeklärten Varianzanteile (standardisierte Residuen) aus allen Regressionsanalysen wurden als neue Kriteriumsvariablen festgelegt und in folgenden einfachen Regressionsanalysen aus den Wortartenvariablen als Prädiktorvariablen vorhergesagt.

Keine der Wortartenvariablen konnte signifikante Varianzaufklärungsbeiträge für die standardisierten Residuen aus den ersten Regressionsanalysen leisten.

Für alle signifikanten Korrelationen von Wortartvariablen mit Sprachoutcome und von Prozentrang im ELAN mit Sprachoutcome wurden die Korrelationsdifferenzen berechnet und bei tendenziell höheren Korrelationen für die Wortartenvariablen auf Signifikanz geprüft.

Für keine der Korrelationsdifferenzen errechnete sich ein signifikantes Ergebnis.

\subsubsection{Diskussion}

Die vielen signifikanten Korrelationen der Wortartenvariablen (vo mittlerer bis hoher Effektstärke) zu späterem Sprachoutcome insbesondere zu $t_{1}$ und $t_{2}$ erstaunen, wenn man die geringen Itemmengen in Betracht zieht, auf die sich diese Aussagen gründen. Für diese zwei Zeitpunkte sind die Korrelationen recht gut mit denen des Gesamtwortschatzes vergleichbar, der z.B. ebenso nicht mit den an der Stichprobe der dreijährigen erhobenen grammatischen Maßen signifikant korreliert. Die signifikante negative Korrelation der Summe aller Hilfsund Modalverben mit den expressiven grammatischen Leistungen der dreijährigen Kinder zu $t_{1}$ sollte hierbei nicht überbewertet werden, da dieses Ergebnis lediglich auf einer Teilstichprobe von 26 Kindern fußt und zudem inhaltlich nicht nachvollziehbar ist. Denn warum sollte eine große Summe an Modal- und Hilfsverben zu schlechteren expressiven grammatischen Leistungen führen? Das abweichende Ergebnis für die rezeptiven grammatischen Leistungen $\mathrm{zu}_{\mathrm{t}}$ für die Stichprobe der dreijährigen Kinder (signifikant positive Korrelation im Unterschied zum Prozentrang im ELAN) ist inhaltlich eher begründbar. Es wird aber auch relativierend betrachtet, da es an einer Teilstichprobe erhoben wurde. Grundsätzlich ist jedoch denkbar, dass gerade die Wortart der Zahlenadjektive das „Verstehen von Mengen“ (Mengenverständnis) eines Kindes repräsentiert und somit 
besondere kognitive Ressourcen für das allgemeine Verständnis von Umwelterfahrungen und Zusammenhängen darstellt. Folglich würde der bedeutsame Zusammenhang zum Untertest „Verstehen von Sätzen“ inhaltlich erklärbar sein.

Die wenigen signifikanten Korrelationen der Wortartenvariablen zum Sprachoutcome zu $\mathrm{t}_{3}$ im Vergleich zum Gesamtwortschatz lassen sich gut durch die höhere Reliabilität der Messung (bezogen auf den Gesamtwortschatz) erklären, welche insbesondere für Zusammenhänge über eine lange Zeitspanne zum Tragen zu kommen scheint.

Insgesamt deuten die hohen und substanziellen Korrelationen der Wortartenvariablen mit dem Prozentrang im ELAN (mittlere Korrelation $=.79$ ) darauf hin, dass der Gesamtwortschatz und seine Teilmengen die Wortarten in sehr engem Zusammenhang stehen und vergleichbare Informationen beinhalten.

Für die Variable Verbendefizit zeigen die Ergebnisse eine tendenzielle Bestätigung der Annahme, das bei Vorliegen solch eines Defizits im Laufe der weiteren Entwicklung schlechtere sprachliche Leistungen zu erwarten sind, denn es lagen mehrheitlich negative Korrelationen vor. Da aber nur zu vier von 14 Sprachoutcomevariablen signifikante Korrelationen berichtet wurden ( $\mathrm{zu}_{\mathrm{t}}$ und $\mathrm{t}_{2}$ ), sollten keine Vorhersagen für spätere Sprachleistungen aus dem Vorliegen bzw. Nicht-Vorliegen eines Verbendefizits für den normalen Spracherwerb abgeleitet werden. Einschränkend muss ergänzt werden, dass diese Aussage lediglich an der spezifischen Altersverteilung dieser Untersuchungsstichprobe geprüft wurde und nicht generalisiert werden darf. Die Ergebnisse lassen nicht den Schluss $\mathrm{zu}$, dass ein Verbendefizit für einen kritischen Alterswert z.B. eine relevante Variable für die Vorhersage späterer Sprachleistungen sein könnte.

Die Ergebnisse der Regressionsanalysen machen deutlich, dass die Teilmengen der Wortarten keine Verbesserung der Vorhersagen späteren Sprachoutcomes aus dem Gesamtwortschatzumfang erlauben. Zudem zeigen die Ergebnisse aus den Prüfungen der Korrelationsdifferenzen, dass keine höheren Zusammenhänge von den Teilmengen der Wortarten zum Sprachoutcome im Vergleich zum Gesamtwortschatzumfang und dem Sprachoutcome bestehen. Erklärt werden kann dieses zum einen durch die höhere Güte der Messung in Bezug auf den Gesamtwortschatz. Denn 250 Items erlauben eine reliablere Vorhersage späterer Leistungen als eine Itemmenge von z.B. 3 oder 31 Items. Die Güte einer Aussage ist abhängig von der Güte ihrer Messung. Dieses lässt zunächst noch keine inhaltlichen Rückschlüsse zu.

Die hohen Zusammenhänge der Wortartenvariablen mit dem Gesamtwortschatzumfang erlauben hingegen eine inhaltliche Interpretation dieser Ergebnisse. Es scheint bei den 
ausgeprägten Zusammenhängen unwahrscheinlich, dass die untersuchten Wortarten als Teilmengen des ganzen Wortschatzes über zusätzliche relevante Informationen für den späteren Spracherwerb verfügen, welche eine besondere Gewichtung einer spezifischen Wortart rechtfertigen würde. Vielmehr scheint sich die Suche nach Variablen anzubieten, die gerade nicht so ausgeprägt mit dem Gesamtwortschatz in Zusammenhang stehen.

Die Ergebnisse sprechen gegen die Wünsche von Klinikern nach noch kürzeren und schnelleren und dabei genauso effektiven Sprachscreenings auch für das frühe Kindesalter. Je geringer die geprüfte Itemmenge ist, umso kleiner ist nun einmal der Ausschnitt von Realität, der erfasst wird, und umso weniger zuverlässig sind die Vorhersagen die man aus diesen Erhebungen insbesondere für große Zeitintervalle ableiten kann. Eine Verkürzung bestehender Erhebungsverfahren wie des ELANs auf die Summe aller Verben z.B. würde die Durchführung für die Eltern und die Auswertung für das klinische Personal zwar erleichtern, steht aber in keinem Verhältnis zu dem Verlust an Informationen, die gerade die gute Mischung des Gesamtwortschatzes zu beinhalten scheint.

Einschränkend muss festgehalten werden, dass es bei der Durchführung der Korrelationsund Regressionsanalysen auf dem Hintergrund der critical mass hypothesis $\mathrm{zu}$ anderen Ergebnissen hätte kommen können, denn erst ab einer kritischen Menge von Einträgen im Lexikon, erhöht sich die Anzahl des Vorkommens bestimmter Wortarten und Marchman und Bates (1994) betonen, dass das Verbenlexikon insbesondere ab dem Vorliegen einer bestimmten kritischen Masse von Wörtern im Gesamtwortschatz über besonders ausgeprägte prädiktive Validität für späteres Sprachoutcome verfügt. Die Prüfung der in Bezug auf die Wortarten formulierten Hypothesen auf dem Hintergrund der critical mass hypothesis war jedoch aufgrund der geringen Stichprobengröße nicht möglich. So wären bei der Wahl eines Wortschatzumfangs von mindestens 50 Wörtern als „critical mass“ zu to lediglich 35 Kinder in der Untersuchungsstichprobe verblieben und die Daten von 38.6\% der Kinder hätten für die Berechnungen zur Prüfung der Hypothesen nicht herangezogen werden können. 


\subsection{Inhalts- und Funktionswörter}

\subsubsection{Theoretischer Hintergrund}

In der Linguistik und Psycholinguistik unterteilt man den Wortschatz sowohl der Erwachsenen- als auch der Kindersprache in Inhalts- und Funktionswörter.

Inhaltswörter tragen mehr zur Bedeutung als zur Struktur eines Satzes bei. Sie verfügen über eine „kontextunabhängige, selbständige lexikalische Bedeutung“ (Bußmann, 2002, S. 111). Die Zuordnung, welche Wortarten zu den Inhaltswörtern gehören wird nicht einheitlich gehandhabt. In überwiegender Übereinstimmung ordnet man Substantive, Adjektive, Vollverben und Adverbien den Inhaltswörtern zu. Uneinigkeit besteht z.B. bezogen auf die Modalverben und Präfixe. Die Anzahl an Inhaltswörtern in einer Sprache ist sehr groß und wird fortlaufend erweitert und angepasst. Deshalb bezeichnet man Inhaltswörter auch als offene Klasse.

Funktionswörter unterstützen die Struktur eines Satzes. Ihre Hauptaufgabe besteht darin, syntaktische Zusammenhänge innerhalb von Sätzen anzugeben. Sie verfügen somit primär über grammatische (anstelle von lexikalischer) Bedeutung und erfüllen syntaktischstrukturelle Funktion. Zu den Funktionswörtern werden im Allgemeinen Pronomina, Artikel, Präpositionen, Konjunktionen und Hilfsverben gezählt. Die Anzahl der Funktionswörter in einer Sprache ist gering (einige Hundert) und bildet eine stabile Sammlung, welche nicht erweitert wird. Aus diesem Grund werden Funktionswörter im Gegensatz zu Inhaltswörtern als geschlossene Klasse bezeichnet (Dijkstra \& Kempen, 1993).

In der frühen Kindheit und hierbei insbesondere in der Phase der Zweiwortäußerungen im Alter von 1;6 bis 2;0 Jahren ist die Kindersprache überfrachtet mit Inhaltswörtern und arm an Funktionswörtern, welche die Beziehungen zwischen den Inhaltswörtern eines Satzes klarstellen. Deshalb wird die kindliche Sprache in dieser frühen Phase auch als „telegraphische Rede“ bezeichnet (z.B. Brown, 1973). Diese telegraphische Rede ist noch so verkürzt, dass nur die außersprachlichen Informationen wie z.B. die Kenntnis der Bezugssituation das Verständnis der kindlichen Äußerungen ermöglichen.

Grimm, Doil, Müller und Wilde (1996) untersuchten 105 deutschsprachige Kindern im Alter von 16 bis 30 Monaten und stellten sowohl für die Inhalts- als auch für die Funktionswörter signifikante alterskorrelierte Anstiege in der Benennungshäufigkeit fest. In Übereinstimmung mit den Daten von Bates et al. (1994) konnte gezeigt werden, dass der Spracherwerb sowohl über Inhaltswörter als auch über Funktionswörter erfolgt, wobei die Zusammensetzung des kindlichen Lexikons abhängig von der Wortschatzgröße ist und der Anteil an Funktionswörtern erst bei einem Gesamtwortschatz von etwa 200 Worten deutlich 
zunimmt. Kauschke (2003) sieht den späten massiven Anstieg an Funktionswörtern in direktem Zusammenhang mit einem Entwicklungssprung beim Übergang von der Einwortphase in die kombinatorischen Äußerungen. Conboy und Thal (2006) konnten anhand von Studien mit bilingual aufwachsenden Kindern zudem zeigen, dass individuelle Unterschiede im Verhältnis von offenen und geschlossenen Wortklassen abhängig vom Wortschatzzuwachs in der gleichen Sprache sind und nicht vom gesamten zur Verfügung stehenden Wortschatz (in mehreren Sprachen) abhängen.

Das Fehlen von Funktionswörtern bzw. die Dominanz von Inhaltswörtern in den spontansprachlichen Äußerungen wird außer als normales Phänomen des frühen Spracherwerbs auch in den Neurowissenschaften für den Verlust von sprachlichen Fähigkeiten nach abgeschlossenem Spracherwerb (Aphasie) beschrieben. So ist die „telegraphische Sprache“ ein Kardinalsymptom der Broca Aphasie. Die sprachlichen Äußerungen von Patienten mit einer Broca Aphasie sind vor allem dadurch gekennzeichnet, dass sie überwiegend aus Inhaltswörtern bestehen und fast keine Funktionswörter enthalten (Agrammatismus). Dieses gemeinsame „Verschwinden“ aller Funktionswörter bei einer bestimmten neurologischen Funktionsstörung liefert auch die psychologische Rechtfertigung dafür, die Funktionswörter in einer einzigen syntaktischen Klasse zu gruppieren (Miller, 1991).

Betrachtet man die normale Sprache bezogen auf Versprecher, wird deutlich, dass sich Versprecher überwiegend innerhalb einer Kategorie (Inhaltswörter vs. Funktionswörter) vollziehen. So kann ein Inhaltswort die Laute eines anderen Inhaltswortes einfärben wie z.B. „das Glatz platzt“. Ein Inhaltswort wird aber nicht die Laute eines Funktionswortes einfärben (z.B. „dlas Glas platzt“). Zimmer (1988) schließt, dass Inhalts- und Funktionswörtern in zwei Schritten in geplante Äußerungen eingesetzt werden, wobei die Inhaltswörter im ersten Schritt eingeplant werden, da die Funktionswörter von den Inhaltswörtern abhängen (z.B. Artikel) und erst nach deren Feststehen geplant werden können.

Schlussfolgernd ist festzuhalten, dass Funktionswörter und Inhaltswörter in engem Zusammenhang stehen, wobei Inhaltswörter sowohl bezüglich Sprechplanung als auch Spracherwerb zeitlich vorrangig sind. Funktionswörter scheinen einen höheren Anspruch an Spracherwerb und -verarbeitung zu stellen, da sie zum einen später erworben werden und zum anderen leichter „verloren werden“, wie die Analyse von Sprachdaten hirnorganisch erkrankter Menschen zeigt.

Auch erscheint die kognitive Aufgabe reine Bedeutungswörter zu speichern, abzurufen und adäquat zu verwenden eine leichtere zu sein als die Aufgaben, ein komplexes Regelwerk, 
welches die Relationen dieser Bedeutungswörter zueinander spezifiziert, korrekt zu erwerben, $\mathrm{zu}$ speichern und anzuwenden. Ein expressiver Wortschatz der aus besonders vielen Funktionswörtern im Verhältnis zu Inhaltswörtern besteht, sollte Indikator für eine besonders fortgeschrittene grammatische Entwicklung sein.

Erwartet wird, dass sowohl Inhalts- als auch Funktionswörter in einem bedeutsamen Zusammenhang zu späterem Sprachoutcome stehen. Da es in diesem Kapitel um die Suche nach Sprachmaßen geht, die zusätzlich zum gesamten Wortschatzumfang Varianzaufklärung für späteres Sprachoutcome leisten bzw. in höherem Zusammenhang als der Gesamtwortschatz zu späteren Sprachleistungen stehen, werden die Inhaltswörter nicht in die weiteren Berechnungen eingehen. Der theoretische Hintergrund macht deutlich, dass Inhaltswörter den größten Anteil am frühen kindlichen Wortschatz einnehmen. Dieses findet sich in den Erhebungsverfahren wieder. So besteht die Wortschatzcheckliste im ELAN aus 223 Inhaltswörtern und nur 25 Funktionswörtern (2 Items konnten nicht eindeutig zugeordnet werden). Der Gesamtwortschatz repräsentiert im Wesentlichen die Informationen für die spätere Sprachentwicklung, die in den Inhaltswörtern enthalten sind. Bedeutsame Verbesserungen der Vorhersagen späteren Sprachoutcomes aus dem Gesamtwortschatz sowie höhere Zusammenhänge zu späteren sprachlichen Leistungen im Vergleich zum Gesamtwortschatz könnten hingegen die Funktionswörter zum einen aufgrund ihrer Unterrepräsentiertheit im Gesamtwortschatz und zum anderen aufgrund ihrer primär grammatischen Funktion haben. Auch für das Verhältnis von Funktions- zu Inhaltswörtern könnten höhere Zusammenhänge zum späteren Sprachoutcome sowie zusätzliche Varianzaufklärung späteren Sprachoutcomes über den Gesamtwortschatz hinaus bestehen.

\subsubsection{Ergebnisse}

Analog zum in 7.1.2 beschriebenen Vorgehen wurden zunächst die Korrelationen der Summe an Funktionswörtern im ELAN (Max = 25) sowie die Korrelationen des Quotienten aus der Summe aller Funktionswörter und der Summe aller Inhaltswörter im ELAN (QFI) mit den Sprachoutcomevariablen (vgl. 7.1.2) auf Signifikanz geprüft (zweiseitige Testung; $\alpha=.10$ ). Zum besseren Verständnis für die Diskussion der Ergebnisse stellt Tabelle 15 zusätzlich die Korrelationen des Prozentranges im ELAN (einseitige Testung) mit den Sprachoutcomevariablen und der Variablen QFI sowie der Summe aller Funktionswörter dar. Signifikante Korrelationen sind für den Prozentrang im ELAN rot hinterlegt; für den QFI sowie die Summe aller Funktionswörter sind diese gelb hinterlegt. 
Tabelle 15

Zusammenhänge von Funktionswörtern, dem Verhältnis von Funktions- und Inhaltswörtern sowie dem Gesamtwortschatzumfang mit Sprachoutcome von $t_{0}$ bis $t_{3}$

\begin{tabular}{|c|c|c|c|}
\hline & Funktionswörter & $\begin{array}{l}\text { Verhältnis von } \\
\text { Funktions- zu } \\
\text { Inhaltswörtern }\end{array}$ & $\begin{array}{l}\text { Gesamtwortschatz } \\
\text { (PR ELAN) }^{\mathrm{a}}\end{array}$ \\
\hline $\mathbf{t}_{\mathbf{0}}$ & $r \quad(p)$ & $r \quad(p)$ & $r \quad(p)$ \\
\hline $\begin{array}{l}\text { PR } \\
\text { ELAN }\end{array}$ & $.802(.000)$ & $.255(.061)$ & - \\
\hline \multicolumn{4}{|l|}{$\mathbf{t}_{1}$} \\
\hline WS & $.270(.101)$ & $-.143(.405)$ & $.382(.009)$ \\
\hline $\begin{array}{l}\text { VS } \\
\text { 3-jähr. }\end{array}$ & $-.125(.544)$ & $-.313(.119)$ & $.209(.152)$ \\
\hline $\begin{array}{l}\text { MR } \\
\text { 3-jähr. }\end{array}$ & $-.038(.854)$ & $.037(.856)$ & $.090(.331)$ \\
\hline $\begin{array}{l}\text { VS } \\
\text { 4-jähr. }\end{array}$ & $.505(.004)$ & $.666(.000)$ & $.463(.004)$ \\
\hline $\begin{array}{l}\text { MR } \\
\text { 4-jähr. }\end{array}$ & $.548(.001)$ & $.567(.001)$ & $.658(.000)$ \\
\hline $\mathrm{SG}$ & $.448(.012)$ & $.296(.119)$ & $.424(.009)$ \\
\hline \multicolumn{4}{|l|}{$\mathbf{t}_{2}$} \\
\hline WS & $.377(.005)$ & $.151(.285)$ & $.515(.000)$ \\
\hline $\mathrm{VS}$ & $.283(.038)$ & $.057(.690)$ & $.364(.003)$ \\
\hline MR & $.282(.039)$ & $.140(.321)$ & $.376(.003)$ \\
\hline $\mathrm{SG}$ & $.321(.031)$ & $.085(.590)$ & $.432(.002)$ \\
\hline \multicolumn{4}{|l|}{$\mathbf{t}_{\mathbf{3}}$} \\
\hline WS & $.179(.204)$ & $.111(.442)$ & $.276(.024)$ \\
\hline HSET VS & $.183(.191)$ & $.150(.294)$ & $.215(.061)$ \\
\hline PS & $.066(.638)$ & $.008(.957)$ & $.300(.015)$ \\
\hline IS & $.269(.051)$ & $.215(.129)$ & $.384(.002)$ \\
\hline
\end{tabular}

Anmerkungen. $\mathrm{t}_{0}=$ Messzeitpunkt 2001, $\mathrm{t}_{1,2,3}=$ Messzeitpunkt 1 bis 3, PR ELAN = Prozentrang im ELAN, WS = Wortschatz, VS = rezeptive grammatische Fähigkeiten gemessen mit dem Untertest „Verstehen von Sätzen“ (VS) aus dem SETK 3-5, MR = expressive grammatische Fähigkeiten gemessen mit dem Untertest „Morphologische Regelbildung“ (MR) aus dem SETK 3-5, SG = Satzgedächtnis, HSET VS = rezeptive grammatische Fähigkeiten gemessen mit dem HSET VS, PS = expressive grammatische Fähigkeiten gemessen mit dem Untertest „Plural-Singular-Bildung“ (PS) aus dem HSET, IS = Satzgedächtnis gemessen mit dem Untertest „Imitation grammatischer Strukturformen“ (IS) aus dem HSET, $r=$ Pearson Produkt Moment Korrelationen, $p=$ Fehlerwahrscheinlichkeit

a einseitige Testung 
Für die Summe der Funktionswörter im ELAN ergaben sich signifikante positive Korrelationen zu 8 der 14 Sprachoutcomevariablen in einer Höhe von .27 - .55. Keine signifikanten Korrelationen ergaben sich mit Ausnahme des Satzgedächtnisses für die Sprachoutcomevariablen zu $\mathrm{t}_{3}$ sowie für den Untertest WS zu t $\mathrm{t}_{1}$. Für die Untertests VS und MR bezogen auf die dreijährigen Kinder $z t_{1}$ ergaben sich nicht signifikante Korrelationen negativer Ausprägung. Tendenziell höhere Korrelationen im Vergleich zum Prozentrang im ELAN wurden für die Untertests VS (vierjährige Kinder) und SG zu t $\mathrm{t}_{1}$ errechnet.

Der Quotient aus der Summe der Funktionswörter im ELAN und Summe der Inhaltswörter im ELAN (QFI) korrelierte lediglich mit zwei Sprachoutcomevariablen signifikant: mit dem Untertest VS (vierjähriger Kinder; $r=.67 ; p=.000$ ) und dem Untertest MR (vierjährige Kinder; $r=.57 ; p=.001$ ) $\mathrm{zu} \mathrm{t}_{1}$. Tendenziell höhere Korrelationen als für den Gesamtwortschatzumfang bestanden zum Untertest VS für die vierjährigen Kinder zu $\mathrm{t}_{1}$.

Die Korrelationen der Summe an Funktionswörtern aus dem ELAN mit dem Prozentrang im ELAN belief sich auf .80 $(p=.000)$. Der QFI korrelierte in einer Höhe von $.255(p=.06)$ mit dem Prozentrang im ELAN.

Für die Summe aller Funktionswörter im ELAN wurden als echte Teilmenge des Gesamtwortschatzes analog zum Vorgehen in 7.1.2 alle Regressionsanalysen mit Forced Entry durchgeführt. Dieses Vorgehen war für den QFI nicht notwendig. Hier wurden hierarchische Regressionsanalysen mit dem Prozentrang im ELAN im ersten Block und dem QFI im zweiten Block gerechnet. Die Kriteriumsvariablen sind die beschriebenen Sprachoutcomevariablen aus Kapitel 7.1.2.

Die Summe aller Funktionswörter im ELAN erbrachte keine signifikante Varianzaufklärung für die Residuen aus den einfachen Regressionsanalysen mit dem Prozentrang im ELAN als Prädiktorvariable und den Sprachoutcomevariablen als Kriteriumsvariablen.

Für den QFI konnte ein signifikanter zusätzlicher Varianzaufklärungsbeitrag für den Untertest VS (vierjährige Kinder) $\mathrm{zu} \mathrm{t}_{1}$ über den Prozentrang im ELAN hinaus berechnet werden $\left(\Delta R^{2}=.26 ; F=11.92 ; p=.000\right)$.

Die Prüfung der bestehenden Korrelationsdifferenzen ergab hingegen auch für die Korrelation von QFI mit dem Untertest VS (vierjährige Kinder) zu $\mathrm{t}_{1}$ im Vergleich zur entsprechenden Korrelation des Prozentranges im ELAN keine signifikanten Ergebnisse. 


\subsubsection{Diskussion}

Für die Summe der Funktionswörter im ELAN konnten in Anbetracht der kleinen Itemmenge von 23 Wörtern erstaunlich hohe signifikante Korrelation zu allen Sprachoutcomevariablen zu $\mathrm{t}_{2}$ sowie zu einigen Sprachoutcomevariablen zu $\mathrm{t}_{1}$ und zum Satzgedächtnis zu $\mathrm{t}_{3}(r=.27-.55)$ errechnet werden. Für den Wortschatz $\mathrm{zu} \mathrm{t}_{1}$ wurde die Signifikanz knapp verfehlt, was aufgrund der kleinen Teilstichprobe von 38 Kindern und dem wenig altersgerechten Erhebungsverfahren (AWST 3-6) für diesen Zeitpunkt nicht überbewertet werden sollte. Zu den grammatischen Leistungen der Teilstichprobe der dreijährigen Kinder $\mathrm{zu} \mathrm{t}_{1}$ bestanden negative Korrelationen nicht signifikanter Ausprägung. Auch dieses Ergebnis muss relativiert werden, da es inhaltlich nicht zu erklären ist. Denn warum sollte eine Vielzahl von grammatisch bedeutsamen Wörtern mit schlechteren grammatischen Leistungen in Zusammenhang stehen? Zudem beziehen sich die Ergebnisse lediglich auf eine Teilstichprobe von 26 Kindern.

Aufgrund der hohen Korrelation von der Summe aller Funktionswörter im ELAN mit dem Prozentrang im ELAN ( $r=.80)$ wundert es nicht, dass keine zusätzliche Varianz für späteres Sprachoutcome über den gesamten Wortschatzumfang hinaus aufgeklärt werden konnte und auch keine höheren Zusammenhänge $\mathrm{zu}$ späterem Spachoutcome als für den Gesamtwortschatz bestanden. Dieser hohe Zusammenhang von Wortschatzgröße und Anzahl an Funktionswörtern ist in der Literatur belegt (Bates et al., 1994) und spricht auch für die Repräsentativität der vorliegenden Daten.

Für den QFI sieht das Ergebnismuster anders aus. Das Verhältnis von Funktions- zu Inhaltswörtern kann die Vorhersage der rezeptiven grammatischen Leistungen $\mathrm{zu} \mathrm{t}_{1}$ (für die Stichprobe der vierjährigen Kinder) aus dem Gesamtwortschatz substanziell verbessern. So scheint der QFI Informationen zu repräsentieren, die nicht im Gesamtwortschatzumfang enthalten sind. Dieses lässt sich auch an den geringen Korrelationen von QFI und Prozentrang im ELAN in der Höhe von .25 ablesen. Insgesamt bestehen für das Verhältnis von Funktionsund Inhaltswörtern jedoch nur zu zwei Sprachoutcomevariablen (VS und MR zu $\mathrm{t}_{1}$ der vierjährigen Kinder) signifikante Zusammenhänge und dieses Ergebnis sollte kritisch betrachtet werden, da es an einer Teilstichprobe von 31 Kindern erhoben wurde. Ungeklärt bleibt, warum der QFI gerade mir diesen zwei Sprachoutcomevariablen signifikant korreliert und warum für die rezeptiven grammatischen Leistungen zusätzliche bedeutsame Varianz aufgeklärt werden kann, nicht aber für die expressiven. Zur Vorhersage späterer sprachlicher Leistungen sollte der QFI nicht verwendet werden. Bedeutsame Verbesserungen der Vorhersage lieferte er nur in einem Fall. 
Funktionswörter im frühen kindlichen Wortschatz bilden sich erst verstärkt ab einer Wortschatzgröße von 200 Wörtern (Bates et al., 1994). In der hier untersuchten Stichprobe lag der durchschnittliche mit dem ELAN erhobene Wortschatzumfang bei 99.25 Wörtern (SD = 82.3). Dieser Tatbestand gibt zu bedenken, dass bei einer Stichprobe mit durchschnittlichem Wortschatzumfang von mindestens 200 Worten sowie geringerer Variabilität der Wortschatzgröße andere Ergebnisse in Bezug auf die Funktionswörter sowie das Verhältnis von Funktions- und Inhaltswörtern hinsichtlich späterem Sprachoutcome hätten resultieren können.

\subsection{Semantische Kategorien}

\subsubsection{Theoretischer Hintergrund}

Auf der theoretisch-semantischen Ebene können Objekte, deren Benennungen und ihre semantischen Informationen in Kategorien (z.B. Tiere und Fahrzeuge) und Domänen (z.B. belebt oder zählbar) eingeteilt werden (Wengenrath, 2006).

In der sprachpsychologischen Literatur überwiegt die Einteilung in Kategorien, wobei unterschiedliche Begrifflichkeiten für die Kategorien des Wortschatzes gebräuchlich sind. Diese werden unter anderem als semantische Felder, semantische Kategorien, Wortfelder und Bedeutungsfelder bezeichnet (z.B. Szagun \& Steinbrink, 2004). Der Begriff Wortfeld wurde von Trier $(1931,1973)$ etabliert und beschreibt eine Gruppe von sinnverwandten Wörtern einer Sprache, deren Bedeutungen sich gegenseitig begrenzen und lückenlos einen bestimmten begrifflichen Bereich abdecken. In der englischsprachigen Literatur überwiegt der Begriff „semantic categories“ (z.B. Berglund \& Eriksson, 2000). In Anlehnung an die englischsprachige Literatur wird in vorliegender Arbeit der Begriff semantische Kategorie verwendet.

Die semantische Entwicklung ist charakterisiert durch eine beständige Elaboration und Ausdifferenzierung der semantischen Kategorien. Der Bedeutungsgehalt erster Worte bezieht sich auf direkt erfahrbare Gegebenheiten aus der kindlichen Umwelt und der Einstieg in die Wortschatzentwicklung erfolgt über Basic-Level-Begriffe aus den typischen frühen semantischen Feldern wie Essen, Körper oder Tiere. Dann kommt es zu einer Erhöhung der Anzahl der semantischen Kategorien sowie der Ausdifferenzierung innerhalb einer semantischen Kategorie z.B. durch den Erwerb abstrakter Begriffe.

Die bewährten klinischen Parent Report Verfahren zur Erfassung des frühen Wortschatzes wie z.B. die CDI, LDS oder ELI strukturieren die Wortschatzlisten nach semantischen Kategorien (auch um den Abruf der Informationen für die ausfüllenden Eltern zu erleichtern). 
Semantische Kategorien in den Wortschatzchecklisten dieser Verfahren sind z.B. Körperteile, Spielzeuge und Tiere, also Kategorien, die aus Substantiven bestehen und ausschließlich Inhaltswörter beinhalten. Ebenso gibt es Kategorien, die nur aus Funktionswörtern bestehen wie z.B. Pronomen und Artikel. Keines der Verfahren erhebt jedoch die Anzahl semantischer Kategorien, die ein Kind mit Begrifflichkeiten gefüllt hat, als Maß für den aktuellen Stand der Wortschatzentwicklung.

Die Anzahl semantischer Kategorien, die ein Kind belegt hat, mag ein Indikator dafür sein, wie gut ein Kind in der Lage ist, sein Wissen hierarchisch konzeptuell zu strukturieren. Fraglich ist hierbei, ab wann eine semantische Kategorie als belegt gilt. Sollte schon bei einem einzigen Eintrag die semantische Kategorie als solche gewertet werden oder erst ab einer bestimmten Mindestbelegung von der Beherrschung dieser Kategorisierung ausgegangen werden? Klärende Hinweise $\mathrm{zu}$ dieser Frage konnten der aktuellen sprachpsychologischen Literatur nicht entnommen werden.

Da die Strukturierung von Wissen weiteren sprachlichen Wissenserwerb erleichtert (Weinert, 2004), wird geschlossen, dass ein Kind, welches in der Lage ist viele semantische Kategorien aufzubauen, über ein besonders gutes kognitives Potential für die weiteren Spracherwerbsaufgaben verfügt. Die kognitive Aufgabe der hierarchisch konzeptuellen Strukturierung sprachlichen Materials dürfte je nach semantischer Kategorie unterschiedlich anspruchsvoll sein. Semantische Kategorien werden in einer bestimmten Erwerbsreihenfolge erworben, da sie unterschiedliche Wortarten enthalten bzw. repräsentieren. Bestimmte Wortarten sind leichter zu erwerben als andere und Inhaltswörter sind leichter zu erwerben als Funktionswörter, da sie einen eigenen Bedeutungsgehalt haben (vgl. Kap. 7.1 und 7.2). Folglich wird gerade die Anzahl nicht substantivisch gefüllter semantischer Kategorien als guter Prädiktor für die spätere Sprachentwicklung angenommen.

Studien zur prädiktiven Validität der Anzahl semantischer Kategorien für spätere Sprachleistungen sowie Studien zum Zusammenhang der Anzahl semantischer Kategorien zu späteren Sprachleistungen bis ins Grundschulalter konnten der aktuellen Forschungsliteratur nicht entnommen werden.

Fraglich ist, ob die Anzahl semantischer Kategorien zusätzlich zum Wortschatzumfang die Vorhersage späterer Sprachleistungen verbessern kann und ob höhere Zusammenhänge der Anzahl semantischer Kategorien zu späterem Sprachoutcome im Vergleich zum expressiven Wortschatzumfang bestehen. 


\subsubsection{Ergebnisse}

Als Sprachoutcomevariablen fungierten die Rohsummen der gleichen Untertests wie in 7.1.2. beschrieben. Für die Operationalisierung der Kategorisierung des kindlichen Wortschatzes wurde zum einen die Summe aller belegten semantischen Kategorien im ELAN (Max = 17; „Semkatanz") verwendet und zum anderen die Summe aller nichtsubstantivischen semantischen Kategorien im ELAN (Fragewörter, Zahlen/Mengen, Sonstige, Hilfsverben, Fürwörter/Artikel, Qualitäten und Tätigkeiten; Max = 7; „Semnisub“) in die Berechnungen eingegeben. Hierbei galt eine semantische Kategorie als belegt, wenn zumindest ein Item der Kategorie im ELAN von den Eltern angekreuzt worden war. Darüber hinaus wurden als Variablen die Anzahl aller semantischen Kategorien sowie die Anzahl aller nichtsubstantivischen Kategorien im ELAN bei der notwendigen Voraussetzung einer Minimalbelegung von 25\% generiert (,Semanz25“ und „Semnisub25“).

Analog zum Vorgehen in Kapitel 7.1.2 wurden im ersten Schritt die Korrelationen der vier Variablen mit den Sprachoutcomevariablen berechnet und auf Signifikanz geprüft $(\alpha=.10)$. Tabelle 16 stellt die Ergebnisse dar und veranschaulicht zusätzlich die Korrelationen des Prozentranges im ELAN mit den Sprachoutcomevariablen. Signifikante Korrelationen sind bezüglich des Gesamtwortschatzes rot und für die semantischen Kategorien gelb hinterlegt. 
Tabelle 16

Zusammenhänge von Anzahl der semantischen Kategorien und Gesamtwortschatzumfang mit Sprachoutcome von $t_{0}$ bis $t_{3}$

\begin{tabular}{|c|c|c|c|c|c|}
\hline & $\begin{array}{l}\text { Anzahl aller } \\
\text { semantischen } \\
\text { Kategorien }\end{array}$ & $\begin{array}{l}\text { Anzahl aller } \\
\text { semantischen } \\
\text { Kategorien } \\
\text { (Minimal-- } \\
\text { belegung } \\
25 \% \text { ) }\end{array}$ & $\begin{array}{l}\text { Anzahl nicht } \\
\text { substanti- } \\
\text { vischer } \\
\text { semantischer } \\
\text { Kategorien }\end{array}$ & $\begin{array}{l}\text { Anzahl nicht } \\
\text { substanti- } \\
\text { vischer } \\
\text { semantischer } \\
\text { Kategorien } \\
\text { (Minimal- } \\
\text { belegung } \\
25 \% \text { ) }\end{array}$ & $\begin{array}{l}\text { Gesam- } \\
\text { twortschatz } \\
(\text { PR ELAN) }\end{array}$ \\
\hline $\mathbf{t}_{\mathbf{0}}$ & $\begin{array}{ll}r & (p) \\
\end{array}$ & $\begin{array}{ll}r & (p) \\
\end{array}$ & $\begin{array}{ll}r & (p) \\
\end{array}$ & $\begin{array}{ll}r & (p) \\
\end{array}$ & $\begin{array}{ll}r & (p) \\
\end{array}$ \\
\hline $\begin{array}{l}\text { PR } \\
\text { ELAN }\end{array}$ & $.750(.000)$ & $.825(.000)$ & $.771(.000)$ & $.787(.000)$ & - \\
\hline \multicolumn{6}{|l|}{$\mathbf{t}_{1}$} \\
\hline WS & $.346(.033)$ & $.317(.053)$ & $.337(.041)$ & $.307(.064)$ & $.382(.009)$ \\
\hline $\begin{array}{l}\text { VS } \\
\text { 3-jähr. }\end{array}$ & $-.009(.966)$ & $.032(.876)$ & $.041(.843)$ & $.071(.729)$ & $.209(.152)$ \\
\hline $\begin{array}{l}\text { MR } \\
\text { 3-jähr. }\end{array}$ & $-.277(.171)$ & $-.195(.340)$ & $-.292(.148)$ & $-.042(.838)$ & $.090(.331)$ \\
\hline $\begin{array}{l}\text { VS } \\
\text { 4-jähr. }\end{array}$ & $.503(.004)$ & $.444(.012)$ & $.619(.000)$ & $.461(.010)$ & $.463(.004)$ \\
\hline $\begin{array}{l}\text { MR } \\
\text { 4-jähr }\end{array}$ & $.595(.000)$ & $.602(.000)$ & $.675(.000)$ & $.575(.001)$ & $.658(.000)$ \\
\hline $\mathrm{SG}$ & $.353(.052)$ & $.441(.013)$ & $.430(.018)$ & $.450(.012)$ & $.424(.009)$ \\
\hline \multicolumn{6}{|l|}{$t_{2}$} \\
\hline WS & $.355(.008)$ & $.451(.001)$ & $.394(.004)$ & $.457(.001)$ & $.515(.000)$ \\
\hline $\mathrm{VS}$ & $.396(.003)$ & $.379(.005)$ & $.427(.001)$ & $.341(.013)$ & $.364(.003)$ \\
\hline $\mathrm{MR}$ & $.198(.151)$ & $.264(.054)$ & $.292(.034)$ & $.294(.032)$ & $.376(.003)$ \\
\hline $\mathrm{SG}$ & $.125(.414)$ & $.258(.087)$ & $.272(.074)$ & $.272(.074)$ & $.432(.002)$ \\
\hline \multicolumn{6}{|l|}{$\mathbf{t}_{3}$} \\
\hline $\mathrm{WS}$ & $.128(.366)$ & $.219(.120)$ & $.189(.185)$ & $.213(.134)$ & $.276(.024)$ \\
\hline HSET VS & $.076(.591)$ & $.161(.250)$ & $.147(.298)$ & $.183(.193)$ & $.215(.061)$ \\
\hline PS & $.058(.682)$ & $.171(.221)$ & $.076(.592)$ & $.137(.333)$ & $.300(.015)$ \\
\hline IS & $.084(.551)$ & $.213(.126)$ & $.194(.168)$ & $.230(.102)$ & $.384(.002)$ \\
\hline
\end{tabular}

Anmerkungen $. \mathrm{t}_{0}=$ Messzeitpunkt 2001, $\mathrm{t}_{1,2,3}=$ Messzeitpunkt 1 bis 3, PR ELAN = Prozentrang im ELAN, WS = Wortschatz, VS = rezeptive grammatische Fähigkeiten gemessen mit dem Untertest „Verstehen von Sätzen“ (VS) aus dem SETK 3-5, MR = expressive grammatische Fähigkeiten gemessen mit dem Untertest „Morphologische Regelbildung“ (MR) aus dem SETK 3-5, SG = Satzgedächtnis, HSET VS = rezeptive grammatische Fähigkeiten gemessen mit dem HSET VS, PS = expressive grammatische Fähigkeiten gemessen mit dem Untertest „Plural-Singular-Bildung“(PS) aus dem HSET, IS = Satzgedächtnis gemessen mit dem Untertest „Imitation grammatischer Strukturformen“(IS) aus dem HSET, $r=$ Pearson Produkt Moment Korrelationen, $p=$ Fehlerwahrscheinlichkeit a einseitige Testung 
Es ergaben sich keine signifikanten Korrelationen für die Variablen Semkatanz, Semnisub, Semanz25 und Semnisub25 mit den Sprachoutcomevariablen zu t t $_{3}$

Mit allen Sprachoutcomevariablen zu $t_{2}$ korrelierten die Variablen Semanz25, Semnisub und Semnisub25 in signifikantem Ausmaß von .26 - .46. Sie Summe aller belegten semantischen Kategorien (Semkatanz) korrelierte nur mit den Untertests WS und VS zu $t_{2}$ signifikant ( $r=.35$ und .40). Mit den Sprachoutcomevariablen zu t $t_{1}$ ergaben sich analog zum Prozentrang im ELAN keine signifikanten Korrelationen für die Untertests VS und MR für die dreijährigen Kinder. Für die Untertests WS, SG sowie MR und VS (bezogen auf die vierjährigen Kinder) ergaben sich signifikanten Korrelationen in der Höhe von .32 - .67.

Tendenziell höhere Korrelationen mit den Sprachoutcomevariablen ließen sich im Vergleich zum Prozentrang im ELAN für die Variablen Semkatanz (VS Vierjährige zu $t_{1}$ und VS zu t $t_{2}$ ), Semanz25 (SG zu t $t_{1}$ und VS zu t $t_{2}$ ), Semnisub (VS und MR Vierjährige sowie SG $\mathrm{zu} \mathrm{t}_{1}$ und VS zu t ${ }_{2}$ ) sowie Semnisub25 (SG zu t $\mathrm{t}_{1}$ ) berechnen.

Alle vier Variablen korrelierten signifikant positiv und mit hoher Effektstärke mit dem Prozentrang im ELAN $(r=.75-.82 ; p=.000)$.

Für die vier Variablen wurden hierarchische Regressionsanalysen mit der Prädiktorvariable Prozentrang im ELAN im ersten Block und den Variablen selbst (Semkatanz, Semanz25, Semnisub und Semnisub25) als Prädiktorvariable im zweiten Block durchgeführt. Kriteriumsvariablen waren die bekannten Sprachoutcomevariablen (vgl. 7.1.2).

Keine der Regressionsanalysen ergab eine signifikante zusätzliche Varianzaufklärung über den Prozentrang im ELAN hinaus für die Variablen Semkatanz, Semanz25, Semnisub und Semnisub25.

Für die tendenziell höheren Korrelationen der Variablen Semkatanz, Semanz25, Semnisub und Semnisub25 mit späterem Sprachoutcome im Vergleich zum Prozentrang im ELAN wurden die entsprechenden Korrelationsdifferenzen auf Signifikanz geprüft. Es lagen keine signifikanten Korrelationsdifferenzen vor.

\subsubsection{Diskussion}

Die Anzahl der belegten semantischen Kategorien korrelierte signifikant und in hohem Ausmaß mit dem Prozentrang im ELAN ( $r=$.75-.82), was für einen hohen Zusammenhang von Gesamtwortschatz und Aufteilung desselbigen auf bestimmte semantische Kategorien spricht. Gleichzeitig macht dieses Ergebnis deutlich, dass die Anzahl semantischer Kategorien 
ähnliche Informationen trägt wie der Gesamtwortschatz. Dieses zeigt sich auch in den Korrelationen zu späterem Sprachoutcome. Für die Variablen Semkatanz, Semanz25, Semnisub und Semnisub25 bestehen ähnlich hohe Korrelationen zu den Sprachoutcomevariablen wie für den Prozentrang im ELAN. Dass im Unterschied zum Prozentrang im ELAN keine signifikanten Korrelationen zu den Sprachoutcomevariablen zum Zeitpunkt $t_{3}$ vorliegen, mag durch die geringere Reliabilität der Messung (vgl. kleine Itemmengen von Max = 7 bzw. Max = 17) bedingt sein, die sich gerade über die große Zeitspanne von 6 Jahren auswirkt. Die Variable mit den meisten tendenziell höheren Korrelationen zu späterem Sprachoutcome im Vergleich zum Prozentrang im ELAN ist die Anzahl nicht substantivischer Kategorien, was die Bedeutung dieser Kategorien für die weitere Sprachentwicklung unterstreicht. Tendenziell scheint das Ausmaß der Belegung einer semantischen Kategorie (1 Eintrag vs. 25\% Belegung) für die Höhe der bestehenden Zusammenhänge zu späteren Sprachoutcome keine bedeutsamen Unterschiede für die Anzahl aller semantischer Kategorien bei einfachem Belegungskriterium nur sieben signifikante Korrelationen zu späterem Sprachoutcome, bei 25\% Belegung hingegen neun. Warum dieses so ist, lässt sich vermuten. Denn eine $25 \%$ Belegung spricht für einen größeren Wortschatz insgesamt, der wiederum zu höheren Korrelationen mit dem Sprachoutcome führt.

Es wundert nicht, dass bei so hohem Zusammenhang von der Anzahl semantischer Kategorien und Wortschatzumfang keine substanziell höheren Zusammenhänge für die Anzahl semantischer Kategorien mit späterem Sprachoutcome im Vergleich zum Wortschatzumfang bestehen und auch keine Verbesserung der Vorhersage späterer Sprachleistungen aus dem gesamten Wortschatzumfang erzielt wurde.

Insgesamt bleibt fraglich, ob die Anzahl belegter semantischer Kategorien im frühen kindlichen Wortschatz tatsächlich eine (kognitive) Kategorisierungs- oder Strukturierungsleistung des Kindes repräsentiert. Der Tatbestand, dass ein Kind z.B. über die Worte „Auto“ und „Hund“ verfügt, garantiert ja nicht, dass es die entsprechenden Objekte in einer Zuordnungsaufgabe den semantischen Kategorien (Fahrzeuge und Tiere) bzw. anderen Objekten aus diesen semantischen Kategorien (Trecker und Esel) korrekt zuordnen kann. Die vermutete Kategorisierungsleistung liegt wahrscheinlich stärker auf der Seite des Testentwicklers, der den frühen kindlichen Wortschatz in semantischen Kategorien sortiert präsentiert und abfragt. Die prozentuale Belegung der semantischen Kategorien bezüglich der substantivischen Kategorien könnte die Interessen des Kindes und dessen sprachliche Lernumwelt widerspiegeln, wohingegen die Anzahl aller belegten Kategorien einfach das „Abstract“ des gesamten Wortschatzumfanges mit geringerer Messgüte zu sein scheint. 
Die Anzahl belegter nichtsubstantivischer Kategorien führt $\mathrm{zu} \mathrm{t}_{1} \mathrm{zu}$ tendenziell höheren Korrelationen mit allen grammatischen Sprachmaßen und scheint als „Abstract“ der grammatischen Fähigkeiten zumindest für Vorhersagen späteren Sprachoutcomes, die einen Zeitraum von bis zu 26 Monaten betreffen, als leicht zu ermittelnder Prädiktor neben dem Gesamtwortschatzumfang Berechtigung zu haben und weitere Forschungsbemühungen z.B. an größeren Stichproben und auf Basis der critical mass hypothesis zu lohnen.

Die qualitativen Analysen des frühen Wortschatzes erbrachten in der Zusammenschau und nach kritischer Bewertung der Daten keine bedeutsamen Verbesserungen der Vorhersage späteren Sprachoutcomes aus dem expressiven Wortschatzumfang. Es ließen sich zudem keine substanziell höheren Zusammenhänge zu späterem Sprachoutcome im Vergleich zur bewährten quantitativen Analyse finden.

Insgesamt haben die qualitativen Analysen des frühen Wortschatzes jedoch zum einen unterstrichen, dass der expressive Wortschatzumfang ein guter Repräsentant für die Informationen ist, die in allen Teilmengen des Wortschatzes enthalten sind. Dieses muss nicht bezogen auf jedes Erhebungsverfahren zur Erfassung des frühen Wortschatzes Gültigkeit haben und mag an der besonders empirienahen Entwicklung des ELANs aus den Tagebuchaufzeichnungen von über 40 Eltern mit begründet sein. Die qualitativen Analysen haben neue Forschungsfragen aufgeworfen und Variablen hervorgebracht, wie z.B. den QFI und die Anzahl belegter nichtsubstantivischer Kategorien, die weitere Forschungsbemühungen lohnenswert erscheinen lassen. 


\section{Ausblick}

Grundsätzlich gilt für alle bearbeiteten Forschungsfragen in dieser Arbeit der Wunsch nach einer Replizierung der Ergebnisse an einer größeren Stichprobe $\mathrm{zu}$ mehr Untersuchungszeitpunkten, die in kürzerem Zeitabstand zueinander liegen. Dabei sollte die Versuchplanung auch auf die verwendeten Erhebungsverfahren so gut wie möglich abgestimmt sein, um z.B. eine Teilung der Stichproben zu bestimmten Messzeitpunkten zu vermeiden (vgl. $t_{1}$ in dieser Untersuchung). Gleichzeitig muss eingeschränkt werden, dass insbesondere bei der Umsetzung aktueller Forschungsfragen im längsschnittlichen Untersuchungsdesign den theoretisch fundierten Forderungen und Ansprüchen an die Versuchsplanung Einschränkungen in der praktischen Umsetzbarkeit gegenüberstehen. So müssen z.B. bei der Planung der Untersuchungsintervalle auch Testwiederholungseffekte einberechnet werden und schon bei einer Stichprobengröße von 57 Kindern, wie in der vorliegenden Untersuchung, stellen Untersuchungsplanung, Datenerhebung und -auswertung, Rückmeldung an die Eltern und Aufrechterhaltung der Motivation zur Teilnahme über eine Zeitspanne von 6 Jahren große Ansprüche an die verfügbaren Ressourcen auf Seiten des Untersuchers.

Eine Reihe von Überlegungen für weitere Forschungsansätze sowie methodische Optimierungspotenziale ergeben sich darüber hinaus aus der Diskussion der Ergebnisse bezogen auf die unterschiedlichen Forschungsfragen in dieser Arbeit.

\subsection{Entwicklungsstabilitäten}

In weiteren Analysen der Daten dieser Arbeit könnte der Frage nachgegangen werden, ob es bestimmte Sprachkomponenten gibt, die von bedeutsam höherer Stabilität sind als andere. Hierzu müssten pro Zeitfenster die Korrelationsdifferenzen für die unterschiedlichen Sprachaspekte auf Signifikanz geprüft werden. Für einzelne Zeitfenster könnten signifikante Differenzen vorliegen (z.B. $\mathrm{t}_{1} \mathrm{zu} \mathrm{t}_{2} \mathrm{r}=.87$ [Wortschatz)] vs. $\mathrm{r}=.51$ [rezeptive grammatische Fähigkeiten]); durchgängig über alle Zeitintervalle ist jedoch angesichts der berichteten Korrelationen für die unterschiedlichen sprachlichen Bereiche kaum zu erwarten, dass eine Komponente sich als besonders stabil im Vergleich zu den anderen abhebt. Entsprechende Untersuchungen sollten an größeren Stichproben, bei identisch häufigem Wechsel in den Erhebungsverfahren und vergleichbar guten Operationalisierungen der sprachlichen Untersuchungsaspekte durchgeführt werden.

Im Rahmen dieser Arbeit wurden die normativen oder Positionsstabilitäten für gleiche Leistungsbereiche untersucht. Dieses lässt keine Aussagen für die heterotypen Stabilitäten zu. 
$\mathrm{Zu}$ erwarten ist, dass diese niedriger sind als die homotypen Stabilitäten. Hinsichtlich dieses Aspektes könnte die Datenbasis eine erweiterte Auswertung erfahren. Die heterotypen Stabilitäten (Korrelationen zwischen dem Abschneiden in unterschiedlichen Sprachaspekten) bildeten die Grundlage für die Auswertungen in Kapitel 4, in dem es um die Vorhersage späterer Sprachleistungen aus dem frühen Wortschatz ging, wurden aber nicht bezüglich des Aspektes Stabilität bewertet.

Für die grammatischen Fähigkeiten (rezeptive und expressive) sowie für das Satzgedächtnis wären frühere Operationalisierungen ( $\left.\mathrm{zu} \mathrm{t}_{0}\right)$ anzustreben, damit, wie für den Wortschatz, auch für diese Sprachaspekte Aussagen zur Stabilität schon im frühen Kindesalter gemacht werden können. Eine mögliche Variable zur Operationalisierung der expressiven grammatischen Fähigkeiten im frühen Kindesalter wäre die durchschnittliche Äußerungslänge (MLU). Ihr müsste jedoch eine äquivalente Variable für die späteren Messzeitpunkte gegenüberstehen (z.B. die in Spontansprachanalysen ermittelte MLU).

Die dokumentierten Ergebnisse zur Stabilität von Sprache erlauben keine Rückschlüsse auf die verursachenden Bedingungen. So könnten sowohl stabile endogene Faktoren als auch die Stabilität der Umweltbedingungen ursächlich für die Ergebnisse sein. Weiterführende Untersuchungen, die parallel die Stabilität der Umweltbedingungen erfassen, könnten hier Erkenntnisgewinn bringen und zur bestehenden allgemeinen Forschungslücke für den Untersuchungsgegenstand „Stabilität von Umweltbedingungen“ (Spiel et al., in Druck) beitragen.

\subsection{Prädiktive Validität des frühen Wortschatzes}

Für die Güte sowie die Vergleichbarkeit der Vorhersagen über die unterschiedlichen Zeitintervalle sollte in weiteren Forschungsprojekten darauf geachtet werden, möglichst wenig Wechsel in den verwendeten Untersuchungsverfahren und der Art der Operationalisierung eines Konstruktes vorzunehmen. Bei einer so großen Zeitspanne der Untersuchung von früher Kindheit bis ins Grundschulalter ist jedoch die Verwendung unterschiedlicher Untersuchungsverfahren gar nicht zu vermeiden. Entscheidender für die Beantwortung der Forschungsfragen ist die Güte der verwendeten Verfahren. Begründete Zweifel an der Güte der Verfahren ergaben sich lediglich für den AWST 3-6 zur Erfassung des Wortschatzes für junge Kinder (unter 5 Jahren). Hier sollte in folgenden Studien der AWST-R eingesetzt werden.

Insgesamt wäre die Replizierung der Ergebnisse an einer größeren Stichprobe für die Untersuchung der prädiktiven Validität besonders wünschenswert, denn Bornstein et al. 
(2004) dokumentierten unterschiedliche Ergebnisse zur prädiktiven Validität des frühen Wortschatzes in Abhängigkeit von der Stichprobengröße.

Bei größerer Stichprobe wäre es auch möglich, das Erhebungsalter für den frühen Wortschatz systematisch zu variieren und Altersgruppen von Kindern im längsschnittlichen Design zu untersuchen. Bei identischen Untersuchungsverfahren und Erhebungsintervallen könnte man Aussagen zur Validität des Erhebungsalters für den frühen Wortschatz machen. Als mögliche Gruppierungsvariable würde sich der Wortschatzspurt anbieten (vor, während und nach dem Wortschatzspurt). Wollte man die critical mass hypothesis von Marchman und Bates (1994) im Hinblick auf die Güte von Vorhersagen späteren Sprachoutcomes prüfen, könnte man die Untersuchungsstichprobe darüber hinaus nach kritischem Wortschatzumfang gruppieren und dieses in die Vorhersage späterer Sprachleistungen einbeziehen. Dieses war aufgrund der relativ kleinen Stichprobe in dieser Studie nicht möglich. Es ist aber durchaus logisch nachvollziehbar, dass der frühe expressive Wortschatz ab einer bestimmten „Ausgereiftheit“" bessere Vorhersagen zulässt, denn abhängig von der Wortschatzgröße ändert sich auch die Wortschatzkomposition und Funktionswörter werden z.B. erst ab einer Wortschatzgröße von 200 verstärkt erworben (vgl. Kap. 7) und gelten als besonders gute Indikatoren für die grammatische Entwicklung eines Kindes.

Im Rahmen dieser Doktorarbeit wurden 53 Kinder zu 4 Messzeitpunkten vom frühen Kindesalter ( $M=22$ Monate) bis 7;10 Jahren untersucht. Der Beginn der Untersuchungen zu einem früheren Zeitpunkt würde weitere Einblicke in noch frühere Spracherwerbsprozesse ermöglichen. Die Erhebung rezeptiver sprachlicher Fähigkeiten und anderer möglicher Vorläuferfertigkeiten für die expressive Sprache (z.B. die Fähigkeit zur Speicherung und Wiedererkennung von Lautsequenzen unter unterschiedlichen Inputbedingungen; vgl. Höhle, 2004) könnten noch frühere und ggf. bessere Vorhersagen für späteres Sprachoutcome ergeben.

Auf jeden Fall wäre die Erhöhung der Anzahl der Messzeitpunkte wünschenswert, um Aussagen zur prädiktiven Validität für unterschiedliche Zeitspannen machen zu können. Besonders relevant ist dieses mit Hinblick auf die Sprachstandserhebungen vor der Einschulung. Was ist ein optimaler Zeitpunkt zur Erhebung der schulrelevanten sprachlichen Fähigkeiten und bietet einerseits eine hohe prognostische Validität für die Sprachleistungen im Schulalter und andererseits die Möglichkeit in der verbleibenden Zeit bei ungenügenden sprachlichen Leistungen noch Interventionsmaßnahmen einleiten zu können?

Weitergehende Analysen könnten die Güte der Vorhersagen aus dem frühen expressiven Wortschatz z.B. in Abhängigkeit vom Bildungsstand der Eltern, Geschlecht oder 
Geschwisterposition prüfen. Diesbezüglich liegen in der Literatur divergente Ergebnisse vor (vgl. Reese \& Read, 2000).

Bessere Vorhersagen späteren Sprachoutcomes könnte die Erhebung des Entwicklungstempos vom frühen Wortschatz (Wortschatzzuwachs) zwischen zwei Messzeitpunkten innerhalb eines besonders aussagekräftigen Zeitintervalls liefern. So charakterisieren Thal, Bates, Zappia \& Oroz (1996) sowie Berglund \& Eriksson (2000) den Wortschatzzuwachs als guten Prädiktor für die frühe Syntax und weitere grammatische Fähigkeiten. Hier wäre zu bestimmen, welches Erhebungsintervall in welchem Lebensalter der Kinder besonders gute Vorhersagen ermöglicht. Generell sollte die einmalige Erhebung von Leistungsmerkmalen im Rahmen von Entwicklungsvorhersagen überdacht werden. Die Vorhersage von Lese-Rechtschreibschwierigkeiten mit dem Bielefelder Screening zur Früherkennung von Lese-Rechtschreibschwäche (Jansen, Mannhaupt, Marx \& Skowronek, 2002) zum Beispiel führt nach jüngsten Untersuchungen erst bei wiederholter Durchführung (10 Monate und 4 Monate vor der Einschulung) zu befriedigenden Vorhersagen (P. Marx, persönliche Mitteilung, 9.1.2007).

Die theoriegeleitete Spracherwerbsforschung könnte zudem die Vorhersagen erfahrener Kliniker (z.B. KinderärztInnen, LogopädInnen und SprachheilpädagogInnen) auf ihre prognostische Validität prüfen und nach den Kriterien und Entscheidungsalgorithmen (Gewichtung und Kombination der Informationen) forschen, die $\mathrm{zu}$ besonders guten Vorhersagen späteren Sprachoutcomes führen. Wissenschaftlich evaluierte effektive Interventionsprogramme für Late Talker wie z.B. das Heidelberger Elterntraining zur frühen Sprachförderung (Buschmann et al., 2007) mögen zudem Rückschlüsse auf Merkmale oder Bedingungsfaktoren ermöglichen, die nicht nur für die Vorhersage in Bezug auf die gestörte Sprachentwicklung entscheidend sind und das Augenmerk auf entscheidende noch ungelöste Fragen der Sprachentwicklung lenken (z.B. Welche Merkmale der sozialen Sprachumwelt sind notwendig für einen erfolgreichen Spracherwerb?).

\subsection{Frühe grammatische Fähigkeiten}

Nicht geprüft wurde die psychologische Hypothese, dass frühe grammatische Fähigkeiten zu keiner bedeutsamen Verbesserung der Vorhersagen für späteres Sprachoutcome aus dem frühen Wortschatz führen, in Abhängigkeit vom Alter. Der Wortschatz wächst im frühen Kindesalter (1;6 bis 2;6 Jahren) schneller an als die MLU und diese verfügt über die höchste interindividuelle Variabilität im Alter von 2;4 bis 2;6 Jahren (Szagun \& Steinbrink, 2004). Eine Untersuchung an einer größeren Stichprobe, welche die Prüfung der Hypothese an 
unterschiedlichen Altersgruppen auch in höherem Alter als 26 Monaten erlaubt, könnte zu anderen Ergebnissen bzw. neuen Erkenntnissen führen. So könnte die MLU im Alter von 3;0 bis 3;6 Jahren substanzielle zusätzliche Varianz bei der Vorhersage späterer grammatischer Fertigkeiten aus dem frühen Wortschatz vorhersagen, da sie dann der Wortschatzentwicklung nicht mehr „hinterherhinkt“ und damit über einen größeren Anteil wortschatzunabhängiger sprachentwicklungsrelevanter Informationen verfügt.

Fraglich ist, ob die Eltern mit der Angabe von drei typischen Äußerungen ihres Kindes im ELAN tatsächlich eine repräsentative Grundlage für die Berechnung der MLU liefern. Auch wenn die Ergebnisse in Kapitel 5 für eine reliable Messung sprechen, könnte in weiteren Forschungsvorhaben parallel die MLU auf Grundlage der Zustimmung von Eltern zu einer größeren Menge vorgegebener Beispielsätze erhoben werden. So könnte die Reliabilität der Messung der MLU geprüft bzw. verbessert werden.

\subsection{Sprechbeginn}

Ist die Verwendung der Variablen Sprechbeginn für die Risikodiagnose bzw. klinische Vorhersage geeignet? Dieses ist eine Frage, die auf Basis vorliegender Ergebnisse nicht beantwortet werden kann, wenn auch die Ergebnisse es als unwahrscheinlich erscheinen lassen, dass Sprechbeginn ein guter Prädiktor für Sprachoutcome im Rahmen des gestörten Spracherwerbs ist. Es könnte dennoch einen kritischen Wert für den Sprechbeginn geben, der für eine Risikodiagnose relevant ist. Solch einen kritischen Wert gelte es an größeren Stichproben mit Extremgruppenbildung zu untersuchen. Für vorliegende Untersuchung hätte sich die Bildung von Extremgruppen aufgrund der kleinen Stichprobe nicht angeboten. So hätte bei einem Cut-off-Wert von 18 Monaten die Extremgruppe aus lediglich vier Kindern bestanden.

Rekursiv für theoretische Spracherwerbsmodelle machen die Ergebnisse zum Sprechbeginn deutlich, wie viel gerade im frühen Alter vor 12 bis 16 Monaten noch unerforscht ist. Was unterscheidet zum Beispiel ein Kind, das über 200 Wörter im rezeptiven Wortschatz verfügt und nicht angefangen hat zu sprechen, von einem Kind welches nur 30 Wörter versteht und schon erste Worte spricht? Welche Wahrnehmungs-, Kognitions- und Gedächtnis- sowie phonologische Fähigkeiten stehen in welchem Zusammenhang, um zur Produktion erster expressiver Sprachäußerung $\mathrm{zu}$ führen? Die Erforschung der Vorläuferfertigkeiten für den expressiven Sprachgebrauch hat sich in den letzten Jahren im Rahmen der Säuglingsforschung deutlich verstärkt (Grimm \& Weinert, 2005) und ermöglichte interessante Einblicke in die Mechanismen des frühen Spracherwerbs. Der 
erzielte Erkenntnisgewinn führte $\mathrm{zu}$ Veränderungen bzw. Erweiterungen bestehender Spracherwerbsmodelle und könnte auch zur Entwicklung von effektiven präventiven Frühinterventionen genutzt werden. Die Vielfalt an ungelösten Fragen und Problemen in Bezug auf den frühen Spracherwerb impliziert die Forderung zur Intensivierung der Sprachforschung für das Säuglingsalter.

\subsection{Qualitative Wortschatzanalyse}

Vor allem für die Ergebnisse in Bezug auf die qualitativen Analysen des frühen Wortschatzes gilt der Wunsch nach Replizierung der Ergebnisse auf Grundlage der critical mass hypothesis, denn die Summe der Funktionswörter steigt z.B. erst ab einem Wortschatzumfang von 200 deutlich an und Verben gelten als besonders guter Prädiktor für die weitere Sprachentwicklung erst ab einer bestimmten Wortschatzgröße. Es sollte eine größere Stichprobe von Kindern nach dem Umfang des expressiven Wortschatzes gruppiert werden, um zu untersuchen, ob qualitative Merkmale für bestimmte Wortschatzgrößen doch zu bedeutsamen Verbesserungen der Vorhersagen späteren Sprachoutcomes aus dem expressiven Wortschatzumfang führen bzw. gar in höherem Zusammenhang zum Sprachoutcome stehen als der frühe Wortschatzumfang.

Auch wäre zu wünschen, die Ergebnisse mit einer größeren Summe z.B. an Verben zu replizieren. Keines der verfügbaren Erhebungsinstrumente für den frühen Wortschatz erhebt jedoch eine ausreichend große Verbenmenge, sodass ergänzende Erhebungsmöglichkeiten entwickelt werden müssten (z.B. eine Verbencheckliste). Die Ergänzung eines bestehenden Verfahrens zur reliableren Erfassung des Verbeninventars in der frühen Kindheit wird zum einen durch den theoretischen Hintergrund fundiert, der die große Bedeutung der Verben für den weiteren Spracherwerb betont. Zum anderen lassen auch die hohen Korrelationen der Summe aller Verben sowie der Summe an Hilfs- und Modalverben mit späterem Sprachoutcome in dieser Arbeit die Bemühungen sinnvoll erscheinen.

Limitierender Faktor für die Wortarten, die zur geschlossenen Klasse der Funktionswörter gehören (wie z.B. die Artikel und Pronomen) ist der Tatbestand, dass in jeder Sprache nur ein begrenztes Inventar an selbigen zur Verfügung steht. Hier können die Prüfmengen in weiteren Untersuchungen nur begrenzt erhöht werden.

Die Variable Verbendefizit könnte Bestand haben mit Hinblick auf einen kritischen Alterswert. An einer größeren Stichprobe mit einem ausreichenden Anteil von klinisch auffälligen Kindern, könnte man untersuchen, ob es ein bestimmtes kritisches Alter gibt, ab dem das Fehlen von Verben $\mathrm{zu}$ reliablen und validen Vorhersagen für die weitere 
Sprachentwicklung auch in Bezug auf die Persistenz einer Sprachentwicklungsverzögerung führt und somit klinische Vorhersagen erlaubt.

Für das Verhältnis von Funktions- zu Inhaltswörtern im frühen Wortschatz (QFI) ergab sich die einzige zusätzliche bedeutsame Varianzaufklärung späteren Sprachoutcomes über den frühen Wortschatz hinaus. Weitere Forschungsbemühungen zu diesem Faktor scheinen lohnenswert. Unbeantwortete Fragen sind unter anderem: Warum gab es nur zu zwei Untertests bedeutsame Korrelationen und warum erlaubte der QFI gerade zum Untertest „Verstehen von Sätzen“ bei den vierjährigen Kindern $\mathrm{zu} \mathrm{t}_{1}$ signifikante zusätzliche Varianzaufklärung? Welche zusätzlichen Informationen trägt der QFI im Vergleich zum expressiven Wortschatzumfang? Oder handelt es sich um ein zufälliges Ergebnis? Neben einer Replizierung der Ergebnisse an einer größeren Stichprobe könnten auch andere Testverfahren zur Erhebung von identischen und weiteren Sprachmaßen zum Einsatz kommen und darüber hinaus könnte die Bedeutung des QFI für andere „rezeptive Leistungen“ der kognitiven Entwicklung geprüft werden. Gibt es bedeutsame Zusammenhänge von QFI und frühen Leistungen bei false belief Aufgaben wäre eine mögliche Forschungsfrage. Die Ergebnisse zum QFI machen deutlich, dass zur Verbesserung der Vorhersagen späteren Sprachoutcomes aus dem frühen Wortschatzumfang sich insbesondere Variablen eignen, die in keinem ausgeprägten Zusammenhang zu selbigem stehen. Es sollte also für weitere Forschungsbemühungen gerade nach den Variablen gesucht werden, die z.B. nicht hoch mit dem Prozentrang im ELAN korrelieren. Dieses könnten auch Variablen aus den sozialanamnestischen Angaben zu Bildungsstand der Eltern, Geschwisterposition oder frühen Auffälligkeiten in der Hörentwicklung der Kinder sein.

Die Anzahl belegter nichtsubstantivischer semantischer Kategorien (Semnisub) verfügte über eine Menge an tendenziell höheren Zusammenhängen zu späterem Sprachoutcome im Vergleich zum expressiven Wortschatzumfang. Insbesondere für diese Variable lohnen weitere Forschungsbemühungen.

Viele inhaltliche Fragen wurden durch die qualitativen Analysen des frühen Wortschatzes aufgeworfen. Interessant wäre, ob es für die Vorhersage späteren Sprachoutcomes tatsächlich egal ist, ob eine semantische Kategorie nur mit einem oder mehreren Items belegt ist. Ab welcher prozentualen Belegung ergeben sich z.B. für die nichtsubstantivischen Kategorien noch bessere Vorhersagen und ist dieses dann doch Ausdruck von „echten Kategorisierungsfähigkeiten“ des Kindes? Und warum erbringen gerade Auxiliar- und Modalverben so eine hohe Anzahl bedeutsamer Korrelationen zu späterem Spachoutcome, wenn man die geringe Menge der Items in Betracht zieht? Gibt es weitere Möglichkeiten, den 
frühen expressiven Wortschatz hinsichtlich qualitativer Aspekte auszuwerten? Gerade die letztgenannten Forschungsfragen betonen die Forderung für weitere Forschungsbemühungen zum Spracherwerb in enger Zusammenarbeit mit anderen Forschungsdisziplinen innerhalb der Psychologie (z.B. Kognitionspsychologie) und mit anderen Fachrichtungen wie z.B. der Linguistik.

Die im Rahmen dieser Arbeit berichteten Ergebnisse gelten nur für die untersuchten sprachlichen Aspekte, die ausnahmslos der linguistischen Kompetenz zuzuordnen sind, und deren Operationalisierungen. Hierbei gibt es unterschiedliche Möglichkeiten sprachliche Aspekte bzw. Komponenten zu unterteilen und zu benennen. Für weitere Forschungsvorhaben könnte als weitere Sprachkomponente der linguistischen Kompetenz die Phonologie in längsschnittliche Betrachtungen einbezogen werden und um Variablen der prosodischen und pragmatischen Kompetenz erweitert werden. Dieses würde Aussagen zum Zusammenwirken der sprachlichen Komponenten und Kompetenzen über ein großes Zeitfenster ermöglichen sowie Information zu Stabilität und prognostischer Validität für selbige ermöglichen. Für die prosodische Kompetenz ist z.B. bekannt, dass sie schon im Säuglingsalter Vorhersagen späterer Sprachleistungen ermöglicht. Von wie guter prognostischer Validität diese Vorhersagen jedoch über eine große Zeitspanne sind, ist noch weitgehend unerforscht.

Entscheidend für den Erkenntnisgewinn $\mathrm{zu}$ den in dieser Arbeit aufgeworfenen Forschungsfragen scheint mir die Operationalisierung der sprachlichen Komponenten zu sein. Diese hängt von den zur Verfügung stehenden Spracherhebungsverfahren ab. Für weitere Studien könnten die sprachlichen Komponenten über vielfältigere Operationalisierungen erfasst werden, um die Ergebnisse auf breiter sprachtheoretischer Basis zu fundieren. Auch wäre es wünschenswert, dass die sprachlichen Leistungen weniger „,arbeitsgedächtnislastig“ erhoben werden. Der SETK 3-5 wurde von vielen Kindern als sehr belastend für ihre Gedächtnisressourcen erlebt, was zum einen die praktische Durchführung schwierig machte, zum anderen aber auch zu einer unerwünschten Konfundierung von Arbeitsgedächtnisleistungen und sprachlichen Leistungen geführt haben kann.

Die Analyse von Entwicklungsstabilitäten und -veränderungen ebenso wie die Vorhersage von Entwicklungsverläufen und die Betrachtung wechselseitiger Beeinflussung verschiedener Entwicklungsbereiche wird erst durch ein längsschnittliches Untersuchungsdesign möglich. Aus diesem Grunde soll der Forderung nach längsschnittlichen Designs in der entwicklungspsychologischen Forschung für zukünftige Forschungsprojekte Nachdruck verliehen werden. Auch wenn in den letzten Jahren an mehreren Standorten in Deutschland 
teilweise sehr umfangreiche Längsschnittstudien initiiert und durchgeführt wurden (u. a. Würzburg, Göttingen und Potsdam), sollte dieser Tatbestand erst als Anfang einer erfreulichen Entwicklung verstanden werden und zu verstärkten Bemühungen gerade mit Hinblick auf die Erforschung des Spracherwerbs motivieren. 


\section{Zusammenfassung}

Im Rahmen dieser Längsschnittuntersuchung zum Spracherwerb wurden im frühen Kindesalter von 16 bis 26 Monaten an 57 deutschsprachigen Kindern der frühe expressive Wortschatz sowie grammatische Leistungen erhoben und mit den späteren Wortschatz-, Grammatik- und Satzgedächtnisleistungen im mittleren Alter von 4;0 Jahren, 5;0 Jahren und 7;10 Jahren $(n=53)$ in Beziehung gesetzt.

Neben den Entwicklungsstabilitäten von Sprache interessierte vor allem die prädiktive Validität des frühen Wortschatzes für späteres Sprachoutcome bis ins Grundschulalter und es wurde geprüft, ob frühe grammatische Fähigkeiten sowie qualitative Analysen des frühen Lexikons zu substanziellen Verbesserungen dieser Vorhersagen aus dem expressiven Wortschatzumfang beitragen können. Zudem wurde der Sprechbeginn auf das Bestehen bedeutsamer Zusammenhänge zu späteren Sprachleistungen betrachtet.

Sprache zeigte sich in Übereinstimmung mit der aktuellen Forschungsliteratur als Merkmal von mehrheitlich mittlerer bis hoher differentieller Stabilität für die Altersspanne vom 2. bis 8. Lebensjahr, wobei die Zeitspanne zwischen den Erhebungszeitpunkten und der Wechsel von Erhebungsverfahren die Ausprägung der Stabilität beeinflusste.

Der frühe expressive Wortschatz erlaubte gute Vorhersagen für Wortschatz-, Grammatikund Satzgedächtnisleistungen im Vorschulalter. Für das Grundschulalter waren die Varianzaufklärungsbeiträge niedrig und nicht für alle Sprachmaße ließen sich substanzielle Vorhersagen aus den frühen Wortschatzdaten ableiten.

Bedeutsame Verbesserungen der Vorhersagen späteren Sprachoutcomes aus dem frühen expressiven Wortschatzumfang ergaben sich für die frühen grammatischen Fähigkeiten nicht.

Das Alter, in dem erste Worte gesprochen werden, stand in keinem bedeutsamen Zusammenhang zu späteren Sprachleistungen.

Die qualitativen Analysen des frühen Wortschatzes erbrachten keine bedeutsamen Verbesserungen der Vorhersage späteren Sprachoutcomes aus dem expressiven Wortschatzumfang. Trotz des mehrfachen Vorliegens tendenziell höherer Zusammenhänge von qualitativen Wortschatzaspekten und Sprachoutcome, ließen sich keine substanziell höheren Zusammenhänge zu späterem Sprachoutcome im Vergleich zur bewährten quantitativen Analyse des Wortschatzes feststellen. 


\section{Literaturverzeichnis}

Au, T. K., Dapretto, M. \& Song, Y. K. (1994). Input vs. Constraints: Early word acquisition in Korean and English. Journal of Memory and Language, 33, 567-582.

Bassano, D., Eme, P. \& Champaud, C. (2005). A naturalistic study of early lexical development: General processes and interindividual variations in French children. First Language, 25, 67-101.

Bates, E. (1993). Comprehension and production in early language development. Monographs of the Society for research in Child Development, 58, 222-242; discussion 243-252.

Bates, E. (1994). Parents as scientific observers of their children`s development. In S.L. Friedman \& H.C. Haywood (Eds). Developmental follow-up. Concepts, domains, and methods. San Diego, California: Academic Press, Inc.

Bates, E., Beeghly, M., Bretherton, I., McNew, S., O`Connell, B., Reznick, S. et al. (1984). Early Language Inventory. Unpublished manuscript, Center for Research on Language, University of California, SD.

Bates, E. Bretherton, I. \& Snyder, L. (1988). From first words to grammar. Individual differences and dissociable mechanisms. Cambridge: Cambridge University Press.

Bates, E. \& Carnevale, G. F. (1993). New directions in research on language development. Developmental Review, 13, 436-470.

Bates, E., Marchman, V., Thal, D., Fenson, L., Dale, P., Reznick et al. (1994). Developmental and stylistic variations in the composition of early vocabulary. Journal of Child Language, 21, 85-124.

Behrens, H. (1999). Was macht Verben zu einer besonderen Kategorie im Spracherwerb? In: J. Meibauer \& M. Rothweiler (Hrsg.). Das Lexikon im Spracherwerb (S. 32-50). Tübingen: Francke. 
Behrens, H. (2002). Das Verb im Spracherwerb. Forum Logopädie, 3, 16-21.

Benedict, H. (1979). Early lexical development: comprehension and production. Journal of Child Language, 6, 183-200.

Berglund, E. \& Eriksson, M. (2000). Communicative development in Swedish children 16-28 months old: The Swedish early communicative development inventory - word and sentences. Scandinavian Journal of Psychology, 41, 133-144.

Blake, J., Quartaro, G. \& Onorati, S. (1993). Evaluating quantitative measures of grammatical complexity in spontaneous speech samples. Journal of Child Language, 20, 139-152.

Bloom, L., Tinker, E. \& Margulis, C. (1993). The words children learn: Evidence against a noun bias in early vocabularies. Cognitive development, 8, 431-450.

Bockmann, A. (2001). Konstruktion und Entwicklung eines Verfahrens zur Erfassung des expressiven Wortschatzes in der frühen Kindheit. Unveröffentlichte Diplomarbeit. Georg-August-Universität Göttingen.

Bockmann, A. \& Kiese-Himmel, C. (2006). Elan - Eltern Antworten. Elternfragebogen zur Wortschatzentwicklung im frühen Kindesalter. 1. Aufl. Göttingen: Beltz.

Bornstein, M. H., Hahn, C. \& Haynes O. M. (2004). Specific and general language performance across early childhood: Stability and gender considerations. First language, 24, 267-304.

Bornstein, M. H. \& Haynes, O. M. (1998). Vocabulary competence in early childhood: Measurement, latent construct, and predictive validity. Child Development, 69, 654671.

Bornstein, M. H., Haynes, O. M., Painter, K. M. \& Genevro, J. L. (2000). Child language with mother and with stranger at home and in the laboratory: a methodological study. Journal of Child Language, 27, 407-420. 
Bornstein, M. H., Putnick, D. L. \& De Houwer, A. (2006). Child vocabulary across the second year: Stability and continuity for reporter comparisons and a cumulative score. First Language, 26, 299-316.

Braine, M. D. S. (1963). On learning the grammatical order of words. Psychological Review, 70, 323-348.

Brown, R. (1973). A first language: The early stages. Cambridge, MA: Harvard University Press.

Bühler, C. (1928). Kindheit und Jugend. Leipzig: Hirzel.

Buschmann, A., Jooss, B., Blaschtikowitz, H., Koch-Graus, A., Schumacher, D., Heggen, I. et al. (2007). Frühe Sprachförderung. In D. Karch \& J. Pietz (Hrsg.), Aktuelle Neuropädiatrie 2006 (S. 79-85). Nürnberg: Novartis Pharma Verlag.

Bußmann, H. (2002). Lexikon der Sprachwissenschaft. 3. erweiterte und überarb. Aufl. Stuttgart: Alfred Kröner Verlag.

Caselli, M. C., Bates, E., Casadio, P., Fenson, J., Fenson, L., Sanderl, L. \& Weir, J. (1995). A cross-linguistic study of early lexical development. Cognitive Development, 10, 159199.

Cheng, L. (2000). Children of yesterday, today and tomorrow: Global implications for child language. Folia Phoniatrica et Logopaedica, 52, 39-47.

Choi, S. \& Gopnik, A. (1995). Early acquisition of verbs in Korean: A cross-linguistic study. Journal of Child Language, 22, 497-529.

Cohen, J. (1988). Statistical power analysis for the behavioural sciences. Hillsdale, NJ: Erlbaum. 
Conboy, B. T. \& Thal, D. J. (2006). Ties Between the Lexicon and Grammar: Cross-Sectional and Longitudinal Studies of Bilingual Toddlers. Child Development, 77, 712-735.

Conti-Ramsden, G. (2003). Factors influencing word learning in young children with SLI. Vortrag auf dem 5. Europäischen Kongress des "Comité Permanent de liaison des Orthophonistes / Logopèdes de l'union Européenne" (CPLOL) vom 5. bis 7. September 2003 in Edinburgh.

Conti-Ramsden, G. \& Jones, M. (1997). Verb use in specific language impairment. Journal of Speech, Language, and Hearing Research, 40, 1298-1313.

Dale, P. S. (1990). Parent report and the growth of $M L U$. Unpublished manuscript.

Dale, P. S. (1991). The validity of a parent report measure of vocabulary and syntax at 24 months. Journal of Speech and Hearing Research, 34, 565-571.

Dale, P. S., Bates, E., Reznick, J. S. \& Morisset, C. (1989). The validity of a parent report instrument of child language at twenty months. Journal of Child Language, 16, 239249.

Dale, P. S., Price, T. S., Bishop, D. V. M. \& Plomin, R. (2003). Outcomes of early language delay: Predicting persistent and transient language difficulties at 3 and 4 years. Journal of Speech, Language and Hearing research, 46, 544-560.

Deutsches Jugendinstitut. (2004). Expertise zu Sprachstandserhebungen für Kindergartenkinder und Schulanfänger. Eine kritische Betrachtung von Lilian Fried. Zugriff am 13.1.2007 http://www.dji.de/bibs/271_2232_ExpertiseFried.pdf

Dijkstra, T. \& Kempen, G. (1993). Einführung in die Psycholinguistik. Übersetzt aus dem Niederländischen von Y. H. W. Fuchs. Bern: Huber.

Dionne, G., Dale, P. S., Boivin, M. \& Plomin, R. (2003). Genetic Evidence for Bidirectional Effects of Early Lexical and Grammatical Development. Child Development, 74, 394412. 
Dudenredaktion (Hrsg.) (2001). Duden: Deutsches Universalwörterbuch. 4. Aufl. Mannheim: Dudenverlag.

Dudenredaktion (Hrsg.) (2006). Deutsche Grammatik - kurz gefasst. 3. überarbeitete Aufl. Mannheim: Dudenverlag.

Dunn, L. W. \& Dunn, L. M. (1981). Peabody Picture Vocabulary Test-Revised (Forms L and M). Circle Pines, MN: American Guidance Service Inc.

Dunn, L. W. \& Dunn, L. M. (1997). Peabody Picture Vocabulary Test, $3^{\text {rd }}$ ed. Circle Pines, MN: American Guidance Service Inc.

Eggert, D. \& Schuck, K. D. (1975). Hannover-Wechsler-Intelligenztest für das Vorschulalter. Bern: Verlag Hans Huber.

Feldman, H. M., Campbell, T. F., Colborn, D. K., Dale, P. S., Kurs-Lasky, M., Paradise, J. L. et al. (2005). Concurrent and Predictive Validity of Parent Reports of Child Language at Ages 2 and 3 Years. Child Development, 76, 856-868.

Feldman, H. M., Dollaghan, C. A., Cambell, T. F., Kurs-Lasky, M., Janosky, J. E. \& Paradise, J. L. (2000). Measurement properties of the Mac Arthur Communicative development inventories at ages one and two years. Child Development, 71, 310-322.

Fenson, L., Dale, P. S., Reznick, J. S., Bates, E., Thal, D. \& Pethick, J. S. (1994). Variability in early communicative development. Monographs of the Society for research in Child Development, Serial No. 242.

Fenson, L., Dale, P. S., Reznick, J. S., Thal, D., Bates, E., Hartung, J. P. et al. (1993). Mac Arthur Communicative Development Innventories. User's Guide and Technical Manual. (2 ${ }^{\text {nd }}$ Print, 1996) San Diego, CA: Singular Publishing Group Inc.

Fischel, J. E., Whitehurst, G. J., Caulfield, M. B. \& DeBaryshe, B. (1989). Language growth in children with expressive language delay. Pediatrics, 82, 218-227. 
Francescato, G. (1973). Spracherwerb und Sprachstruktur beim Kinde. Stuttgart: Klett.

Fromm, W. \& Schöler, H. (1997). Arbeitsgedächtnis und Sprachentwicklung. Untersuchung an sprachentwicklungsauffälligen und sprachunauffälligen Schulkindern (Arbeitsbericht aus dem Forschungsprojekt "Differentialdiagnostik", Nr. 3). Heidelberg: Pädagogische Hochschule, Erziehungs- und sozialwissenschaftliche Fakultät.

Gavin, W. J. \& Giles, L. (1996). Sample size effects on temporal stability of language sample measures of preschool children. Journal of Speech and Hearing Research, 39, 12581262.

Goldfield, B. A. \& Reznick, J. S. (1990). Early lexical acquisition: Rate, content, and the vocabulary spurt. Journal of Child Language, 17, 171-183.

Goorhuis-Brouwer, S. M. (1990). Frühzeitige Erkennung von Sprachentwicklungsstörungen. Die Eltern als engagierte Beobachter von Sprachentwicklungsstörungen. Folia Phoniatrica, 42, 260-264.

Grimm, H. (1995a). Spezifische Störungen der Sprachentwicklung. In R. Oerter \& L. Montada (Hrsg.), Entwicklungsspychologie (S. 943-953). Weinheim: Psychologie Verlags Union.

Grimm, H. (1995b). Sprachentwicklung-allgemeintheoretisch und differentiell betrachtet. In R. Oerter \& L. Monatada (Hrsg.), Entwicklungspsychologie (S. 705-757). Weinheim: Psychologie Verlags Union.

Grimm, H. (1999). Störungen der Sprachentwicklung. Göttingen: Hogrefe.

Grimm, H. (unter Mitarbeit von M. Aktas und S. Frevert) (2000). Sprachentwicklungstest für zweijährige Kinder (SETK-2). Diagnose rezeptiver und produktiver Sprachverarbeitungsfähigkeiten. Manual. Göttingen: Hogrefe. 
Grimm, H. (2001). Sprachentwicklungstest für drei- bis fünfjährige Kinder. Göttingen: Hogrefe.

Grimm, H. \& Doil, H. (2000). Elternfragebögen für die Erfassung von Risikokindern (ELFRA). Göttingen: Hogrefe.

Grimm, H., Doil, H., Müller, C. \& Wilde, S. (1996). Elternfragebogen für die differentielle Erfassung früher sprachlicher Fähigkeiten. Sprache \& Kognition, 15, 32-45

Grimm, H. \& Schöler, H. (1991). Heidelberger Sprachentwicklungstest. 2. verbesserte Auflage. Göttingen: Hogrefe.

Grimm, H. \& Weinert, S. (2005) Sprachentwicklung. In R. Oerter \& L. Monatada (Hrsg.), Entwicklungspsychologie (S. 517-550). Weinheim: Psychologie Verlags Union.

Harris, M., Yeeles, C., Chasin, J. \& Oakley, Y. (1995). Symmetries and asymmetries in early lexical comprehension and production. Journal of Child Language, 22, 1-18.

Hart, B. \& Risley, T. R. (1989). The longitudinal study of interactive systems. Education and Treatment of Children, 12, 347-358.

Hellbrügge, T. (1994). Münchener Funktionelle Entwicklungsdiagnostik: 2. und 3. Lebensjahr (4. korr. und erw. Aufl.). München: Dienstleistungsgesellschaft für gemeinnützige Institutionen.

Höhle, B. (2004). Sprachwahrnehmung und Spracherwerb im ersten Lebensjahr. Sprache Stimme Gehör, 28, 2-7.

Jansen, H., Mannhaupt, G., Marx, H. \& Skowronek, H. (2002). Bielefelder Screening zur Früherkennung von Lese-Rechtschreibschwierigkeiten. 2. überarbeitete Auflage. Göttingen: Hogrefe. 
Johnston, J., Miller, J. F., Curtiss, S. \& Tallal, P. (1993). Conversations with children who are language impaired: Asking questions. Journal of Speech and Hearing Research, 36, 973-978.

Kamhi, A. G. (1986). The elusive first word: The importance of the naming insight for the development of referential speech. Journal of Child Language, 13, 155-161.

Kauschke, C. (2003). Entwicklung, Störungen und Diagnostik lexikalischer Prozesse Wortverständnis und Wortproduktion. Sprache Stimme Gehör, 27, 110-118.

Kelly, D.J. (1997). Patterns in verb use by preschoolers with normal language and specific language impairment. Applied Psycholinguistics, 18, 199-218.

Kiese, C. \& Kozielski P. M. (1996). Aktiver Wortschatztest für 3-6-jährige Kinder. (2. überarbeitete Auflage. Göttingen: Beltz Verlag.

Kiese-Himmel, C. (1999). Hörgestörte Kinder und ihr Spracherwerb. Eine empirische Analyse. Heidelberg: Median Verlag von Killisch-Horn GmbH.

Kiese-Himmel, C. (2005). Aktiver Wortschatztest für 3- bis 5-jährige Kinder, Revision, 1. Auflage. Göttingen: Beltz Verlag.

Kim, M., McGregor, K. K. \& Thompson, C. K. (2000). Early lexical development in Englishand Korean-speaking children: Language-general and language-specific patterns. Journal of Child Language, 27, 225-254.

King, G. \& Fletcher, P. (1993). Grammatical problems in school-age children with language impairment. Clinical Linguistics and Phonetics, 7, 339-352.

Knapp, K., Antas, G., Becker-Mrotzek, M., Deppermann, A., Göpferich, S., Grabowski, J. et al. (2004). Angewandte Linguistik: Ein Lehrbuch. S. 507-525. Tübingen: Frencke. 
Krajewski, K., Kron, V. \& Schneider, W. (2004). Entwicklungsveränderungen des strategischen Gedächtnisses beim Übergang vom Kindergarten in die Grundschule. Zeitschrift für Entwicklungspsychologie und Pädagogische Psychologie, 36, 47-58.

Kupfer, H. (1959). Vom Aufbau des kindlichen Wortschatzes. Wirkendes Wort, 9, 291-300.

Law, J., Boyle, J., Harris, F. Harkness, A. \& Nye, C. (1998). Screening for speech and language delay: A systematic review of the literature. Health Technology Assessment, 2.

Law, J., Boyle, J., Harris, F. Harkness, A. \& Nye, C. (2000). The feasibility of universal screening for primary speech and language delay: Findings from a systematic review of the literature. Developmental Medicine \& Child Neurology, 42, 190-200.

Lockl, K., Schwarz, S. \& Schneider, W. (2004). Sprache und Theory of mind: Eine Längsschnittstudie bei Drei- bis Vierjährigen. Zeitschrift für Entwicklungspsychologie, $36,207-220$.

Lockl, K. \& Schneider, W. (2007). Knowledge about the mind: Links between the theory of mind and later metamemory. Child development, 78, 148-167.

Maital, S., Painter, K., Park, S., Pascual, L., Pêcheux, M., Ruel, J. et al. (2004). CrossLinguistic Analysis of Vocabulary in Young Children: Spanish, Dutch, French, Hebrew, Italian, Korean, and American English. Child Development, 75, 1115-1139.

Marchman, M. A. \& Bates, E. (1994). Continuity in lexical and morphological development: a test of the critical mass hypothesis. Journal of Child Language, 21, 339-366.

Marx, P. (2006, Januar). Daten aus der Studie „Sprachentwicklung und Schriftspracherwerb“. Referat für den Workshop „Bildungspanel“ in Bamberg.

McCarthy, D. (1966). Language development in children. In L. Carmichael (Ed.), The acquisition of language. New York: Harper. 
McGregor, K. K., Sheng, L. \& Smith, B. (2005). The precocious two-year-old: status of the lexicon and links to grammar. Journal of Child Language, 32, 563-585.

Melchers, P. \& Preuß, U. (1991). Kaufmann-Assessment Battery for Children (K-ABC). Deutschsprachige Fassung. Amsterdam: Swets \& Zeitinger.

Miller, G. A. (1991). The Science of Words. New York: The Scientific American Library.

Nelson, K. (1973). Structure and strategy in learning to talk. Monographs of the Society for Research in Child development, 38 (1-2, Serial No. 149).

Nelson, K. (1988). Constraints on word Learning? Cognitive Development, 3, 221-246

Newcomer, P. L. \& Hammill, D. D. (1988). Test of Language Development - 2: Primary. Austin, TX: Pro-Ed.

Nice, M. M. (1925). Length of sentences as a criterion of a child`s progress in speech. Journal of Educational Psychology, 16, 370-379.

Pan, B. A., Rowe, M. L., Spier, E. \& Tamis-Lemonda, C. (2004). Measuring productive vocabulary of toddlers in low-income families: concurrent and predictive validity of three sources of data. Journal of Child Language, 31, 587-608

Parker, M. D. (2005). A comparative study between mean length of utterance in morphemes (MLUm) and mean length of utterance in words (MLUw). First Language, 25, 365376.

Paul, R. (1993). Patterns of development in late talkers: Preschool years. Journal of Childhood Communication Disorders, 15, 7-14.

Paul, R. (2000). Predicting outcomes of early expressive language delay: ethical implications. In: D. Bishop \& L. B. Leonard (Eds.). Speech and language impairments in children. (S. 195-210). Hove: Psychology Press. 
Paul, R. \& Kellogg, L. (1997). Temperament in late talkers. Journal of Child Psychology and Psychiatry, 38, 803-811.

Pine, J. M., Lieven, E. V. \& Rowland, C. (1996). Observational and checklist measures of vocabulary composition: What do they mean? Journal of child language, 23, 573-590.

Prathanee, B., Thinkhamrop, B. \& Dechongkit, S. (2006). Journal of The Medical Association of Thailand, 89, 1775-1787.

Pruden, S. M., Hirsh-Pasek, K., Michnik Golingkoff, R. \& Hennon, E. A. (2006). The Birth of Words: Ten-Month-Olds Learn Words Through Perceptual Salience. Child Development, 77, 266-280.

Reese, E. \& Read, S. (2000). Predictive Validity of the New Zealand Mac Arthur Communicative Development Inventories: Words and Sentences. Journal of Child Language, 27, 255-266.

Rescorla, L. (1989). The language development survey: A screening tool for delayed language in toddlers. Journal of Speech and Hearing Disorders, 54, 587-599.

Rescorla, L. (1997). Late talkers at 2: Outcome at age 3. Journal of Speech, Language, and Hearing Research, 40, 556-566.

Rescorla, L. (2002). Language and reading outcomes to age 9 in late-talking toddlers. Journal of Speech, Language, and Hearing Research, 45, 360-371.

Rescorla, L. (2005). Age 13 language and reading outcomes in late-talking toddlers. Journal of Speech, Language, and Hearing Research, 48, 459-472.

Rescorla, L., Hadicke-Wiley, M. \& Escarce, E. (1993). Epidemiological investigation of expressive language delay at age two. First Language, 13, 5-22.

Rescorla, L., Mirak, J. \& Singh, L. (2000). Vocabulary growth in late talkers: Lexical development from 2;0 to 3;0. Journal of Child Language, 27, 293-311. 
Reynell, J. S. \& Huntley, M. (1985). Reynell Developmental Language Scales (2 ${ }^{\text {nd }}$ revision). Windsor, England: NFER-Nelson.

Rice, M. L., Redmond, S. M. \& Hoffman, L. (2006). Mean Length of Utterance in Children with Specific Language Impairment and in Younger Control Children Shows Concurrent Validity and Stable and Parallel Growth Trajectories. Journal of Speech, Language, and Hearing Research, 49, 793-808.

Roberts, B. W. \& DelVecchio, W. F. (2000). The rank-order consistency of personality traits from childhood to old age: A quantitative review of longitudinal studies. Psychological Bulletin, 126, 3-25.

Ruff, H. A., Lawson, K. R., Parrinello, R. \& Weissberg, R. (1990). Long-term stability of individual differences in sustained attention in the early years. Child Development, 61, $60-75$.

Sachse, S. (2005). Früherkennung von Sprachentwicklungsstörungen. In W. v. Suchodoletz (Hrsg.), Früherkennung von Entwicklungsstörungen (S. 155-189). Göttingen: Hogrefe.

Schneider, W., Lockl, K. \& Fernandez, O. (2005). Interrelationships Among Theory of Mind, Executive Control, Language Development, and Working Memory in Young Children: A Longitudinal Analysis. In W. Schneider, R. Schumann-Hengsteler \& B. Sodian (Eds.), Young children's cognitive development: Interrelations among executive functioning, working memory, verbal ability, and theory of mind (S. 259284). Hillsdale, NJ: Lawrence Erlbaum Associates.

Silva, P. A. (1980). The prevalence, stability and significance of developmental language delay in preschool children. Developmental Medicine and Child Neurology, 2, 768777.

Snyder, L. S., Bates, E. \& Bretherton, I. (1981). Content and context in early lexical development. Journal of Child Language, 8, 565-582. 
Sparrow, S. S., Balla, D. A. \& Cicchetti, D. V (1984). Vineland Adaptive Behaviour Scales: Interview Edition, Survey Form. Circle Pine, MN: American Guidance Service.

Spiel, C., Schabmann, A. \& Glück, J. (in Druck). Entwicklungsstabilitäten. In M. Hasselhorn und W. Schneider (Hrsg.): Handbuch der Entwicklungspsychologie. Göttingen: Hogrefe.

Stern, C. \& Stern, W. (1907, 1922). Die Kindersprache: Eine psychologische und sprachtheoretische Untersuchung (1., 3. Aufl.). Leipzig: Barth.

Szagun, G. (1996). Sprachentwicklung beim Kind (6. Aufl.). Weinheim: Beltz Verlag.

Szagun, G. (2001). Wie Sprache entsteht. Spracherwerb bei Kindern mit beeinträchtigtem und normalem Hören. Weinheim: Beltz Verlag.

Szagun, G. \& Steinbrink. C. (2004). Typikabilität und Variabilität in der frühkindlichen Sprachentwicklung: eine Studie mit einem Elternfragebogen. Sprache Stimme Gehör, $28,137-145$.

Tamis-LeMonda, C. S. \& Bornstein, M. H. (1994). Specificity in mother-toddler languageplay relations across the second year. Developmental Psychology, 30, 283-292.

Tamis-LeMonda, C. S., Bornstein, M. H., Kahana-Kalman, R., Baumwell, L. \& Cyphers, L. (1998). Predicting variation in the timing of language milestones in the second year: An events history approach. Journal of Child Language, 2, 675-700.

Tewes, U., Rossman, P. \& Schallberger, U. (1999). Hamburg-Wechsler-Intelligenztest für Kinder III. 1. Aufl. Bern: Verlag Hans Huber

Thal, D. J., Bates, E., Zappia, M. J. \& Oroz, M. (1996). Ties between lexical and grammatical development: Evidence from early talkers. Journal of Child Language, 23, 349-368.

Tomasello, M. (1992). First verbs: A study of early grammatical development. Cambridge, UK: Cambridge University Press. 
Tomasello, M. \& Mervis, C. B. (1994). Commentary: the instrument is great, but measuring comprehension is still a problem. In: L. Fenson, P. S. Dale, J. S. Reznick, E. Bates, D. J. Thal \& S. J. Pethick, Monographs of the Society for Research in Child development, Serial No. 242, Vol. 59, 174-179.

Tomblin, J. B., Records, N. L., Buckwalter, P., Zhang, X., Smith, E. \& O`Brien, M. (1997). Prevalence of specific language impairment in kindergarten children. Journal of Speech, Language, and Hearing Research, 40, 1245-1260.

Tomblin, J. B., Zhang, X., Buckwalter, P. \& O`Brien, M. (2003). The stability of primary language disorder: four years after kindergarten diagnosis. Journal of Speech, Language, and Hearing Research, 46, 1283-96.

Toppelberg, C. O. \& Shapiro, T. (2000). Language disorders: A 10-year research update review. Journal of the American Academy of Child and Adolescent Psychiatry, 39, 143-152.

Trier, J. (1973). Der deutsche Wortschatz im Sinnbezirk des Verstandes: von den Anfängen bis zum Beginn des 13. Jahrhunderts. Heidelberg: Winter (Originalarbeit erschienen 1931).

Vogt, S. (2003). Vergleich standardisierter Testverfahren zur semantisch-lexikalischen Entwicklung: Was wird eigentlich gemessen? Sprache Stimme Gehör, 27, 119-124.

Walker, D., Greenwood, B. H. \& Carta, J. (1994). Prediction of School Outcomes based on Early Language Production and Socioeconomic Factors. Child Development, 65, 606621.

Ward, S. (1999). An Investigation into the effectiveness of an early intervention method for delayed language development in young children. International Journal of Language and Communication Disorders, 34, 243-264. 
Weber, J., Marx, P. \& Schneider, W. (2005, September). Zur Stabilität von Wortschatz, Grammatik und phonologischer Informationsverarbeitung im Alter von 4 bis 8 Jahren. Poster präsentiert auf der 17. Tagung Entwicklungspsychologie in Bochum.

Wechsler, D. (1967). Preschool and Primary Scale of Intelligence - Revised. New York: Psychological Corp.

Wechsler, D. (1989). Wechsler Preschool and Primary Scale of Intelligence - Revised Manual. San Antonio, TX: The Psychological Corporation.

Weindrich, D., Jennen-Steinmetz, C., Rellum, T., Laucht, M. \& Schmidt, M. H. (2005). Sprachentwicklungsstand mit 10 Monaten. Prognostische Validität für spätere Sprachentwicklungsdefizite. Monatsschrift Kinderheilkunde, 153, 150-156.

Weinert, F. E. \& Helmke, A. (1993). Wie bereichsspezifisch verläuft die kognitive Entwicklung? In R. Duit \& W. Gräber (Hrsg.), Kognitive Entwicklung und Lernen der Naturwissenschaften (S. 27-45). Kiel: Institut für die Pädagogik der Naturwissenschaften (IPN).

Weinert, S. (2004). Wortschatzerwerb und kognitive Entwicklung. Sprache Stimme Gehör, 28, 20-28.

Wengenrath, A. (2006). Die kortikale Verarbeitung semantischer Domänen: eine ERP-Studie. Zugriff am 13.5.2007 http://bieson.ub.uni-bielefeld.de/volltexte/2006/841

Westerlund, M., Berglund, E. \& Eriksson, M. (2006). Can Severely Language Delayed 3Year-Olds Be Identified at 18 months? Evaluation of a Screening Version of the Mac Arthur-Bates Communicative Development Inventories. Journal of Speech, Language, and Hearing Research, 49, 237-247.

Williams, K. T. (1997). Expressive Vocabulary Test. Circle Pines, MN: American Guidance Service.

Zimmer, D. E. (1988). So kommt der Mensch zur Sprache. Zürich: Haffmans Verlag. 


\section{Tabellenverzeichnis}



2. Altersverteilung der Untersuchungsstichprobe zum Zeitpunkt $t_{1} \ldots \ldots \ldots \ldots \ldots \ldots$

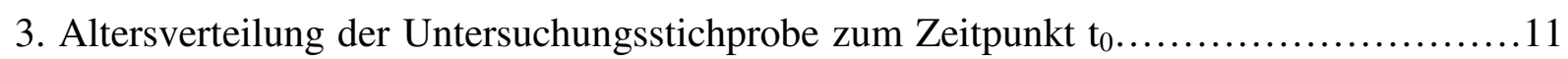

4. Altersverteilung der Untersuchungsstichprobe zum Zeitpunkt $t_{2} \ldots \ldots \ldots \ldots \ldots \ldots \ldots \ldots \ldots \ldots \ldots \ldots \ldots$

5. Altersverteilung der Untersuchungsstichprobe zum Zeitpunkt $t_{3} \ldots \ldots \ldots \ldots \ldots \ldots \ldots \ldots \ldots$

6. Beschreibung des Untersuchungsplans........................................ 14

7. Überblick über die verwendeten Untersuchungsverfahren von $t_{0}$ bis $t_{3} \ldots \ldots \ldots \ldots \ldots \ldots$

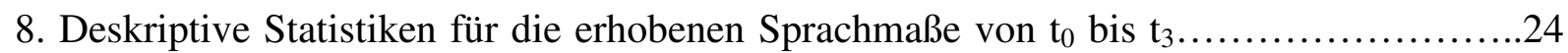

9. Testergebnisse für die Prüfung der Mittelwertsdifferenzen über alle Sprachmaße von $t_{1} z u$

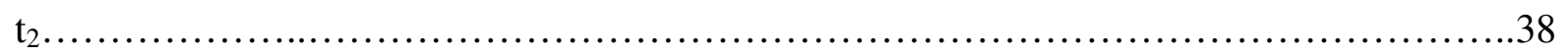

10. Vorhersagen späterer Sprachleistungen von $t_{1}$ bis $t_{3}$ aus dem frühen Wortschatz........62

11. Zusammenhänge früher grammatischer Fähigkeiten und früher Wortschatzleistunge $\left(\mathrm{zu} \mathrm{t}_{0}\right)$



12. Zusammenhänge zwischen Sprechbeginn, frühem Wortschatz und den Sprachmaßen von

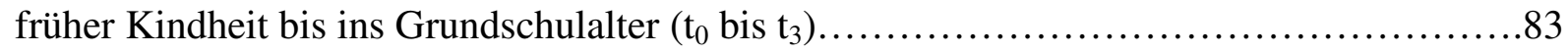

13. Zusammenhänge von Wortartenvariablen (Verben) und Gesamtwortschatzumfang mit

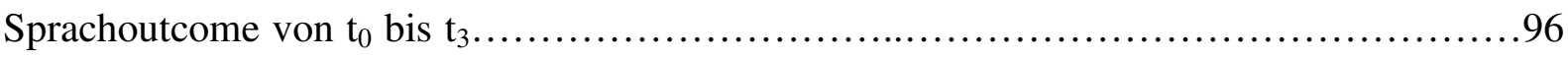

14. Zusammenhänge weiterer Wortartenvariablen (außer Verben) und des

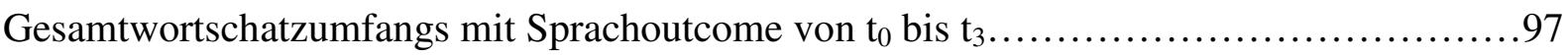

15. Zusammenhänge von Funktionswörtern, dem Verhältnis von Funktions- und Inhaltswörtern sowie dem Gesamtwortschatzumfang mit Sprachoutcome von $t_{0}$ bis $t_{3} \ldots . .105$ 16. Zusammenhänge von Anzahl der semantischen Kategorien und Gesamtwortschatzumfang mit Sprachoutcome von $t_{0}$ bis $t_{3}$.... 


\section{Abbildungsverzeichnis}

1. Zusammenhänge von Wortschatzleistungen (WS) und expressiven grammatischen Fähigkeiten (MLU und Pluralsumme) $\mathrm{zu} \mathrm{t}_{0}$

2. Zusammenhänge von Wortschatzleistungen, expressiven grammatischen Fähigkeiten (MR) und rezeptiven grammatischen Fähigkeiten (VS) für die dreijährigen Kinder $\mathrm{zu} \mathrm{t}_{1} \ldots \ldots \ldots . . .35$ 3. Zusammenhänge von Wortschatzleistungen, expressiven grammatischen Fähigkeiten (MR), rezeptiven grammatischen Fähigkeiten (VS) und Satzgedächtnisfertigkeit (SG) für die



4. Zusammenhänge von Wortschatzleistungen (WS), expressiven grammatischen Fähigkeiten (MR), rezeptiven grammatischen Fähigkeiten (VS) und Satzgedächtnisleistungen (SG) zu

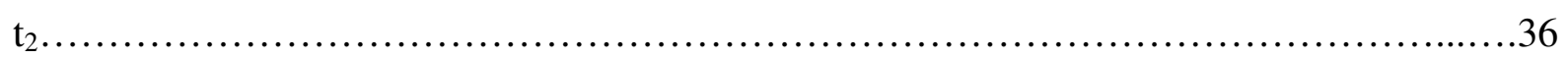

5. Zusammenhänge von Wortschatzleistungen (WS), expressiven grammatischen Fähigkeiten (MR), rezeptiven grammatischen Fähigkeiten (VS) und Satzgedächtnisleistungen (SG) zu

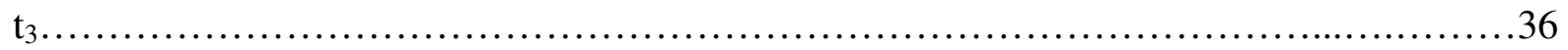

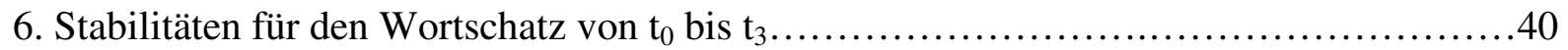

7. Stabilitäten für die rezeptiven grammatischen Leistungen von $t_{1}$ bis $t_{3} \ldots \ldots \ldots \ldots \ldots \ldots . \ldots 1$

8. Stabilitäten für die expressiven grammatischen Leistungen von $t_{0}$ bis $t_{3} \ldots \ldots \ldots \ldots \ldots . \ldots 2$






\section{Anhangsverzeichnis}
A. Elternfragebogen zur Wortschatzentwicklung (vorläufige Endform) 143
B. Anamnesebogen $t_{1}$ 149
C. Erweiterung des Anamnesebogens zu t $t_{2}$. 151
D. Elternfragebogen $t_{3}$. 155 


\section{Anhang}

Anhang A

Elternfragebogen zur Wortschatzentwicklung des Kindes (vorläufige Endform)

\section{Elternfragebogen zur Wortschatzentwicklung des Kindes}

\section{Wir bitten Sie, bei den folgenden Fragen entweder das zutreffende Kästchen anzukreuzen oder die Antwort auf die gepunktete Linie zu schreiben.}

Heutiges Datum:

\section{Fragen zum Kind}

Junge $\square \quad$ Mädchen $\square$

Geburtsdatum:

Wie viele Geschwister, die in der Familie leben, hat Ihr Kind?


Zwilling: $\quad$ ja $\square$ nein $\square$

War die Geburt auffällig? nein $\square$ ja $\square$ Wenn ja, was war auffällig:

Hat Ihr Kind in den letzten 12 Monaten 3 oder mehr Mittelohrentzündungen gehabt? ja $\square$ nein $\square$

Ist Ihr Kind in einer Krabbelgruppe, Spielkreis, PEKiP-Gruppe, Musikgarten o.ä.?

ja $\square$ nein $\square$

Ca. wie viele Stunden in der Woche ist Ihr Kind dort insgesamt?

bis 4 Stunden

5 bis 10 Stunden

$\square$

11 bis 20 Stunden

a

mehr als 20 Stunden

Seit wann ist Ihr Kind dort?

Datum:

Wann sprach Ihr Kind erste Worte (keine Kindersprache wie "wauwau")?

mit ..... Monaten

Haben Ihre anderen Kinder Schwierigkeiten mit der Sprachentwicklung gehabt?

entfällt $\square$ nein $\square$ ja Wenn ja, welche Geschwister mit welchen Problemen:

\section{Fragen zu den Vorsorgeuntersuchungen}

Bitte geben Sie für die bereits durchgeführten Vorsorgeuntersuchungen (U1 - U7) an, ob es dort Auffälligkeiten gab:

U1 (direkt nach der Geburt) auffälliga unauffälliga

wenn auffällig, was:

U2 (3. - 10. Lebenstag)

auffällig

unauffällig 
wenn auffällig, was:

U3 (4. - 6. Lebenswoche)

auffällig

unauffällig

wenn auffällig, was:

U4 (3. - 4. Lebensmonat)

auffällig口

unauffälliga

wenn auffällig, was:
U5 (6. - 7. Lebensmonat)
auffällig口
unauffällig

wenn auffällig, was:

U6 (10. - 12. Lebensmonat)

auffällig口

unauffällig

wenn auffällig, was.

U7 (21. - 24. Lebensmonat)

auffällig口

unauffällig

wenn auffällig, was:

\section{Fragen zur Mutter}

Schulabschluß:

Aktuell berufstätig: nein $\square$

ja $\square$

Wenn ja, in welchem Umfang:

ganztags $\square$

dreivierteltags $\square \quad$ halbtags $\square$

weniger als halbtags $\square$

\section{Fragen zum Vater}

Schulabschluß:

Aktuell berufstätig: nein $\square$ ja $\square$ Wenn ja, in welchem Umfang:

ganztags $\square \quad$ dreivierteltags $\square$ halbtags $\square$ weniger als halbtags $\square$

\section{Fragen zum Wohnort des Kindes}

Wohnort: Stadt (>10.000 Einwohner) a L

\section{Welches sind die täglichen Bezugspersonen des Kindes?}

Bitte numerieren Sie die Personen ab 1 aufwärts, wer die meiste Zeit des Tages mit dem Kind verbringt

( meiste Zeit $=1$, nächstmeiste Zeit =2, usw.; Beispiel: Mutter =1, Opa =2, Tagesmutter =3):

Mutter

Vater

Lebensgefährte

Lebensgefährtin

Geschwister

Oma

Opa
Tagesmutter

NachbarIn

Au-pair Mädchen

ErzieherIn (institutionell)

Tante

Onkel 
Im folgenden finden Sie eine Wortliste. Bitte kreuzen Sie alle Wörter an, die Ihr Kind als solche spricht. Dabei kommt es nicht auf die absolut korrekte Aussprache an, aber andere Bezeichnungen als die vorgegebenen gelten nicht.

Bitte machen Sie bei jedem Wort ein Kreuz in das ja- oder nein- Kästchen bzw. ergänzen nicht aufgeführte Wörter.

\begin{tabular}{|c|c|c|c|}
\hline$\downarrow$ Körperteile & $\begin{array}{c}\downarrow \text { Fahrzeuge/Ausstattung } \\
\text { Zubehör }\end{array}$ & $\downarrow$ Draußen & \\
\hline jad neind & jad neind & Baum & jab nein $\square$ \\
\hline ja $\square$ nein $\square$ & jaD nein $\square$ & Blatt & jaD nein $\square$ \\
\hline jad neina & jaD nein $\square$ & Blume & jaD neina \\
\hline jad neina & jaD nein & Garten & jal nein $\square$ \\
\hline jad neina & jad neina & Gras & ja $\square$ nein $\square$ \\
\hline jaD neina & Eisenbahn jaD neind & Mond & jab nein \\
\hline jad neind & ja $\square$ nein $\square$ & Sand & jaD nein $\square$ \\
\hline jad neina & ja $\square$ nein $\square$ & Sonne & jab nein $\square$ \\
\hline jad neina & ja $\square$ nein $\square$ & Stein & ja $\square$ nein $\square$ \\
\hline jad neina & Motorrad jal nein & Straße & jab nein $\square$ \\
\hline jad neina & jaD nein $\square$ & Tor & jab nein $\square$ \\
\hline jad neind & jaD nein & Wolke & jal nein $\square$ \\
\hline jad neina & jaD nein $\square$ & & \\
\hline jad neina & & & \\
\hline jad neina & & & \\
\hline $\begin{array}{c}\downarrow \text { Spielzeuge / Spielplatz } \\
\text { Symbolfiguren }\end{array}$ & $\downarrow$ Tiere / Teile vom Tier & $\downarrow$ Kleidung / & Requisiten \\
\hline jad neina & jad neina & Body & jab neina \\
\hline jad neina & jad neina & Brille & jab nein $\square$ \\
\hline jad neina & jad neind & Hose & jal nein $\square$ \\
\hline jad neina & jad neina & Hut & jag nein $\square$ \\
\hline jad neind & jad neind & Jacke & jaD nein $\square$ \\
\hline jad neina & jad neina & Kette & jab nein $\square$ \\
\hline jad neina & jad neind & Kleid & jaD neina \\
\hline jad neina & jad neina & Mütze & jab nein $\square$ \\
\hline $\begin{array}{l}\begin{array}{l}\text { Sandkasten/- jaD neind } \\
\text { kiste }\end{array} \\
\end{array}$ & jaD neind & Pullover & jad neina \\
\hline jad neina & jad neina & Rucksack & jab nein $\square$ \\
\hline jad neind & jad neind & Schuh & jag nein $\square$ \\
\hline jad neind & jad neind & Schuhe & ja $\square$ nein $\square$ \\
\hline jad neina & jad neind & Stiefel & jab nein \\
\hline & jad neina & Strümpfe & jal nein $\square$ \\
\hline & jaD nein $\square$ & T-Shirt & jab neina \\
\hline & jaD nein $\square$ & Tuch & jal neina \\
\hline & Schnecke jad neind & Unterhose & ja口 nein \\
\hline & jaD nein $\square$ & Windel & jab nein $\square$ \\
\hline & jaD nein & & \\
\hline & jaD nein $\square$ & & \\
\hline
\end{tabular}




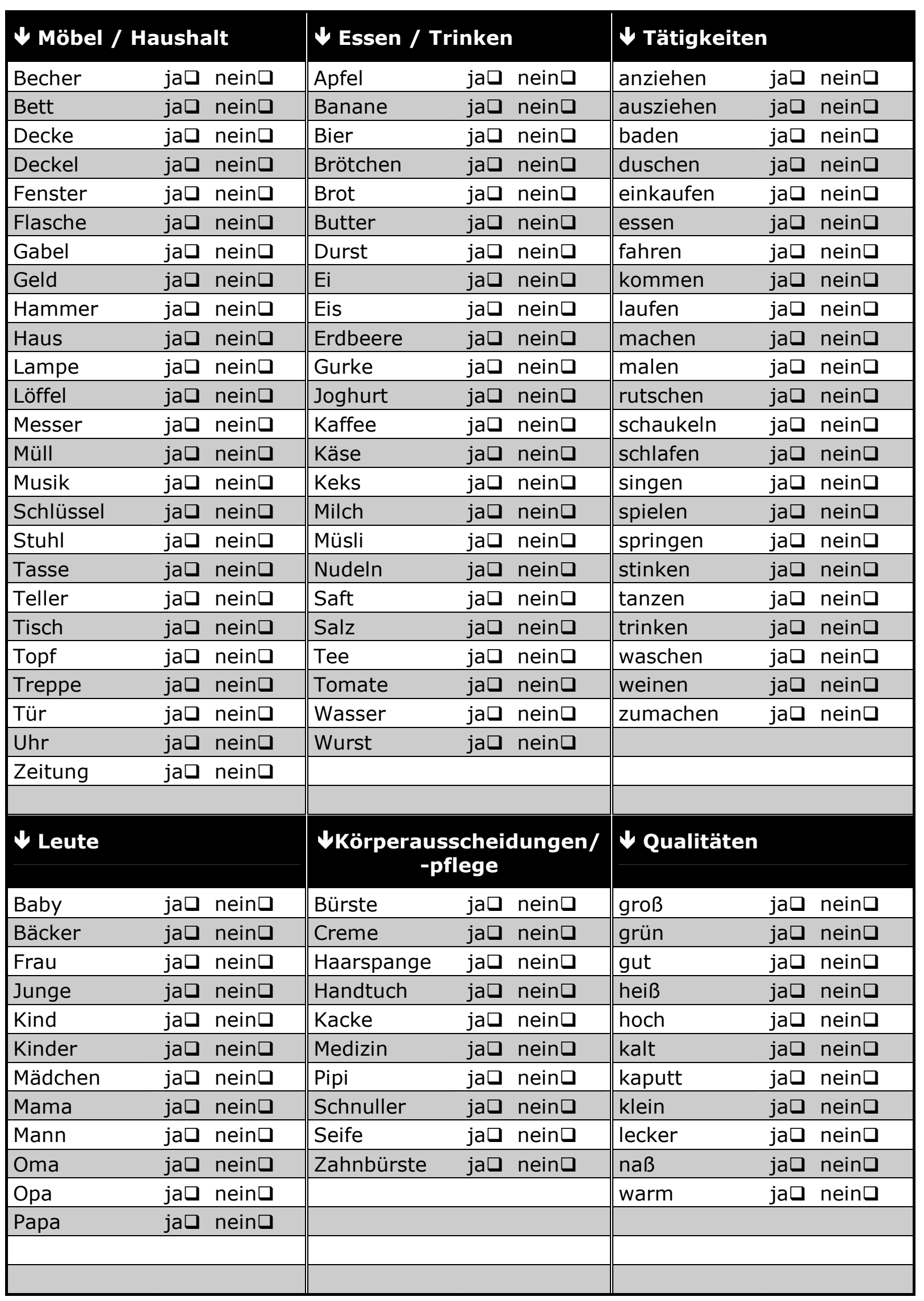




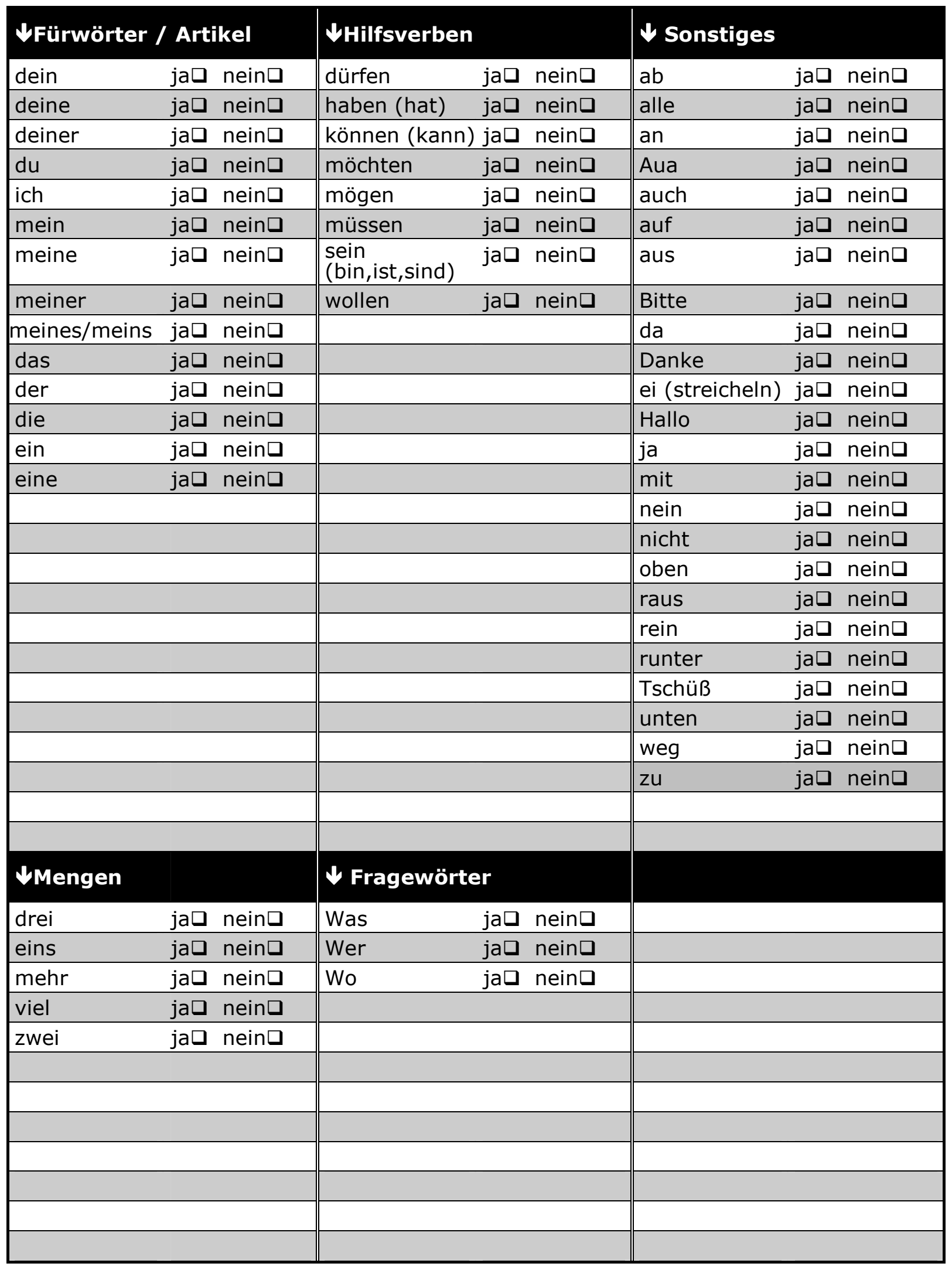

Bitte überprüfen Sie, ob Sie auch kein Wort ausgelassen haben! 
Weitere Wörter, die ihr Kind spricht:

Kombiniert Ihr Kind zwei Wörter (wie "mehr Keks", "Papa da") ? Nennen Sie ein paar Beispiele:

Gebraucht Ihr Kind überwiegend:

$$
\begin{aligned}
& \text { Ein-Wort-Äußerungen } \\
& \text { Zwei-Wort-Äußerungen } \\
& \text { Mehr als Zwei-Wort-Äußerungen }
\end{aligned}
$$

Bitte schreiben Sie abschließend drei typische Beispiele für Äußerungen Ihres Kindes auf:

Der Elternfragebogen wurde ausgefüllt von:

Vater $\square$

\section{Herzlichen Dank für Ihre Mitarbeit.}


Anhang B

Anamnesebogen $t_{1}$

\section{Anamnesebogen ( $\begin{aligned} & \text { 1 1) } \\ & \text { A }\end{aligned}$}

Name:

Vorname:

Geschlecht:

\section{Geburtsdatum:}

Datum der Untersuchung:

Alter des Kindes:

Adresse:

\section{Behandelnder Kinderarzt:}

Fragen zum Kind

Wie viele Geschwister Ihres Kindes leben in der Familie?

Das wievielte Kind ist Ihr Sohn/Tochter?

1. !

2. !

3. !

4. !

5. !

6. !

Zwilling: nein! ja !

Hat ihr Kind seit der letzten Untersuchung Mittelohrentzündungen gehabt? nein ! ja !

Wenn ja, wie viele:

Ist Ihr Kind in einer Krabbelgruppe, Spielkreis, Kindergarten,o.ä.?

nein !

ja !

Ca. wie viele Stunden in der Woche ist Ihr Kind dort insgesamt?
bis 4 Stunden
5 bis 10 Stunden
11 bis 20 Stunden
mehr als 20 Stunden !

Seit wann ist Ihr Kind dort?

Datum:

Gab es in den Vorsorgeuntersuchungen seit letztem Jahr Auffälligkeiten? Wenn ja, welche?

Sonstige Erkrankungen, Krankenhausaufenthalte, Operationen (Monat/Jahr des Auftretens und Dauer in Wochen):

Regelmäßige Medikamente:

Welche Auffälligkeiten haben Sie in der letzten Zeit festgestellt? 
Behandlungen (z.B. Ergotherapie, Krankengymnastik, Logopädie):

Gibt es Sprachprobleme/-störungen innerhalb der Familie (z.B. Onkel)?

nein ! ja ! Wenn ja, welche?

\section{Fragen zur Mutter}

Aktuell berufstätig: nein ! ja ! Wenn ja, in welchem Umfang:

ganztags! dreivierteltags! halbtags! weniger als halbtags!

\section{Fragen zum Vater}

Aktuell berufstätig:

ganztags !

ja!

Wenn ja, in welchem Umfang:

halbtags ! weniger als halbtags !

\section{Fragen zum Wohnort des Kindes}

Wohnort: Stadt (>10.000 Einwohner)!

Land ( $<10.000$ Einwohner) !

\section{Welches sind die täglichen Bezugspersonen des Kindes?}

Bitte numerieren Sie die Personen ab 1 aufwärts, wer die meiste Zeit des Tages mit dem

Kind verbringt

(meiste Zeit $=1$, nächstmeiste Zeit $=2$, usw.; Beispiel: Mutter $=1$, Oma $=2$, Nachbar $=3$ )

Mutter

Tagesmutter

Vater

NachbarIn

Lebensgefährte

Au-pair Mädchen

Lebensgefährtin

ErzieherIn

Geschwister

Tante

Oma

Onkel

Opa 
Anhang C

Erweiterung des Anamnesebogens zu $t_{2}$

Bitte beurteilen Sie jede Aussage. Machen Sie jeweils ein Kreuz entsprechend des Grades Ihrer Zustimmung bzw. Ablehnung. Vielen Dank

\section{Fragen zum Kind:}

1. Unser Kind kann sich gut alleine beschäftigen.

\begin{tabular}{|ccccc|}
\hline 0 & 1 & 2 & 3 & 4 \\
trifft gar nicht zu & kaum & weniger & überwiegend & trifft völlig zu \\
\hline
\end{tabular}

2. Unser Kind kann sich ausdauernd mit einer Sache beschäftigen.

\begin{tabular}{|ccccc|}
\hline 0 & 1 & 2 & 3 & 4 \\
trifft gar nicht zu & kaum & weniger & überwiegend & trifft völlig zu \\
\hline
\end{tabular}

3. Unser Kind kann gut Regeln einhalten.

\begin{tabular}{|ccccc|}
\hline 0 & 1 & 2 & 3 & 4 \\
trifft gar nicht zu & kaum & weniger & überwiegend & trifft völlig zu \\
\hline
\end{tabular}

4. Unser Kind hat Spaß am Sprechen (Sprechfreude).

\begin{tabular}{|ccccc|}
\hline 0 & 1 & 2 & 3 & 4 \\
trifft gar nicht zu & kaum & weniger & überwiegend & trifft völlig zu \\
\hline
\end{tabular}

5. Unser Kind spielt selbständig mit Sprache (denkt sich z.B. Phantasiewörter aus oder erfindet Reime).

\begin{tabular}{|ccccc|}
\hline 0 & 1 & 2 & 3 & 4 \\
trifft gar nicht zu & kaum & weniger & überwiegend & trifft völlig zu \\
\hline
\end{tabular}

6. Unser Kind spielt überwiegend Bewegungsspiele (fangen, klettern, etc.).

\begin{tabular}{|ccccc|}
\hline 0 & 1 & 2 & 3 & 4 \\
trifft gar nicht zu & kaum & weniger & überwiegend & trifft völlig zu \\
\hline
\end{tabular}

7. Unser Kind spricht in kurzen Sätzen.

\begin{tabular}{|ccccc|}
\hline 0 & 1 & 2 & 3 & 4 \\
trifft gar nicht zu & kaum & weniger & überwiegend & trifft völlig zu \\
\hline
\end{tabular}


8. Der aktive Wortschatz unseres Kindes (die Vielzahl der Wörter, die unser Kind spricht) ist groß im Vergleich zu anderen Kindern seines Alters.

\begin{tabular}{|ccccc|}
\hline 0 & 1 & 2 & 3 & 4 \\
trifft gar nicht zu & kaum & weniger & überwiegend & trifft völlig zu \\
\hline
\end{tabular}

9. Unser Kind kann sicher die Mehrzahl von Wörtern bilden (z.B. VogelVögel, Gabel-Gabeln).

\begin{tabular}{|ccccc|}
\hline 0 & 1 & 2 & 3 & 4 \\
trifft gar nicht zu & kaum & weniger & überwiegend & trifft völlig zu \\
\hline
\end{tabular}

10. Unser Kind versteht längere Aufforderungen (z.B. "Gib mir die Kiste, nachdem du einen Knopf reingelegt hast").

\begin{tabular}{|ccccc|}
\hline 0 & 1 & 2 & 3 & 4 \\
trifft gar nicht zu & kaum & weniger & überwiegend & trifft völlig zu \\
\hline
\end{tabular}

11. Unserem Kind gelingt das Nachsprechen unbekannter Wörter (wie z.B. "Ronterklabe").

\begin{tabular}{|ccccc|}
\hline 0 & 1 & 2 & 3 & 4 \\
trifft gar nicht zu & kaum & weniger & überwiegend & trifft völlig zu \\
\hline
\end{tabular}

12. Unserem Kind gelingt das Nachsprechen von längeren Sätzen (wie z.B. "Lena lacht, nachdem sie gekitzelt wurde").

\begin{tabular}{|ccccc|}
\hline 0 & 1 & 2 & 3 & 4 \\
trifft gar nicht zu & kaum & weniger & überwiegend & trifft völlig zu \\
\hline
\end{tabular}

13. Unser Kind kann sicher entscheiden, ob sich zwei Wörter ähnlich anhören (reimen) (z.B. Buch-Tuch, Kind-Wind).

\begin{tabular}{|ccccc|}
\hline 0 & 1 & 2 & 3 & 4 \\
trifft gar nicht zu & kaum & weniger & überwiegend & trifft völlig zu \\
\hline
\end{tabular}

14. Unser Kind erkennt sicher, ob ein einzelner Laut in einem Wort vorkommt (z.B. "Hörst du au in Auto?", "Hörst du e in Uniform?").

\begin{tabular}{|ccccc|}
\hline 0 & 1 & 2 & 3 & 4 \\
trifft gar nicht zu & kaum & weniger & überwiegend & trifft völlig zu \\
\hline
\end{tabular}

15. Unser Kind kann Wörter sicher in ihre einzelnen Silben zerlegen (z.B. Fe-der-ball sagen und dazu klatschen).

\begin{tabular}{|ccccc|}
\hline 0 & 1 & 2 & 3 & 4 \\
trifft gar nicht zu & kaum & weniger & überwiegend & trifft völlig zu \\
\hline
\end{tabular}


16. Unser Kind macht sich Gedanken darüber, was andere Menschen denken, glauben und wissen.

\begin{tabular}{|ccccc|}
\hline 0 & 1 & 2 & 3 & 4 \\
trifft gar nicht zu & kaum & weniger & überwiegend & trifft völlig zu \\
\hline
\end{tabular}

\section{Fragen zum familiären Alltag:}

17. In unserer Familie werden Reimspiele gespielt.

\begin{tabular}{|ccccc|}
\hline 0 & 1 & 2 & 3 & 4 \\
trifft gar nicht zu & kaum & weniger & überwiegend & trifft völlig zu \\
\hline
\end{tabular}

18. In unserer Familie wird mit Sprache gespielt (z.B. Phantasiewörter erfunden).

\begin{tabular}{|ccccc|}
\hline 0 & 1 & 2 & 3 & 4 \\
trifft gar nicht zu & kaum & weniger & überwiegend & trifft völlig zu \\
\hline
\end{tabular}

19. In unserer Familie wird häufig gesungen.

\begin{tabular}{|ccccc|}
\hline 0 & 1 & 2 & 3 & 4 \\
trifft gar nicht zu & kaum & weniger & überwiegend & trifft völlig zu \\
\hline
\end{tabular}

20. In unserer Familie werden regelmäßig Bilderbücher angeschaut.

\begin{tabular}{|ccccc|}
\hline 0 & 1 & 2 & 3 & 4 \\
trifft gar nicht zu & kaum & weniger & überwiegend & trifft völlig zu \\
\hline
\end{tabular}

21. In unserer Familie gibt es einen strukturierten Tagesablauf.

\begin{tabular}{|ccccc|}
\hline 0 & 1 & 2 & 3 & 4 \\
trifft gar nicht zu & kaum & weniger & überwiegend & trifft völlig zu \\
\hline
\end{tabular}

22. In unserer Familie gibt es feste Regeln.

\begin{tabular}{|ccccc|}
\hline 0 & 1 & 2 & 3 & 4 \\
trifft gar nicht zu & kaum & weniger & überwiegend & trifft völlig zu \\
\hline
\end{tabular}


23. In unserer Familie wird regelmäßig vorgelesen.

\begin{tabular}{|ccccc|}
\hline 0 & 1 & 2 & 3 & 4 \\
trifft gar nicht zu & kaum & weniger & überwiegend & trifft völlig zu \\
\hline
\end{tabular}

24. In unserer Familie werden Sprechfehler der Kinder konsequent korrigiert.

\begin{tabular}{|ccccc|}
\hline 0 & 1 & 2 & 3 & 4 \\
trifft gar nicht zu & kaum & weniger & überwiegend & trifft völlig zu \\
\hline
\end{tabular}


Anhang D

\section{Elternfragebogen $t_{3}$}

Abteilung Pädagogische Psychologie und Entwicklungspsychologie, Universität Göttingen

A. Klonk (Dipl.-Psych.)

E. Schlösser

\section{Elternfragebogen (T3)}

\section{Name des Kindes:}

\section{Geburtsdatum:}

\section{Datum der Untersuchung:}

\section{Fragen zum Kind}

Wie viele Geschwister Ihres Kindes leben in der Familie?

Das wievielte Kind ist Ihre Tochter / Ihr Sohn?

Gab es in den letzten 2 Jahren Veränderungen im häuslichen Umfeld Ihres Kindes (z.B. Geburten, Sterbefälle, Umzug, Berufstätigkeit der Eltern,...)?
nein
ja (bitte beschreiben)

Hat Ihr Kind seit der letzten Untersuchung Mittelohrentzündungen gehabt?

$\square$ nein

$\square$ ja, und zwar mal

Sonstige Erkrankungen / Behandlungen der Ohren (Paukenerguss, Paukenröhrchen, auffälliger Hörtest, übermäßig viel Ohrenschmalz, ...):

Gab es in den letzten 2 Jahren bei Untersuchungen durch den Kinderarzt Auffälligkeiten? 
Sonstige Erkrankungen / Krankenhausaufenthalte / Unfälle / Operationen (Monat / Jahr des Auftretens und Dauer in Wochen):

Regelmäßige Medikamente:

Welche Auffälligkeiten haben Sie in der letzten Zeit beobachtet (Verhalten, Befinden)?

Ist oder war Ihr Kind in regelmäßiger Behandlung (Ergotherapie, Krankengymnastik, Logopädie, LRS-Therapie, Dyskalkulietherapie)?

Seit wann geht Ihr Kind in die Schule? derzeitige Klasse:

Wie viel Zeit verbringt es durchschnittlich nachmittags mit Hausaufgaben? $\min$ Gibt es in der Schule Schwierigkeiten?
$\square$ nein
$\square$ ja (bitte beschreiben)

Gibt es in der Familie Personen mit Lese- oder Rechtschreibschwierigkeiten?

$\square$ nein $\quad \square$ ja: wer?

Art der Schwierigkeiten:

Wie viel Zeit verbringt Ihr Kind durchschnittlich täglich mit Lesen (ohne Hausaufgaben)? $\min$

Lesen Sie Ihrem Kind vor? Wenn ja: Wie lange durchschnittlich täglich? $\min$

Wie viel Zeit verbringt Ihr Kind durchschnittlich täglich mit Fernsehen? werktags: min am Wochenende: $\min$

Wie viel Zeit verbringt Ihr Kind durchschnittlich täglich am Computer? werktags: min am Wochenende: $\min$ 